\title{
ESTIMATION OF MELTING POINTS OF ORGANIC COMPOUNDS
}

\author{
By \\ Akash Jain \\ Copyright (C) Akash Jain 2005 \\ A Dissertation Submitted to the Faculty of the \\ DEPARTMENT OF PHARMACEUTICAL SCIENCES \\ In Partial Fulfillment of the Requirements \\ For the Degree of \\ DOCTORAL OF PHILOSOPHY \\ In the Graduate College \\ THE UNIVERSITY OF ARIZONA
}




\section{THE UNIVERSITY OF ARIZONA GRADUATE COLLEGE}

As members of the Dissertation Committee, we certify that we have read the dissertation prepared by Akash Jain entitled Estimation of Melting Points of Organic Compounds and recommend that it be accepted as fulfilling the dissertation requirement for the Degree of Doctor of Philosophy

\section{Samuel H. Yalkowsky, Ph.D.}

Date: $(10-17-05)$

Date: $\underline{(10-17-05)}$

Michael Mayersohn, Ph.D.

Date: $(10-17-05)$

Paul B. Myrdal, Ph.D.

Date: $\underline{(10-17-05)}$

\section{Srini Raghavan, Ph.D.}

Date: (

Final approval and acceptance of this dissertation is contingent upon the candidate's submission of the final copies of the dissertation to the Graduate College.

I hereby certify that I have read this dissertation prepared under my direction and recommend that it be accepted as fulfilling the dissertation requirement.

Date: $\underline{(10-17-05)}$

\section{Dissertation Director: Samuel H. Yalkowsky, Ph.D.}




\section{STATEMENT BY AUTHOR}

This dissertation has been submitted in partial fulfillment of requirements for an advanced degree at the University of Arizona and is deposited at the University Library to be made available to borrowers under rules of the Library.

Brief quotations from this dissertation are allowable without special permission, provided that accurate acknowledgement of the source is made. Requests for permission for extended quotation from or reproduction of this manuscript in whole or in part may be granted by the copyright holder.

AKASH JAIN 


\section{ACKNOWLEDGEMENTS}

I would like to express my gratitude to my advisor, Dr Samuel Yalkowsky, for providing me the opportunity and financial support to earn my doctoral degree in Pharmaceutical Sciences. I sincerely thank him for his continued guidance, encouragement and mentorship over the years I spent at the University of Arizona. I will cherish his scientific values, virtues and philosophies for life.

I would like to thank my major committee members, Dr Michael Mayersohn and Dr Paul Myrdal, for their guidance and comments in not only preparing this dissertation but for my entire period of education at the University of Arizona. I would also like to express my gratitude to my minor committee members, Dr Srini Ragahvan and Dr Neal Armstrong, for dedicating their time to serve on my committee.

I would like to thank my past and present colleagues at the University of Arizona for being there for me whenever I needed them. I will inherit all the good times we have had working together and I am pleased to have made some friends for life.

I would like to thank my professors back in India, especially Dr T.R.Saini and Dr. S.C. Chaturvedi, for encouraging me to pursue a doctoral degree in Pharmaceutical Sciences.

At last, I would like to thank my family: my parents and sister for their love and inspiration, my fiancée Bhumika for all her love and patience and her family for their care and support. 


\section{DEDICATION}

To my parents and Bhumika 


\section{TABLE OF CONTENTS}

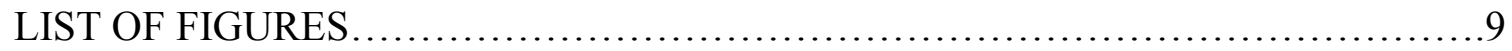

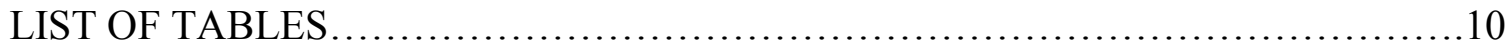

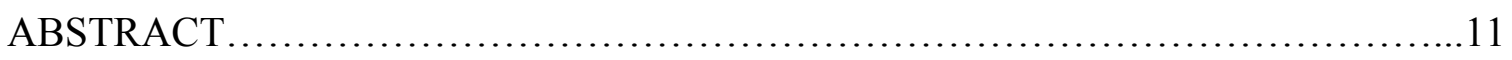

CHAPTER 1: INTRODUCTION........................................... 12

CHAPTER 2: ENTHALPY OF MELTING...................................... 19

INTRODUCTION .................................................... 19

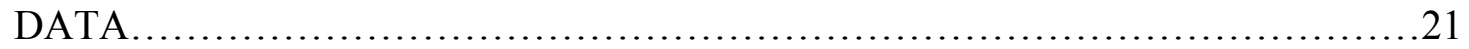

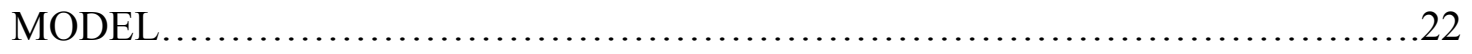

Group Definition.....................................................22

Group Environment....................................................23

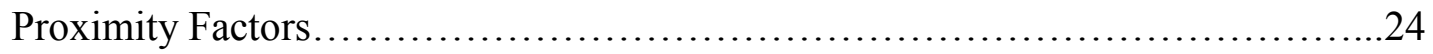

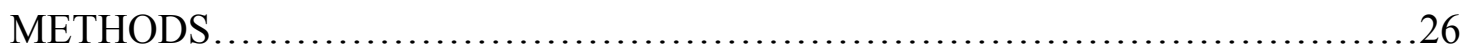

RESULTS AND DISCUSSION ......................................... 26

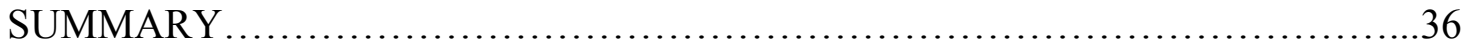

CHAPTER 3: ENTROPY OF MELTING ........................................ 38

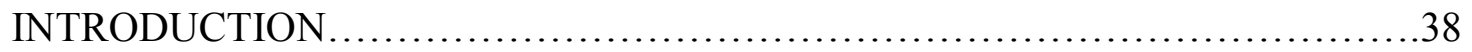

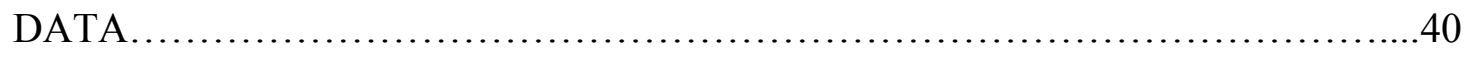

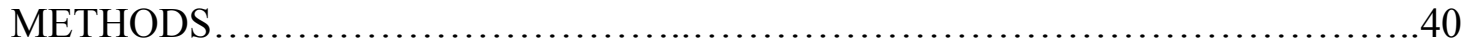

Molecular Rotational Symmetry Number..................................40

Molecular Flexibility Number.........................................42 
TABLE OF CONTENTS - Continued

RESULTS AND DISCUSSION ........................................44

SUMMARY ............................................................... 48

CHAPTER 4: MELTING POINTS ..........................................49

INTRODUCTION ......................................................49

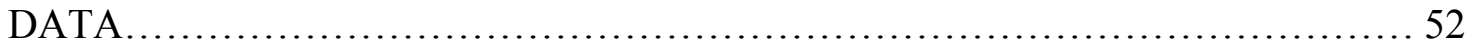

METHODS.............................................................. 52

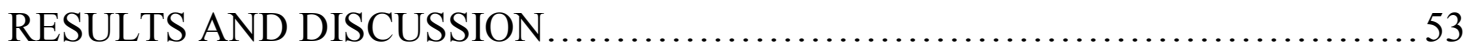

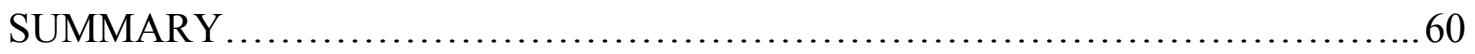

CHAPTER 5: COMPARISON OF TWO METHODS FOR PREDICTING MELTING

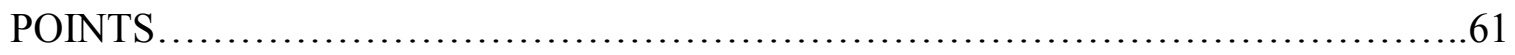

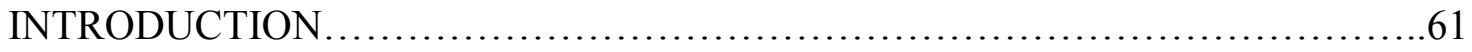

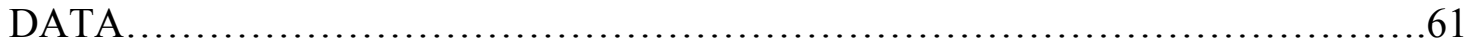

METHODS..............................................................61

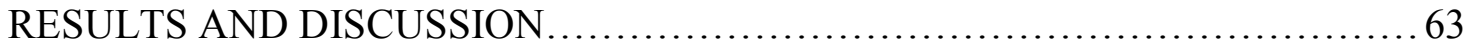

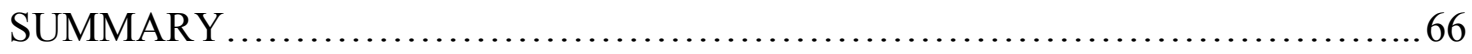

APPENDIX A. Examples of predicting enthalpy of melting $\left(\Delta \mathrm{H}_{\mathrm{m}}\right)$, entropy of melting $\left(\Delta \mathrm{S}_{\mathrm{m}}\right)$ and melting point $\left(\mathrm{T}_{\mathrm{m}}\right)$ using the developed model........................67 APPENDIX B. Experimental and predicted enthalpy of melting $\left(\Delta \mathrm{H}_{\mathrm{m}}\right)$ for 1663 organic

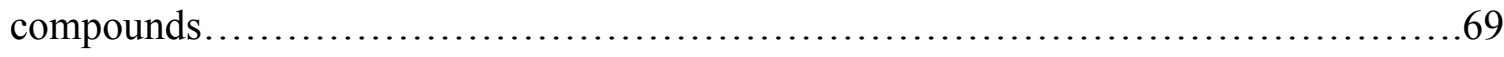




\section{TABLE OF CONTENTS - Continued}

APPENDIX C. Rotational symmetry number $(\sigma)$, Flexibility count $(\Phi)$, experimental entropy of melting and predicted entropy of melting $\left(\Delta \mathrm{S}_{\mathrm{m}}\right)$ for 1663 organic

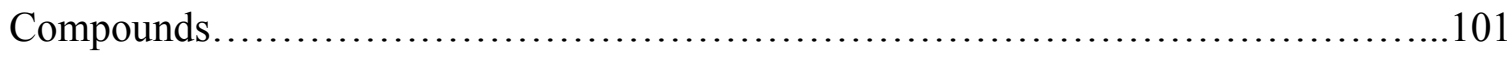

APPENDIX D. Experimental and predicted Melting Points $\left(T_{m}\right)$ for 2230 organic

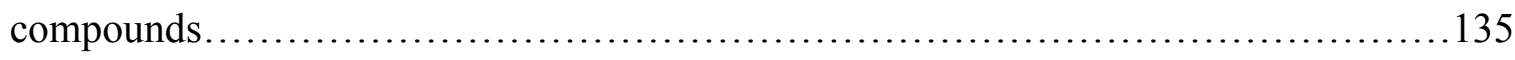

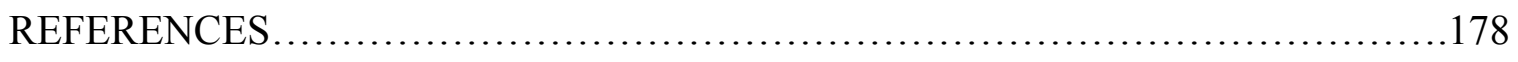




\section{LIST OF FIGURES}

Figure 1.1: UPPER (Unified Physical Property Estimation Relationships) Scheme...... .12

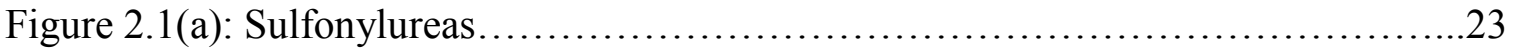

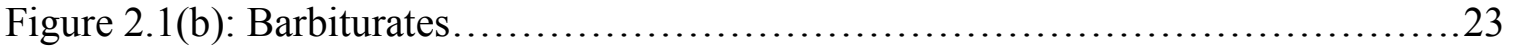

Figure2.2: Molecular environment descriptors in the fragmentation scheme............24

Figure 2.3: Intramolecular hydrogen bonding patterns ............................... 25

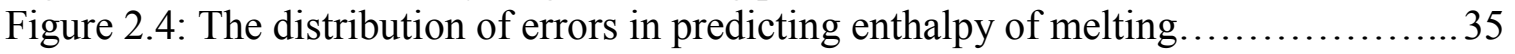

Figure 3.1: Symmetry numbers for several molecules........................... 42

Figure 3.2: The distribution of errors in predicting entropy of melting .................45

Figure 3.3: Error distribution as a function of the logarithm of the rotational

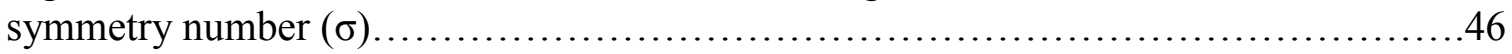

Figure 3.4: Error distribution as a function of the logarithm of the molecular

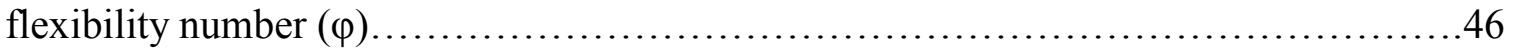

Figure 4.1: Observed versus predicted melting points for 2230 organic compounds.....53

Figure 4.2: Error distribution in melting point prediction using the proposed model......54

Figure 4.3: Predicted versus observed melting points for 152 drugs

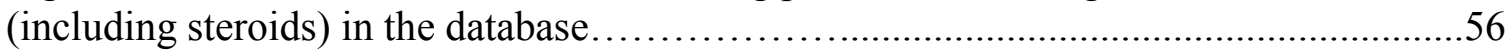

Figure 4.4: Predicted versus observed melting points for 149 environmental

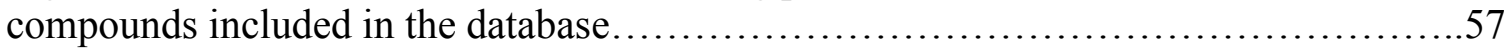

Figure 4.5: Proximity factors for intramolecular hydrogen bonding and geminal

fluorine atoms in Dinitramine................................................ 58

Figure 4.6: Experimental melting points of n-alkanes (C2-C190) ..................59

Figure 5.1: Observed versus predicted melting points (using UPPER)

for 2230 compounds

Figure 5.2: Observed versus predicted melting points (using MPBPWIN) for 2230 compounds

Figure 5.3: AAE in melting point prediction of symmetrical molecules using

the two models

Figure 5.4: AAE in melting point predictions of flexible molecules using

the two models 


\section{LIST OF TABLES}

Table 1.1: Experimental melting points $\left({ }^{\circ} \mathrm{C}\right)$ of some homologous series................14

Table 1.2: Experimental melting points for some octanes........................... 15

Table 1.3: Experimental melting points for isomeric arenes........................ 15

Table 1.4: Experimental melting points $\left({ }^{\circ} \mathrm{C}\right)$ for some disubstituted benzenes............15

Table 2.1: A list of molecular fragments and proximity factors along with their enthalpy contribution values................................................. 27

Table 2.2: Frequency distribution of the average absolute errors in enthalpy of

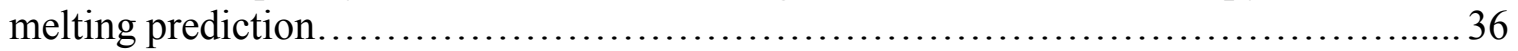

Table 3.1: Examples of some molecular flexibility numbers.......................44

Table 3.2: Average absolute errors in entropy of melting prediction at different

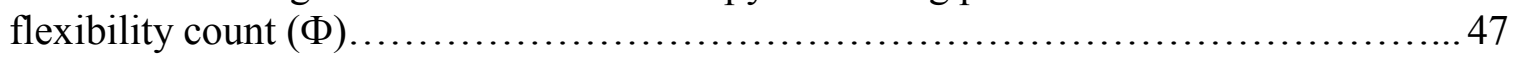

Table 3.3: Frequency distribution of the average absolute errors in entropy of melting prediction......................................................... 47

Table 4.1: Average Absolute Errors in general melting point estimation methods........51

Table 4.2: Frequency distribution of the average absolute errors in melting point prediction............................................................... 54

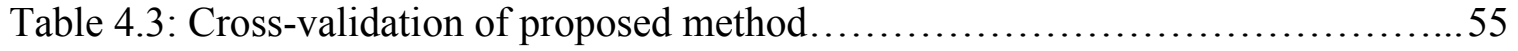




\begin{abstract}
Melting point finds applications in chemical identification, purification and in the calculation of a number of other physicochemical properties such as vapor pressure and aqueous solubility. Despite the availability of enormous amounts of experimental data, no generally applicable methods have been developed to estimate the melting point of a compound from its chemical structure. A quick estimation of melting point can be a useful tool in the design of new chemical entities.

In this dissertation, a simple means of estimating the melting points for a large variety of pharmaceutically and environmentally relevant organic compounds is developed. Melting points are predicted from the separate calculation of the enthalpy and entropy of melting directly from the chemical structure. The entropy of melting is calculated using a semiempirical equation based on only two non-additive molecular parameters. This equation is validated and refined using a large collection of experimental entropy of melting values. The enthalpy of melting is calculated by additive group contributions.

Melting points are estimated from the ratio of the enthalpy of melting and the entropy of melting. All of the methods and group contributions developed in this study are compatible with the UPPER (Unified Physical Property Estimating Relationships) scheme. The predicted melting points are compared to experimental melting points for over 2200 organic compounds collected from the literature. The average absolute error in melting point prediction is $30.1^{\circ}$. This is a very reasonable estimate considering the size and diversity of the dataset used in this study.
\end{abstract}




\section{CHAPTER 1: INTRODUCTION}

Melting point is of great importance in the calculation of several physicochemical properties. As seen in figure 1.1, melting point $\left(\mathrm{T}_{\mathrm{m}}\right)$ is an integral part of Yalkowsky's UPPER scheme (Yalkowsky et al., 1994).

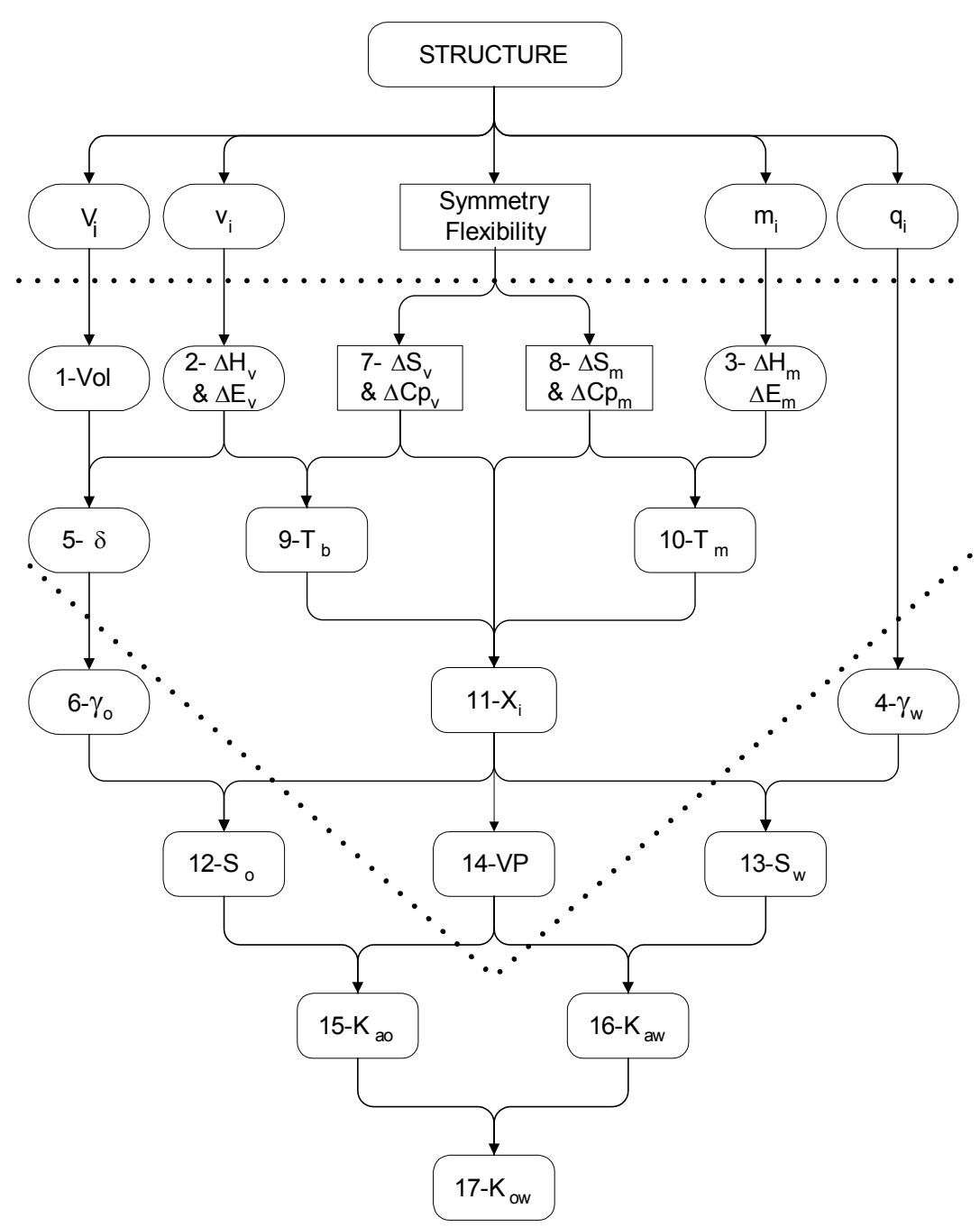

Figure 1.1. UPPER (Unified Physical Property Estimation Relationships) Scheme 
$T_{m}$ is used in the estimation of ideal crystalline solubility $\left(\mathrm{X}_{\mathrm{i}}\right)$ which in turn is used to calculate octanol solubility $\left(\mathrm{S}_{\mathrm{o}}\right)$, vapor pressure $(\mathrm{VP})$ and aqueous solubility $\left(\mathrm{S}_{\mathrm{w}}\right) . \mathrm{T}_{\mathrm{m}}$ is used as a descriptor for properties like aqueous solubility (Yalkowsky, 1981, Jain et al., 2001, Ran et al., 2001), vapor pressure (Katritzky et al., 2001), liquid viscosity (Nikmo et al., 2002) and behavior of eutectic compositions (Law et al., 2002).

Melting point is useful in pre-formulation studies for characterization and screening of drugs. Melting point has great significance in the field of environmental science as well. It indicates the physical state of a chemical at ambient temperatures and provides valuable information regarding its handling and treatment. Because of its relationship with vapor pressure and aqueous solubility, melting point is an important property for understanding the absorption, distribution and transport of chemicals in the environment (Dearden, 2003) as well as in the human body.

Unfortunately, there are few models available for predicting melting point, and compounds have to be synthesized and their melting points experimentally measured before any other properties are predicted. Often there are problems of thermal instability involved in the measurement. Although a large number of experimental melting points are available, often there are chemicals released into the environment which have no reported melting points. In addition, there are always new chemical entities submitted to regulatory agencies without any experimental data (Dearden, 2003). In an attempt to minimize the above mentioned problems, the development of a simple, accurate and widely applicable prediction model for melting points of organic compounds is desired. 
Although melting point is the most widely reported property for organic compounds, its relationship to chemical structure is not clearly understood. Despite the availability of vast amounts of experimental melting point data, there is no general relationship relating melting point directly to chemical structure. Melting points, although analogous to boiling points, are more difficult to predict. Boiling points generally show a regular increase within homologous series and are nearly identical for isomers. However, the experimental data in Table 1.1 suggest that melting points do not show a regular increase with homologation. Also the melting points of alkyl isomers (Table 1.2), isomeric arenes (Table 1.3) and disubstituted benzenes (Table 1.4) are widely different and irregular. As a result, no group contribution schemes are capable of adequately predicting melting points, even for simple molecules.

Table 1.1. Experimental melting points $\left({ }^{\circ} \mathrm{C}\right)$ of some homologous series

\begin{tabular}{lccc} 
Alkyl Chain length & Alkanes & Alkanols & Alkanoic Acids \\
\hline 1 & -183 & -98 & 8 \\
2 & -184 & -114 & 26 \\
3 & -188 & -124 & -20 \\
4 & -138 & -88 & -8 \\
5 & -130 & -77 & -34 \\
6 & -95 & -47 & -3 \\
7 & -90 & -33 & -7 \\
8 & -57 & -15 & 17 \\
9 & -53 & -8 & 12 \\
10 & -30 & 6 & 31 \\
11 & -9 & 25 & 29 \\
12 & 6 & 40 & 54 \\
13 & 18 & 50 & 62 \\
14 & 28 & 61 & 69 \\
\hline
\end{tabular}


Table 1.2. Experimental melting points for some octanes

\begin{tabular}{lc} 
Compound & Melting Point $\left({ }^{\circ} \mathrm{C}\right)$ \\
\hline n-octane & -56 \\
2-methylheptane & -109 \\
3-methylheptane & -120 \\
4-methylheptane & -121 \\
2,2-dimethylhexane & -121 \\
2,5-dimethylhexane & -91 \\
3,3-dimethylhexane & -126 \\
2,2,3-trimethylpentane & -113 \\
2,2,4-trimethylpentane & -107 \\
2,3,3-trimethylpentane & -109 \\
2,3,4-trimethylpentane & -109 \\
2,2,3,3-tetramethylbutane & 101 \\
\hline
\end{tabular}

Table 1.3. Experimental melting points for isomeric arenes

\begin{tabular}{lc} 
Compound & Melting Point $\left({ }^{\circ} \mathrm{C}\right)$ \\
\hline o-xylene & -25 \\
m-xylene & -48 \\
p-xylene & 13 \\
hexylbenzene & -62 \\
p-diisopropylbenzene & -17 \\
s-triethylbenzene & -66 \\
hexamethylbenzene & 166 \\
anthracene & 216 \\
phenanthrene & 101 \\
1,2-benzanthracene & 162 \\
2,3-benzanthracene & 335 \\
3,4-benzphenanthrene & 168 \\
2,3-benzphenanthrene & 254 \\
9.10-benzphenanthrene & 199
\end{tabular}

Table 1.4. Experimental melting points $\left({ }^{\circ} \mathrm{C}\right)$ for some disubstituted benzenes

\begin{tabular}{lcrrrrr} 
Substituent & $\mathrm{CH} 3$ & $\mathrm{Cl}$ & $\mathrm{Br}$ & $\mathrm{I}$ & $\mathrm{NO}_{2}$ & $\mathrm{OCH}_{3}$ \\
\hline 1,2 -di- & -25 & -17 & -30 & -31 & 118 & -37 \\
1,3 -di- & -47 & -24 & 84 & 40 & 90 & 22 \\
$1,4-$ di- & 13 & 53 & 106 & 131 & 174 & 56 \\
\hline
\end{tabular}


A number of methods are currently available for estimating melting points. These methods are either based on group contributions (Joback et al., 1987, Simmamora et al., 1994, Krzyzaniak et al., 1995, Austin, 1930, Constantinou and Gani, 1994, Marrero and Gani, 2001) or physical and structural molecular parameters like molecular cohesiveness, bulkiness, hydrogen-bonding parameters and geometric factors (Kier et al., 1976, Dearden et al., 1988, Abramowitz et al., 1990). Although these methods are reasonably accurate, they are limited in their application as they are based on small datasets of simple molecules and/or homologous series. The group contribution schemes, as described earlier, cannot distinguish between isomers and are incapable of accounting for the geometric properties that influence the process of melting.

The goal of this dissertation is to develop a simple, accurate and widely applicable model for predicting melting points of organic compounds directly from chemical structure. The model is a combination of additive group contributions and non-additive molecular parameters which take into account the anomalies in experimental melting points of isomers and homologous series. In order to develop such a model, the following objectives are defined:

1. A reliable database of experimental values

2. A sound thermodynamic relationship with minimum number of assumptions

3. A reasonable molecular fragmentation pattern based upon a single molecular structure descriptor such as the SMILES string 
4. Ability to characterize molecular properties that are not strictly additive and constitutive, and to distinguish between isomers

5. Validation of the model on a wide range of simple structures

The UPPER-based estimation model is based on a fundamental thermodynamic relationship for phase transition. For a phase transition at equilibrium, the free energy change is equal to zero. The melting point is therefore related to the enthalpy $\left(\Delta \mathrm{H}_{\mathrm{m}}\right)$ and entropy of melting $\left(\Delta \mathrm{S}_{\mathrm{m}}\right)$ by the following relationship:

$$
\mathrm{T}_{\mathrm{m}}=\frac{\Delta \mathrm{H}_{\mathrm{m}}}{\Delta \mathrm{S}_{\mathrm{m}}}
$$

The calculations of each of these properties are described in the following chapters. Chapter 2 defines the enthalpy of melting. The enthalpy of melting is shown to be groupadditive. A novel molecular fragmentation scheme for calculating the enthalpy of melting is developed. The scheme is based on SMILES string notations. The wide application of the scheme is discussed as it applies to complex structures of drug-like molecules, herbicides and pesticides. Proximity factors included in the fragmentation scheme are discussed. A tabulation of the various molecular fragments and proximity factors present in the database is provided.

Chapter 3 deals with the entropy of melting. The semi-empirical equation of Dannenfelser et al. (1996, 1999) for entropy of melting is revisited and refined. The nonadditive molecular parameters, rotational symmetry and flexibility, that constitute the 
entropy of melting are discussed in detail. Methods for calculating each of these parameters are described.

Chapter 4 is based on the estimation of melting points from the combination of enthalpy and entropy of melting. The errors in melting point estimation are carefully evaluated. The prediction ability of the model is assessed using cross-validation.

In Chapter 5, the developed model is compared to an existing computer program that calculates melting points directly from SMILES string input. It is shown that our model provides more accurate estimations of melting points for over 2200 organic compounds. 


\section{CHAPTER 2: ENTHALPY OF MELTING}

\section{INTRODUCTION}

The heat required to melt one mole of a solid is the enthalpy of melting. It is the difference between the enthalpy of the solid and liquid phases. In the solid, as in the liquid, each molecular fragment contributes to dispersion, induction, dipolar, and hydrogen bonding interactions and the total intermolecular interaction energy is the sum of all the pair-wise group interactions. The enthalpy of melting can, therefore, be estimated by additive group contributions.

Group contributions are the simplest methods for estimating the enthalpy of melting. A number of group contribution methods are well documented in the literature (Joback et al. 1987, Chickos et al. 1989, Constantinou et al. 1994, Marrero et al. 2001). Joback et al. developed a first-order group contribution with 40 groups for organic compounds that may contain halogens, oxygen, nitrogen, and sulfur. The group contribution values were obtained from a small dataset of 155 compounds and the average absolute error in enthalpy of melting estimation was $2.03 \mathrm{~kJ} / \mathrm{mol}$. Chickos et al. (1989) estimated the enthalpies of melting for 191 hydrocarbons using a group contribution with 20 structural parameters. The average deviation for this method was $2.34 \mathrm{~kJ} / \mathrm{mol}$. Constantinou et al. developed a two-tier group contribution scheme containing 63 first-order groups and 40 second -order groups. While the first-order groups included the smaller, simpler groups, the second-order groups accounted for the proximity effects and to distinguish between 
the isomers. In order to include more complex structures in their scheme, Marrero and Gani (2001) developed a three-level group contribution scheme. The third level groups account for complex heterocyclic and large polyfunctional alicyclic compounds. Based on a dataset of 686 compounds, they obtained an average absolute error of $3.60 \mathrm{~kJ} / \mathrm{mol}$. Although the above mentioned methods have sufficient accuracy, they are either based on small, restricted datasets or consist of complex multi-level calculations that take more time and effort to use.

Another method for estimating the enthalpy of melting is from the product of the melting points and entropies of melting (Kirshenbaum, 1965, Chickos et al. 1999). This method was used mainly for estimating enthalpies of melting for homologous series and polymers. It is also useful for the generation of enthalpy data for compounds with no reported enthalpy values in the literature (Chickos et al. 2002).

In our model, the enthalpy of melting is estimated by a group contribution scheme. The scheme is developed from a large, structurally complex database of over 2200 organic compounds. Mathematically, the scheme can be written as

$$
\Delta \mathrm{H}_{\mathrm{m}}=\sum \mathrm{n}_{\mathrm{i}} \mathrm{m}_{\mathrm{i}}+\sum \mathrm{n}_{\mathrm{j}} \mathrm{m}_{\mathrm{j}}
$$

Where

$\mathrm{n}_{\mathrm{i}}=$ number of times that group $i$ appears in a compound $\mathrm{n}_{\mathrm{j}}=$ number of times that proximity factor $j$ appears in a compound 
$\mathrm{m}_{\mathrm{i}}=$ contribution of group $i$ to the enthalpy of melting $(\mathrm{kJ} / \mathrm{mol})$

$\mathrm{m}_{\mathrm{j}}=$ contribution of proximity factor $j$ to the enthalpy of melting $(\mathrm{kJ} / \mathrm{mol})$

In the UPPER scheme (Figure 1.1), the melting points are usually not known and they are calculated from the enthalpy of melting and not vice-versa. Therefore, enthalpy of melting cannot be estimated using the entropy of melting and melting points. The estimation using group contributions helps maintain the integrity of the UPPER scheme. This method is also useful in cases where melting temperature and entropy of melting data are unavailable.

\section{DATA}

For the development of a group contribution scheme, experimental melting points, and entropies of melting were collected from the literature. The majority of the experimental melting points and all of the entropy values were obtained from Chickos et al., 1999. Melting points for disubstituted benzenes were obtained from Katritzky et al., 1997. Melting points for drugs, herbicides and steroids were obtained from the electronic and print literature (Merck Index, 1996, Howard et al., 1997, Chickos et al., 2002, Chemfinder, 2004, MPBPWIN v 1.41). Compounds not included in the database are the polyalicyclic cage-like compounds such as adamantane, and compounds with reported enthalpy or entropy values that are less than $1.0 \mathrm{~kJ} / \mathrm{mol}$ or $8.0 \mathrm{~J} / \mathrm{K} . \mathrm{mol}$ respectively. 


\section{MODEL}

\section{Group Definition}

The molecular fragmentation scheme for calculating the enthalpy of melting is based on the concept of an isolating carbon. This concept was introduced by Hansch (1979) and has been successfully used in the fragmentation scheme of ClogP (Leo, 1993). An isolating carbon is a carbon that is not doubly or triply bonded to a hetero-atom. Each molecular fragment is defined as the smallest group of atoms (consisting of all carbons, hydrogen, and hetero atoms, including their non bonded electrons) that are not separated by an isolating carbon. The use of this concept allows us to identify a wide array of molecular fragments ranging from single atoms to large polyatomic groups. For example, single atoms like $\mathrm{C}, \mathrm{N}, \mathrm{O}$ and small functionalities like $-\mathrm{CH}_{3}$ and $-\mathrm{NH}_{2}$ are defined as individual groups. Fragments like $>\mathrm{SO}_{2} \mathrm{NHC}(=\mathrm{O}) \mathrm{NH}-$ of the sulfonylureas and $\mathrm{C}(=\mathrm{O}) \mathrm{NHC}(=\mathrm{O}) \mathrm{NHC}(=\mathrm{O})$ - of the barbiturates are also individual groups because there are no isolating carbons in these fragments, as seen in figures 2.1(a) and 2.1(b). Based on this definition, 128 groups are identified in this dataset of 2230 compounds. 


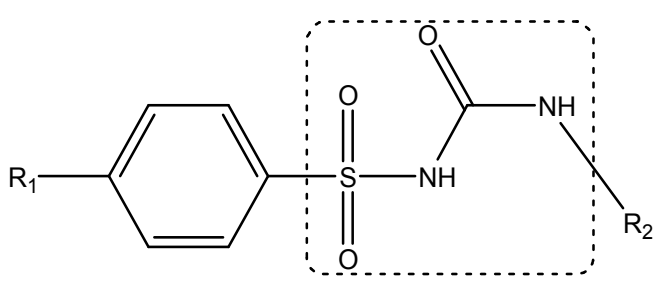

\begin{tabular}{lll} 
Sulfonylurea & $\mathrm{R}_{1}$ & $\mathrm{R}_{2}$ \\
\hline Tolazamide & $\mathrm{CH}_{3}$ & $\mathrm{C}_{6} \mathrm{H}_{12} \mathrm{~N}$ \\
Acetohexamide & $\mathrm{C}(=\mathrm{O}) \mathrm{CH}_{3}$ & $\mathrm{C}_{6} \mathrm{H}_{11}$ \\
Tolbutamide & $\mathrm{CH}_{3}$ & $\left(\mathrm{CH}_{2}\right)_{3} \mathrm{CH}_{3}$ \\
Chlorpropamide & $\mathrm{Cl}$ & $\mathrm{CH}_{2} \underline{\mathrm{CH}}_{2} \underline{\mathrm{CH}_{3}}$
\end{tabular}

Figure 2.1(a). Sulfonylureas

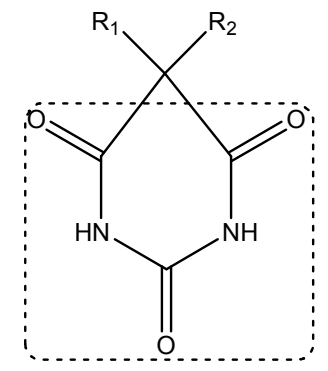

\begin{tabular}{lll} 
Barbiturate & $\mathrm{R}_{1}$ & $\mathrm{R}_{2}$ \\
\hline Amobarbital & $\mathrm{C}_{2} \mathrm{H}_{5}$ & $\left(\mathrm{CH}_{3}\right)_{2} \mathrm{CHCH}$ \\
Aprobarbital & $\mathrm{CH}_{2}=\mathrm{CHCH}_{2}$ & $\left(\mathrm{CH}_{3}\right)_{2} \mathrm{CH}$ \\
Butabarbital & $\mathrm{C}_{2} \mathrm{H}_{5}$ & $\mathrm{CH}_{3} \mathrm{CH}_{2} \mathrm{CH}\left(\mathrm{CH}_{3}\right)$ \\
Butalbital & $\mathrm{CH}_{2} \mathrm{CH}=\mathrm{CH}_{2}$ & $\mathrm{CH}_{2} \mathrm{CH}(\mathrm{CH})_{2}$ \\
Heptabarbital & $\mathrm{C}_{2} \mathrm{H}_{5}$ & $\mathrm{C}_{7} \mathrm{H}_{11}$ \\
Phenobarbital & $\mathrm{C}_{2} \mathrm{H}_{5}$ & $\mathrm{C}_{6} \mathrm{H}_{5}$ \\
Secobarbital & $\mathrm{CH}_{2} \mathrm{CH}=\mathrm{CH}_{2}$ & $\mathrm{CH}_{3}\left(\mathrm{CH}_{2}\right)_{2} \mathrm{CH}_{2}\left(\mathrm{CH}_{3}\right)$ \\
Talbutal & $\mathrm{CH}_{2} \underline{\mathrm{CH}=\mathrm{CH}_{2}}$ & $\mathrm{CH}\left(\mathrm{CH}_{3}\right) \mathrm{CH}_{2} \underline{\mathrm{CH}_{3}}$ \\
\hline
\end{tabular}

Figure 2.1(b). Barbiturates

\section{Group Environment}

The molecular environment of each constituent fragment plays an important role in determining its contribution to the total enthalpy of melting of a compound. The following eight descriptors are used to describe the different molecular environment: 
$\mathrm{X}$ - A group attached only to $\mathrm{sp}^{3}$ hybrid atoms

Y - A group attached to one, and only one, $\mathrm{sp}^{2}$ hybrid atom

YY - A group attached to two $\mathrm{sp}^{2}$ hybrid atoms

ar - atoms and groups in an aromatic ring

br -atom joining two aromatic rings

bp - biphenyl carbon

ring -atom and groups in an aliphatic ring

fus - atom joining two aliphatic rings

A pictorial representation of these molecular environments is shown in figure 2.2.

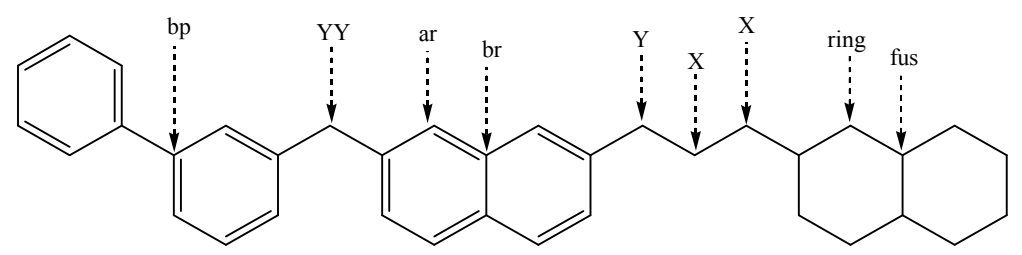

Figure2.2. Molecular environment descriptors in the fragmentation scheme

Proximity factors

In addition to the various molecular fragments, proximity factors for intramolecular hydrogen bonding, geminal halogen atoms and $-\mathrm{NH}_{2}$ and $-\mathrm{OH}$ groups on secondary and tertiary carbons are included in the enthalpy calculation.

Some ortho disubstituted aromatic compounds, as well as some disubstituted trans compounds, can form intramolecular hydrogen bonds. An intramolecular hydrogen bond would reduce the interaction of a molecule with its neighboring molecules, resulting in a 
reduced enthalpy of melting and melting point. The proximity factors IHB5, IHB6 and IHB7 are included in our model to account for five, six or seven member intramolecular hydrogen bonded ring formation as shown in figure 2.3. Also shown in the figure is the possibility of a four member intramolecular hydrogen bonded ring. But such ring formation is highly unlikely and there are greater chances of reinforced intermolecular hydrogen bonding resulting in dimerization (Simmamora et al., 1994). Therefore, IHB4 was not considered in our study. In fact, the reinforced hydrogen bonding effect is incorporated in the group contribution values of $-\mathrm{COOH}$ and $-\mathrm{CONH}$ groups. While assigning the number of possible IHB rings, compounds with two hydrogen donors are given a value of 2 as compared to a count of 1 for compounds with one hydrogen donor. For example, in o-dihydroxybenzene there is a probability for formation of 2 IHB5 rings as compared to a single IHB5 ring in o-chlorophenol.

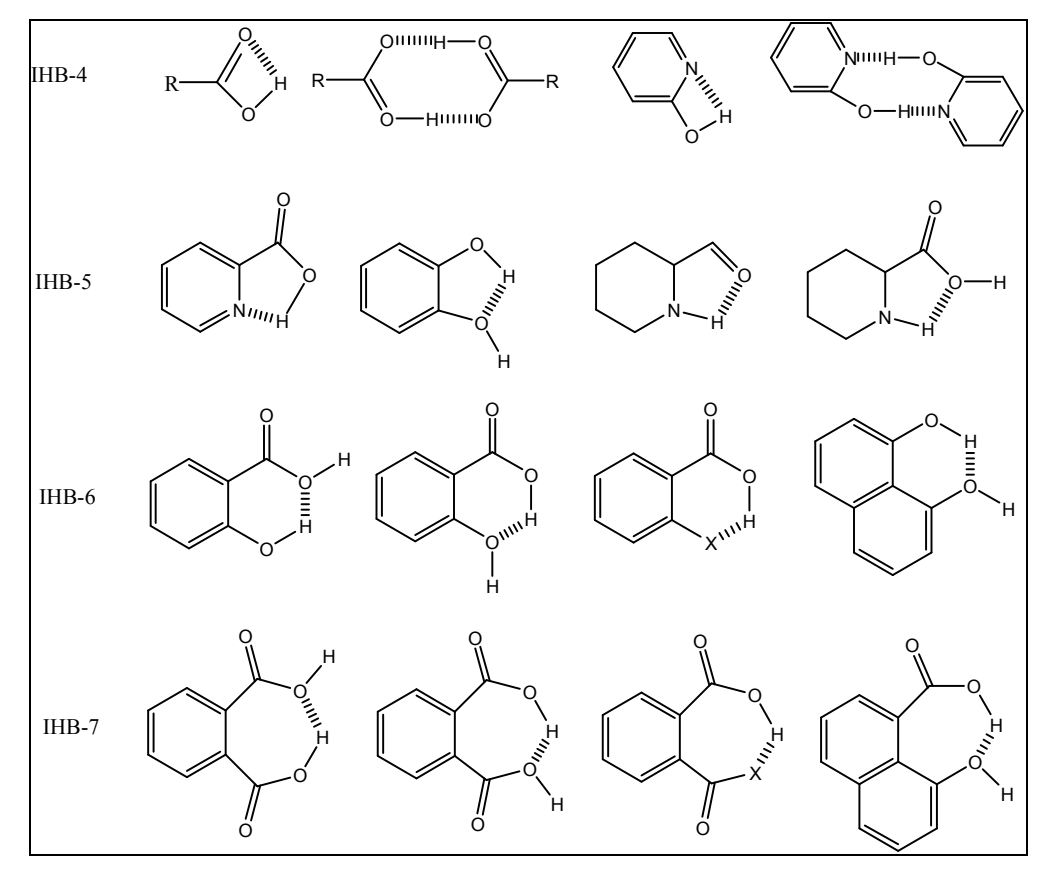

Figure2.3. Intramolecular hydrogen bonding patterns 
Three proximity factors are included in the model to account for geminal halogen atoms. A single $\mathrm{sp}^{3}$ carbon atom containing two, three or four halogen atoms tends to have a reduced enthalpy contribution. This is probably due to the repulsion between the halogen atoms. G2, G3 and G4 are the proximity factors representing a $\mathrm{sp}^{3}$ carbon containing two, three or four halogen atoms, respectively. Similar proximity factors are included for the steric effects that are associated with $-\mathrm{OH}$ and $-\mathrm{NH}_{2}$ groups on secondary and tertiary carbons.

\section{METHODS}

Group contribution values were generated using the enthalpy values obtained from the product of experimental melting points and entropies of melting. Contribution values for both the molecular fragments and proximity factors $\left(\mathrm{m}_{\mathrm{i}}\right.$ and $\left.\mathrm{m}_{\mathrm{j}}\right)$ were obtained by multiple linear regressions using SPSS Version 12.0 (SPSS Inc., Chicago, IL).

\section{RESULTS AND DISCUSSION}

Multiple linear regression for the enthalpic contribution values of 2230 compounds gives an R-squared value of 0.993 and a standard error of $2.91 \mathrm{~kJ} / \mathrm{mol} .209$ enthalpic contribution values are obtained for the groups, their molecular environment and the proximity factors. Table 2.1 gives a list of all the molecular fragments and proximity factors along with their group contribution values. Each row represents a group and each column represents the group environment. 
Table 2.1. A list of molecular fragments and proximity factors along with their enthalpy contribution values

\begin{tabular}{|c|c|c|c|c|c|c|c|c|c|}
\hline Groups & All & YY & $\mathbf{Y}$ & $\mathbf{X}$ & ar & br & bp & ring & fus \\
\hline$-\mathrm{CH}_{3}$ & ----- & ----- & 3.24 & 1.76 & ----- & ----- & ----- & ----- & ---- \\
\hline$-\mathrm{CH}_{2}$ & ----- & 2.09 & 2.10 & 2.95 & ----- & ----- & ----- & 2.33 & ----- \\
\hline$=\mathrm{CH}_{2}$ & ----- & ----- & 1.30 & ----- & ----- & ----- & ----- & ----- & ----- \\
\hline$>\mathrm{CH}-$ & ----- & -0.30 & 0.67 & -0.47 & 1.59 & ----- & ----- & -0.08 & -1.80 \\
\hline$=\mathrm{CH}-$ & ----- & ----- & 2.67 & -0.67 & ----- & ----- & ----- & 1.54 & ----- \\
\hline$\equiv \mathrm{CH}$ & ----- & ----- & 4.44 & ----- & ----- & ----- & ----- & ----- & ----- \\
\hline$>\mathrm{C}<$ & ----- & -3.02 & 0.43 & 1.19 & -0.86 & 0.18 & -0.58 & -1.93 & -2.28 \\
\hline$=\mathrm{C}<$ & ----- & -3.68 & ----- & -1.60 & ----- & ----- & ----- & -0.21 & -2.28 \\
\hline$=\mathrm{C}=$ & ----- & 0.46 & ----- & ----- & ----- & ----- & ----- & ----- & ----- \\
\hline$-C \equiv$ & ----- & ----- & 0.65 & -1.59 & ----- & ----- & ----- & ----- & ----- \\
\hline$-F$ & ----- & ----- & 2.47 & 2.03 & ----- & ----- & ----- & ----- & ----- \\
\hline$-\mathrm{Cl}$ & ----- & ----- & 3.48 & 3.85 & ----- & ----- & ----- & ----- & ----- \\
\hline$-B r$ & ----- & ----- & 4.47 & 4.67 & ----- & ----- & ----- & ----- & ----- \\
\hline -I & ----- & ----- & 5.43 & 6.58 & ----- & ----- & ----- & ----- & ----- \\
\hline$>\mathrm{N}-$ & ----- & -8.66 & 1.67 & 1.25 & 2.39 & 1.90 & ----- & 0.34 & ----- \\
\hline -O- & ----- & 1.67 & 4.55 & 1.57 & 1.68 & ----- & ----- & 4.32 & ----- \\
\hline -S- & ----- & 1.08 & 4.09 & 2.81 & 3.19 & ----- & ----- & 6.79 & ----- \\
\hline -CN & ----- & ----- & 6.58 & 6.09 & ----- & ----- & ----- & ----- & ----- \\
\hline$>\mathrm{C}(=\mathrm{O})$ & ----- & 0.38 & 3.38 & 2.35 & 4.93 & ----- & ----- & 4.28 & ----- \\
\hline$>\mathrm{NH}$ & ----- & 6.90 & 6.14 & 4.71 & 8.28 & ----- & ----- & 3.38 & ----- \\
\hline$-\mathrm{N}=\mathrm{N}-$ & ----- & 1.63 & ----- & ----- & 2.82 & ----- & ----- & ----- & ----- \\
\hline -NO- & ----- & ----- & ----- & ----- & 3.29 & ----- & ----- & ----- & ----- \\
\hline -NS- & ----- & ----- & ----- & ----- & 5.64 & ----- & ----- & ----- & ----- \\
\hline$-\mathrm{OH}$ & ----- & ----- & 6.16 & 5.21 & ----- & ----- & ----- & ----- & ----- \\
\hline -SH & ----- & ----- & 4.34 & 1.85 & ----- & ----- & ----- & ----- & ----- \\
\hline$>\mathrm{S}(=\mathrm{O})$ & 6.42 & ----- & ----- & ----- & ----- & ----- & ----- & ----- & ----- \\
\hline$-\mathrm{P}(=\mathrm{O})<$ & -3.59 & ----- & ----- & ----- & ----- & ----- & ----- & ----- & ----- \\
\hline -SS- & 4.80 & ----- & ----- & ----- & ----- & ----- & ----- & ----- & ----- \\
\hline$-\mathrm{N}_{2} \mathrm{~S}-$ & ----- & ----- & ----- & ----- & 8.04 & ----- & ----- & ----- & ----- \\
\hline$-\mathrm{NH}_{2}$ & ----- & ----- & 5.96 & 4.14 & ----- & ----- & ----- & ----- & ----- \\
\hline$-\mathrm{NO}_{2}$ & ----- & ----- & 5.89 & 4.86 & ----- & ----- & ----- & 9.34 & ----- \\
\hline$-\mathrm{SO}_{2^{-}}$ & ----- & ----- & 4.21 & 8.06 & ----- & ----- & ----- & -2.84 & ----- \\
\hline$-\mathrm{SO}_{3}^{-}$ & 4.15 & ----- & ----- & ----- & ----- & ----- & ----- & ----- & ----- \\
\hline$-\mathrm{C}(=\mathrm{O}) \mathrm{O}-$ & ----- & ---- & 6.05 & 4.04 & 7.95 & ---- & ---- & 7.15 & ---- \\
\hline
\end{tabular}


Table 2.1. Cont..

\begin{tabular}{|c|c|c|c|c|c|c|c|c|c|}
\hline Groups & All & YY & $\mathbf{Y}$ & $\mathbf{X}$ & ar & br & bp & ring & fus \\
\hline$-\mathrm{CH}=\mathrm{N}$ & -2.13 & ----- & ----- & ----- & ----- & ----- & ---- & ----- & ---- \\
\hline$-\mathrm{CH}=\mathrm{O}$ & ----- & ----- & 5.88 & 5.54 & ----- & ----- & ----- & ----- & ----- \\
\hline$-\mathrm{C}(=\mathrm{O}) \mathrm{N}<$ & ----- & ----- & 0.19 & 3.51 & ----- & ----- & ----- & 8.80 & ----- \\
\hline$-\mathrm{SS}(=\mathrm{O})-$ & 12.90 & ----- & ----- & ----- & ----- & ----- & ----- & ----- & ----- \\
\hline$-\mathrm{N}(=\mathrm{O})=\mathrm{N}-$ & 4.72 & ----- & ----- & ----- & ----- & ----- & ----- & ----- & ----- \\
\hline$>\mathrm{NNH}-$ & 7.33 & ----- & ----- & ----- & 11.95 & ----- & ----- & ----- & ---- \\
\hline$>\mathrm{NNH}_{2}$ & 7.28 & ----- & ----- & ----- & ----- & ----- & ----- & ----- & ----- \\
\hline$>\mathrm{NN}=\mathrm{O}$ & 7.17 & ---- & ---- & ---- & ---- & ----- & ----- & ----- & ----- \\
\hline$>\mathrm{NNO}_{2}$ & 8.11 & ----- & ----- & ----- & ----- & ----- & ----- & ----- & ----- \\
\hline$-\mathrm{OC} \equiv \mathrm{N}-$ & 7.95 & ----- & ----- & ----- & ----- & ----- & ---- & ----- & ---- \\
\hline$-\mathrm{ONO}_{2}$ & 7.44 & ----- & ----- & ----- & ----- & ----- & ---- & ----- & ----- \\
\hline $\mathrm{N} \equiv \mathrm{CC} \equiv \mathrm{N}$ & 10.86 & ----- & ----- & ----- & ----- & ----- & ----- & ---- & ----- \\
\hline$>\mathrm{NCH}=\mathrm{O}$ & 7.91 & ----- & ----- & ----- & ----- & ----- & ---- & ----- & ----- \\
\hline$-\mathrm{OCH}=\mathrm{O}$ & 4.21 & ----- & ----- & ----- & ----- & ----- & ----- & ----- & ----- \\
\hline$-\mathrm{NH} 2 \mathrm{C} \equiv \mathrm{N}$ & 9.80 & ----- & ----- & ----- & ----- & ----- & ---- & ----- & ----- \\
\hline -NHNH- & 11.93 & ----- & ----- & ----- & ----- & ----- & ----- & ----- & ----- \\
\hline$-\mathrm{NHNH}_{2}$ & 8.96 & ----- & ----- & ----- & ----- & ----- & ----- & ----- & ----- \\
\hline$-\mathrm{NHNO}_{2}$ & 11.91 & ----- & ----- & ----- & ----- & ----- & ---- & ----- & ---- \\
\hline$-\mathrm{NN}(\mathrm{R}) \mathrm{C}(=\mathrm{O})-$ & ----- & ----- & ----- & ----- & 13.06 & ----- & ----- & ----- & ----- \\
\hline$-\mathrm{NNN}(\mathrm{R}) \mathrm{C}(=\mathrm{O})-$ & ---- & ----- & ---- & ---- & 4.61 & ----- & ----- & ----- & ----- \\
\hline -NN(R)NN- & ----- & ----- & ----- & ----- & 8.03 & ----- & ----- & ----- & ----- \\
\hline -NNNNH- & ----- & ----- & ---- & ---- & 16.82 & ----- & ---- & ---- & ---- \\
\hline$>\mathrm{C}=\mathrm{NOH}$ & 6.06 & ----- & ----- & ----- & ----- & ----- & ---- & ----- & ---- \\
\hline$-\mathrm{CH}=\mathrm{NO}-$ & ----- & ----- & ----- & ----- & ---- & ----- & ----- & 6.78 & ----- \\
\hline$-\mathrm{CH}=\mathrm{NOH}$ & 9.65 & ----- & ---- & ----- & ----- & ----- & ---- & ----- & ---- \\
\hline$-\mathrm{C}(=\mathrm{O}) \mathrm{OH}$ & ----- & ----- & 12.15 & 9.91 & ----- & ----- & ----- & ----- & ---- \\
\hline$-\mathrm{C}(=\mathrm{O}) \mathrm{Cl}$ & 5.45 & ----- & ----- & ----- & ----- & ----- & ---- & ----- & ---- \\
\hline$-\mathrm{C}(=\mathrm{O}) \mathrm{C}(=\mathrm{O})-$ & 6.85 & ----- & ----- & ----- & ---- & ----- & ----- & ---- & ----- \\
\hline$-\mathrm{CH}=\mathrm{N}-\mathrm{N}=\mathrm{CH}-$ & 6.75 & ----- & ----- & ---- & ----- & ----- & ----- & ---- & ----- \\
\hline$-\mathrm{C}(=\mathrm{O}) \mathrm{NH}-$ & ----- & ----- & 10.59 & 11.14 & 9.63 & ---- & ----- & 14.05 & ---- \\
\hline$-\mathrm{C}(=\mathrm{O}) \mathrm{NH}_{2}$ & ---- & ---- & 12.90 & 12.92 & ----- & ----- & ---- & ---- & ---- \\
\hline$-\mathrm{C}(=\mathrm{S}) \mathrm{NH}_{2}$ & 16.04 & ----- & ---- & ---- & ---- & ----- & ---- & ---- & ---- \\
\hline -NHC $(=\mathrm{O}) \mathrm{NH}-$ & 17.57 & ----- & ----- & ----- & ----- & ----- & ----- & ----- & ---- \\
\hline -NHC $(=\mathrm{S}) \mathrm{NH}-$ & 20.23 & ---- & ---- & ---- & ---- & ---- & --- & ---- & $-\cdots$ \\
\hline
\end{tabular}


Table 2.1. Cont..

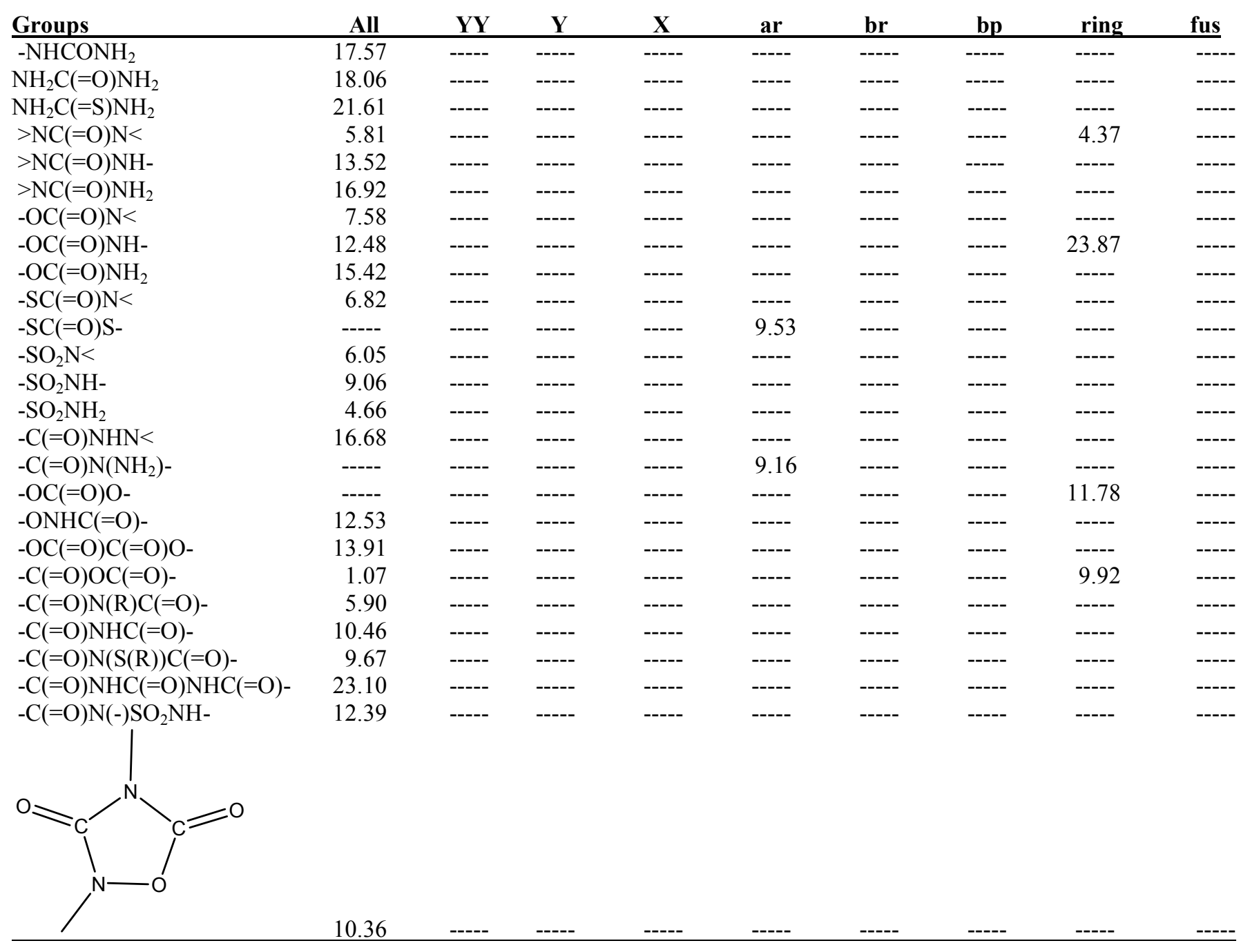


Table 2.1. Cont..

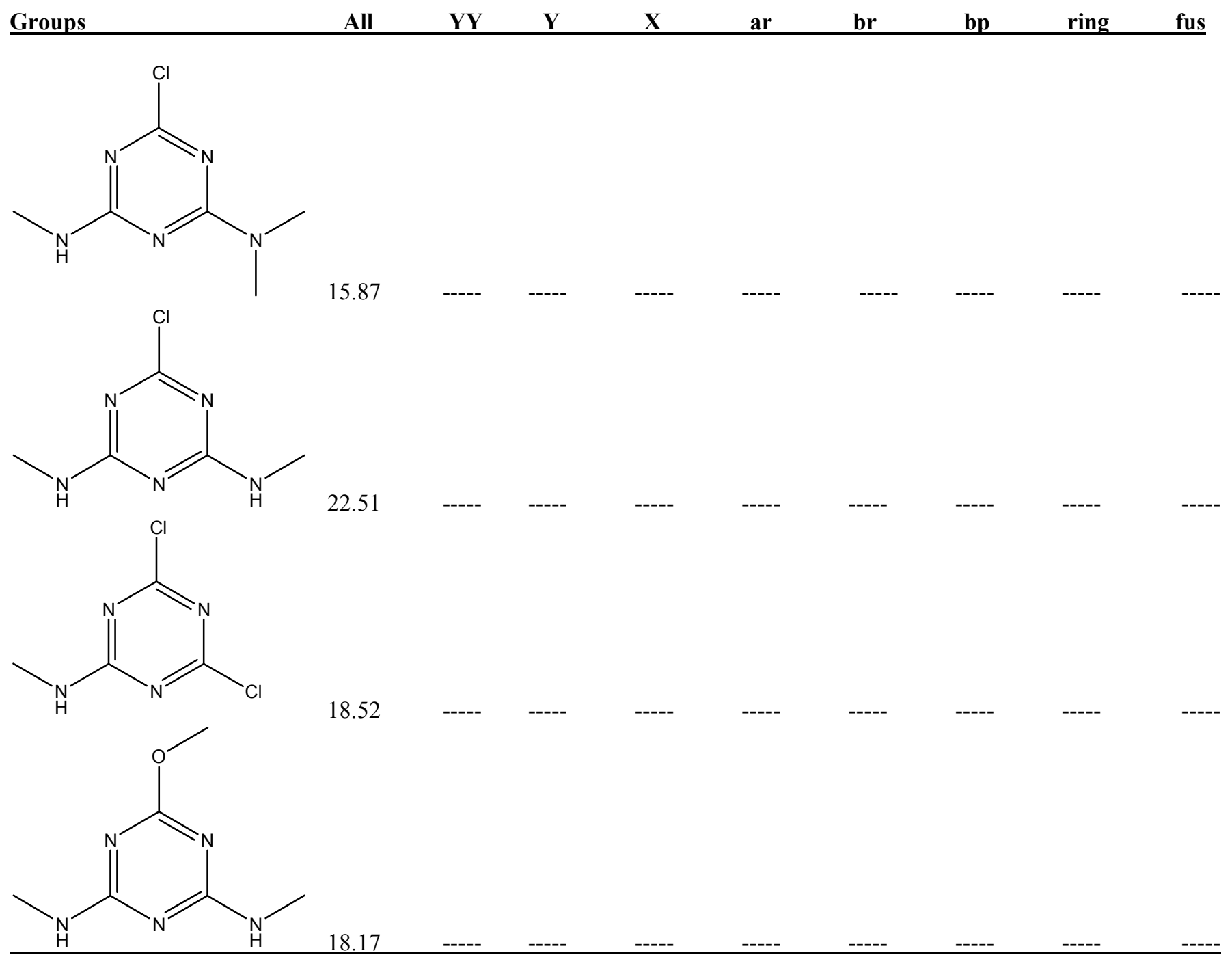


Table 2.1. Cont..

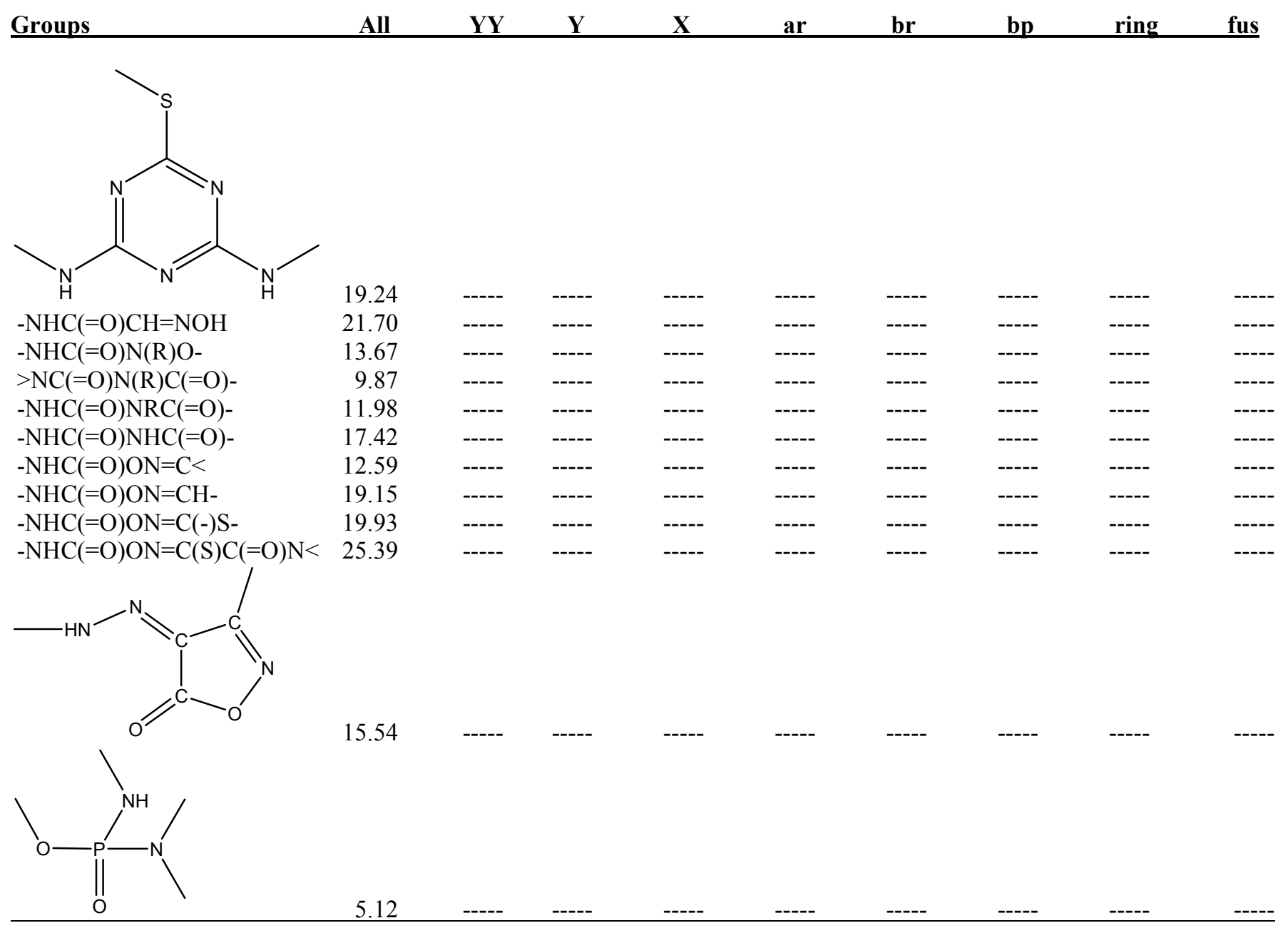


Table 2.1. Cont..

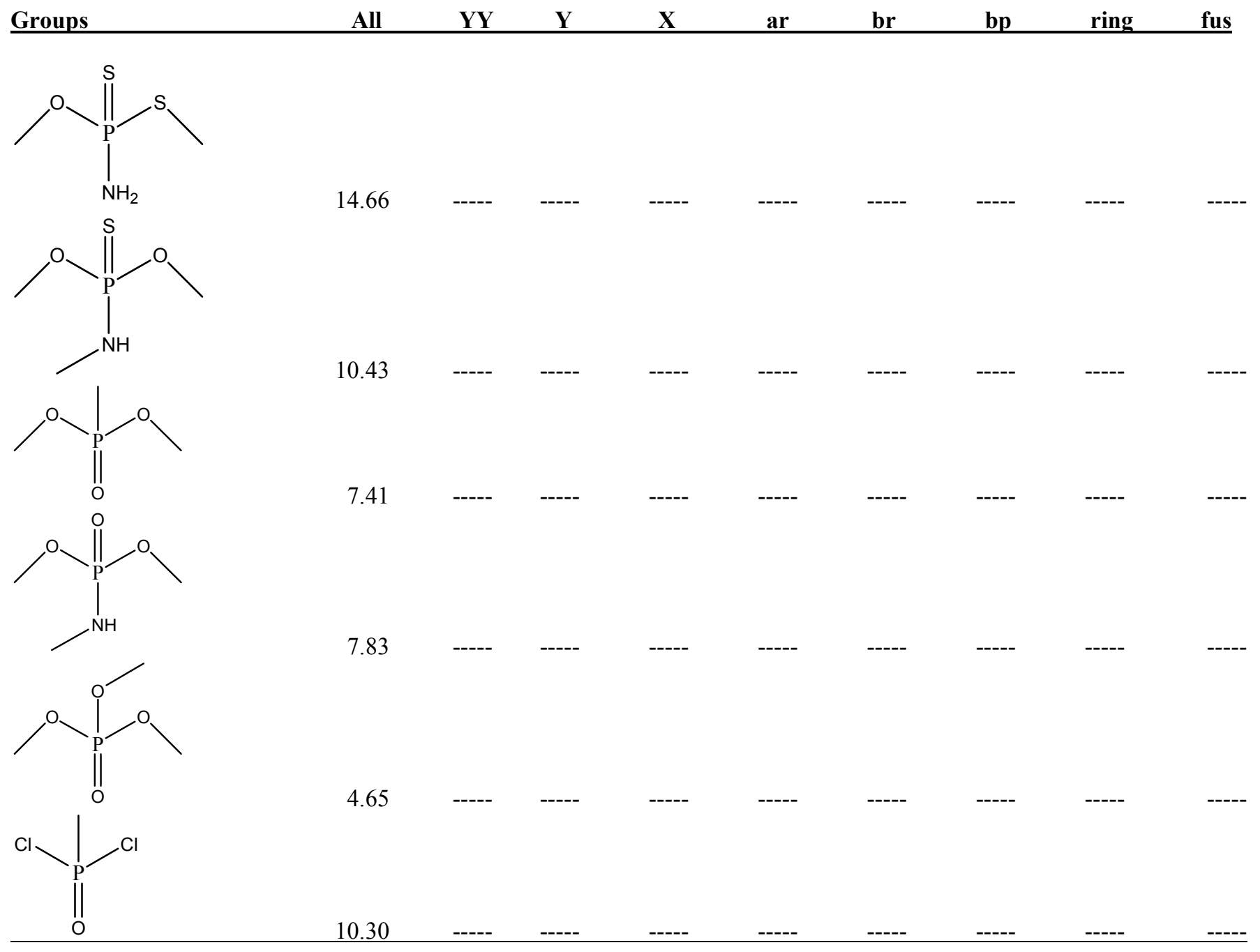


Table 2.1. Cont..

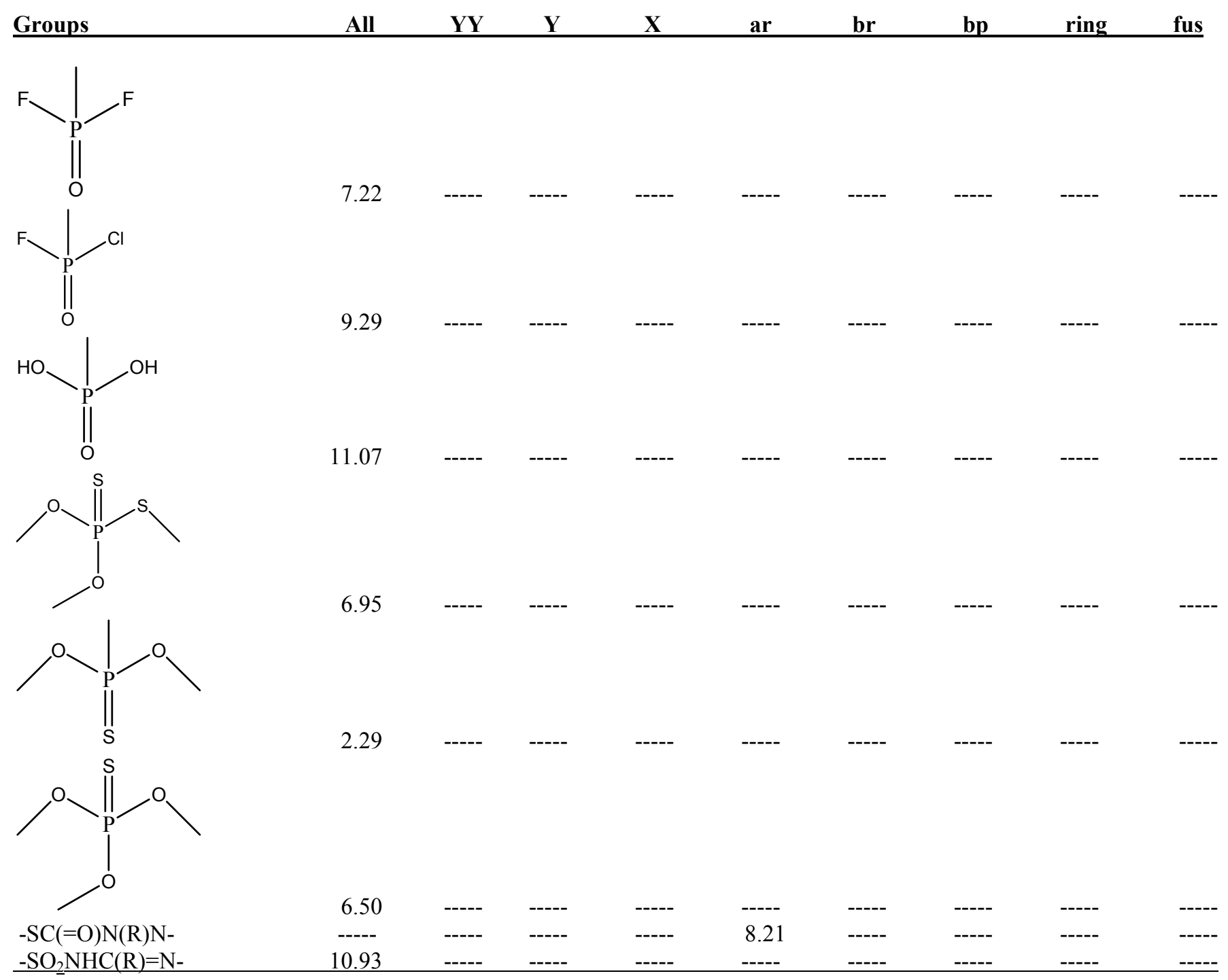


Table 2.1. Cont..

\begin{tabular}{|c|c|c|c|c|c|c|c|c|c|}
\hline Groups & All & YY & $\mathbf{Y}$ & $\mathbf{X}$ & ar & br & bp & ring & fus \\
\hline$-\mathrm{SO}_{2} \mathrm{NHCH}=\mathrm{N}-$ & 20.58 & $\begin{array}{ll}---- \\
\end{array}$ & $\begin{array}{ll}---- \\
\end{array}$ & $\begin{array}{ll}---- \\
\end{array}$ & $\begin{array}{ll}---- \\
-1\end{array}$ & ----- & $\begin{array}{ll}---- \\
--\end{array}$ & $\overrightarrow{-----}$ & $\overline{---}$ \\
\hline$-\mathrm{SO}_{2} \mathrm{NHC}(=\mathrm{O})-$ & 12.97 & ----- & ----- & ----- & ----- & ----- & ----- & ----- & ---- \\
\hline$-\mathrm{SO}_{2} \mathrm{NHC}(=\mathrm{O}) \mathrm{NH}-$ & 14.71 & ----- & ----- & ----- & ----- & ----- & ----- & ----- & ---- \\
\hline$-\mathrm{SO}_{2}{ }_{2} \mathrm{NHC}(=\mathrm{O}) \mathrm{NHN}<$ & 21.56 & ----- & ----- & ----- & ----- & ----- & ----- & ----- & $---\cdot$ \\
\hline \multicolumn{10}{|l|}{ Proximity factors } \\
\hline$\overline{\mathrm{IHB} 5}$ & -0.19 & ----- & ----- & ----- & ----- & ----- & ----- & ----- & --- \\
\hline IHB6 & -1.30 & ----- & ----- & ----- & ----- & ----- & ----- & ----- & ---- \\
\hline IHB7 & -0.64 & ----- & ----- & ----- & ----- & ----- & ----- & ----- & --- \\
\hline G2 & -1.02 & ----- & ----- & ----- & ----- & ----- & ----- & ----- & --- \\
\hline G3 & -4.64 & ----- & ----- & ----- & ----- & ----- & ----- & ----- & --- \\
\hline G4 & -7.50 & ----- & ----- & ----- & ----- & ----- & ----- & ----- & --- \\
\hline $2^{\circ} \mathrm{OH}$ & 5.16 & ----- & ----- & ----- & ----- & ----- & ----- & ----- & ---- \\
\hline $3^{\circ} \mathrm{OH}$ & 4.25 & ----- & ----- & ----- & ----- & ----- & ----- & ----- & --- \\
\hline $2^{\circ} \mathrm{NH}_{2}$ & 7.16 & ----- & ----- & ----- & ----- & ----- & ----- & ----- & --- \\
\hline $3^{\circ} \mathrm{NH}_{2}$ & 3.54 & ----- & ----- & ----- & ----- & ----- & ----- & ----- & --- \\
\hline
\end{tabular}

Note: In the table, $R$ represents an alkyl linkage 
The average absolute error in predicting the enthalpy of melting for 1663 compounds using equation (2.1) is $5.5 \mathrm{~kJ} / \mathrm{mol}$. It is to be noted that the group contribution values were generated from the entire database of 2230 compounds. However, the errors are calculated for the 1663 compounds with reported enthalpy values. For the remaining compounds with no experimental enthalpy and entropy data, the enthalpy values were generated from the product of experimental melting points and our calculated entropy values. These values were used solely for generating group contributions by multiple regression.

The error distribution obtained while correlating the experimental and predicted enthalpy values which range from $2.3 \mathrm{~kJ} / \mathrm{mol}$ to $698.9 \mathrm{~kJ} / \mathrm{mol}$ is shown in figure 2.4 .

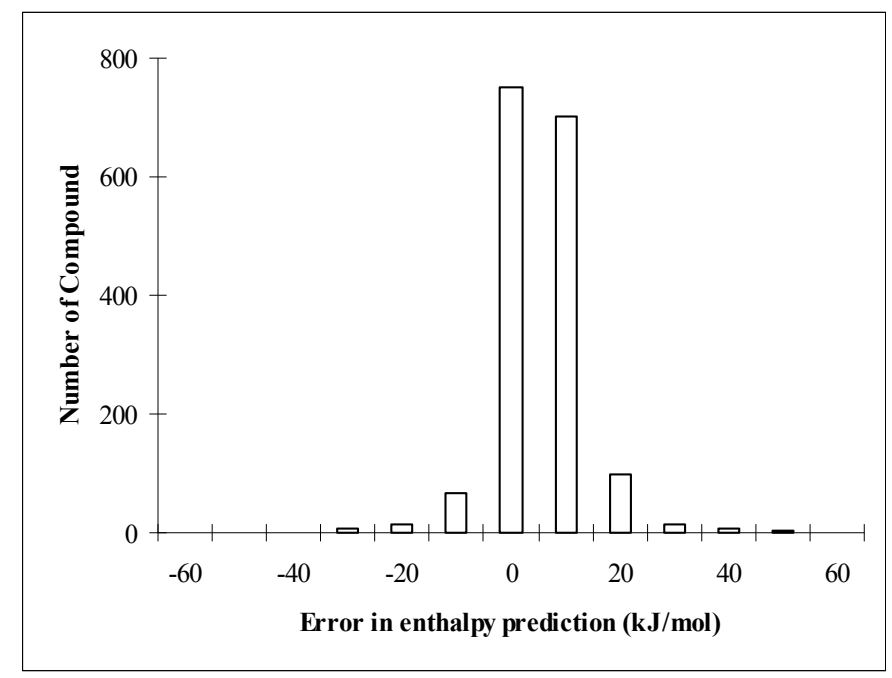

Figure2.4. The distribution of errors in predicting enthalpy of melting 
The error distribution in figure 2.4 is fairly symmetrical with a very few outliers. A frequency distribution of the average absolute errors in the prediction of enthalpy of melting is shown in Table 2.2. More than $87 \%$ of the compounds have average absolute errors less than $10.0 \mathrm{~kJ} / \mathrm{mol}$.

Table 2.2. Frequency distribution of the average absolute errors in enthalpy of melting prediction

\begin{tabular}{lcc} 
Average absolute & Number of & Cumulative \\
\hline Error (kJ/mol) & compounds & \% total \\
\hline Below 10 & 1449 & 87 \\
$10-20$ & 168 & 97 \\
Above 20 & 46 & 100 \\
\hline
\end{tabular}

Two examples for predicting enthalpies of melting using the proposed model are shown in Appendix A. A complete list of experimental and calculated enthalpies of melting for the 1663 compounds is given in Appendix B. All compounds are listed in alphanumeric order.

\section{SUMMARY}

A group contribution model for calculating enthalpy of melting is developed. The group contributions are generated from a large, complex dataset of over twenty-two hundred organic compounds. The model predicts the enthalpy of melting for 1663 organic compounds which range from $2.3 \mathrm{~kJ} / \mathrm{mol}$ to $698.9 \mathrm{~kJ} / \mathrm{mol}$ with an average absolute error of $5.5 \mathrm{~kJ} / \mathrm{mol}$. The calculation is simple as it requires only the SMILES notation of a compound as the input parameter. The inclusion of different molecular environment and proximity factors contribute to the accuracy of this model. This model is based on the 
isolating carbon rule that distinguishes the large complex molecular fragments that are usually present in drugs and environmental chemicals. 


\section{CHAPTER 3: ENTROPY OF MELTING}

\section{INTRODUCTION}

Total entropy of melting $\left(\Delta \mathrm{S}_{\mathrm{m}}^{\mathrm{tot}}\right)$ includes contributions from all solid-solid transitions and the solid-liquid transition. Unlike the enthalpy of melting, it is better understood in geometric terms. By definition, the entropy change associated with melting is related to the relative probabilities of existence of a group of molecules conforming to the geometric restrictions of the liquid and the solid state. Applying the Boltzman relationship to melting, it can be defined as

$$
\Delta \mathrm{S}_{\mathrm{m}}^{\mathrm{tot}}=-\mathrm{R} \ln \left(\Omega^{\mathrm{S}} / \Omega^{\mathrm{L}}\right)
$$

Where $\Omega^{\mathrm{S}}$ is the number of ways that 1 mole of a substance can be arranged within the geometric requirements of a crystal and $\Omega^{\mathrm{L}}$ is the same for a liquid. According to Bondi (1968), a crystal lattice can place translational, rotational and conformational constraints on its molecules. All of these factors should be included in the estimation of the relative probability of melting. Therefore, the total entropy of melting of a compound is given by:

$$
\Delta \mathrm{S}_{\mathrm{m}}^{\mathrm{tot}}=\Delta \mathrm{S}_{\mathrm{m}}^{\mathrm{pos}}+\Delta \mathrm{S}_{\mathrm{m}}^{\mathrm{rot}}+\Delta \mathrm{S}_{\mathrm{m}}^{\mathrm{conf}}
$$


The total entropy of melting mainly consists of the rotational and conformational components; the positional entropy change being relatively small and constant at 15.0 $\mathrm{J} / \mathrm{K}$.mol because of the consistent $10-15 \%$ increase in volume with melting for organic compounds.

A number of approaches have been used in the past for estimating the total entropy of melting of organic compounds. According to Walden's rule, the entropy of melting for rigid, aromatic compounds is constant with a value of $56.5 \mathrm{~J} / \mathrm{K}$.mol. Richard's rule states that the entropy of melting is constant for small spherical compounds (e.g., methane and neon) with a value of $10.5 \mathrm{~J} / \mathrm{K} . \mathrm{mol}$. A large number of compounds do not belong to either of the above categories and hence, cannot be reasonably estimated (Jain et al. 2004). Similar to the enthalpy of melting, group contributions have been used in the past for the estimation of entropy of melting (Kirshenbaum, 1965, Chickos et al. 1989, 1999). In their work, Chickos et al. developed a group contribution method for estimating the total entropy of melting with 144 groups. They obtained an average absolute error of 9.9 $\mathrm{J} / \mathrm{K} . \mathrm{mol}$ in predicting the entropy of melting for 1858 organic compounds. While their method has wide applicability, it is cumbersome as it employs a large number of group contribution values and values may be missing for some group fragments.

Although group contributions seem to provide sufficient accuracy in estimation of entropy of melting, entropy is better understood in terms of molecular shape and geometry. In 1979, Martin et al. and Yalkowsky introduced the effects of symmetry and flexibility on the total entropy of melting, respectively. Later, Dannenfelser et al. (1996, 1999) developed a semi-empirical equation combining the effects of rotational symmetry 
and molecular flexibility on the total entropy of melting. Their equation is based on only two non-additive molecular parameters,

$$
\Delta \mathrm{S}_{\mathrm{m}}^{\mathrm{tot}}=50-19.1 \log \sigma+19.1 \log \varphi
$$

The rotational entropy is related to the rotational symmetry number $(\sigma)$; whereas, the conformational entropy is related to the molecular flexibility number $(\varphi)$ of a compound. These are properties of the whole molecule and are not equal to the sum of the properties of the molecular fragments. They account for the changes in geometrical restriction that accompany the process of melting. In this model, the above equation is used to calculate the total entropy change upon melting.

\section{DATA}

The experimental entropies of melting used in this study are the same as described in Chapter 2.

\section{METHODS}

Molecular Rotational Symmetry Number

The rotational symmetry number accounts for the likelihood of a molecule being properly oriented for incorporation into the crystal lattice. It is defined as the number of positions into which a molecule can be rotated that are identical to a reference position (Dannenfelser et al., 1996). The rotational symmetry number is an important parameter 
for predicting melting points of isomers, e.g. the more symmetric anthracene melts nearly $120^{\circ}$ higher than its less symmetrical isomer phenanthrene. By assigning different rotational symmetry numbers for isomers such as phenanthrene $(\sigma=2)$ and anthracene $(\sigma=4)$, the model accounts for this large difference in melting points.

While assigning a value to $\sigma$, groups such as methyl, hydroxyl, mercapto and primary amines are assumed to be freely rotating and are treated as being radially symmetrical. The nitro and carboxyl groups are treated as laterally symmetrical. All other groups are assumed to be asymmetrical. The rotational symmetry number for a molecule is never less than unity because every molecule has at least one identical orientation that is produced by a $360^{\circ}$ rotation about any axis.

Conical (e.g., hydrogen cyanide and chloromethane) and cylindrical (e.g., carbon dioxide and ethane) molecules are empirically assigned symmetry numbers of 10 and 20, respectively. Spherical molecules (e.g., neon and methane) are assigned a symmetry number of 100. All flexible molecules are assigned a symmetry number of unity. Examples of rotational symmetry for a number of molecules are shown in figure 3.1. 


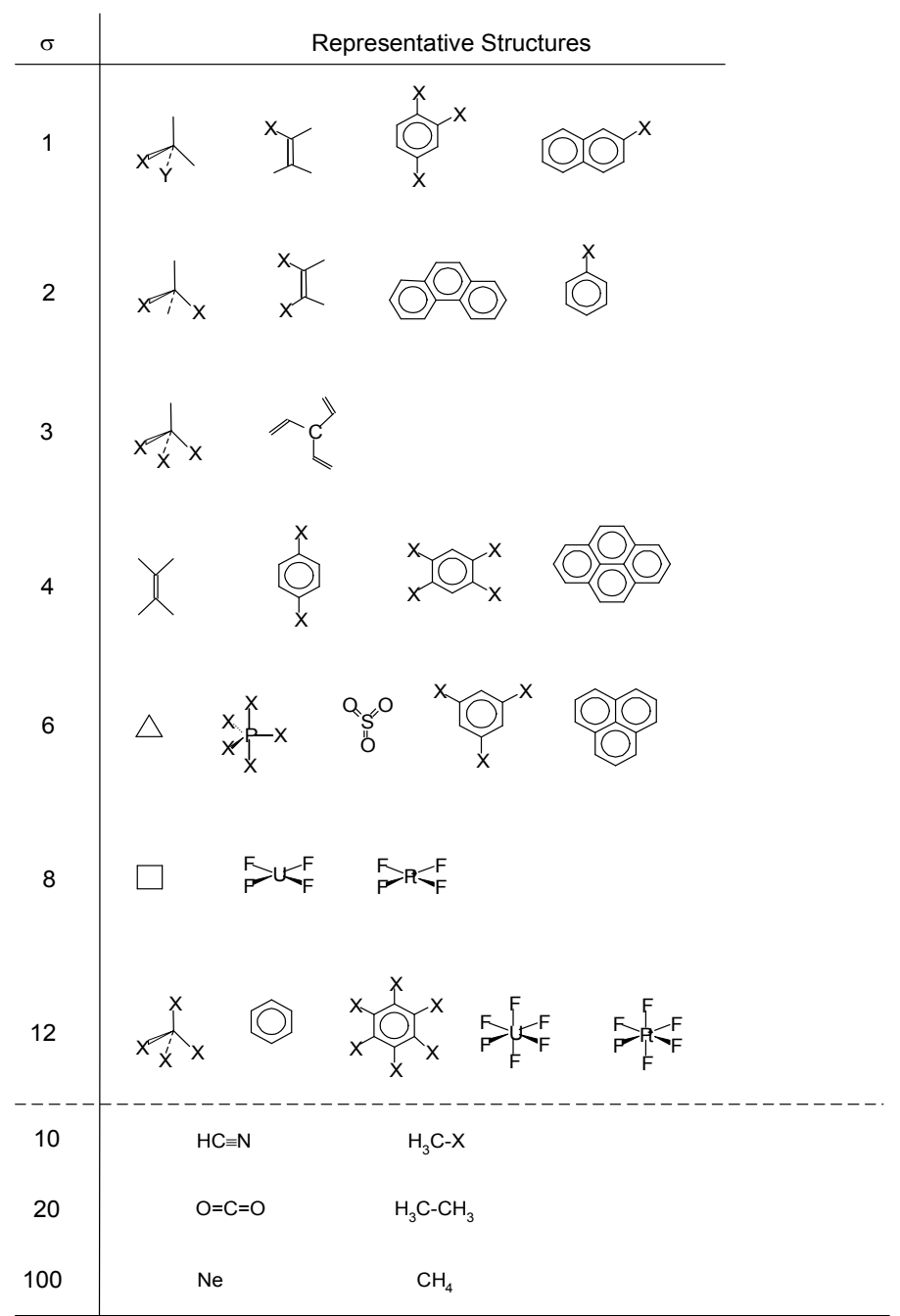

Figure 3.1. Symmetry numbers for several molecules

\section{Molecular Flexibility Number}

The molecular flexibility number $(\varphi)$ is an exponential function of chain length and is calculated by:

$$
\varphi=2.435^{\Phi}
$$


So that

$$
19.1 \log \varphi=7.4 \Phi
$$

$\Phi$ denotes the number of torsional angles or the flexibility count of a molecule. The flexibility count for any compound can be calculated using the following semi-empirical equation of Dannenfelser et al. (1999):

$$
\Phi=\mathrm{SP} 3+0.5 \mathrm{SP} 2+0.5 \mathrm{RING}-1
$$

Where $\mathrm{SP} 3=\Sigma$ Nonring $\left(\mathrm{CH}_{2}, \mathrm{CH}, \mathrm{C}, \mathrm{NH}, \mathrm{N}, \mathrm{O}, \mathrm{S}\right)$

$\mathrm{SP} 2=\Sigma$ Nonring $(=\mathrm{CH},=\mathrm{C},=\mathrm{N}, \mathrm{C}=\mathrm{O})$

Ring $=\Sigma$ Independent single, fused, or conjugated ring systems

The value 2.435 in equation 3.4 is based upon theoretical calculations by Tonelli (1970), Kirshenbaum (1965) and Starkweather et al. (1960) which state that the conformational entropy of melting for an isolated polyethylene chain is $7.4 \pm 0.2 \mathrm{~J} / \mathrm{K}$.mol per four-atom torsional unit. These calculations are based on the assumptions that the trans conformation is more stable than the gauche conformations and the gauche $(+)$ - gauche (-) sequences are hindered or forbidden.

In the flexibility count, terminal groups such as $\mathrm{CH}_{3}, \mathrm{NH}_{2}, \mathrm{OH}, \mathrm{CN}, \mathrm{F}, \mathrm{Cl}, \mathrm{Br}, \mathrm{I},=\mathrm{O}$, $=\mathrm{CH}_{2}$, and $\equiv \mathrm{N}$ as well as non-terminal sp hybrid carbons are not included. Also not included are carbon atoms with three identical groups, for example, trihalomethyl or tert- 
butyl groups. Compounds with a negative value for $\Phi$ are assigned a value of 0 . For compounds containing aliphatic cyclic rings such as cyclohexane, a value of -2 per ring is added (Jain et al., 2004). A few examples of molecular flexibility numbers are shown in Table 3.1.

Table 3.1. Examples of some molecular flexibility numbers

\begin{tabular}{llllllr} 
Name & $\boldsymbol{\sigma}$ & SP3 & SP2 & RING & $\Phi$ & \multicolumn{1}{c}{$\boldsymbol{\Phi}$} \\
\hline n-pentane & 1 & 3 & 0 & 0 & 2 & 5.93 \\
2-methylbutane & 1 & 2 & 0 & 0 & 1 & 2.44 \\
neopentane & 1 & 1 & 0 & 0 & 0 & 1.00 \\
1-pentanol & 1 & 4 & 0 & 0 & 3 & 14.44 \\
3-pentanone & 1 & 2 & 1 & 0 & 1.5 & 3.80 \\
cyclopentane & 0 & 5 & 0 & 0 & 2 & 5.93 \\
toluene & 2 & 0 & 0 & 1 & 0 & 1.00 \\
tert-butylbenzene & 2 & 0 & 0 & 1 & 0 & 1.00 \\
1,2,4 trimethylbenzene & 1 & 0 & 0 & 1 & 0 & 1.00 \\
1,3,5-trimethylbenzene & 6 & 0 & 0 & 1 & 0 & 1.00 \\
4-chlorophenol & 2 & 0 & 0 & 1 & 0 & 1.00 \\
benzoic acid & 2 & 0 & 0 & 1 & 0 & 1.00 \\
2,4,6-trinitrotoluene & 2 & 0 & 0 & 1 & 0 & 1.00 \\
n-butylbenzene & 1 & 3 & 0 & 1 & 2.5 & 9.25 \\
hexamethylbenzene & 12 & 0 & 0 & 1 & 0 & 1.00 \\
\hline
\end{tabular}

\section{RESULTS AND DISCUSSION}

The average absolute error in predicting the total entropy of melting for 1663 compounds using equation (3.3) is $15.0 \mathrm{~J} / \mathrm{K} . \mathrm{mol}$. The error distribution obtained while correlating the experimental and predicted entropy values which range from $8.5 \mathrm{~J} / \mathrm{K}$.mol to 1751.2 $\mathrm{J} / \mathrm{K} . \mathrm{mol}$ is shown in figure 3.2 . 


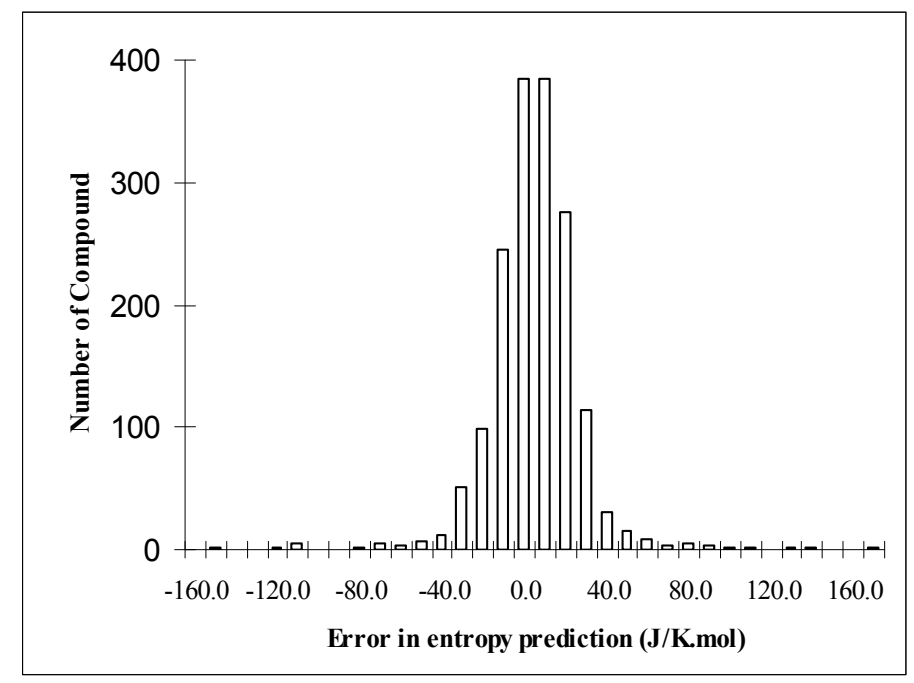

Figure3. 2. The distribution of errors in predicting entropy of melting

As seen in Figure 3.2, the error distribution is fairly symmetrical with a very few outliers. A majority of the outliers are the very long chain flexible molecules similar to those seen in enthalpy calculations. Figures 3.3 and 3.4 show the distribution of errors as a function of the logarithm of the rotational symmetry number $(\sigma)$ and molecular flexibility count (Ф). In Figure 3.3, an almost symmetric distribution of errors is observed at each rotational symmetry number. On the other hand, as seen in Figure 3.4, at low flexibility counts the errors are distributed symmetrically but as the flexibility count increases, the distribution becomes more random with a large proportion of outliers. Such long chain, flexible molecules are very likely to form liquid crystals and exhibit mesophasic behavior and multiple transitions before the actual melting to the isotropic liquid. Sometimes the entropies associated with all these transitions are not accounted for in the reported 
experimental entropy values. This leads to an increase in the average error associated with their entropy predictions.

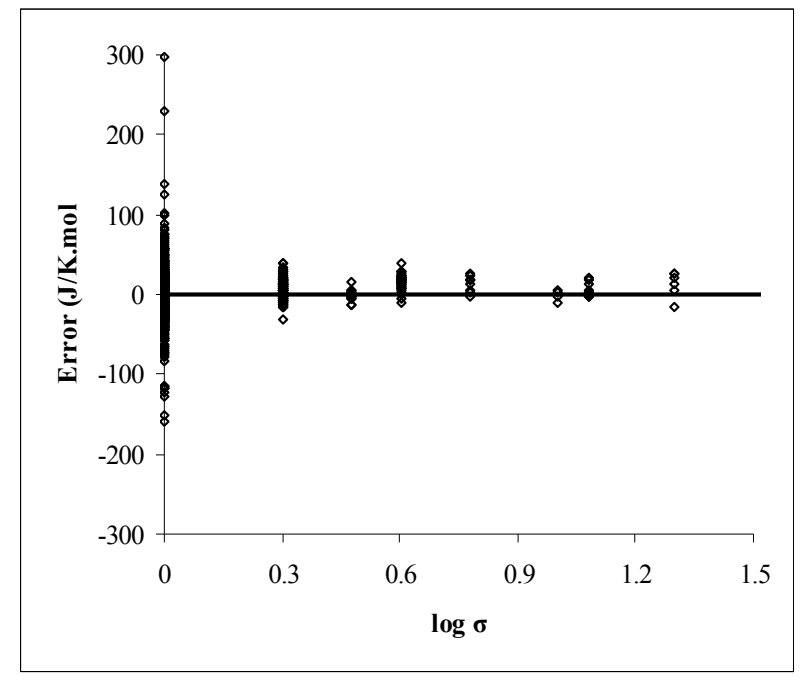

Figure 3.3. Error distribution as a function of the logarithm of the rotational symmetry number $(\sigma)$

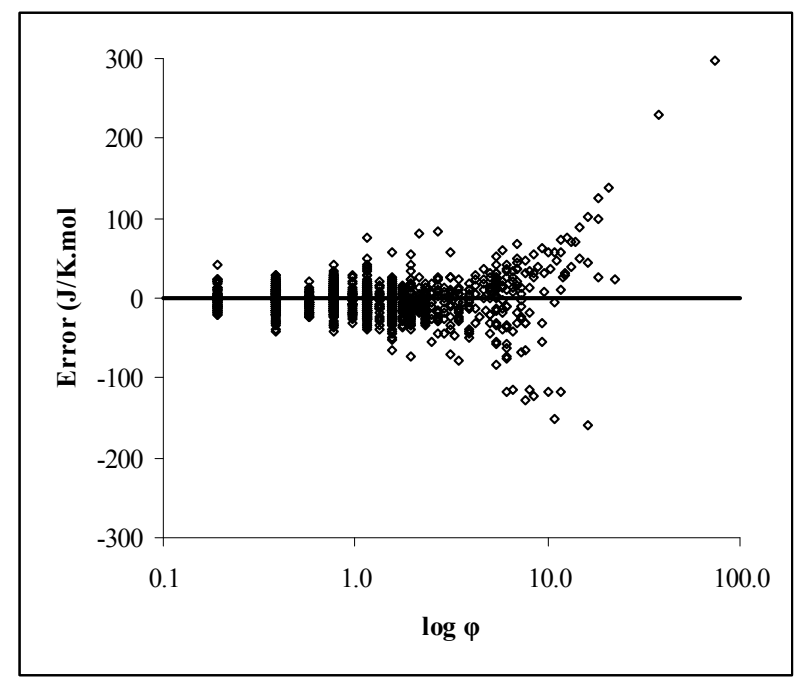

Figure3. 4. Error distribution as a function of the logarithm of the molecular flexibility number $(\varphi)$. Note that the $x$-axis is on a logarithmic scale. 
The effect of the large errors in entropy prediction for very long chain flexible molecules on the overall absolute average error can be seen in Table 3.2. The average absolute error increases as longer chain compounds are included in the dataset. It is evident that equation 3.3 gives better estimations of entropy of melting for compounds with lower flexibility counts.

Table 3.2. Average absolute errors in entropy of melting prediction at different flexibility count (Ф)

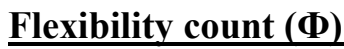
Number of compounds Average absolute $\operatorname{Error}(\mathbf{J} / \mathbf{K} . \mathbf{m o l})$

\begin{tabular}{lrc} 
Below 5 & 1380 & 11.8 \\
$5-10$ & 123 & 18.4 \\
$10-15$ & 72 & 21.0 \\
Above 15 & 88 & 54.5 \\
\hline
\end{tabular}

Table 3.3 gives a frequency distribution of the average absolute errors in the prediction of total entropy of melting for 1663 compounds. More than $77 \%$ of the compounds have average errors less than $20 \mathrm{~J} / \mathrm{K} . \mathrm{mol}$.

Table 3.3. Frequency distribution of the average absolute errors in entropy of melting prediction

\begin{tabular}{lcc} 
Average absolute & Number of & Cumulative \\
\hline Error (J/K.mol) & compounds & \%total \\
\hline Below 20 & 1286 & 77 \\
$20-40$ & 297 & 95 \\
$40-60$ & 43 & 98 \\
Above 60 & 37 & 100 \\
\hline
\end{tabular}


Two examples for predicting entropies of melting using the proposed model are shown in Appendix A. A complete list of experimental and calculated entropies of melting for the 1663 compounds is given in Appendix C. Also listed are the rotational symmetry number $(\sigma)$ and flexibility count $(\Phi)$ for each compound. All compounds are listed in alphanumeric order.

\section{SUMMARY}

The semi-empirical equation of Dannenfelser and Yalkowsky (equation 3.3) is validated on a large database of pharmaceutically and environmentally relevant organic compounds. This equation predicts the total entropies of melting for over 1600 organic compounds with an average absolute error of $15.0 \mathrm{~J} / \mathrm{K} . \mathrm{mol}$. It is a reasonable estimate considering that no coefficients were generated from experimental data for a training set. 


\section{CHAPTER 4: MELTING POINTS}

\section{INTRODUCTION}

Melting Point is one of the most widely reported physical properties for organic compounds. Despite the availability of vast amounts of experimental melting point data, there is no general relationship relating melting point directly to chemical structure. A simple method that can estimate melting points directly from chemical structure would be a valuable tool in pharmaceutical and environmental science. Such a method would assist in the evaluation and design of new drug derivatives and chemicals that would be within a specified range of melting point, solubility, and vapor pressure.

A number of attempts have been made to correlate melting points to chemical structure. Broadly, these methods fall into three categories: additive group contributions, non-group QSPR methods, and the enthalpy/entropy combinations.

Group contribution methods are the simplest of all methods but they are associated with greater errors. One of the primary reasons for these larger errors is their inability to

distinguish between isomers. Some of the significant group contribution methods for estimation of melting points were developed by Joback et al. (1987), Constantinou et al. (1994) and Marrero et al. (2001). Joback et al. developed a first-order group contribution method with 40 groups for organic compounds containing halogens, oxygen, nitrogen and sulfur. Constantinou et al. came up with a two-level group contribution scheme consisting of 63 first-order groups and 40 second-order groups. While the first-order 
groups accounted for the groups similar to those of Joback et al., the second-order groups helped distinguish between isomers and accounted for some proximity factors. Inclusion of second-order groups provides greater accuracy but at the same time, the method is time consuming and difficult to use. Marrero et al. updated this model further to include third-order groups that could account for more complex heterocyclic and large polyfunctional alicyclic compounds. This method has 165 first-order groups, 115 secondorder groups and 64 third-order groups. This method is advantageous in terms of its greater accuracy and wide applicability. However the large number of groups makes it cumbersome.

A number of QSPR methods are available for estimation of melting points. These methods are based on specific families of compounds, and their application is limited to these specific families. Alkanes (Charton et al. 1994, Needham et al. 1988), aldehydes, amines, ketones (Katritzky et al. 1993, Dearden, 1991) and benzenes (Katritzky et al. 1997) are some of the families for which QSPR models have been developed. According to a recent review by Katritzky et al. (2003), the current QSPR methods range in accuracy from very good (standard deviation $=15.1 \mathrm{~K}$ for $\mathrm{n}$-alkanes) to similar accuracy as group contributions (standard deviation $=36.1 \mathrm{~K}$ for pyridines). The major disadvantage of QSPR methods is their limited application to the specific class of compounds they are based on.

Melting points can also be estimated from the ratio of the enthalpy and entropy of melting. Enthalpy of melting is estimated by group contributions whereas the entropy of 
melting is correlated from QSPR descriptors for symmetry and flexibility. Such correlations have been developed for alkanes (Tsakanikas et al. 1988), aliphatics (Zhao et al. 1999, Krzyzaniak et al. 1995) and aromatic compounds (Simamora et al. 1993).

In addition to the above mentioned methods, Chickos et al. (2001) developed a parabolic model for estimating the melting points of homologous series. In yet another method developed by Yalkowsky et al., the melting points were estimated from the boiling points of organic compounds. The average absolute errors (AAE), number of compounds and compound categories for some of the methods discussed above are listed in Table 4.1.

Table 4.1. Average Absolute Errors in general melting point estimation methods

\begin{tabular}{lccl} 
Method & Number of Compounds & AAE & Compound Class \\
\hline Tsakanikas et al. & 72 & 15.7 & Alkanes \\
Simamora et al. & 123 & 47.6 & Rigid Aromatic \\
Krzyzaniak et al. & 497 & 30.3 & Non H-bonding Aliphatic \\
Zhao et al. & 794 & 39.9 & Aliphatic \\
Yalkowsky et al. & 918 & 31.7 & from Boiling Point \\
Constantinou et al. & 1007 & 34.6 & Organic \\
Joback et al. & 1117 & 43.0 & Organic \\
Marrero et al. & 1170 & 32.4 & Organic \\
\hline
\end{tabular}

Selection of a melting point estimation method is dependent upon the level of desired accuracy and applicability. QSPR models have greater accuracy in their particular class of compounds but their extrapolation to compounds outside the training set leads to very poor correlations. Group contribution schemes fail to distinguish between isomers and 
result in greater errors. The more general enthalpy/entropy combination approaches have wider applicability and are more valuable in cases where compounds do not fit within well-defined families or belong to families for which QSPR methods do not exist.

This chapter describes our melting point estimation model that is based on the combination of enthalpy and entropy using a large dataset of over 2200 organic compounds.

\section{DATA}

The experimental data for melting points used in this study is the same as described in Chapter 2.

\section{METHODS}

The melting point denotes the temperature at which a crystalline solid is transformed into a liquid. At the melting point, the free energy of transition is equal to zero and the thermodynamic expression for melting point is given by

$$
\mathrm{T}_{\mathrm{m}}=\frac{\Delta \mathrm{H}_{\mathrm{m}}}{\Delta \mathrm{S}_{\mathrm{m}}}
$$

Where

$\mathrm{T}_{\mathrm{m}}=$ Melting point $(\mathrm{K})$

$\Delta \mathrm{H}_{\mathrm{m}}=$ total phase change enthalpy of melting $(\mathrm{kJ} / \mathrm{mol})$

$\Delta \mathrm{S}_{\mathrm{m}}=$ total phase change entropy of melting $(\mathrm{J} / \mathrm{K} . \mathrm{mol})$ 
The enthalpy and entropy of melting are calculated as described in Chapters 2 and 3 respectively.

\section{RESULTS AND DISCUSSION}

As described in equation 4.1, the melting points are estimated from the ratio of the enthalpy and entropy of melting. The average absolute error is $30.1^{\circ}$ for melting points ranging from $85.5 \mathrm{~K}$ to $710.5 \mathrm{~K}$.

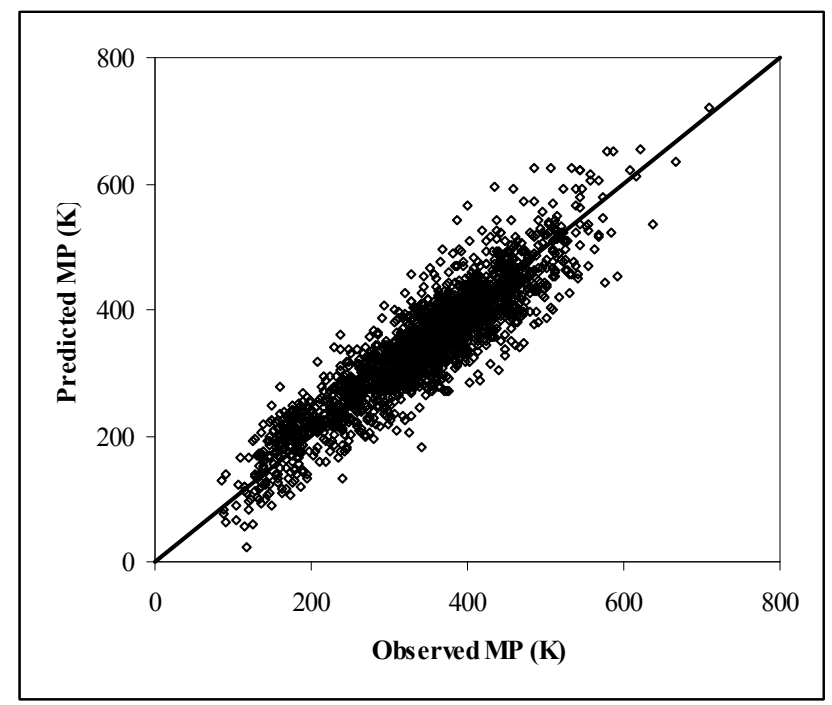

Figure4.1. Observed versus predicted melting points for 2230 organic compounds

Figure 4.1 shows a plot of predicted versus observed melting points for the entire data set. The narrow distribution of values along the line of identity suggests an accurate prediction using the proposed model. Most of the values are overlapping because of the large number of observations in the study. 
Figure 4.2 shows the error distribution obtained in correlating the experimental and predicted melting points. The distribution is fairly symmetrical with few outliers.

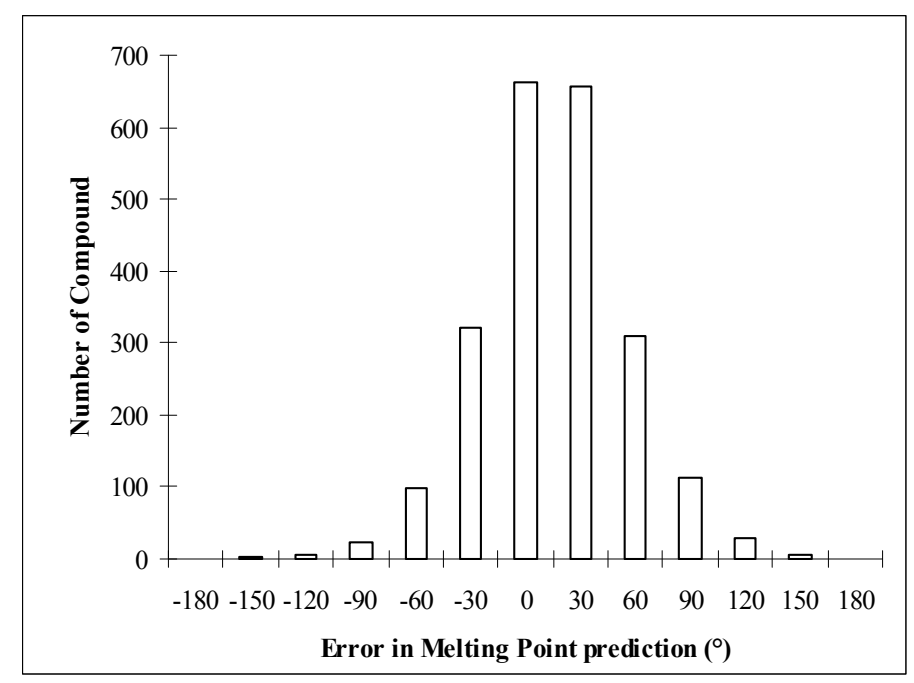

Figure4.2. Error distribution in melting point prediction using the proposed model

Table 4.2 gives a frequency distribution of the average absolute errors in melting point prediction. Only $2 \%$ of the compounds have average absolute errors greater than $100^{\circ}$. It is to be noted that a $100^{\circ}$ error corresponds to a ten-fold error in calculated solubility by Yalkowsky's General Solubility Equation (Ran et al., 2001).

Table 4.2. Frequency distribution of the average absolute errors in melting point prediction

\begin{tabular}{lcc} 
Average absolute & Number of & Cumulative \\
\hline Error & compounds & \% total \\
\hline Below $30^{\circ}$ & 1320 & 59 \\
$30^{\circ}-60^{\circ}$ & 630 & 87 \\
$60^{\circ}-90^{\circ}$ & 211 & 97 \\
Above $90^{\circ}$ & 69 & 100 \\
\hline
\end{tabular}


A three-fold cross validation was performed on the database to validate the prediction ability of this method. For each validation, approximately $1 / 10^{\text {th }}$ of the data was selected at random (RAND function, Microsoft Excel 2000) and used as the test set. The remaining data were used as the training set to generate the group contribution values. Average absolute errors were calculated for each pair of test and training sets. The results for cross validation are listed in Table 4.3, where $\mathrm{N}$ represents the number of compounds in each set. The similarity of the absolute errors for the test and training sets and the overall absolute error strongly validates the predictability of this method.

Table 4.3. Cross-validation of proposed method

Cross Validation Training Set

Test set

\begin{tabular}{lllll} 
& AAE & N & AAE & N \\
\hline Round 1 & $29.9^{\circ}$ & 2007 & $33.1^{\circ}$ & 222 \\
Round 2 & $29.7^{\circ}$ & 2007 & $37.2^{\circ}$ & 218 \\
Round 3 & $30.0^{\circ}$ & 2007 & $33.8^{\circ}$ & 216 \\
\hline
\end{tabular}

$\mathrm{AAE}=$ Average absolute error in melting point prediction, $\mathrm{N}=$ number of compounds

The accuracy of melting point predictions obtained with the proposed model is attributed mainly to the separate calculation of the enthalpy and entropy of melting. This is a distinct advantage over group contribution methods that are incapable of accounting for the effect of symmetry and flexibility. The chemical diversity of over twenty-two hundred compounds studied illustrates the fact that this model has wide applicability. It gives melting point predictions ranging over nearly nine orders of magnitude with an average absolute error of $30^{\circ}$. 
In addition, we are able to predict melting points of complex structures including many drugs, steroids, herbicides and pesticides. The identification and inclusion of large, complex molecular fragments using the isolating carbon concept is a useful tool for calculating the enthalpy of melting and subsequently the melting points of these complex structures.

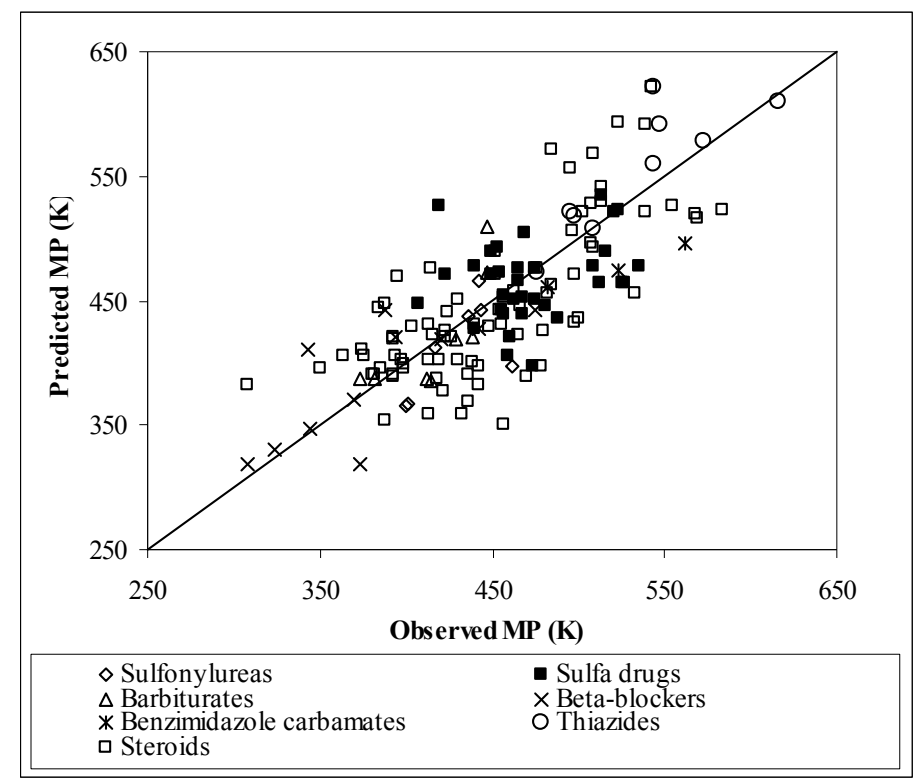

Figure4.3. Predicted versus observed melting points for 152 drugs (including steroids) in the database 


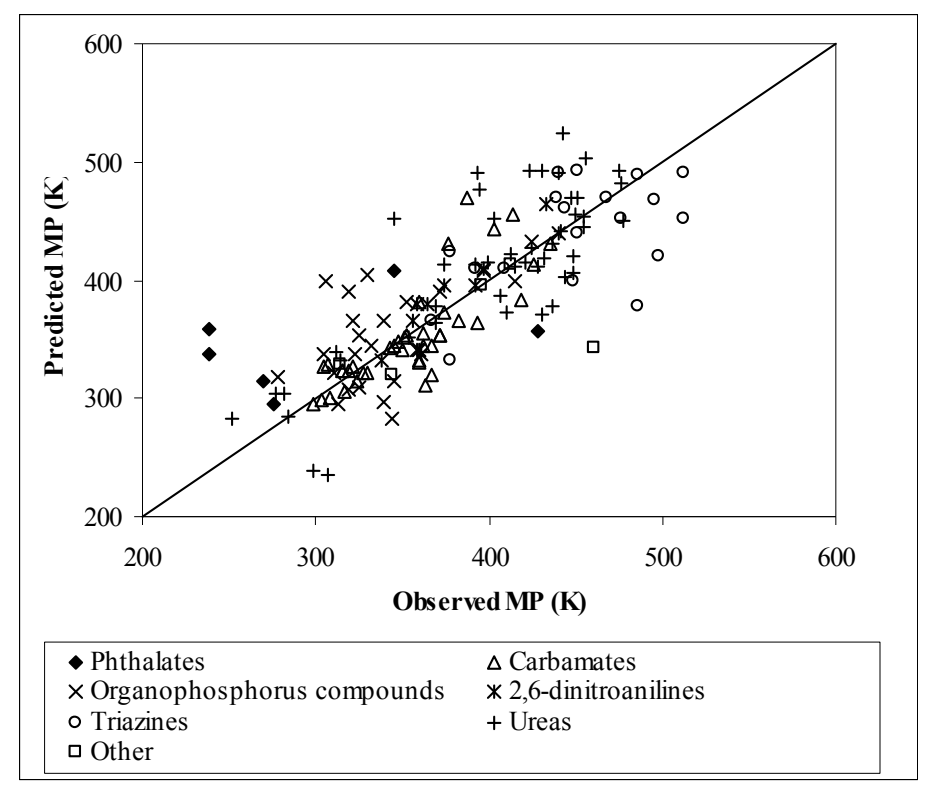

Figure4.4. Predicted versus observed melting points for 149 environmental compounds included in the database

Figure 4.3 and 4.4 show the observed versus predicted melting points of 152 drugs (including steroids) and 149 environmental compounds respectively. In both cases, the distribution of values along the line of identity is narrow. The melting points of about $75 \%$ of the drugs and $85 \%$ of the environmental compounds are off by less than $50^{\circ}$. The proximity factors for intramolecular hydrogen bonding, geminal halogen atoms and $-\mathrm{OH}$ and $-\mathrm{NH}_{2}$ groups on secondary and tertiary carbons contribute to accurate enthalpy of melting and melting point predictions. For example, as seen in Figure 4.5, a large improvement is observed in the melting point prediction of dinitramine when proximity factors for intramolecular hydrogen bond and geminal halogens are included in the enthalpy calculation. 


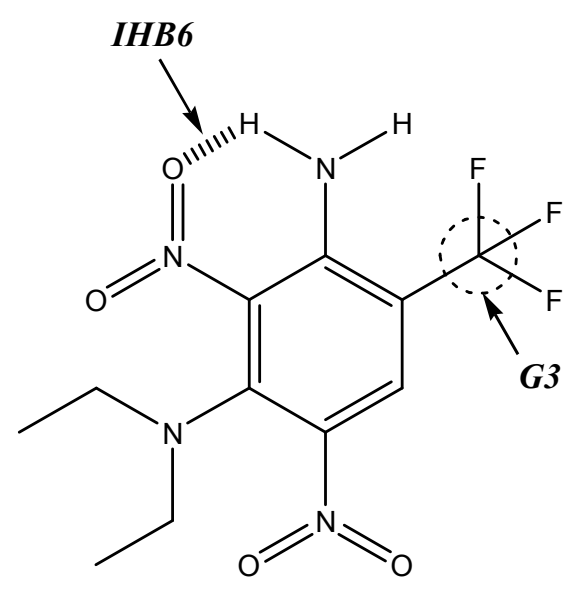

Observed Melting Point: $372.1 \mathrm{~K}$

Predicted Melting Point (no proximity factors): $459.1 \mathrm{~K}$

Predicted Melting Point (with proximity factors): $390.0 \mathrm{~K}$

Figure4.5. Proximity factors for intramolecular hydrogen bonding and geminal fluorine atoms in dinitramine

Two examples for predicting melting points using the proposed model are shown in Appendix A. A complete list of the 2230 organic compounds along with their observed and predicted melting points is provided in Appendix D. The compounds are arranged in alphanumeric order.

The proposed model is in agreement with the principle of convergence temperature. When the proposed model was applied to a dataset of 40 linear alkanes ranging from $\mathrm{C} 2$ C190, a convergence similar to the experimental melting points was observed as seen in Figure 4.6. 


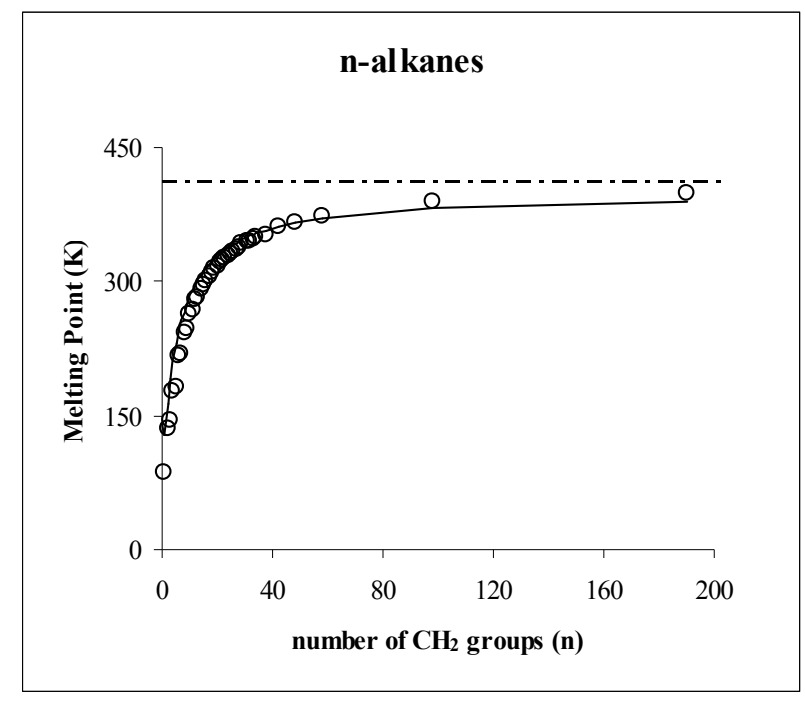

Figure 4.6. Experimental melting points of n-alkanes (C2-C190) (०). The dotted line represents predicted values. The dashed line represents the convergence temperature at $410.0 \mathrm{~K}$.

Addition of a methylene group to a linear hydrocarbon increases its van der Waals interaction energy and thus its enthalpy of melting by a constant value. This would suggest a linear increase in melting-points with an increase in chain length. However, with an increase in the number of methylene groups, the flexibility term becomes an important factor and it adds to the entropy of melting by a constant value. If the enthalpy and entropy of melting are expressed as linear functions of chain length (n), melting point equation can be written as

$$
\mathrm{T}_{\mathrm{m}}=\frac{\Delta \mathrm{H}_{\mathrm{m}}}{\Delta \mathrm{S}_{\mathrm{m}}}=\frac{\mathrm{a}+\mathrm{bn}}{\mathrm{c}+\mathrm{dn}}
$$


Similar equations were derived by Broadhurst (1963) and Flory and Vrij (1963). Chickos and Nichols (2001) developed a hyperbolic function of chain-length to estimate the melting temperatures of homologous series. According to the above equation, as $n \rightarrow \infty$, $T_{m}$ approaches a limiting value of $b / d$, also known as the convergence temperature. For alkanes, this value has been experimentally determined to be $410 \mathrm{~K}$ (dashed line in Figure 4.6). Based on an enthalpic group contribution value of $2.95 \mathrm{~kJ} / \mathrm{mol}$ and an entropy value of $7.382 \mathrm{JK}^{-1} \mathrm{~mol}^{-1}$ for a methylene group, the proposed method estimates the convergence temperature for alkanes to be $399.6 \mathrm{~K}$.

\section{SUMMARY}

Melting points are predicted for a chemically diverse database of 2230 organic compounds with an average absolute error of $30.1^{\circ}$. The proposed model provides an accurate and widely applicable tool for predicting melting points directly from molecular structure. 


\section{CHAPTER 5: COMPARISON OF TWO METHODS FOR PREDICTING MELTING POINTS}

\section{INTRODUCTION}

In this chapter, the melting point predictions of UPPER as described in this dissertation are compared to those of MPBPWIN for 2230 organic compounds. MPBPWIN is a program available through the Environmental Protection Agency. It predicts melting points using two different group contribution methods.

\section{DATA}

The dataset used in this study is the same as used in Chapters 2, 3 and 4.

\section{METHODS}

In UPPER, melting points are calculated using the following relationship:

$$
\mathrm{T}_{\mathrm{m}}=\frac{\Delta \mathrm{H}_{\mathrm{m}}}{\Delta \mathrm{S}_{\mathrm{m}}}
$$

Where

$\mathrm{T}_{\mathrm{m}}=$ Melting point $(\mathrm{K})$

$\Delta \mathrm{H}_{\mathrm{m}}=$ total phase change enthalpy of melting $(\mathrm{kJ} / \mathrm{mol})$

$\Delta \mathrm{S}_{\mathrm{m}}=$ total phase change entropy of melting $(\mathrm{J} / \mathrm{K} \cdot \mathrm{mol})$ 
The total phase change enthalpy and entropy of melting were calculated as described in Chapters 2 and 3 respectively.

MPBPWIN estimates melting points using two different group contribution methods. The first method is based on the group contribution scheme developed by Joback (1984) and Reid et al. (1987). The second method was suggested by Lyman (1985) and was originally developed by Gold et al. (1969). In this method, the melting points are calculated from the boiling points using the following relationship:

$$
\mathrm{T}_{\mathrm{m}}=0.5839 \mathrm{~T}_{\mathrm{b}}
$$

The boiling points $(\mathrm{Tb})$ used in this equation are obtained by group contributions. In addition, the MPBPWIN program also contains correction factors for specific structures. In order to evaluate the two models, average absolute errors (AAE) and root mean square errors (RMSE) were calculated for each method.

AAE was determined by

$$
\begin{aligned}
& \mathrm{AAE}=\frac{\sum\left|\mathrm{T}_{\mathrm{m}, \mathrm{obs}}-\mathrm{T}_{\mathrm{m}, \text { pred }}\right|}{\mathrm{N}} \\
& \mathrm{N}=\text { number of compounds }
\end{aligned}
$$

RMSE was determined by 


$$
\text { RMSE }=\sqrt{\frac{\sum\left(\mathrm{T}_{\mathrm{m}, \mathrm{obs}}-\mathrm{T}_{\mathrm{m}, \text { pred }}\right)^{2}}{\mathrm{~N}}}
$$

$\mathrm{N}=$ number of compounds

\section{RESULTS AND DISCUSSION}

Plots of observed versus predicted melting points for the 2230 organic compounds by each method are shown in Figures 5.1 and 5.2. As seen in the two figures, UPPER based melting point estimation model has an R-squared value of 0.830 whereas MPBPWIN has an R-squared value of 0.645 .

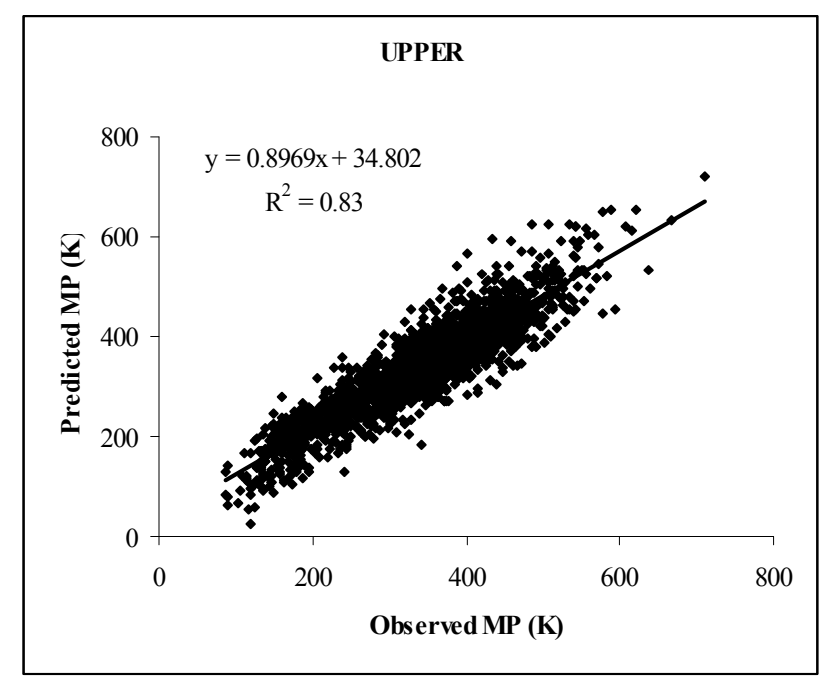

Figure 5.1. Observed versus predicted melting points (using UPPER) for 2230 compounds 


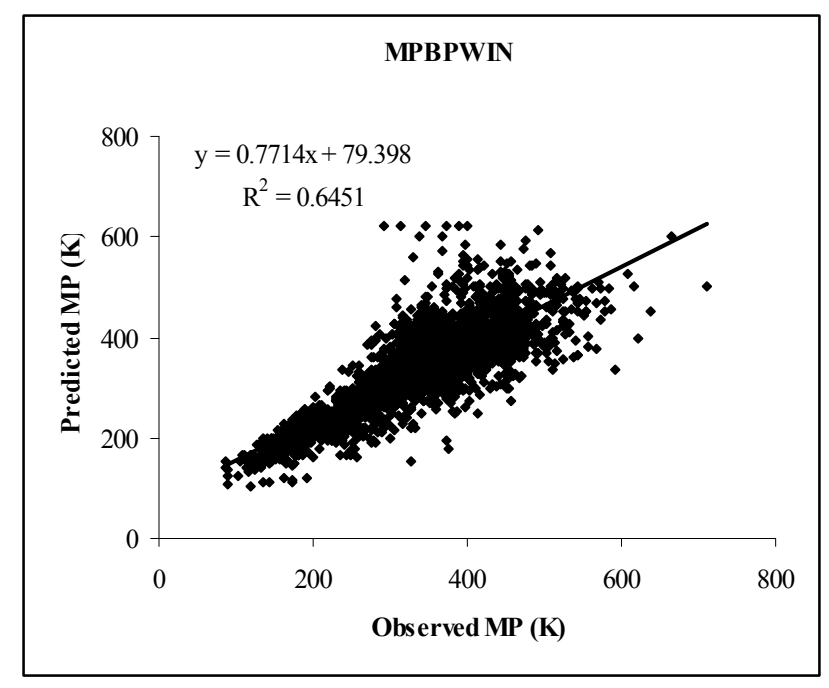

Figure 5.2. Observed versus predicted melting points (using MPBPWIN) for 2230 compounds

The AAE for UPPER is $30.1^{\circ}$ as compared to $44.5^{\circ}$ for MPBPWIN. Also the RMSE for UPPER $\left(39.7^{\circ}\right)$ is considerably lower than that obtained using MPBPWIN $\left(58.4^{\circ}\right)$. These values clearly indicate that the UPPER model provides a better prediction of melting points as compared to MPBPWIN for a large, diverse set. The greater accuracy of UPPER melting point predictions is based on the fact that it accounts for both the additive and non-additive aspects of the melting process. MPBPWIN, which is based solely on additive group contributions, does not account for the geometric factors involved in melting. Group contribution alone cannot distinguish between the melting points of isomers that have, in instances, melting points differing by over $100^{\circ}$. In contrast, UPPER includes the entropy of melting in its melting point prediction. The entropy of melting term consists of the rotational symmetry number that differentiates between isomers and the flexibility number that accounts for the conformational changes upon melting, especially for long, flexible molecules. 


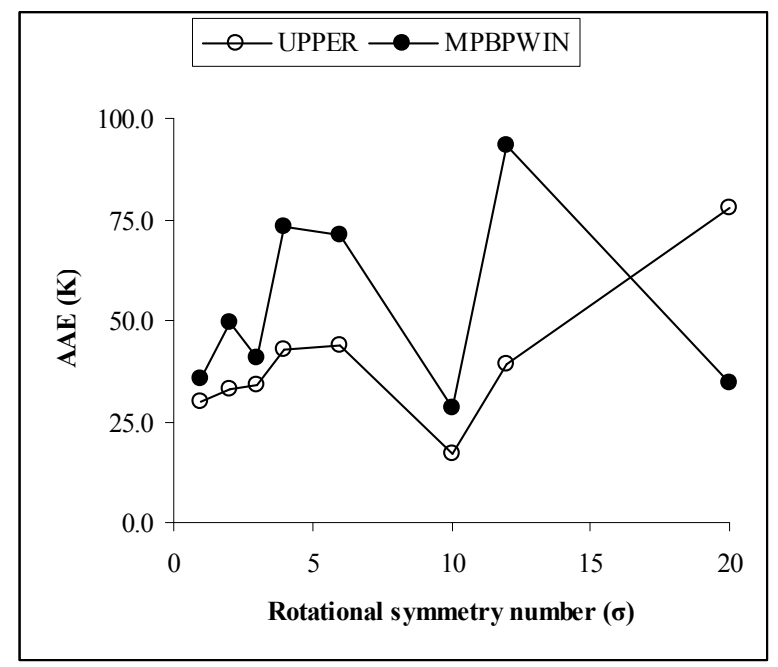

Figure 5.3. AAE in melting point prediction of symmetrical molecules using the two models

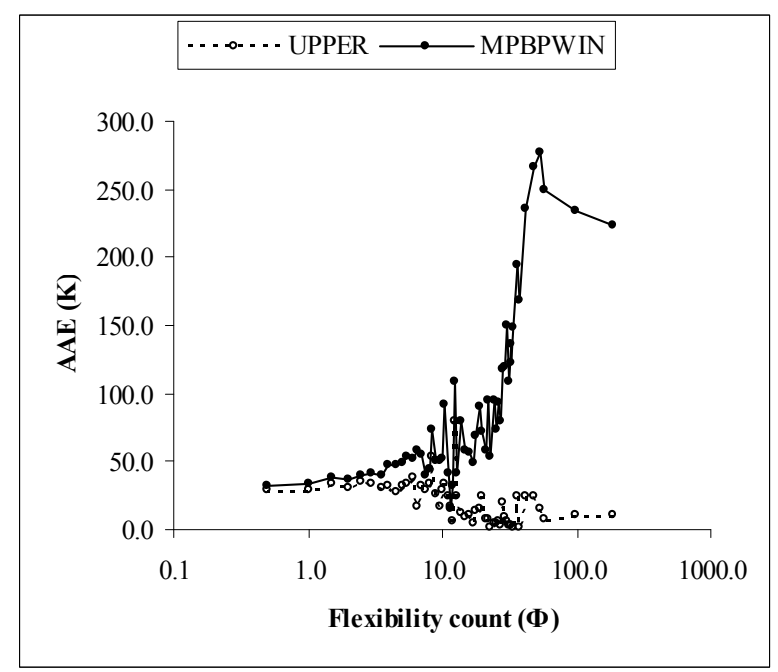

Figure 5.4. AAE in melting point predictions of flexible molecules using the two models. Note that the $x$-axis is on a logarithmic scale

Figures 5.3 and 5.4 are plots of AAE for each method versus the rotational symmetry number and the flexibility number respectively. To reduce the number of data points, the AAE for compounds having same symmetry number or flexibility number are averaged. 
It is evident from both figures that UPPER consistently provides more accurate predictions of melting points for a diverse set of compounds, ranging from highly symmetrical rigid molecules to long, flexible molecules.

In addition, the enthalpy calculation in UPPER is based on a group contribution scheme. This scheme is based on the isolating carbon rule and is of great utility in identifying complex molecular fragments often encountered in chemical structures of drugs and pesticides. The scheme also contains proximity factors like intramolecular hydrogen bonding and geminal halogen atoms. A combination of this additive group contribution scheme and the non-additive entropic contributions results in a melting point estimation model that is reasonably accurate and widely applicable.

\section{SUMMARY}

Two widely applicable models for predicting melting points directly from chemical structure are compared. The average absolute error (AAE) and root mean square error (RMSE) in melting point prediction using UPPER are $30.1^{\circ}$ and $39.7^{\circ}$, while MPBPWIN gives an AAE of $44.5^{\circ}$ and RMSE of $58.4^{\circ}$. UPPER provides more accurate melting point predictions because it accounts for both the additive enthalpic and non-additive entropic contributions to melting. 
APPENDIX A. Examples of predicting enthalpy of melting $\left(\Delta \mathrm{H}_{\mathrm{m}}\right)$, entropy of melting $\left(\Delta \mathrm{S}_{\mathrm{m}}\right)$ and melting point $\left(\mathrm{T}_{\mathrm{m}}\right)$ using the developed model.

(a) Butanoic Acid

\section{$\mathrm{CH}_{3}-\mathrm{CH}_{2}-\mathrm{CH}_{2}-\mathrm{C}(=\mathrm{O})(\mathrm{OH})$}

Parameters: Observed $\mathrm{T}_{\mathrm{m}}=264.7 \mathrm{~K}, \sigma=1, \Phi=2.435^{(1.5)}$

$$
\begin{aligned}
& \begin{aligned}
\Delta \mathrm{H}_{\mathrm{m}} & =\Sigma \mathrm{n}_{\mathrm{i}} \mathrm{m}_{\mathrm{i}}=1(\mathrm{XCH} 3)+1(\mathrm{XCH} 2)+1(\mathrm{YCH} 2)+1(\mathrm{XCOOH}) \\
& =1(1.76)+1(2.95)+1(2.10)+1(9.91)=16.72 \mathrm{~kJ} / \mathrm{mol}
\end{aligned} \\
& \begin{aligned}
\Delta \mathrm{S}_{\mathrm{m}} & =50-\mathrm{R} \ln \sigma+\mathrm{R} \ln \phi=50-8.31 \ln (1)+8.31 \ln \left[2.435^{(1.5)}\right]=61.1 \mathrm{~J} / \mathrm{K} . \mathrm{mol} \\
\mathrm{T}_{\mathrm{m}}= & \Delta \mathrm{H}_{\mathrm{m}} / \Delta \mathrm{S}_{\mathrm{m}}=16720 / 61.1=273.6 \mathrm{~K}
\end{aligned}
\end{aligned}
$$

(b) Benzoic acid

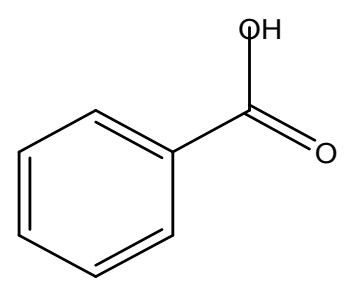

Parameters: Observed $\mathrm{T}_{\mathrm{m}}=395.5 \mathrm{~K}, \sigma=2, \Phi=2.435^{(0)}$

$$
\Delta \mathrm{H}_{\mathrm{m}}=\Sigma \mathrm{n}_{\mathrm{i}} \mathrm{m}_{\mathrm{i}}=1(\mathrm{Car})+5(\mathrm{CHar})+1(\mathrm{YCOOH})
$$


$=1(-0.86)+5(1.59)+1(12.15)=19.24 \mathrm{~kJ} / \mathrm{mol}$

$$
\begin{aligned}
& \Delta \mathrm{S}_{\mathrm{m}}=50-\mathrm{R} \ln \sigma+\mathrm{R} \ln \phi=50-8.31 \ln (2)+8.31 \ln \left[2.435^{(0)}\right]=44.2 \mathrm{~J} / \mathrm{K} . \mathrm{mol} \\
& \mathrm{T}_{\mathrm{m}}=\Delta \mathrm{H}_{\mathrm{m}} / \Delta \mathrm{S}_{\mathrm{m}}=19240 / 44.2=435.3 \mathrm{~K}
\end{aligned}
$$




\title{
APPENDIX B. Experimental and predicted enthalpy of melting $\left(\Delta \mathrm{H}_{\mathrm{m}}\right)$ for
}

\author{
1663 organic compounds.
}

Name

$\Delta \mathbf{H}_{\mathrm{m}}(\mathrm{kJ} / \mathrm{mol})$

(+)2-butanol

experimental predicted

(+)a-(3-benzoylphenyl)propionic acid

6.00

28.23

11.16

(2,4,5-trichlorophenoxy)acetic acid

(2,4-dichlorophenoxy)acetic acid

(4-chloro-2-methylphenoxy)acetic acid

(4-chloro-o-tolyloxy)acetic acid

(d) 1,2-dibromoacenaphthene

$38.00 \quad 26.72$

(d) 1,2-dichloroacenaphthene

$35.33 \quad 25.69$

$29.98 \quad 25.46$

(d) 1,2-diphenyl-1,2-dihydroxyethane

$29.98 \quad 25.46$

$26.36 \quad 19.41$

(d) 2-(1-naphthoxy)propionamide

(D) 2-(2-chloro-3-methylphenoxy)propionic acid

21.34

17.77

$34.31 \quad 25.83$

(d) 2-(m-chlorophenoxy)propanoic acid

$38.07 \quad 30.52$

(d) 2-(o-chlorophenoxy)propanoic acid

22.18

25.79

$29.71 \quad 25.00$

(d) 2-(p-bromophenoxy)propanoic acid

$26.78 \quad 25.00$

(d) 2-(p-methoxyphenyl)propiophenone

$27.61 \quad 25.99$

(d) 2-(p-nitrophenoxy)propanoic acid

$21.76 \quad 22.87$

(d) 2,3-dibromo-1,4-butanediol

(d) 2-phenoxypropionic acid

$20.92 \quad 27.41$

(d) 3-(m-bromophenyl)-3-hydroxypropanoic acid

33.89

24.72

22.59

23.97

(d) 3-(m-chlorophenyl)-3-hydroxypropanoic acid

23.85

26.94

$28.03 \quad 25.95$

(d) 3-(m-fluorophenyl)-3-hydroxypropanoic acid

24.27

24.94

(d) 3-(o-fluorophenyl)-3-hydroxypropanoic acid

22.59

24.94

(d) 3-(p-bromophenyl)-3-hydroxypropanoic acid

35.56

26.94

(d) 3-(p-chlorophenyl)-3-hydroxypropanoic acid

28.03

25.95

(d) 3-(p-fluorophenyl)-3-hydroxypropanoic acid

30.96

24.94

(d) 3-hydroxy-3-phenylbutyric acid

22.59

25.53

(d) 3-hydroxy-3-phenylvaleric acid

30.96

28.48

(d) 3-phenyl-3-hydroxy-2,2-dimethylpropanoic acid

39.75

26.78

(d) 3-phenyl-3-hydroxypropanoic acid

32.64

24.92

(d) dimethyl diacetyltartrate

29.29

27.49

(d) dimethyl tartrate

17.36

23.06

(d) malic acid

23.01

27.55

(d) mandelic acid

26.36

21.85

(d) methylenebisthiopropionic acid

22.59

33.26

(d) m-fluoromandelic acid

24.27

21.88

(d) o-chloromandelic acid

24.69

22.88

(d) o-fluoromandelic acid

20.92

21.88

(d) p-chloromandelic acid

23.01

22.88

(d) p-fluoromandelic acid

30.54

21.88

(dl) 1,2-dibromoacenaphthene

25.10

19.41

(dl) 1,2-dichloroacenaphthene

20.50

17.77

(dl) 1,2-diphenyl-1,2-dihydroxyethane

31.38

25.83

(dl) 2-(1-naphthoxy)propionamide

37.66

30.52

(dl) 2-(2-chloro-3-methylphenoxy)propionic acid

30.54

25.79

(dl) 2-(m-chlorophenoxy)propanoic acid

33.05

25.00 
(dl) 2-(o-chlorophenoxy)propanoic acid

32.22

25.00

(dl) 2-(p-bromophenoxy)propanoic acid

25.99

(dl) 2-(p-methoxyphenyl)propiophenone

31.80

22.87

(dl) 2-(p-nitrophenoxy)propanoic acid

26.36

27.41

(dl) 2,3-dibromo-1,4-butanediol

32.22

24.72

(dl) 2-phenoxypropionic acid

23.97

(dl) 3-(m-bromophenyl)-3-hydroxypropanoic acid

33.05

26.94

(dl) 3-(m-chlorophenyl)-3-hydroxypropanoic acid

26.78

25.95

(dl) 3-(m-fluorophenyl)-3-hydroxypropanoic acid

23.85

24.94

(dl) 3-(o-fluorophenyl)-3-hydroxypropanoic acid

20.50

24.94

(dl) 3-(p-bromophenyl)-3-hydroxypropanoic acid

27.20

26.94

(dl) 3-(p-chlorophenyl)-3-hydroxypropanoic acid

28.87

25.95

(dl) 3-(p-fluorophenyl)-3-hydroxypropanoic acid

29.71

24.94

(dl) 3-hydroxy-3-phenylbutyric acid

27.61

25.53

(dl) 3-hydroxy-3-phenylvaleric acid

19.66

28.48

(DL) 3-methylnonane

(dl) 3-phenyl-3-hydroxy-2,2-dimethylpropanoic acid

35.15

22.51

(dl) 3-phenyl-3-hydroxypropanoic acid

18.70

26.78

(DL) 4-methylnonane

37.24

24.92

$15.19 \quad 22.51$

(dl) dimethyl diacetyltartrate

27.49

(dl) dimethyl tartrate

25.94

23.06

(dl) malic acid I

26.94

(dl) malic acid II

33.52

27.55

(dl) mandelic acid

30.17

27.55

(dl) menthol

25.52

21.85

$10.25 \quad 16.71$

(dl) methylenebisthiopropionic acid

39.33

33.26

(dl) m-fluoromandelic acid

24.69

21.88

(dl) o-chloromandelic acid

20.08

22.88

(dl) o-fluoromandelic acid

30.12

21.88

(dl) p-chloromandelic acid

27.20

(dl) p-fluoromandelic acid

29.29

(l) menthol

11.88

31.46

[(benzoylamino)oxy] acetic acid

22.87

1-(4-chlorophenoxy)-3,3-dimethyl-(1H,1,2,4-triazol-1-yl)-2-butanone

1-(methylamino)-9,10-anthracenedione

1 -(o-chlorophenyl)thiourea

22.29

26.28

1,1-(2,2,2-trichloroethylidene)bis(4-chlorobenzene)

27.48

1,1 '-(2,2,2-trichloroethylidene-bis(4-methoxy)benzene

23.34

22.88

21.88

16.71

31.62

28.82

27.42

21.40

24.04

29.69

22.91

1,1'-(2,2-dichloroethylidenebis(4-chlorobenzene)

27.31

22.15

1,1-(di-p-chlorophenyl)-2-nitropropane

21.39

23.23

12.69

17.85

1,1,1,3-tetrachloropropane

1,1,1-trichloro-3,3,3-trifluoropropane

14.07

13.70

10.13

9.87

1,1,1-trifluoro-3,3-dichloropropane

10.33

11.81

1,1,1-trifluoro-3-chloropropane

9.80

12.40

1,1,1-trifluoroethane

$6.19 \quad 4.41$

1,1,1-trifluoro-n-[2-methyl-4-(phenylsulphonyl)phenyl]

methane sulfonamide

31.79

27.67

1,1,1-trinitroethane

16.32

13.33

1,1,2,2-tetrachlorodifluoroethane

5.52

12.57

1,1,2,2-tetrachloroethane

9.72

12.43

1,1,2-tribromoethane

9.11

10.11 
1,1,2-trichloroethane

$11.38 \quad 13.02$

1,1,2-trifluoro-1,2,2-trichloroethane

$\begin{array}{rr}4.86 & 10.75 \\ 20.55 & 25.67\end{array}$

1,1,3,3-tetraethylurea

1,1,3-trimethylurea

$14.30 \quad 19.70$

1,10-decanediol

$\begin{array}{ll}41.70 & 39.91\end{array}$

1,12-benzoperylene

$\begin{array}{ll}17.37 & 20.83 \\ 15.41 & 26.18\end{array}$

1,1-bis(4-chlorophenyl)-2-nitrobutane

1,1-dichloro-2,2-bis(4-chlorophenyl)ethylene

$\begin{array}{rr}23.55 & 16.88 \\ 7.87 & 7.98\end{array}$

1,1-dichloroethane

1,1-dichloroethene

$6.51 \quad 6.91$

1,1-dicyclohexyldodecane

44.35

53.91

1,1-diethylurea

20.37

26.85

1,1-difluoro-1-chloroethane

2.69

6.23

1,1-dimethyl-3-phenylurea

22.81

25.03

1,1-dimethylcyclohexane

$11.37 \quad 13.24$

1,1-dimethylcyclopentane

10.09

10.91

1,1-dimethylurea

1,1-diphenyldodecane

$1,2,3,4,5,6,7,8$-octahydroanthracene

29.11

20.96

$41.68 \quad 45.12$

$20.86 \quad 22.52$

$38.90 \quad 30.38$

$37.60 \quad 30.38$

1,2,3,4,5-pentahydroxypentane (Xylitol)

$37.40 \quad 30.38$

1,2,3,4-tetracarbomethoxybenzene

$40.40 \quad 30.98$

1,2,3,4-tetracarbomethoxynaphthalene

35.90

34.51

$17.00 \quad 13.65$

1,2,3,4-tetrachlorobenzene

10.93

9.62

$12.45 \quad 16.03$

1,2,3,4-tetrahydronaphthlene

11.81

17.08

1,2,3,4-tetrahydroquinoline

42.36

25.50

1,2,3,4-tetramethylbenzene

1,2,3,5-tetracarbomethoxybenzene

11.23

12.70

$32.60 \quad 30.98$

1,2,3,5-tetrachlorobenzene

19.00

13.65

$\begin{array}{ll}10.67 & 9.62\end{array}$

12.93

12.70

1,2,3,5-tetramethylbenzene

23.52

21.39

1,2,3-tribromopropane

1,2,3-tricarbomethoxybenzene

23.78

19.44

32.70

27.02

1,2,3-tricarbomethoxynaphthalene

23.70

29.15

1,2,3-trichlorobenzene

20.50

12.62

1,2,3-trihydroxybenzene

18.55

20.66

1,2,3-trihydroxypropane

18.28

21.06

1,2,3-trimethylbenzene

10.17

11.91

1,2,4,5,8,9-tribenzopyrene

28.80

27.54

1,2,4,5-tetrabromobenzene

28.22

17.60

$35.70 \quad 30.98$

1,2,4,5-tetracarbomethoxynaphthalene

$36.40 \quad 34.51$

1,2,4,5-tetrachloro-3-nitrobenzene

$19.46 \quad 17.09$

1,2,4,5-tetrachlorobenzene

$24.10 \quad 13.65$

1,2,4,5-tetrafluorobenzene

15.05

9.62

$20.88 \quad 12.70$

1,2,4,5-tetramethylbenzene

16.10

17.52

1,2,4-tricarbomethoxynaphthalene

32.10

29.15

1,2,4-trichloro-5-((4-chlorophenyl)sulfonyl)benzene

28.94

22.50 
1,2,4-trimethylbenzene

1,2,5,6-tetracarbomethoxynaphthalene

42.10

34.51

1,2,5-tricarbomethoxynaphthalene

25.50

29.15

1,2,6,7-tetracarbomethoxynaphthalene

34.20

34.51

1,2,6-tricarbomethoxynaphthalene

35.90

29.15

1,2,7,8-tetrahydroxyoctane

36.70

37.49

1,2,7-tricarbomethoxynaphthalene

36.10

29.15

1,2,8-tricarbomethoxynaphthalene

24.80

29.15

1,2:3,4-dibenzanthracene

25.82

23.65

1,2:3,4-dibenzopyrene

24.68

24.01

1,2:4,5-dibenzopyrene

1,2:5,6-dibenzanthracene

30.50

24.01

1,2-benzacenaphthene (fluoranthene)

31.16

23.65

$18.74 \quad 16.95$

1,2-benzanthracene

21.38

20.12

1,2-benzofluorene

22.20

19.04

1,2-benzopyrene

1,2-bromochlorobenzene

19.08

20.48

1,2-bromoiodobenzene

12.37

12.58

1,2-butadiene

14.42

14.53

1,2-chloronitrobenzene

6.95

4.33

$19.08 \quad 14.00$

1,2-diamino-2-methylpropane

18.90

15.35

1,2-diaminopropane

18.49

15.55

1,2-dibenzoylethane

39.58

8.30

1,2-dibromo-1,1-difluoroethane

12.61

1,2-dibromobenzene

13.14

25.13

12.91

13.57

1,2-dibromoethane

7.04

15.24

1,2-dibromotetrafluoroethane

16.95

10.57

1,2-dicarbomethoxybenzene

27.60

20.26

1,2-dicarbomethoxynaphthalene

12.93

1,2-dichlorobenzene

1,2-dichloroethane

1,2-dichloropropane

8.83

6.40

7.04

1,2-dichloro-tetrafluoroethane

20.00

23.79

11.59

13.60

11.94

1,2-dicyanobenzene

8.19

8.93

1,2-difluoro-2,2-dichloroethane

17.80

1,2-difluorobenzene

1,2-dihydro-6-neopentyl-2-oxonicotinic acid

11.05

19.33

1,2-dihydroxybenzene

22.01

14.01

1,2'-dinaphthylmethane

30.54

1,2-dinitrobenzene

22.84

1,2-diphenyl-2-(N-piperidinyl)-1-ethanone

33.93

25.40

7.56

1,2-pentadiene

30.31

29.37

1,3,5,5-tetranitro-1,3-diazacyclohexane

22.59

11.27

9.58

26.92

16.57

15.50

23.32

16.40

29.25

18.36

4.95

20.56

31.00

1,3,5,7-tetroxane

42.26

26.60

1,3,5-tri-a-naphthylbenzene

1,3,5-tricarbomethoxynaphthalene

1,3,5-trichloro-2,4,6-trifluorobenzene

25.90

35.70

19.83

29.15

12.69

18.20

12.62

1,3,5-trimethylbenzene

9.51

11.91

1,3,5-trinitro-1,3,5-triazacyclohexane

37.66

31.31 
1,3,5-trinitrobenzene

16.71

19.84

1,3,5-trinitroso-1,3,5-triazacyclohexane

21.98

28.49

1,3,5-trioxane

15.11

19.95

1,3,5-triphenylbenzene

33.40

25.11

1,3,6-trimethyluracil

21.20

18.74

1,3,7-tricarbomethoxynaphthalene

37.20

29.15

1,3,7-trichlorodibenzodioxin

$30.80 \quad 19.87$

1,3,8-tricarbomethoxynaphthalene

27.70

29.15

1,3-bromochlorobenzene

$12.29 \quad 12.58$

1,3-bromoiodobenzene

12.16

14.53

1,3-butadiene

7.98

7.94

1,3-cyclohexadiene

4.20

10.80

1,3-dibromobenzene

13.21

1,3-dibromopropane

14.64

13.57

1,3-dibutylurea

27.23

18.19

1,3-dicarbomethoxybenzene

25.30

38.79

1,3-dicarbomethoxynaphthalene

30.50

20.26

1,3-dichlorobenzene

12.64

12.59

1,3-dicyanopropane

14.33

1,3-difluorobenzene

9.40

1,3-dihydroxybenzene

20.10

1,3-diiodobenzene

1,3-dimethyluracil

1,3-dimethylurea

15.93

14.60

13.90

24.90

19.28

23.79

11.59

19.33

26.99

9.58

16.95

15.50

1,3-dinitro-1,3-diazacycloheptane

16.57

21.09

1,3-dinitro-1,3-diazacyclohexane

29.10

1,3-dinitro-1,3-diazacyclopentane

25.54

25.53

24.44

1,3-dinitro-5-nitroso-1,3,5-triazacyclohexane

25.97

30.37

1,3-dinitrobenzene

17.36

16.40

1,3-dioxolane

9.87

1,3-diphenylacetone

20.20

15.63

20.70

1,3-diphenylurea

34.60

31.74

1,3-dithiane

15.20

22.90

1,3-nitrochlorobenzene

1,4,5,8-tetracarbomethoxynaphthalene

1,4,5-tricarbomethoxynaphthalene

19.37

14.00

36.10

34.51

1,4,6-tricarbomethoxynaphthalene

26.50

29.15

30.20

29.15

1,4-[bis[(4-methylphenyl)amino]-9,10-anthracenedione

36.59

32.55

1,4-bis(diphenylphosphino)butane

45.30

49.43

1,4-bis(phenylglyoxaloyl)benzene

32.30

32.50

1,4-bis-[4-(4'-n-butylbiphenyl)]butane

37.74

49.26

1,4-bromochlorobenzene

1,4-bromoiodobenzene

18.76

12.58

$19.13 \quad 14.53$

1,4-cyclohexadiene

6.53

10.80

1,4-cyclohexanedione

17.15

17.87

1,4-diamino-2-methoxyanthraquinone

35.29

31.56

1,4-diaminoanthraquinone

24.20

27.71

1,4-dibromobenzene

20.04

13.57

1,4-dicarbomethoxybenzene

32.09

20.26

1,4-dicarbomethoxynaphthalene

20.40

23.79

1,4-dichloro-2,5-dimethoxybenzene

27.56

19.30

1,4-dichlorobenzene

18.16

11.59 
1,4-dihydroxybenzene

26.95

16.95

1,4-dihydroxybutane

$\begin{array}{ll}18.70 & 22.22 \\ 22.37 & 15.50\end{array}$

1,4-diiodobenzene

1,4-dimethylnaphthalene

$10.60 \quad 14.65$

1,4-dinitrobenzene

28.12

16.40

1,4-dioxane

15.20

17.96

1,4-dioxane-2,5-dione

$16.87 \quad 18.97$

1,4-di-tert-butylbenzene

22.48

16.06

1,4-dithiane

$21.60 \quad 22.90$

1,4-nitrochlorobenzene

11.85

14.00

1,4-pentadiene

1,5-cyclooctanedione

$6.14 \quad 3.35$

1,5-dicarbomethoxynaphthalene

22.53

1,5-dichloro-3-oxapentane

23.79

1,5-dimethyltetrazole

26.40

21.07

1,5-dinitro-3-nitroso-1,3,5-triazacycloheptane

8.39

13.65

1,5-pentanediol

1,6-bis-[4-(4'-ethylbiphenyl)]hexane

14.70

$\begin{array}{ll}30.89 & 32.70 \\ 15.72 & 25.17\end{array}$

$38.90 \quad 45.08$

1,6-dicarbomethoxynaphthalene

22.10

23.79

1,6-hexanediol

25.05

28.11

1,7-diacetoxy-2,4,6-trinitro-2,4,6-triazaheptane

38.49

50.67

1,7-dicarbomethoxynaphthalene

20.00

23.79

1,7-heptanediol

21.30

31.06

1,8-bis-(4-biphenyl)octane

56.00

46.44

1,8-bis-[4-(4'-ethylbiphenyl)]butane

46.00

37.46

1,8-bis-[4-(4'-ethylbiphenyl)]octane

50.40

49.26

1,8-bis-[4-(4'-n-butylbiphenyl)]octane

40.00

61.06

1,8-dimethylnaphthalene

15.77

14.65

1,8-octanediol

1,9-nonanediol

36.10

34.01

$10 \mathrm{H}$-phenothiazine

36.40

36.96

10-nonadecanone

26.92

24.89

10-octadecynoic acid

66.67

51.35

$52.32 \quad 47.21$

11-cyclohexyleicosane

48.70

64.75

11-heneicosanone

76.20

57.25

11-n-decylheneicosane

11-octadecynoic acid

71.13

84.44

11-phenyleicosane

55.97

47.21

12-octadecynoic acid

64.77

61.41

12-tricosanone

49.79

47.21

13-octadecynoic acid

78.03

63.15

$55.51 \quad 47.21$

14-octadecynoic acid

52.74

47.21

16-octadecynoic acid

60.10

49.55

17-octadecynoic acid

54.20

55.29

1-8-naphthalic anhydride

$23.32 \quad 20.16$

1a,2a,3ß,4a,5a,6ß-hexachlorocyclohexane

22.13

22.60

1 -acetoxynaphthalene

20.21

17.89

1-acetyl-2-naphthol

1-amino-4-hydroxy-2-phenoxy-9,10-anthracenedione

21.34

19.65

1 -aminoanthraquinone

30.79

34.01

28.78

25.49

1-aminopropane

11.80

1-benzoyl-2-naphthol

10.97

20.49

1-bromo-2-chloro-1,1,2-trifluoroethane

31.35

9.68 
1-bromo-2-chloroethane

1-bromobutane

9.23

15.28

1-bromodocosane

68.12

68.36

1-bromoheptane

21.76

24.13

1-bromohexane

18.05

21.18

1-bromonaphthalene

15.16

15.08

1-bromononane

30.12

30.02

1-bromooctane

24.69

27.07

1-bromopentane

14.37

18.23

1-bromotricosane

103.34

1-bromoundecane

33.47

91.95

1-butene

3.85

35.92

1-butyne

6.03

4.49

1-chloro-2-(2,2,2-trichloro-1-(4-chlorophenyl)ethyl)benzene

23.09

6.71

1-chloro-2-(2,2-dichloro-1-(4-chlorophenylethenyl)benzene

23.84

24.04

1-chloro-2,2-(bis-(4-chlorophenyl)ethylene

25.52

1-chlorodibenzodioxin

23.20

16.88

1-chloronaphthalene

12.90

15.35

17.81

1-cis-3-pentadiene

5.64

14.09

1-cyclohexyl-1-phenyldodecane

35.19

9.21

1-decanethiol

33.30

50.57

1-decanol

37.66

30.15

1-decene

22.10

33.51

1-deoxy-D-glucopyranose

27.40

1-docosanol

63.80

22.18

29.94

1H-1,2,4-triazol-3-amine

21.93

68.90

1-heptanethiol

25.40

21.04

1-heptanol

18.16

21.30

1-heptene

1-hexacosanol

12.64

24.67

84.52

13.34

1-hexadecanol

58.41

1-hexadecene

34.08

80.70

1-hexanethiol

18.03

51.21

1-hexanol

15.48

39.88

1-hexene

9.35

18.35

1-iodonaphthalene

1-methoxy-2-(2,2,2-tricloro-1-(4-methoxyphenyl)ethyl)benzene

15.91

22.45

18.03

1-methyl-7-isopropylphenanthrene

27.20

10.87

21.72

1-methylcyclohexanol

8.41

10.39

16.05

29.69

19.13

23.29

15.73

1-methylcyclopentanol

11.97

13.40

1-methylnaphthalene

15.70

32.82

13.86

1-methyltetrazole

1-naphthaleneacetamide

22.26

12.86

25.63

1-naphthaleneacetic acid

22.61

1-naphthoic acid

19.89

22.77

1-naphthyl benzoate

16.98

23.75

1-naphthyl methylcarbamate

24.51

24.86

1-naphthylamine

1-nitronaphthalene

15.53

18.43

16.58

16.50

1-nonanethiol

33.50

27.20

1-nonene

19.97

19.24

1-octanethiol

24.27

24.25 
1-octene

15.31

16.29

1-pentadecanol

54.73

48.26

1-pentanethiol

17.53

15.40

1-pentanol

10.50

18.77

1-pentene

5.81

7.44

1-propanethiol

9.95

9.51

1-propanol

5.37

12.87

1-tetradecanol

49.37

45.31

1 -undecene

26.48

25.13

2-((4-chloro-6-(cyclopropylamino)-1,3,5-triazin-2-yl)amino-

22.51

37.12

2-methylpropanenitrile

23.82

32.09

2-(1,3-dioxolan-2-yl)phenyl methylcarbamate

17.26

23.91

2-(1-methylethyl)phenyl methylcarbamate

26.14

23.07

2-(2,4,5-trichlorophenoxy)propanoic acid

39.58

27.06

2-(2,4-dichlorophenoxy)propanoic acid

30.43

26.03

2-(3,4-dichlorophenyl)-4-methyl-1,2,4-oxadiazolidine-3,5-dione

29.50

22.74

2-(3-hydroxy-2-quinolinyl)-1H-indene-1,3(2H)-dione

30.89

28.23

2-(4-chloro-2-methylphenoxy)propanoic acid

26.43

25.79

2-(6-methoxy-2-naphthyl)propionic acid

29.41

25.84

2-(dimethylamino)-1,2-diphenylethanone

22.38

22.03

2-(docosanoxy)ethanol

2-(hexadecyloxy)ethanol

56.85

76.37

2,11-dicyclohexyldodecane

52.26

58.67

2,2,2-trinitroethanol

43.93

49.31

19.73

2,2,2-trinitroethyl 4,4,4-trinitrobutyrate

22.59

39.61

2,2,2-trinitroethyl-4,4-dinitropentanoate

32.64

35.98

2,2',3,3',4,5,5',6,6'-nonachlorobiphenyl

26.78

23.99

2,2',3,3',5,5',6,6'-octachlorobiphenyl

22.60

22.96

2,2',3,3',5,5',6-heptachlorobiphenyl

22.80

21.93

2,2',3,3',5,5'-hexachlorobiphenyl

20.30

20.90

2,2',3,3',6,6'-hexachlorobiphenyl

29.20

20.90

2,2,3,3-tetramethylbutane

21.10

12.95

2,2,3,3-tetramethylpentane

12.44

15.90

2,2,3-trimethylbutane

13.41

9.53

2,2',4,4',6,6'-hexachlorobiphenyl

7.07

20.90

2,2,4,4-tetramethylpentan-3-ol

17.50

17.64

2,2,4,4-tetramethylpentane

9.63

15.90

2,2',4,5,5'-pentachlorobiphenyl

9.75

19.87

2,2',4',5-tetrachlorobiphenyl

18.80

18.84

2,2,4-trimethylpentane

23.40

12.48

2,2,5,5-tetramethylhex-3-ene

9.20

10.09

2,2,6,6-tetramethyl-1,3-dioxane

16.41

16.49

2,2-bis-(4-cyanatophenyl)propane

10.90

25.67

2,2-bis(phenylthio)propane

24.40

27.07

2,2-bis-hydroxymethylpropanoic acid

28.42

2,2-dichloropropane

45.89

11.40

2,2-dicyanopropane

9.91

12.69

2,2-dimethoxy-1,2-diphenylethanone

13.92

21.19

2,2-dimethyl-1,3-dioxan

20.86

17.22

2,2-dimethyl-1,3-propanediol

21.03

2,2-dimethyl-1-propanol

22.25

14.63

2,2-dimethylbutane

8.21

11.18 
2,2-dimethylheptane

2,2-dimethylpropanoic acid (pivalic acid)

11.36

15.62

2,2-dinitro-1,3-propanediol

21.34

23.02

2,2-dinitropropane

18.55

10.23

$22.44 \quad 16.63$

2,2-dinitropropyl-4,4-dinitropentanoate

32.09

32.88

2,2-dintropropyl-4,4,4-trinitrobutyrate

63.26

32.06

2,2'-methylenebis(3,4,6-trichlorophenol)

33.26

29.47

2,3,4,5,6-pentachlorobiphenyl

21.35

19.87

2,3,4,5,6-pentafluorotoluene

13.28

10.44

25.20

18.84

2,3,4-trimethylpentane

9.27

7.40

2,3,5,6-tetrachloro-2,5-cyclohexadiene-1,4-dione

30.87

23.32

2,3,5-tricarbomethoxynaphthalene

41.00

29.15

2,3,5-triiodobenzoic acid

32.23

26.89

2,3,6,7,10,11-hexakis(1-decynyl)triphenylene

63.00

129.05

2,3,6,7-tetracarbomethoxynaphthalene

42.20

34.51

2,3,6-tricarbomethoxynaphthalene

34.40

29.15

2,3,6-trichlorobenzoic acid

23.85

19.73

2,3,6-trichlorophenylacetic acid

22.43

22.17

2,3-benzofluorene

2,3-dicarbomethoxynaphthalene

23.40

19.04

20.20

23.79

2,3-dichloro-1,4-naphthalenedione

28.53

21.80

2,3-dichlorophenol

21.36

15.11

2,3-dihydro-2,2-dimethyl-7-benzofuranol-3-one

21.79

20.61

2,3-dihydro-2,2-dimethylbenzofuran-7-yl methylcarbamate

30.33

26.74

2,3-dihydro-6-methyl-5-phenylcarbamoyl-1,4-oxathiin-4,4-dioxide

26.66

2,3-dimethyl-2,3-bis(4-tert-butylphenyl)butane

43.93

21.09

27.48

28.60

2,3-dimethyl-2,3-bis(phenylazo)butane

14.70

25.33

2,3-dimethyl-2,3-butanediol

25.52

17.92

22.07

2,3-dimethyl-2-butene

9.97

9.76

2,3-dimethylbenzoic acid

18.30

20.82

2,3-dimethylbutane

7.69

6.11

2,3-dimethylnaphthalene

15.90

14.65

21.02

14.83

2,3-dimethylpyridine

13.48

11.92

2,3-dinitrophenol

26.24

18.82

2,3-dinitrotoluene

17.57

17.20

2,3-pentadiene

2,4,4-trimethyl-1-pentene

6.61

5.60

$8.79 \quad 11.51$

2,4,4-trimethyl-2-pentene

6.78

9.93

$30.00 \quad 21.92$

$25.10 \quad 17.10$

2,4,5-tribromostyrene

17.10
17.81

2,4,5-trichlorobiphenyl

22.80

17.81
16.14

2,4,5-trichlorophenol

21.59

16.14
12.73

2,4,5-trimethylthiazole

9.00

24.70

20.63

2,4,5-trinitrotoluene

19.33

24.61

2,4,6-N-tetranitroethylaniline

30.21

2,4,6-tribromophenol

23.51

18.91 
2,4,6-trichlorobiphenyl

2,4,6-trimethylpyridine

2,4,6-trinitro-1,3-dimethylbenzene

2,4,6-trinitroresorcinol

2,4,6-trinitrotoluene

2,4-dimethylpentane

2,4-dinitrotoluene

2,5,8,11-tetraoxadodecane

2,5-dichlorophenol

2,5-diethoxy-1,4-benzoquinone

2,5-dimethylaniline

2,5-dimethylphenol

13.70

14.63

23.38

14.83

2,5-dimethylpyridine

2,5-dimethylpyrrole

2,5-dimethyltetrazole

13.50

13.65

2,5-dimethylthiophene

2,5-di-n-heptadecyloxy-1,4-benzoquinone

11.13

2,5-di-n-heptyloxy-1,4-benzoquinone

133.90

59.30

119.03

2,5-di-n-hexadecyloxy-1,4-benzoquinone

60.05

2,5-di-n-hexyloxy-1,4-benzoquinone

122.90

113.13

45.45

53.41

2,5-dinitrophenol

2,5-di-n-nonadecyloxy-1,4-benzoquinone

18.82

23.73

130.83

79.30

71.85

52.40

65.95

2,5-di-n-octyloxy-1,4-benzoquinone

123.40

107.23

2,5-di-n-undecyloxy-1,4-benzoquinone

93.40

83.64

2,5-dipentoxy-1,4-benzoquinone

2,5-dipropoxy-1,4-benzoquinone

47.68

47.51

44.70

35.72

2,6-dichloro-4-benzenamine

29.48

15.11

32.64

18.16

26.17

15.73

$12.60 \quad 16.78$

2,6-dichlorobiphenyl

22.14

14.92

2,6-dichlorophenol

2,6-diisopropylphenol

2,6-dimethylnaphthalene 
2,6-dinitro-N,N-dipropyl-4-(trifluoromethyl)benzenamine

2,6-dinitrotoluene

2,6-di-tert-butyl-4-methoxyphenol

2,6-di-tert-butyl-4-methylphenol

2,6-di-tert-butylphenol

2,7-dicarbomethoxynaphthalene

2-[[4-chloro-6-(ethylamino)-1,3,5-triazin-2-yl]amino]-

2-methylpropanenitrile

41.96

37.26

2-[bis(2-chloroethyl)amino]tetrahydro- $2 \mathrm{H}-1$,

3,2-oxazophosphorine-2-oxide

33.13

31.61

2-acetoxynaphthalene

20.05

17.89

2-acetyl-1-naphthol

22.52

20.95

2-acetylamino-9-[(2-hydroxyethoxy)methyl]-1,9-dihydro-6H-purin-6-one

53.83

47.04

2-acetylamino-9-[(2-hydroxyethoxy)methyl]-9H-purine

54.92

41.39

2-amino-2-hydroxymethylpropane-1,3-diol

29.21

2-amino-2-methylpropane-1,3-diol

35.64

22.81

2-amino-9-[(2-acetoxyethoxy)methyl]-1,9-dihydro-6H-purin-6-one

30.49

40.69

2-amino-9-[(2-hydroxyethoxy)methyl]-1,9-dihydro-6H-purin-6-one

49.90

38.62

2-amino-9-[(2-hydroxyethoxy)methyl]-9H-purine

30.44

32.98

2-aminobenzoic acid

42.20

20.16

2-aminobiphenyl

20.50

18.24

$\begin{array}{rr}7.33 & 7.20\end{array}$

2-benzoyl-1-naphthol

20.18

21.79

2-bromo-2-chloro-1,1,1-trifluoroethane

4.84

9.68

2-bromobutane

2-bromonaphthalene

2-bromopropane

6.88

10.67

20.35

6.55

7.92

6.48

2-butanethiol

2-butanol

5.97

8.44

15.08

7.72

11.56

7.85

11.16

2-butanone

9.25

9.45

2-butyne

27.10

3.30

2-carbomethoxynaphthalene

2-chloro-1-(3-ethoxy-4-nitrophenoxy)-4-(trifluoromethyl)benzene

30.07

18.43

26.54

$13.01 \quad 12.67$

2-chloro-6-(trichloromethyl)pyridine

20.30

16.26

2-chloro-9-(3-dimethylaminopropylidene)-10-thioxanthene

27.82

23.11

2-chloroanthraquinone

39.00

24.30

2-chlorobenzoic acid

25.73

14.54

18.97

2-chlorobiphenyl

23.10

15.76

17.81

$14.79 \quad 19.96$

2-chloroethylphosphonic acid

25.31

25.64

2-chloronaphthalene

2-chloro-N-isopropyl N-phenylacetamide

14.70

14.09

26.05

19.59

2-chloro-N-isopropylacetamide

20.14

2-chlorophenol

26.05

14.08

2-chloropropane

12.61

7.39

6.90

2-chlorothiophene

8.97

10.58 
2-cyano-2-methylpropane

11.43

11.81

2-cyclohexyl-4,6-dinitrophenol

27.93

2-cyclohexylcyclohexanone

28.03

25.08

2-deoxy-2-fluoro-D-glucopyranose

18.00

29.56

2-deoxy-D-glucopyranose

38.20

29.94

2-ethoxyisonitrosoacetanilide

35.59

2-ethyl-2-diphenylmethyl-1,3-cyclopentanedione

29.87

2-fluorenyl-2-methyl-1,3-cyclohexanedione

23.00

28.20

24.91

2-fluorenyl-2-methyl-1,3-cyclopentanedione

35.70

22.58

2-fluorotoluene

24.60

10.35

2-heneicosanone

9.80

59.58

2-heptanone

77.65

18.30

2-hexadecanoyloxy-1,3-bis-(9-cis-octadecenoyloxy)propane

19.71

138.04

2-hexanone

14.90

15.35

2-hydroxybenzoic acid

24.60

20.35

2-hydroxymethyl-2-methyl-1,3-propanediol

36.15

27.43

2-iodobenzoic acid

21.38

20.92

2-iodonaphthalene

16.04

16.05

2-isopropoxyphenyl N-methylcarbamate

22.96

16.92

26.47

2-methoxy-4H-1,3,2-benzodioxaphosphorin 2-sulfide

17.30

2-methyl-1,3-butadiene

4.92

6.91

7.91

6.80

2-methyl-1-phenyl-2-(N-piperidinyl)-1-propanone

16.74

26.41

2-methyl-1-propanol

6.32

27.12

11.21

2-methyl-2-(methylsulfonyl)propanal oxime

21.45

22.71

29.44

2-methyl-2-butanethiol

7.67

10.80

2-methyl-2-butanol

8.21

13.67

2-methyl-2-butene

7.59

7.45

34.30

26.92

2-methyl-2-diphenylmethyl-1,3-cyclopentanedione

34.83

23.37

2-methyl-2-nitro-1-propanol

23.76

16.97

29.96

32.41

2-methyl-4,6-dinitrophenol

19.41

19.61

2-methylbutane

5.13

6.91

8.87

15.46

2-methylcyclothiapentane

2-methyldecane

2-methylfuran

25.06

8.55

11.92

25.46

2-methylheptane

9.18

9.69

16.61

2-methylhexane

18.11

13.66

2-methylnaphthalene

17.49

13.86

2-methylnonane

6.27

18.58

2-methylpiperidine

9.72

22.51

10.71

14.37

2-methylpyridine

12.37

11.13

2-methyltetrazole

12.16

12.86

2-methylthiazole

9.47

11.14

2-methylthiophene

23.54

10.34

2-naphthoic acid

26.23

22.77

2-naphthyl benzoate

23.33

23.75

16.58

2-n-butyl-5-(4-bromobiphenyl-4-yl)thiophene

21.40

29.26

2-nitro-1-(4-nitrophenoxy)-4-(trifluoromethyl)benzene

18.44

22.14 
2-nitro-5-methylphenol

20.79

16.18

2-nitroaniline

16.11

15.19

2-nonadecanone

68.65

53.69

2-n-propyl-5-(4-bromophenyl)thiophene

15.70

26.31

2-octanone

24.42

21.25

2-pentadecanone

54.39

41.89

2-pentanol

8.48

14.11

2-pentanone

11.06

12.40

2-phenylbenzimidazole

22.18

24.16

2-piperadone

2-propanethiol

16.10

23.37

2-propanol

5.78

4.90

2-pyrrolidone

5.41

8.21

$13.92 \quad 21.04$

2-sec-butyl-4,6-dinitrophenol

21.81

22.22

2-sec-butyl-4,6-dinitrophenyl 3-methylcrotonate

18.89

32.25

2-tetradecanone

49.12

38.94

2-undecanone

28.78

30.09

3-(1-methylethyl)-(1H)-2,1,3-benzothiadiazin-4(3H)-one 2,2-dioxide

21.77

22.15

3-(3,4-dichlorophenyl)-1,1-dimethylurea

33.89

27.08

3-(4-chlorophenyl)-1,1-dimethylurea

29.46

26.06

3-(5-(1,1-dimethylethyl)-1,3,4-thiadiazol-2-yl)-4-hydroxy-1-methyl-

25.46

24.22

2-imidazolidinone

3(n-decylamino)-1,2-propanediol

54.80

48.81

3(n-decyloxy)-1,2-propanediol

38.90

45.67

3(n-decylthio)-1,2-propanediol

34.60

46.91

3(n-dodecylamino)-1,2-propanediol

62.10

54.71

3(n-dodecyloxy)-1,2-propanediol

51.40

51.57

3(n-dodecylthio)-1,2-propanediol

38.40

52.81

3(n-heptylamino)-1,2-propanediol

28.80

39.96

3(n-heptyloxy)-1,2-propanediol

3(n-heptylthio)-1,2-propanediol

25.62

36.82

29.28

38.06

3(n-hexyloxy)-1,2-propanediol

10.20

33.87

3(n-hexylthio)-1,2-propanediol

48.50

35.11

3(n-nonylamino)-1,2-propanediol

53.20

45.86

3-(n-nonyloxy)-1,2-propanediol

29.50

42.72

3(n-octylamino)-1,2-propanediol

3(n-octyloxy)-1,2-propanediol

45.10

42.91

33.40

39.77

3(n-octylthio)-1,2-propanediol

39.80

41.01

3(n-tetradecylamino)-1,2-propanediol

64.90

60.61

3(n-tetradecyloxy)-1,2-propanediol

62.10

57.46

3(n-tetradecylthio)-1,2-propanediol

43.10

58.71

3(n-tridecylamino)-1,2-propanediol

3(n-tridecyloxy)-1,2-propanediol

3(n-tridecylthio)-1,2-propanediol

68.70

57.66

51.40

54.51

34.00

55.76

3(n-undecylamino)-1,2-propanediol

58.20

51.76

3(n-undecyloxy)-1,2-propanediol

43.10

48.62

$30.30 \quad 49.86$

3-(p-tolyl-4-sulfonyl)-1-butyl urea

25.61

33.20

3,3',4'4'-tetraaminodiphenyl ether

26.71

29.13

42.97

36.73

3,3'-bis-(1-cyclohexylethyl)-5,5'-dimethyldiphenylmethane-2,2'-diol

29.29

53.59

16.95

20.65

3,3-diethylpentane

11.57

20.03 
3,3-dimethyl-1-(methylthio)-2-butanone O-methylcarbamoyloxime

19.83

26.73

3,3-dimethyl-1-butene

3,3-dimethyl-2-butanone

11.31

3,3-dimethylpentane

11.34

14.13

3,3-dimethylpentanedioic anhydride

7.07

16.17

3,3'-di-tert-butyl-5,5'-dimethyl-2,2-dihydroxydiphenylmethane

17.99

31.79

3,4-benzophenanthrene

29.33

20.12

3,4-benzopyrene

16.32

20.48

3,4-dichloro-2-methoxybenzoic acid

27.21

23.66

3,4-dichlorophenol

35.33

15.30

3',4'-dichloropropionanilide

20.93

24.14

3,4-diethyl-3,4-bis(4-tert-butylphenyl)hexane

18.26

40.40

3,4-dimethylisoxazol 5-sulphanylamide

29.71

$\begin{array}{ll}28.01 & 26.85 \\ 18.13 & 14.83\end{array}$

3,4-dimethylphenol

3,4-dimethylphenyl methylcarbamate

24.97

22.91

3,4-dimethylpyridine

14.70

11.92

3,4-dinitrophenol

25.37

20.11

3,4-dinitrotoluene

18.83

3,5,6-trichloro-2-pyridinol

25.79

17.20

16.94

3,5,6-trichloro-2-pyridinyloxyacetic acid

31.17

27.53

3,5-dibromo-4-hydroxybenzonitrile

21.03

3,5-dichlorobenzoic acid

32.03

21.29

3,5-dichloro-N-(1,1-dimethyl-2-propynyl)benzamide

22.97

21.29
26.53

3,5-dichlorophenol

3,5-diisopropylphenol

28.68

15.30

12.13

16.74

3,5-dimethyl-4-(dimethylamino)phenyl methylcarbamate

25.65

3,5-dimethylbenzoic acid

20.82

3,5-dimethylphenol

18.37

22.60

14.83

3,5-dimethylpyridine

18.00

11.92

3,5-dinitrobenzoic acid

3,6-dichloro-2-methoxybenzoic acid

13.11

22.80

26.11

3,6-dichloro-5-hydroxy-2-methoxybenzoic acid

23.66

3-amino-2,5-dichlorobenzoic acid

22.90

23.32

3-aminoacetophenone

37.42

17.22

3-aminobenzoic acid

29.00

3-bromopentane

3-chloro-1,1,1,3,3-pentafluoropropane

21.84

22.75

$8.40 \quad 13.62$

$10.47 \quad 10.06$

$23.85 \quad 20.26$

3 -chlorobenzoic acid

14.27

3-chlorophenol

14.91

29.56

-Dlucopyranose

18.30

29.94

3-diphenylmethyl-2,4-pentanedione

32.60

24.76

3-ethyl-3-diphenylmethyl-2,4-pentanedione

27.02

26.75

3 -ethylheptane

34.70

19.56

3-ethylpentane

16.02

13.66

3-fluorotoluene

10.35

3-heptanone

8.30

15.97

3-hexanone

17.53

13.02

3-hydroxybenzoic acid

14.50

22.94

3-iodobenzoic acid

26.20

22.22

3-methyl-1,2-butadiene

6.64

3-methyl-1-butanethiol

28.70
7.95

10.80

3-methyl-1-butene

7.41

4.83 
3-methyl-1-phenyl-1H-pyrazol-5-yl dimethylcarbamate

3-methyl-2-butanethiol

3-methyl-3-diphenylmethyl-2,4-pentanedione

3-methylcyclothiapentane

10.37

15.46

3-methylheptane

3-methylpentane

3-methylpyridine

3-nitroaniline

3-nitrophthalic anhydride

23.68

16.48

3-nitrotoluene

18.40

20.06

3-pentanol

14.98

13.76

3-pentanone

9.08

14.11

3-propyl-3-diphenylmethyl-2,4-pentanedione

11.71

10.07

$27.10 \quad 29.70$

4-(1,1-dimethylethyl)-n-(1-methylpropyl)-2,6-dinitrobenzeneamine

20.84

26.77

4-(2,4,5-trichlorophenoxy)butanoic acid

30.28

32.62

4-(2,4-dichlorophenoxy)butyric acid

38.42

31.59

4-(2-chlorophenylhydrazone)-3-methyl-5-isoxazolone

4-(4-chloro-2-methylphenoxy)butanoic acid

28.04

26.90

$32.02 \quad 31.35$

$31.88 \quad 22.75$

4-(6-hexenyloxy)-3',4'difluorodiphenyldiacetylene

37.45

30.47

4-(cis-3-hexenyloxy)-3',4'difluorodiphenyldiacetylene

30.97

26.46

4-(cis-4-hexenyloxy)-3',4'difluorodiphenyldiacetylene

4-(dipropylamino)-N,N-dimethyl-3,5-dinitrobenzenesulfonamide

35.32

28.79

32.57

38.06

4-(N,N-dipropylamino)-3,5-dinitrobenzenesulphonamide

38.48

33.15

4,4'-di-(2-methoxyethoxy)biphenyl

40.20

37.37

24.40

20.44

4,4'-didecanoyloxydiphenyldiacetylene

87.10

68.93

4,4'-didodecanoyloxydiphenyldiacetylene

94.20

80.72

4,4'dihydroxydiphenyl-2,2-propane

30.10

22.09

4,4'-dinitrodiphenyl ether

10.29

22.70

4,4'-diundecanoyloxydiphenyldiacetylene

61.89

74.82

$31.48 \quad 22.96$

29.18

42.86

4'4'-diaminodiphenyl ether

7.74

22.86

4-acetoxybenzoic acid

26.35

24.06

4-amino-6-(1,1-dimethylethyl)-3-(methylthio)1,2,4-triazin-5(4H)-one

18.00

21.45

$38.00 \quad 17.22$

$20.92 \quad 22.75$

4-aminobenzoic acid

22.30

30.24

4-amino-N-(6-methoxy-3-pyridazinyl)benzenesulfonamide

20.07

13.85

4-benzoyl-1-naphthol

28.64

21.79

4-bromo-2,5-dichloroaniline

22.11

16.93

22.11

17.13

4-chloroazobenzene

27.20

16.83

$32.26 \quad 20.26$

4-chlorobenzoic acid

13.32

15.76

4-chlorobut-2-ynyl 3-chlorophenylcarbamate

26.91

25.45

4-chlorophenol

4-chlorophenoxyacetic acid

14.07

14.27

$36.27 \quad 24.66$

4-chlorophenyl 4-chlorobenzenesulfonate

20.37

4-chlorophenylbenzenesulfonate

23.63

19.35

4-ethoxy-4'fluorodiphenyldiacetylene

21.44

23.58 
4-ethoxy-4'-trifluoromethyldiphenyldiacetylene

4-ethoxybenzoic acid

4-ethoxyisonitrosoacetanilide

$7.60 \quad 35.59$

4-ethoxyphenylacetic acid

23.00

25.89

4-ethylbenzoic acid

14.06

20.64

4-ethynyl-1-[(4-ethynylphenyl)methoxy]benzene

21.20

26.09

4-fluorotoluene

9.35

10.35

4-heptanone

$16.16 \quad 15.97$

4-hexylresorcinol

$19.04 \quad 30.16$

4-hydroxy-3,5-diiodobenzonitrile

33.63

30.16
22.96

4-hydroxyazobenzene

4-hydroxybenzoic acid

32.99

19.51

4-hydroxyphenylacetic acid

30.90

22.94

4-hydroxyphenylpropionic acid

28.40

22.79

4-iodobenzoic acid

28.90

24.90

4-methoxybenzoic acid

35.24

22.22

4-methoxyphenol

4-methoxyphenylacetic acid

28.40

23.09

18.30

17.10

4-methoxyphenylbutyric acid

21.80

18.22

25.30

27.99

4-methoxyphenylpropionic acid

28.50

25.04

4-methyl-7-aminocoumarin

32.09

22.15

4-methyl-7-diethylaminocoumarin

17.88

25.54

4-methyl-7-dimethylaminocoumarin

23.92

24.34

4-methyl-7-hydroxycoumarin

29.14

22.34

4-methylcyclohex-1-ene

6.63

11.74

4-methylheptane

10.84

16.61

4-methylpent-1-ene

4.93

4-methylphenanthrene

14.09

5.78

4-methylsulphonyl-2,6-dinitro-N,N-dipropylaniline

28.05

17.39

4-methylthiazole

4-methylthio-3,5-xylyl methylcarbamate

4-n-butoxy-2',3',4'-trifluorodiphenylacetylene

8.90

30.36

36.00

25.40

4-n-butoxy-4'-trifluoromethyldiphenyldiacetylene

25.37

22.26

25.30

24.33

18.50

32.20

27.00
22.80

35.95

11.14

26.32

28.23

28.19

28.89

4-n-butyl-3',4'-difluorodiphenylacetylene

4-n-butyl-3',4'-difluorodiphenyldiacetylene

4-n-butyl-4'-fluorodiphenylacetylene

31.94

22.81

24.10

22.79

22.33

22.31

4-n-ethoxy-4'-fluorodiphenylacetylene

4-n-ethyl-3',4'-difluorodiphenylacetylene

4-n-hexyl-3',4'-difluorodiphenylacetylene

4-n-hexylbenzoic acid

16.60

22.29

24.30

16.91

20.22

30.00

32.44

4-n-hexyloxy-2',3',4'-trifluorodiphenylacetylene

30.80

34.13

4-n-hexyloxy-2',4'-difluorodiphenylacetylene

34.10

34.11

4-n-hexyloxy-3',4'-difluorodiphenylacetylene

33.10

34.11

4-n-hexyloxy-4'-trifluoromethyldiphenyldiacetylene

33.98

34.79

4-nitro-4'-methylbenzylidene aniline

27.30

16.27

4-nitro-5-methylphenol

27.40

17.47

4-nitroaniline

21.09

16.48

4-nitrophthalic anhydride

17.14

20.06

4-nitrotoluene

16.81

13.76 
4-n-octyloxy-N-(3,5-dimethoxybenzylidene)aniline

44.25

4-n-octyloxy-N-(3,5-dimethylbenzylidene)aniline

35.30

38.12

4-n-octyloxy-N-(4-methoxybenzylidene)aniline

37.73

40.39

4-n-pentoxy-2',3',4'-trifluorodiphenylacetylene

42.29

31.18

4-n-pentoxy-4'-fluorodiphenylacetylene

33.10

31.13

4-n-pentoxybenzoic acid

27.20

34.89

4-n-pentyl-3',4'-difluorodiphenylacetylene

25.21

25.76

4-n-pentyl-3',4'-difluorodiphenyldiacetylene

22.10

27.05

4-n-pentyl-4'-fluorodiphenylacetylene

30.86

25.74

4-n-pentylbenzoic acid

4-n-propoxy-2',3',4'-trifluorodiphenylacetylene

25.60

29.49

4-n-propoxy-2',4'-difluorodiphenylacetylene

16.38

25.28

4-n-propoxy-4'-fluorodiphenylacetylene

26.10

25.26

4-n-propoxybenzoic acid

25.20

25.24

4-n-propyl-3',4'-difluorodiphenylacetylene

28.12

28.99

20.20

19.86

4-n-propyl-3',4'difluorodiphenyldiacetylene

22.03

21.15

4-n-propyl-4'-fluorodiphenylacetylene

24.10

19.84

4-octadecynoic acid

4-oxaheptane

57.94

47.21

4-propoxy-4'-trifluoromethyldiphenyldiacetylene

$\begin{array}{ll}10.77 & 16.89 \\ 18.81 & 25.94\end{array}$

4-propylbenzoic acid

$28.07 \quad 23.59$

4'-propylbiphenyl-4-carbonitrile

22.70

23.22

4-tert-butylbenzoic acid

17.91

22.50

4-tert-butylphenol

4-trans-(3-fluoro-4-cyanophenyl)cyclohexyl (E)-but-2-enoate

14.52

16.51

$21.10 \quad 31.68$

$28.40 \quad 29.54$

$30.20 \quad 28.55$

4-trans-(4-chlorophenyl)cyclohexyl (E)-2-butenoate

$25.10 \quad 27.55$

4-trans-(4-fluorophenyl)cyclohexyl (E)-2-butenoate

4-trans-(4-fluorophenylethyl)cyclohexyl (E)-butenoate

25.00

32.59

4-trans-(trifluoromethoxyphenyl)cyclohexyl (E)-but-2-enoate

21.60

32.27

4-trans-cyanocyclohexyl (E) 2-butenoate

24.40

26.53

5 '-(trifluoromethanesulphonamide)acet-2',4-xylidide

37.66

31.54

5,5-bis(3,3-dimethylbutyl)2,2,8,8-tetramethylnonane

5,5-dimethylperhydro-1,3-oxazine-2-one

48.53

50.68

$28.50 \quad 30.13$

$5,6,7,8$-tetrahydroquinoline

5,6-dibutyl-5,6-bis(4-tert-butylphenyl)decane

5-[2-chloro-4-(trifluoromethyl)phenoxy]-2-nitrobenzoic acid

9.08

16.83

$\begin{array}{ll}43.10 & 63.99\end{array}$

$37.67 \quad 28.79$

$26.75 \quad 29.27$

5 -amino-4-chloro-2-phenyl-3(2H)-pyridazinone

22.02

25.11

5-butyl-2-ethylamino-6-methylpyrimidin-4-ol

20.32

33.28

5-chloro-3-(1,1-dimethylethyl)-6-methyl-2,4(1H,3H)-pyrimidinedione

12.51

23.45

5 -isopropyl-m-tolyl methylcarbamate

23.04

23.86

5-methyl N-(methylcarbamoyloxy)thioacetimidate

21.73

26.69

24.07

17.17

5-methylnonane

16.65

22.51

5-methyltetrazole

16.00

19.21

5-methylthiazole

7.65

11.14

5-nonanone

5-octadecynoic acid

26.22

21.86

6-(4-biphenyl)-1-hexene

54.41

47.21

$15.10 \quad 23.00$

6,8,9-trimethyladenine

23.10

24.17

6,9-dimethyl-8-butyladenine

36.00

30.68

6-deoxy-6-fluoro-D-glucopyranose

27.20

29.50 
6-deoxy-D-glucopyranose

22.70

26.28

6-methyl-1,3-dithiolo[4,5-b]quinoxalin-2-one

29.92

22.16

6-octadecynoic acid

54.92

47.21

7,8-benzoquinoline

14.10

17.40

7-octadecynoic acid

53.61

47.21

8-(4-biphenyl)-1-octene

21.00

28.90

8-[4-(4'-n-butylbiphenyl)]-1-octene

12.40

36.21

8-octadecynoic acid

$\begin{array}{ll}55.30 & 47.21\end{array}$

9-fluorenone

18.12

18.34

9-heptadecanone

9H-pyrido[3,4-b]indole

9-methylfluorene

66.68

45.46

25.50

22.50

9-octadecynoic acid

16.32

11.29

54.87

47.21

a-(trifluoromethoxy)-a,a-difluoromethyl acetate

8.51

a,a'-dibromo-m-xylene

23.69

15.73

a,a'-dibromo-o-xylene

26.78

18.17

a, a'-dichloro-m-xylene

19.51

18.17

a, a'-dichloro-o-xylene

21.26

a,a'-dichloro-p-xylene

23.97

a-alanyl-a-alanine (DL)

33.20

21.46

acenaphthene

acenaphthylene

15.35

5.46

15.60

acetamide

21.67

acetanilide

5.72

9.06

acetonitile

acetylacetone enol

14.50

16.53

16.53

16.53

33.08

14.90

13.31

8.79

16.17

21.46

8.83

9.33

acetylene

7.18

12.29

a-chloroacetic acid

14.11

8.89

a-chloroacetic acid

16.30

15.86

acridine

acrylamide

18.58

15.86

17.40

15.33

16.86

acrylic acid

11.16

16.12

acrylonitrile

7.42

10.55

a-D-glucopyranose (a-D-glucose)

34.30

32.68

a-D-glucose

31.42

32.68

34.85

29.91

alanylglycine (with decomp)

56.60

32.74

allobarbitiol

32.31

26.63

a-methylacrylic acid

8.06

15.09

a-methylstyrene

a-naphthol

11.92

10.03

a-naphthyl acetate

23.01

16.77

20.21

22.61

androstanolone

27.06

22.09

aniline

10.54

13.05

anisaldazine

29.75

28.63

anthracene

29.37

16.59

anthraquinone

32.57

23.27

azelaic acid

32.67

38.76

azoxybenzene

18.89

$17.53 \quad 13.06$

b(4-chlorophenoxy)-a-(1,1-dimethylethyl)-1H-1,2,4-triazole-1-ethanol

24.47

32.88 


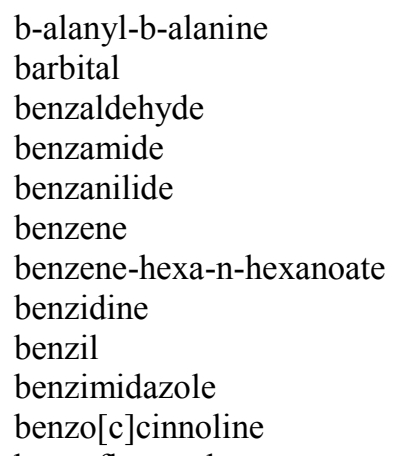


chlorocyclopentane

chlorodifluoromethane

4.19

13.09

chloroethane

4.45

2.81

chloroethyl methacrylate

17.00

8.56

chloropentafluorobenzene

14.29

18.74

chlorotrifluoroethylene

5.55

10.67

cholesterol*

30.91

5.66

chroman

16.26

29.96

chromone

17.31

18.02

chrysene

29.37

18.49

cinnamic acid

22.63

cinnamic anhydride

32.77

15.73

26.78

cis,cis-1,3,5-tri-tert-butylcyclohexane

17.99

cis,trans-1,3,5-tri-tert-butylcyclohexane

23.83

cis-1,2-cyclohexanediol

12.33

cis-1,2-dimethylcyclohexane

cis-1,2-dimethylcyclopentane

cis-1,3-dimethylcyclohexane

11.97

10.82

9.29

cis-1,4-di-tert-butylcyclohexane

8.79

7.31

cis-2-butene

cis-2-hexene

cis-2-pentene

8.88

7.11

cis-3-chloro-2-butenoic acid

13.81

cis-6-octadecenoic acid

47.50

cis-9-octadecenoic acid

39.60

12.57
19.20

20.12

24.57

25.91

16.39

26.16

26.16

19.46

12.67

10.34

12.67

12.67

21.63

cis-crotonic acid

coronene

cortisone

cortisone acetate

36.86

38.43

19.14

8.76

5.14

8.71

5.76

18.64

49.06

49.06

coumarin

22.90

17.39

21.18

29.89

cyanamide

cyanoacetamide

13.48

31.96

17.92

cyanocyclohexane

9.80

cyanogen

cyclobutane

8.11

21.11

17.66

8.23
12.62

10.86

cycloheptane

12.62
8.00

9.32

cycloheptanol

cycloheptatriene

3.51

16.31

cycloheptene

9.14

19.05

cyclohexane

cyclohexanethiol

12.81

11.54

14.72

10.00
11.67

13.98

cyclohexanol

10.95

13.41

cyclohexanone

10.95
9.69

16.72

cyclohexanone oxime

15.93

cyclohexene

8.46

17.71

cyclohexylbenzene

15.30

12.39

cycloocta-1,5-diene

9.83

14.08

18.65

cyclooctane

4.45

15.46

cyclooctanol

11.25

18.64

cyclooctatetraene

15.16

cyclooctene

12.28

17.05 


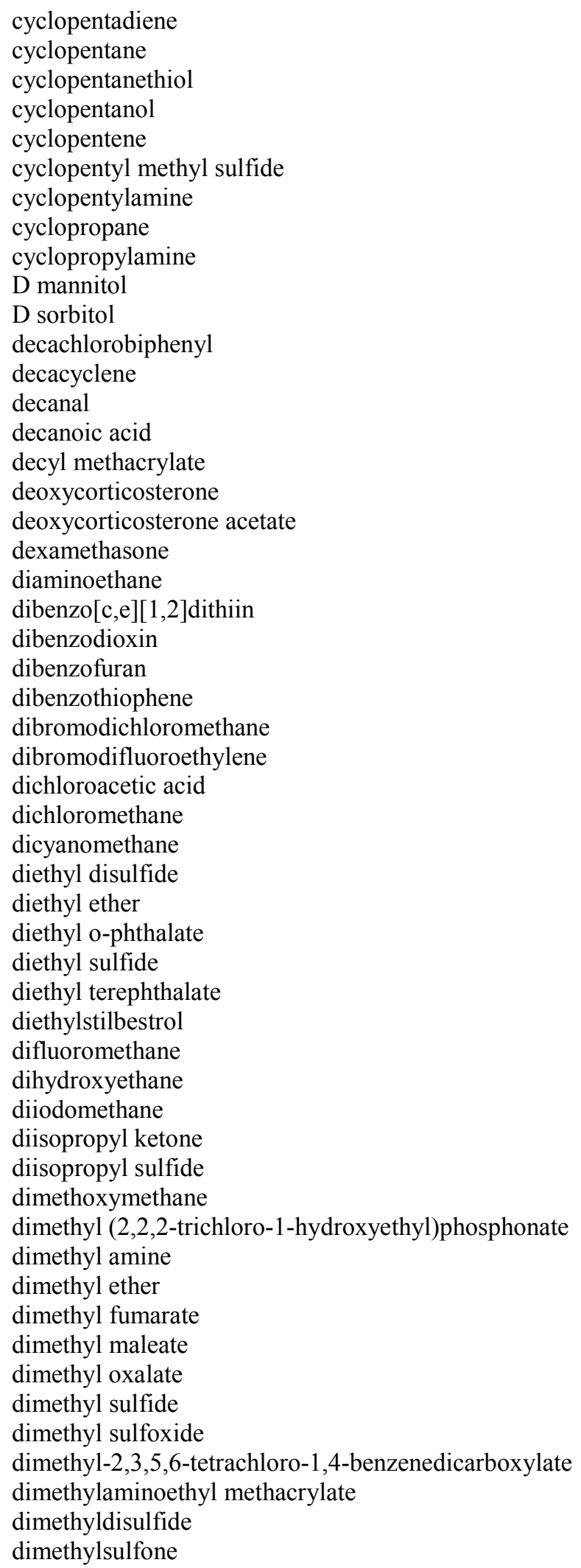

$\begin{array}{rr}8.01 & 8.47 \\ 8.26 & 11.65 \\ 7.83 & 11.08 \\ 6.25 & 14.39 \\ 4.12 & 10.06 \\ 10.10 & 13.81 \\ 8.79 & 16.40 \\ 5.44 & 6.99 \\ 13.18 & 11.74 \\ 56.10 & 35.07 \\ 30.20 & 35.07 \\ 39.34 & 25.02 \\ 25.40 & 31.77 \\ 30.60 & 30.05 \\ 27.82 & 34.41 \\ 30.55 & 33.77 \\ 27.98 & 25.54 \\ 29.66 & 26.07 \\ 42.02 & 27.97 \\ 23.07 & 14.19 \\ 19.30 & 18.22 \\ 23.20 & 16.78 \\ 18.60 & 15.10 \\ 21.58 & 16.61 \\ 8.48 & 10.74 \\ 7.04 & 8.64 \\ 12.34 & 17.27 \\ 6.16 & 9.63 \\ 10.80 & 14.27 \\ 9.40 & 15.04 \\ 7.19 & 10.99 \\ 17.99 & 26.16 \\ 11.92 & 12.23 \\ 24.69 & 26.16 \\ 31.63 & 27.58 \\ 4.36 & 6.00 \\ 9.96 & 16.32 \\ 12.05 & 15.10 \\ 11.20 & 10.74 \\ 10.42 & 8.92 \\ 8.33 & 9.61 \\ 25.00 & 24.87 \\ 5.37 & 20.99 \\ 4.94 & \\ 35.15 & \end{array}$




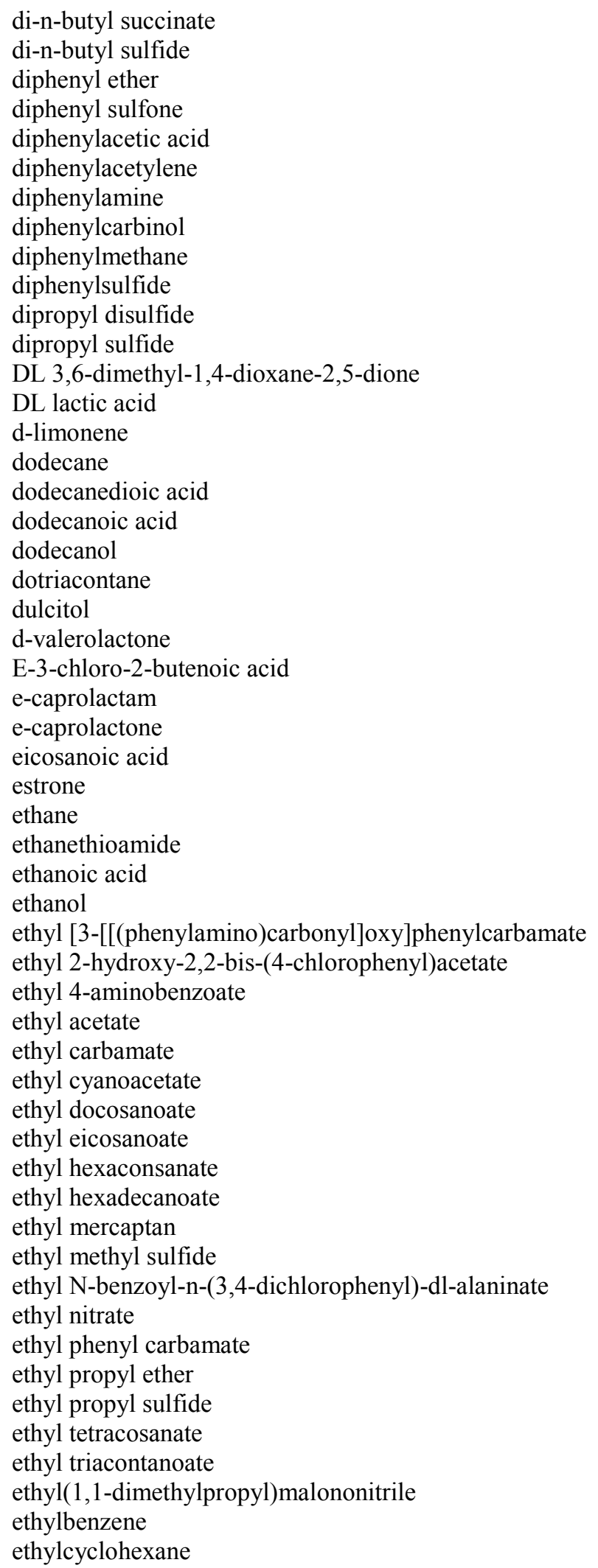

29.21

18.59

17.21

21.78

31.25

20.50

17.86

23.00

18.58

13.98

13.81

12.13

24.70

11.34

11.38

36.82

50.57

36.65

40.17

117.94

65.10

11.29

13.81

16.10

13.82

69.20

48.03

2.79

18.36

11.72

9.12

32.75

23.48

23.56

10.48

15.23

11.78

28.74

23.36

40.27

15.09

4.97

9.76

27.06

8.53

16.27

8.39

10.58

34.14

52.27

19.25

9.16

8.33
33.48

24.03

15.83

19.41

23.78

15.46

21.06

19.03

16.26

15.25

20.12

18.13

17.66

17.31

14.84

33.01

47.60

40.31

39.41

91.99

35.07

16.47

19.94

25.70

18.80

63.90

22.76

3.52

19.29

13.15

9.92

41.39

26.20

21.36

11.99

20.13

16.93

68.64

62.74

80.43

50.94

6.56

9.28

27.59

8.72

22.08

13.94

15.18

86.33

92.23

22.54

10.94

16.28 


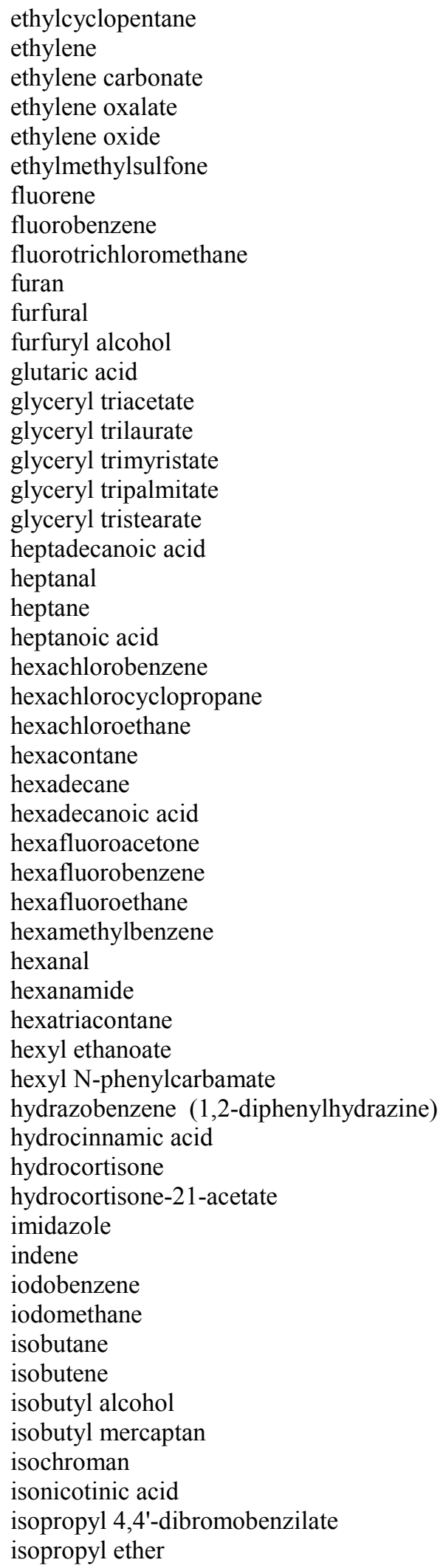

furan

furfural

hexamethylbenzene

indene 


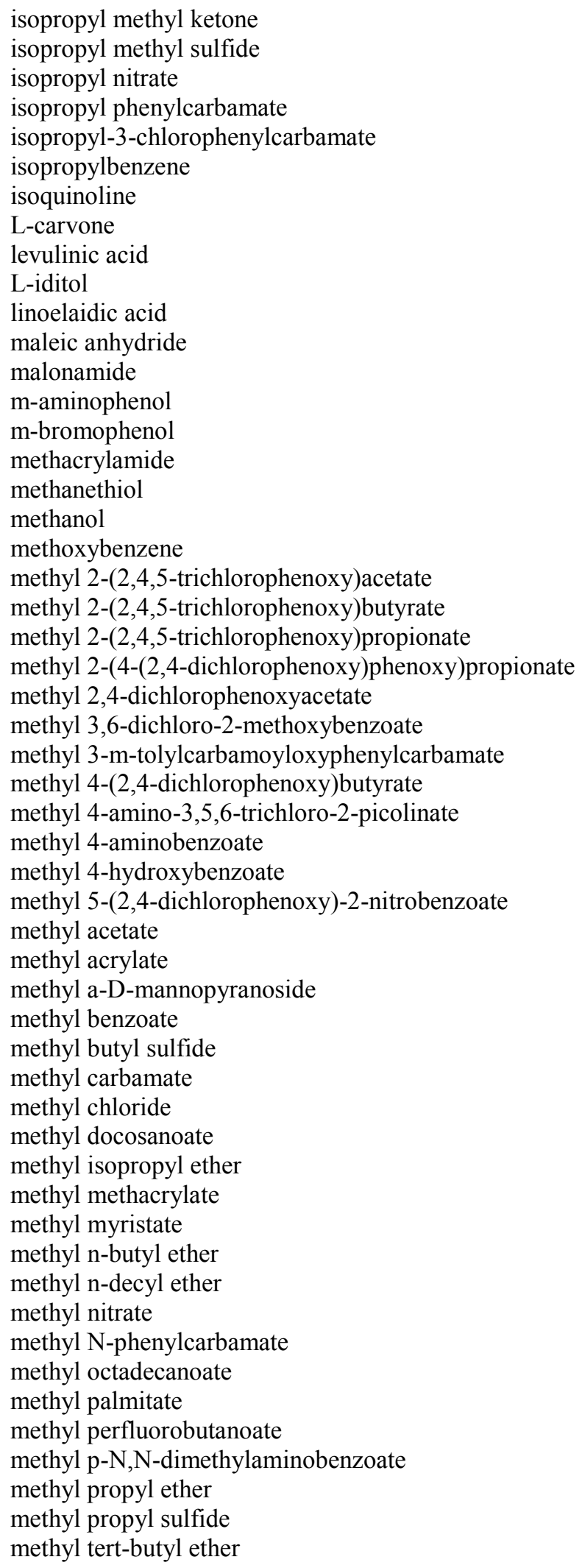

68.16 
methyl tert-butyl sulfide

methyl tetrachloroterephthalic acid ester

16.89

26.12

methyl-3,4-dichlorophenylcarbamate

$23.19 \quad 23.38$

methylamine

$\begin{array}{ll}6.13 & 5.91 \\ 5.76 & 8.67\end{array}$

methylcyclobutane

$\begin{array}{ll}5.76 & 8.67\end{array}$

methylcyclohexane

$6.69 \quad 13.33$

methylcyclopentane

$6.93 \quad 11.00$

methylenecyclobutane

$\begin{array}{ll}5.86 & 8.50\end{array}$

methylhydrazine

methylphenylsulfide

$10.42 \quad 10.72$

methylphosphonyl chlorofluoride

$14.85 \quad 12.94$

methylphosphonyl dichloride

$11.85 \quad 12.54$

$18.08 \quad 13.55$

methylphosphonyl difluoride

$11.88 \quad 10.46$

$10.71 \quad 14.04$

m-nitrobenzoic acid

$19.33 \quad 22.67$

m-nitrophenol

mono(2,2-dimethylhydrazide) butanedioic acid

$19.19 \quad 16.68$

$36.97 \quad 33.25$

$\mathrm{m}$-phenylenediamine

15.40

16.56

m-terphenyl

$22.59 \quad 19.92$

m-toluic acid

15.73

$8.80 \quad 13.84$

m-xylen

11.59

$\begin{array}{ll}47.90 & 30.43\end{array}$

myo-inositol

25.19

N-(1-ethylpropyl)-2,6-dinitro-3,4-xylidine

23.08

32.47

$\mathrm{N}(3)$ N(3)-diethyl-2,4-dinitro-6-(trifluoromethyl)-1,3-benzenediamine

$29.13 \quad 26.70$

$32.04 \quad 22.67$

$\mathrm{N}$-(3,4-dichlorophenyl)-2-methyl-2-propenamide

27.48

29.91

$\mathrm{N}$-(3-chloro-4-methylphenyl)-2-methylpentanamide

16.35

30.14

$\mathrm{N}$-(4-chlorophenyl)-2,2-dimethylpentanamide

$\mathrm{N}$-(4-chlorophenyl)-N-methoxy-N-methylurea

23.31

30.86

22.54

25.31

$\mathrm{N}$-(cyclopropylmethyl)-2,6-dinitro-n-propyl-4-(trifluoromethyl)

benzenamine

22.51

30.25

N,N'-(2-hydroxyethyl)-1,4-diaminoanthraquinone

32.34

50.27

N,N-(2-hydroxyethyl)-4-(4-nitrophenyl)azoaniline

32.43

40.67

N,N-(2-hydroxyethyl)-4-phenylazoaniline

N,N-diethyl-2-(1-naphthyloxy)propionamide

$\mathrm{N}, \mathrm{N}$-dimethyl-1,3-propanediamine

29.96

37.23

24.57

30.52

$12.38 \quad 17.77$

$25.43 \quad 20.90$

$25.43 \quad 25.26$

$\mathrm{N}, \mathrm{N}$-dimethyl-2,2-diphenylbenzeneacetamide

N,N-dimethyl-2-methylcarbamoyloxyimino-2-(methylthio)acetamide

30.17

32.43

N,N-dimethyl-4-phenylazoaniline

23.08

18.54

$\mathrm{N}, \mathrm{N}$-dimethylaniline

$\mathrm{N}, \mathrm{N}$-dimethylformamide

12.75

12.28

$8.95 \quad 11.43$

$13.64 \quad 15.45$

$10.07 \quad 10.80$

$\mathrm{N}, \mathrm{N}$-dimethylhydrazine

$60.32 \quad 25.63$

N,N'-dimethyl-N,N'dinitro-1,2-ethanediamine

61.68

37.43

N,N-dimethyl-N'-[3-(trifluoromethyl)-phenyl]urea

29.82

27.61

N,N-dimethyl-N'-[4-(1-methylethyl)phenyl]urea

33.87

26.77

$\begin{array}{ll}33.879 & 65.38\end{array}$

$\mathrm{N}, \mathrm{N}$-dinitro-diaminomethane

35.85

26.77 
$\mathrm{N}, \mathrm{N}^{\prime}$-dinitroethanediamine

29.50

29.72

$\mathrm{N}, \mathrm{N}$-di-n-propyladipamide

36.11

47.69

$\mathrm{N}-[(1,1,2,2$-tetrachloroethyl)thio]-4-cyclohexene-1,2-dicarboximide

43.10

27.89

$\mathrm{N}$-[4-(methoxymethyl)-1-[2-(2-thienyl)ethyl]-4-piperidinyl]-

N-phenyl-propanamide

23.85

40.61

$\mathrm{N}$-[4-methyl-3-[[(trifluoromethyl)sulfonyl]amino]phenyl]acetamide

30.75

N-[5-(1,1-dimethylethyl)-1,3,4-thiadiazol-2-yl]-N,N'-dimethylurea

40.47

27.95

$\mathrm{N}$-acetyl-D-leucine amide

29.48

33.98

$\mathrm{N}$-acetylglycine amide

20.20

29.40

$\mathrm{N}$-acetylglycyl-L-prolinamide

25.60

39.81

$\mathrm{N}$-acetyl-L-alanine amide

$\mathrm{N}$-acetyl-L-isoleucinamide

32.60

21.70

33.98

$\mathrm{N}$-acetyl-L-prolyl-glycinamide

39.81

$\mathrm{N}$-acetyl-pyrazinamide

41.80

22.39

$\mathrm{N}$-acetylsarcosinamide

23.60

23.53

$\mathrm{N}$-allyl-N-phenylthiourea

27.40

28.44

naphthalene

naphthalene 1,8-disulfide

27.61

13.06

19.10

15.04

naphthalene 1,8-disulfide s-oxide

13.00

21.06

$\mathrm{N}$-benzylaniline

23.30

22.40

N-butyl dodecanamide

16.76

52.15

n-butyl mercaptan

39.00

12.46

N-butyl tetradecanamide

10.46

58.04

n-butylbenzene

45.00

16.84

n-butylcyclohexane

11.22

22.17

n-butylcyclopentane

11.31

19.84

N-butyl-N'-(3,4-dichlorophenyl)-N-methylurea

27.23

35.93

N-butyl-N-ethyl-2,6-dinitro-4-trifluoromethylaniline

36.50

30.38

N-butylurea

23.77

28.18

n-decane

28.70

27.11

$\mathrm{N}$-decyl hexadecanamide

68.00

81.64

n-decyl-a-cyanoacrylate

41.80

38.56

$\mathrm{N}$-decylundecanamide

42.52

66.89

$\mathrm{N}$-dimethylaminosuccinamic acid

36.97

34.31

n-docosane

77.30

62.50

n-dodecylcyclohexane

45.84

45.77

n-dononacontahectane

698.90

563.83

n-eicosane

67.80

56.60

neopentyl-4,4,4-trinitrobutyrate

30.07

22.59

14.96

n-ethyl-a-cyanoacrylate

12.86

22.28

N-ethylurea

14.39

292.52

n-heneicosane

333.00

59.55

n-heptacosane

63.18

77.25

n-heptadecane

87.59

47.76

N-heptylmyristamide

51.13

66.89

n-hexacosane

55.56

74.30

n-hexane

$\mathrm{N}$-hexyl decanamide

91.70

15.32

13.08

52.15

$\mathrm{N}$-hexyl hexadecanamide

37.00

69.84

$\mathrm{N}$-hexyl tetradecanamide

63.94

nicotinic acid

50.00

20.04

$\mathrm{N}$-isopropylcarbazole

27.48

20.00 
$\mathrm{N}$-isopropylurea

nitrobenzene

12.12

12.97

nitroethane

9.85

8.72

nitromethane

9.70

8.10

N-laurylnonanamide

67.08

66.89

N-methyl O-methyl O-2-chloro-4-tert-butylphenyl phosphoramidate

21.98

22.73

$\mathrm{N}$-methyl-2,4,6,N-tetranitroaniline

25.86

27.26

$\mathrm{N}$-methyl-2-chlorophenylcarbamic acid ester

21.81

22.36

$\mathrm{N}$-methylacetamide

9.73

16.14

$\mathrm{N}$-methylcarbazole

17.15

19.05

$\mathrm{N}$-methyldiphenylacetamide

30.23

26.77

N-methyl-N-nitrobutanamine

37.56

20.48

$\mathrm{N}$-methylpyrrole

7.82

11.99

$\mathrm{N}$-methylurea

14.06

19.33

$\mathrm{N}$-myristylheptanamide

54.76

66.89

N-nitro-bis(N,N-cyanomethyl) amine

38.66

24.49

$\mathrm{N}$-nitro-N-methylaminomethane

37.66

11.63

n-nonacosane

95.81

83.15

n-octacosane

100.10

80.20

n-octane

20.74

21.22

n-octylbenzene

29.96

28.64

nonadecane

nonadecanoic acid

61.06

53.66

nonanal

67.38

60.95

29.60

27.10

nonane

21.76

24.17

nonanoic acid

26.28

31.46

nonyl acrylate

23.36

35.37

nonyl phenylcarbamate

28.07

44.92

N-palmitoyl-pyrazinamide

51.82

61.35

n-pentacontane

185.00

145.07

n-pentacosane

83.81

71.35

43.77

n-pentatriacontane

127.49

41.86

N-phenyl-N[1-(2-phenylethyl)-4-piperidinyl]propanamide (fentanyl)

22.51

100.84

n-propylbenzene

9.27

36.16

13.89

10.37

19.22

n-propylcyclohexane

66.06

66.89

$\mathrm{N}$-propylurea

14.63

25.23

33.11

25.45

$\mathrm{N}$-stearylpropanamide

57.87

66.89

$\mathrm{N}$-tert-butylurea

33.23

24.05

n-tetracosane

n-tetratetracontane

86.19

68.40

145.50

75.73

127.38

n-tricosane

36.50

65.45

n-tridecane

29.37

35.96

30.06

$\mathrm{N}$-vinylpyrrolidone

15.28

16.42

O-(2,4-dichlorophenyl) O-methyl-(1-methylethyl) phosphoramidothioate

29.33

24.38

$\mathrm{O}$-(2-chloro-4-nitrophenyl) O,O-dimethyl phosphorothioate

29.08

21.57

31.15

21.18

O-(4-bromo-2,5-dichlorophenyl) O,O-dimethyl phosphorothioate

31.35

22.29

$\mathrm{O}, \mathrm{O}, \mathrm{O}^{\prime}, \mathrm{O}^{\prime}$-tetramethyl O,O'-thiodi-p-phenylene bis(phosphorothioate)

33.03

30.39

O,O-diethyl O-4-nitrophenyl phosphorothioate

15.72

26.44 
O,O-diethyl O-quinoxalin-2-yl phosphothioate

O-6-ethoxycarbonyl-5-methylpyrazolo[1,5-a]pyrimidin-2-yl O,O-diethyl phosphorothioate

octanoic acid

p-chlorotoluene

p-dioxanone 


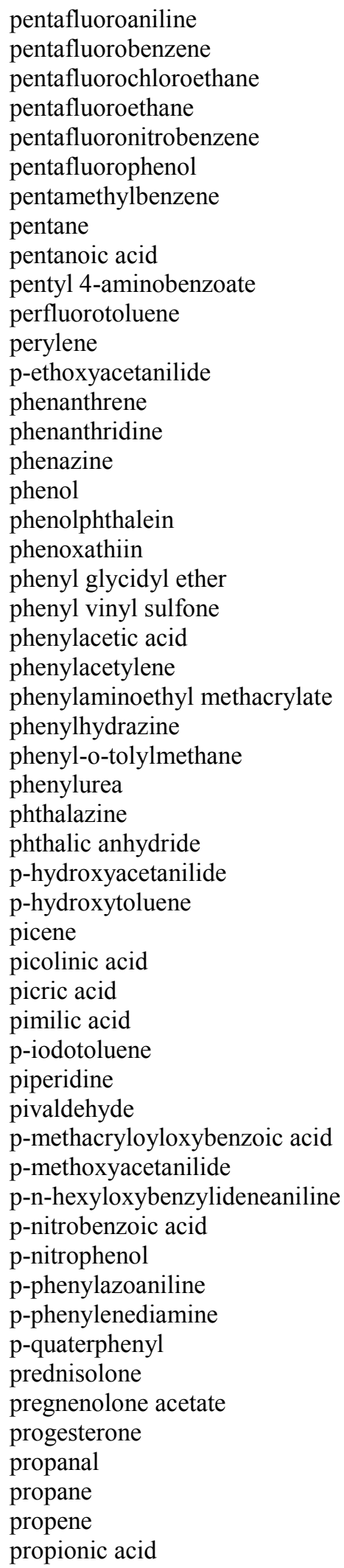

18.21

10.88 


\begin{tabular}{|c|c|c|}
\hline propionitrile & 6.78 & 9.95 \\
\hline propyl 4-aminobenzoate & 20.54 & 24.31 \\
\hline propyl 4-hydroxybenzoate & 27.99 & 24.50 \\
\hline propyl N-phenyl carbamate & 21.08 & 27.22 \\
\hline propylcyclopentane & 10.04 & 16.89 \\
\hline propylene carbonate & 9.62 & 15.79 \\
\hline propylene oxide & 6.57 & 8.33 \\
\hline p-terphenyl & 37.46 & 19.92 \\
\hline p-toluic acid & 22.72 & 20.03 \\
\hline p-toluidine & 17.89 & 13.84 \\
\hline p-xylene & 17.11 & 11.12 \\
\hline pyrazine & 12.95 & 11.14 \\
\hline pyrazole & 14.20 & 16.72 \\
\hline pyrene & 18.38 & 16.95 \\
\hline pyridine & 8.28 & 10.34 \\
\hline pyromellitic dianhydride $1,2,5,6$-benzenetetracarboxylic dianhydride & 15.82 & 23.72 \\
\hline pyrrole & 7.91 & 14.63 \\
\hline pyrrolidine & 9.12 & 12.70 \\
\hline quinazoline & 16.95 & 14.67 \\
\hline quinoline & 10.73 & 13.87 \\
\hline quinoxaline & 11.80 & 14.67 \\
\hline S-(+)-4-isobutyl-a-methylphenyl acetic acid & 18.70 & 22.58 \\
\hline \multicolumn{3}{|l|}{ S-(3,4-dihydro-4-oxobenzo[d]-[1,2,3]-triazin-3-ylmethyl) } \\
\hline O,O-diethyl phoshorodithioate & 25.22 & 30.64 \\
\hline \multicolumn{3}{|l|}{ S-(3,4-dihydro-4-oxobenzo[d][1,2,3]-triazin-3-ylmethyl) } \\
\hline $\mathrm{O}, \mathrm{O}$-dimethyl phoshorodithioate & 27.76 & 24.74 \\
\hline S-2,3,3-trichloroallyl diisopropylthiocarbamate & 27.11 & 21.24 \\
\hline \multicolumn{3}{|l|}{ S-2,3-dihydro-5-methoxy-2-oxo-1,3,4-thiadiazol-3-ylmethyl- } \\
\hline O,O-dimethyl phosphorodithioate & 28.54 & 26.22 \\
\hline sebacic acid & 40.80 & 41.71 \\
\hline spiropentane & 6.43 & 7.04 \\
\hline ß,ß-binaphthyl & 38.90 & 21.79 \\
\hline ß-chloroacetic acid & 12.09 & 15.86 \\
\hline B-chloroacetic acid & 13.93 & 15.86 \\
\hline ß-naphthol & 18.79 & 16.77 \\
\hline ß-naphthyl acetate & 20.05 & 22.61 \\
\hline ß-propiolactone & 9.41 & 11.81 \\
\hline ß-thiolactic acid & 16.97 & 14.00 \\
\hline styrene & 10.96 & 11.05 \\
\hline suberic acid & 28.82 & 35.81 \\
\hline succinic acid & 32.95 & 24.01 \\
\hline succinimide & 17.00 & 14.66 \\
\hline succinonitrile & 12.51 & 16.38 \\
\hline terephthalyl dichloride & 23.44 & 15.54 \\
\hline tert-butyl alcohol & 8.02 & 10.72 \\
\hline tert-butyl amine & 7.29 & 10.01 \\
\hline tert-butyl bromide & 10.07 & 11.14 \\
\hline tert-butyl chloride & 11.17 & 10.33 \\
\hline tert-butyl mercaptan & 12.31 & 8.32 \\
\hline tert-butylbenzene & 8.41 & 12.80 \\
\hline tert-butylmethylsulfone & 24.69 & 17.02 \\
\hline testosterone & 29.38 & 21.04 \\
\hline testosterone acetate & 27.88 & 23.16 \\
\hline
\end{tabular}


testosterone formate

26.36

20.09

testosterone propionate

23.78

testosterone valerate

25.64

29.68

tetra(methylthia)methane

24.57

19.48

tetrachloroethene

19.13

8.68

tetrachloro-o-xylene

11.86

15.23

tetrachloro-p-xylene

22.59

15.23

tetracontane

147.46

115.58

tetracyanoethylene

24.92

18.97

tetradecane

tetradecanoic acid

45.07

38.91

tetrafluoroethylene

45.10

46.21

tetrahydrofuran

7.71

4.65

$8.54 \quad 13.64$

tetramethylsuccinonitrile

30.34

20.09

tetramethylurea

$13.40 \quad 13.88$

tetramethysuccinic acid

22.75

27.72

tetratriacontane

109.24

97.89

tetrazole

17.70

theophylline

28.20

18.41

thiacyclobutane

8.91

thiacyclohexane

13.50

thiacyclopentane

7.35

27.55

thianthrene

thiazole

thiophene

9.58

6.69

11.48

12.55

thiourea

26.10

28.97

13.78

18.44

16.11

19.80

10.35

9.55

thioxanthene 50-195

35.50

11.43

20.01

thioxanthone

thymine

thymol

toluene

tolyl vinyl sulfone

17.51

18.70

21.53

22.01

21.39

15.78

$6.61 \quad 10.33$

$10.88 \quad 16.06$

trans,cis-2,6-octadiene-1,8-dioic acid

22.78

32.49

trans,trans-2,6-octadiene-1,8-dioic acid

41.39

32.49

trans-1-(4-heptanoylphenyl)-4-heptylcyclohexane

24.20

52.28

trans-1,2-cyclohexanediol

18.51

19.46

10.50

12.67

trans-1,3-dimethylcyclohexane

9.87

12.67

trans-1,3-dimethylcyclopentane

7.41

10.34

trans-1,3-pentadiene

7.14

9.21

trans-1,4-dimethylcyclohexane

12.34

12.67

trans-1,4-di-tert-butylcyclohexane

17.15

22.10

trans-10-octadecenoic acid

58.52

49.06

trans-11-octadecenoic acid

58.52

49.06

trans-12-octadecenoic acid

56.71

49.06

trans-13-octadecenoic acid

55.62

49.06

trans-14-octadecenoic acid

57.06

49.06

trans-15-octadecenoic acid

58.98

49.06

trans-1-heptyl-4-(4-nonanoylphenyl)cyclohexane

32.12

58.17

trans-2-butene

9.76

5.14

trans-2-pentene

8.35

5.76

trans-3-octadecenoic acid

57.15

49.91 
trans-4-octadecenoic acid

trans-azobenzene

triacontane

triamcinolone

42.56

33.39

triatriacontane

105.02

94.94

tribromomethane

11.09

8.90

trichloroacetic acid

trichloroethylene

8.45

7.15

trichloromethane

8.80

6.45

tridecandioic acid

45.30

50.55

tridecanoic acid

42.69

43.26

tridecanol

45.41

trifluoroacetonitrile

4.97

42.36

trifluoromethane

4.06

7.98

trifluoromethanethiol

trifluoromethyl (2-hydroxy-1-propenyl)ketone

4.93

0.99

8.45

11.90

trimethylamine

6.54

trimethylhydrazine

9.49

trinitroglycerine

21.87

29.61

triphenyl phosphate

24.89

triphenylamine

27.90

24.74

4.50

15.74

26.33

triphenylchloromethane

21.97

triphenylmethane

24.22

triphenylphosphine oxide

12.73

39.65

undecanedioic acid

undecanoic acid

34.45

15.97

6.54

undecanolactone

12.93

12.61

27.76

25.90

12.59

22.08

20.12

20.96

17.66

24.86

44.66

37.36

30.57

urea

8.46

18.06

vinyl acetate

4.92

7.91

vinyl chloride

19.20

4.11

xanthene

26.12

17.19

xanthone

20.71

20.03

Z-3-chloro-2-butenoic acid

18.64

z-enantholactam 
APPENDIX C. Rotational symmetry number $(\sigma)$, Flexibility count $(\Phi)$, experimental entropy of melting and predicted entropy of melting $\left(\Delta \mathrm{S}_{\mathrm{m}}\right)$ for

1663 organic compounds.

Name

(+)2-butanol

(+)a-(3-benzoylphenyl)propionic acid

(2,4,5-trichlorophenoxy)acetic acid

(2,4-dichlorophenoxy)acetic acid

(4-chloro-2-methylphenoxy)acetic acid

(4-chloro-o-tolyloxy)acetic acid

(d) 1,2-dibromoacenaphthene

(d) 1,2-dichloroacenaphthene

(d) 1,2-diphenyl-1,2-dihydroxyethane

(d) 2-(1-naphthoxy)propionamide

(D) 2-(2-chloro-3-methylphenoxy)propionic acid

(d) 2-(m-chlorophenoxy)propanoic acid

(d) 2-(o-chlorophenoxy)propanoic acid

(d) 2-(p-bromophenoxy)propanoic acid

(d) 2-(p-methoxyphenyl)propiophenone

(d) 2-(p-nitrophenoxy)propanoic acid

(d) 2,3-dibromo-1,4-butanediol

(d) 2-phenoxypropionic acid

(d) 3-(m-bromophenyl)-3-hydroxypropanoic acid

(d) 3-(m-chlorophenyl)-3-hydroxypropanoic acid

(d) 3-(m-fluorophenyl)-3-hydroxypropanoic acid

(d) 3-(o-fluorophenyl)-3-hydroxypropanoic acid

(d) 3-(p-bromophenyl)-3-hydroxypropanoic acid

(d) 3-(p-chlorophenyl)-3-hydroxypropanoic acid

(d) 3-(p-fluorophenyl)-3-hydroxypropanoic acid

(d) 3-hydroxy-3-phenylbutyric acid

(d) 3-hydroxy-3-phenylvaleric acid

(d) 3-phenyl-3-hydroxy-2,2-dimethylpropanoic acid

(d) 3-phenyl-3-hydroxypropanoic acid

(d) dimethyl diacetyltartrate

(d) dimethyl tartrate

(d) malic acid

(d) mandelic acid

(d) methylenebisthiopropionic acid

(d) m-fluoromandelic acid

(d) o-chloromandelic acid

(d) o-fluoromandelic acid

(d) p-chloromandelic acid

(d) p-fluoromandelic acid

(dl) 1,2-dibromoacenaphthene

(dl) 1,2-dichloroacenaphthene

(dl) 1,2-diphenyl-1,2-dihydroxyethane

\begin{tabular}{|c|c|c|c|}
\hline & & $\underline{\Delta} \mathbf{S}_{\mathbf{m}_{-}}$ & .mol) \\
\hline$\sigma$ & $\Phi$ & $\exp$ & pred \\
\hline 1 & 1.0 & 33.8 & 57.4 \\
\hline 1 & 1.0 & 76.8 & 57.4 \\
\hline 1 & 2.0 & 88.1 & 64.8 \\
\hline 1 & 2.0 & 85.6 & 64.8 \\
\hline 1 & 2.0 & 76.3 & 64.8 \\
\hline 1 & 2.0 & 76.3 & 64.8 \\
\hline 2 & 0.0 & 63.4 & 44.3 \\
\hline 2 & 0.0 & 56.9 & 44.3 \\
\hline 1 & 2.0 & 81.6 & 64.8 \\
\hline 1 & 2.0 & 80.1 & 64.8 \\
\hline 1 & 2.0 & 61.7 & 64.8 \\
\hline 1 & 2.0 & 80.8 & 64.8 \\
\hline 1 & 2.0 & 72.6 & 64.8 \\
\hline 1 & 2.0 & 72.7 & 64.8 \\
\hline 1 & 2.0 & 66.7 & 64.8 \\
\hline 1 & 2.0 & 57.8 & 64.8 \\
\hline 1 & 3.0 & 87.3 & 72.1 \\
\hline 1 & 2.0 & 62.9 & 64.8 \\
\hline 1 & 2.0 & 68.1 & 64.8 \\
\hline 1 & 2.0 & 76.2 & 64.8 \\
\hline 1 & 2.0 & 78.0 & 64.8 \\
\hline 1 & 2.0 & 64.9 & 64.8 \\
\hline 1 & 2.0 & 89.3 & 64.8 \\
\hline 1 & 2.0 & 72.8 & 64.8 \\
\hline 1 & 2.0 & 81.3 & 64.8 \\
\hline 1 & 2.0 & 63.3 & 64.8 \\
\hline 1 & 3.0 & 81.7 & 72.1 \\
\hline 1 & 2.0 & 92.2 & 64.8 \\
\hline 1 & 2.0 & 83.5 & 64.8 \\
\hline 1 & 7.0 & 77.7 & 101.7 \\
\hline 1 & 4.0 & 53.9 & 79.5 \\
\hline 1 & 2.0 & 61.2 & 64.8 \\
\hline 1 & 1.0 & 64.9 & 57.4 \\
\hline 1 & 5.0 & 63.6 & 86.9 \\
\hline 1 & 1.0 & 61.6 & 57.4 \\
\hline 1 & 1.0 & 62.9 & 57.4 \\
\hline 1 & 1.0 & 57.6 & 57.4 \\
\hline 1 & 1.0 & 58.4 & 57.4 \\
\hline 1 & 1.0 & 71.7 & 57.4 \\
\hline 2 & 0.0 & 63.2 & 44.3 \\
\hline 2 & 0.0 & 60.5 & 44.3 \\
\hline 1 & 2.0 & 79.8 & 64.8 \\
\hline
\end{tabular}


(dl) 2-(1-naphthoxy)propionamide

(dl) 2-(2-chloro-3-methylphenoxy)propionic acid

(dl) 2-(m-chlorophenoxy)propanoic acid

(dl) 2-(o-chlorophenoxy)propanoic acid

(dl) 2-(p-bromophenoxy)propanoic acid

(dl) 2-(p-methoxyphenyl)propiophenone

(dl) 2-(p-nitrophenoxy)propanoic acid

(dl) 2,3-dibromo-1,4-butanediol

(dl) 2-phenoxypropionic acid

(dl) 3-(m-bromophenyl)-3-hydroxypropanoic acid

(dl) 3-(m-chlorophenyl)-3-hydroxypropanoic acid

(dl) 3-(m-fluorophenyl)-3-hydroxypropanoic acid

(dl) 3-(o-fluorophenyl)-3-hydroxypropanoic acid

(dl) 3-(p-bromophenyl)-3-hydroxypropanoic acid

(dl) 3-(p-chlorophenyl)-3-hydroxypropanoic acid

(dl) 3-(p-fluorophenyl)-3-hydroxypropanoic acid

(dl) 3-hydroxy-3-phenylbutyric acid

(dl) 3-hydroxy-3-phenylvaleric acid

(DL) 3-methylnonane

(dl) 3-phenyl-3-hydroxy-2,2-dimethylpropanoic acid

(dl) 3-phenyl-3-hydroxypropanoic acid

(DL) 4-methylnonane

(dl) dimethyl diacetyltartrate

(dl) dimethyl tartrate

(dl) malic acid I

(dl) malic acid II

(dl) mandelic acid

(dl) menthol

(dl) methylenebisthiopropionic acid

(dl) m-fluoromandelic acid

(dl) o-chloromandelic acid

(dl) o-fluoromandelic acid

(dl) p-chloromandelic acid

(dl) p-fluoromandelic acid

(l) menthol

[(benzoylamino)oxy] acetic acid

1-(4-chlorophenoxy)-3,3-dimethyl-(1H,1,2,4-triazol-1-yl)-

2-butanone

1-(methylamino)-9,10-anthracenedione

1-(o-chlorophenyl)thiourea

1,1-(2,2,2-trichloroethylidene)bis(4-chlorobenzene)

1,1'-(2,2,2-trichloroethylidene-bis(4-methoxy)benzene

1,1'-(2,2-dichloroethylidene)bis(4-ethylbenzene)

1,1'-(2,2-dichloroethylidenebis(4-chlorobenzene)

1,1-(di-p-chlorophenyl)-2-nitropropane

1,1,1,3-tetrachloropropane

1,1,1-trichloro-3,3,3-trifluoropropane

1,1,1-trichloroethane

1,1,1-trifluoro-3,3-dichloropropane

1,1,1-trifluoro-3-chloropropane

1,1,1-trifluoroethane

1,1,1-trifluoro-n-[2-methyl-4-(phenylsulphonyl)phenyl]

methane sulfonamide

$\begin{array}{rrr}2.0 & 84.6 & 64.8 \\ 2.0 & 78.0 & 64.8 \\ 2.0 & 85.6 & 64.8 \\ 2.0 & 83.0 & 64.8 \\ 2.0 & 82.6 & 64.8 \\ 2.0 & 74.7 & 64.8 \\ 2.0 & 78.3 & 64.8 \\ 3.0 & 80.6 & 72.1 \\ 2.0 & 85.2 & 64.8 \\ 2.0 & 76.7 & 64.8 \\ 2.0 & 70.1 & 64.8 \\ 2.0 & 70.7 & 64.8 \\ 2.0 & 79.5 & 64.8 \\ 2.0 & 77.8 & 64.8 \\ 2.0 & 83.2 & 64.8 \\ 2.0 & 76.3 & 64.8 \\ 2.0 & 59.6 & 64.8 \\ 3.0 & 89.2 & 72.1 \\ 6.0 & 99.2 & 94.3 \\ 2.0 & 91.5 & 64.8 \\ 2.0 & 81.2 & 64.8 \\ 6.0 & 86.9 & 94.3 \\ 7.0 & 73.0 & 101.7 \\ 4.0 & 74.8 & 79.5 \\ 2.0 & 83.4 & 64.8 \\ 2.0 & 76.2 & 64.8 \\ 1.0 & 65.1 & 57.4 \\ 3.0 & 34.0 & 72.1 \\ 5.0 & 91.7 & 86.9 \\ 1.0 & 66.7 & 57.4 \\ 1.0 & 56.0 & 57.4 \\ 1.0 & 77.2 & 57.4 \\ 1.0 & 69.0 & 57.4 \\ 1.0 & 72.7 & 57.4 \\ 3.0 & 37.6 & 72.1 \\ 3.5 & 75.5 & 75.8 \\ & & \\ 3.0 & 65.1 & 72.1 \\ 1.0 & 65.0 & 57.4 \\ 1.5 & 53.9 & 61.1 \\ 2.0 & 68.8 & 64.8 \\ 4.0 & 76.2 & 79.5 \\ 4.0 & 70.4 & 79.5 \\ 2.0 & 71.5 & 64.8 \\ 3.0 & 60.4 & 72.1 \\ 1.0 & 53.4 & 57.4 \\ 0.0 & 60.5 & 50.0 \\ 0.0 & 42.2 & 40.9 \\ 1.0 & 56.7 & 57.4 \\ 1.0 & 54.6 & 57.4 \\ 0.0 & 38.2 & 40.9 \\ & 76.0 & 64.8\end{array}$


1,1,1-trinitroethane

1,1,2,2-tetrachlorodifluoroethane

$\begin{array}{lll}0.0 & 49.6 & 50.0 \\ 0.0 & 18.4 & 50.0 \\ 1.0 & 42.1 & 57.4 \\ 0.0 & 37.3 & 44.3 \\ 1.0 & 48.0 & 57.4 \\ 0.0 & 20.5 & 50.0\end{array}$

1,1,2,2-tetrachloroethane

1,1,2-tribromoethane

1,1,2-trichloroethane

1,1,2-trifluoro-1,2,2-trichloroethane

1,1,3,3-tetraethylurea

1,1,3-trimethylurea

1,10-decanediol

1,12-benzoperylene

1,1-bis(4-chlorophenyl)-2-nitrobutane

1,1-dichloro-2,2-bis(4-chlorophenyl)ethylene

1,1-dichloroethane

1,1-dichloroethene

1,1-dicyclohexyldodecane

1,1-diethylurea

1,1-difluoro-1-chloroethane

1,1-dimethyl-3-phenylurea

1,1-dimethylcyclohexane

1,1-dimethylcyclopentane

1,1-dimethylurea

1,1-diphenyldodecane

1,2,3,4,5,6,7,8-octahydroanthracene

1,2,3,4,5-pentahydroxypentane (D-Arabitol)

1,2,3,4,5-pentahydroxypentane (Ribitol)

1,2,3,4,5-pentahydroxypentane (Xylitol)

1,2,3,4-tetracarbomethoxybenzene

1,2,3,4-tetracarbomethoxynaphthalene

1,2,3,4-tetrachlorobenzene

1,2,3,4-tetrafluorobenzene

1,2,3,4-tetrahydronaphthlene

1,2,3,4-tetrahydroquinoline

1,2,3,4-tetrahydroxybutane

1,2,3,4-tetramethylbenzene

1,2,3,5-tetracarbomethoxybenzene

1,2,3,5-tetrachlorobenzene

1,2,3,5-tetrafluorobenzene

1,2,3,5-tetramethylbenzene

1,2,3,6,7,8-hexahydropyrene

1,2,3-tribromopropane

1,2,3-tricarbomethoxybenzene

1,2,3-tricarbomethoxynaphthalene

1,2,3-trichlorobenzene

1,2,3-trihydroxybenzene

1,2,3-trihydroxypropane

1,2,3-trimethylbenzene

$1,2,4,5,8,9$-tribenzopyrene

1,2,4,5-tetrabromobenzene

1,2,4,5-tetracarbomethoxybenzene

1,2,4,5-tetracarbomethoxynaphthalene

1,2,4,5-tetrachloro-3-nitrobenzene

1,2,4,5-tetrachlorobenzene

1,2,4,5-tetrafluorobenzene

$\begin{array}{lll}5.5 & 81.2 & 90.6\end{array}$

$\begin{array}{lll}1.5 & 41.5 & 61.1\end{array}$

$\begin{array}{lll}9.0 & 120.7 & 116.4\end{array}$

$\begin{array}{lll}0.0 & 31.3 & 44.3\end{array}$

$\begin{array}{lll}4.0 & 46.7 & 79.5\end{array}$

$\begin{array}{lll}0.0 & 65.3 & 44.3\end{array}$

$\begin{array}{lll}0.0 & 44.7 & 50.0\end{array}$

$\begin{array}{lll}0.0 & 43.1 & 44.3\end{array}$

$\begin{array}{lll}15.0 & 147.5 & 160.7\end{array}$

$\begin{array}{lll}2.5 & 59.5 & 68.5\end{array}$

$\begin{array}{lll}0.0 & 18.9 & 50.0\end{array}$

$\begin{array}{lll}2.0 & 56.3 & 64.8\end{array}$

$\begin{array}{lll}3.0 & 47.4 & 72.1\end{array}$

$\begin{array}{lll}2.0 & 49.5 & 64.8\end{array}$

$\begin{array}{lll}0.5 & 64.1 & 53.7\end{array}$

$\begin{array}{lll}11.0 & 148.1 & 131.2\end{array}$

$\begin{array}{lll}2.0 & 60.4 & 64.8\end{array}$

$\begin{array}{lll}4.0 & 102.5 & 79.5\end{array}$

$\begin{array}{lll}4.0 & 100.3 & 79.5\end{array}$

$\begin{array}{lll}4.0 & 102.3 & 79.5\end{array}$

$\begin{array}{lll}4.0 & 99.8 & 79.5\end{array}$

$\begin{array}{lll}4.0 & 84.7 & 79.5\end{array}$

$\begin{array}{lll}0.0 & 53.1 & 44.3\end{array}$

$\begin{array}{lll}0.0 & 46.8 & 44.3\end{array}$

$\begin{array}{lll}1.0 & 52.4 & 57.4\end{array}$

$\begin{array}{lll}1.0 & 40.7 & 57.4\end{array}$

$\begin{array}{lll}3.0 & 107.0 & 72.1\end{array}$

$\begin{array}{lll}0.0 & 42.3 & 44.3\end{array}$

$\begin{array}{lll}4.0 & 83.8 & 79.5\end{array}$

$\begin{array}{lll}0.0 & 58.7 & 44.3\end{array}$

$\begin{array}{lll}0.0 & 47.0 & 50.0\end{array}$

$\begin{array}{lll}0.0 & 52.0 & 44.3\end{array}$

$\begin{array}{lll}0.0 & 57.7 & 44.3\end{array}$

$\begin{array}{lll}2.0 & 82.2 & 64.8\end{array}$

$\begin{array}{lll}4.0 & 87.0 & 79.5\end{array}$

$\begin{array}{lll}3.0 & 65.3 & 72.1\end{array}$

$\begin{array}{lll}0.0 & 62.7 & 44.3\end{array}$

$\begin{array}{lll}0.0 & 45.6 & 44.3\end{array}$

$\begin{array}{lll}2.0 & 62.4 & 64.8\end{array}$

$\begin{array}{lll}0.0 & 41.0 & 44.3\end{array}$

$\begin{array}{lll}0.0 & 47.4 & 44.3\end{array}$

$\begin{array}{lll}0.0 & 62.3 & 38.5\end{array}$

$\begin{array}{lll}4.0 & 85.7 & 79.5\end{array}$

$\begin{array}{lll}4.0 & 83.1 & 79.5\end{array}$

$\begin{array}{lll}0.0 & 52.1 & 50.0\end{array}$

$\begin{array}{lll}0.0 & 57.2 & 38.5\end{array}$

$\begin{array}{lll}0.0 & 54.3 & 38.5\end{array}$


1,2,4,5-tetramethylbenzene

1,2,4-triazole

1,2,4-tricarbomethoxynaphthalene

1,2,4-trichloro-5-((4-chlorophenyl)sulfonyl)benzene

1,2,4-trimethylbenzene

1,2,5,6-tetracarbomethoxynaphthalene

1,2,5-tricarbomethoxynaphthalene

1,2,6,7-tetracarbomethoxynaphthalene

1,2,6-tricarbomethoxynaphthalene

1,2,7,8-tetrahydroxyoctane

1,2,7-tricarbomethoxynaphthalene

1,2,8-tricarbomethoxynaphthalene

1,2:3,4-dibenzanthracene

1,2:3,4-dibenzopyrene

1,2:4,5-dibenzopyrene

1,2:5,6-dibenzanthracene

1,2-benzacenaphthene (fluoranthene)

1,2-benzanthracene

1,2-benzofluorene

1,2-benzopyrene

1,2-bromochlorobenzene

1,2-bromoiodobenzene

1,2-butadiene

1,2-chloronitrobenzene

1,2-diamino-2-methylpropane

1,2-diaminopropane

1,2-dibenzoylethane

1,2-dibromo-1,1-difluoroethane

1,2-dibromobenzene

1,2-dibromoethane

1,2-dibromotetrafluoroethane

1,2-dicarbomethoxybenzene

1,2-dicarbomethoxynaphthalene

1,2-dichlorobenzene

1,2-dichloroethane

1,2-dichloropropane

1,2-dichloro-tetrafluoroethane

1,2-dicyanobenzene

1,2-difluoro-2,2-dichloroethane

1,2-difluorobenzene

1,2-dihydro-6-neopentyl-2-oxonicotinic acid

1,2-dihydroxybenzene

1,2-diiodobenzene

1,2'-dinaphthylmethane

1,2-dinitrobenzene

1,2-diphenyl-2-(N-piperidinyl)-1-ethanone

1,2-diphenylethane

1,2-pentadiene

1,3,3-trinitroazetidine

1,3,5,5-tetranitro-1,3-diazacyclohexane

1,3,5,7-tetroxane

1,3,5-tri-a-naphthylbenzene

1,3,5-tricarbomethoxynaphthalene

\begin{tabular}{|c|c|c|c|}
\hline 4 & 0.0 & 59.3 & 38.5 \\
\hline 1 & 0.0 & 40.9 & 50.0 \\
\hline 1 & 3.0 & 81.5 & 72.1 \\
\hline 1 & 0.0 & 68.9 & 50.0 \\
\hline 1 & 0.0 & 57.5 & 50.0 \\
\hline 1 & 4.0 & 89.5 & 79.5 \\
\hline 1 & 3.0 & 70.2 & 72.1 \\
\hline 1 & 4.0 & 84.0 & 79.5 \\
\hline 1 & 3.0 & 86.2 & 72.1 \\
\hline 1 & 7.0 & 104.2 & 101.7 \\
\hline 1 & 3.0 & 84.5 & 72.1 \\
\hline 1 & 3.0 & 67.6 & 72.1 \\
\hline 2 & 0.0 & 46.6 & 44.3 \\
\hline 1 & 0.0 & 49.2 & 50.0 \\
\hline 1 & 0.0 & 58.6 & 50.0 \\
\hline 2 & 0.0 & 57.3 & 44.3 \\
\hline 1 & 0.0 & 48.9 & 50.0 \\
\hline 1 & 0.0 & 49.2 & 50.0 \\
\hline 1 & 0.0 & 48.0 & 50.0 \\
\hline 2 & 0.0 & 42.0 & 44.3 \\
\hline 1 & 0.0 & 47.5 & 50.0 \\
\hline 1 & 0.0 & 49.0 & 50.0 \\
\hline 20 & 0.0 & 50.8 & 25.2 \\
\hline 1 & 0.0 & 61.9 & 50.0 \\
\hline 1 & 1.0 & 73.8 & 57.4 \\
\hline 1 & 1.0 & 78.2 & 57.4 \\
\hline 1 & 2.0 & 94.6 & 64.8 \\
\hline 1 & 1.0 & 40.2 & 57.4 \\
\hline 2 & 0.0 & 45.9 & 44.3 \\
\hline 1 & 1.0 & 46.4 & 57.4 \\
\hline 1 & 1.0 & 43.2 & 57.4 \\
\hline 1 & 2.5 & 61.8 & 68.5 \\
\hline 1 & 2.0 & 77.1 & 64.8 \\
\hline 2 & 0.0 & 50.4 & 44.3 \\
\hline 1 & 1.0 & 37.2 & 57.4 \\
\hline 1 & 1.0 & 37.1 & 57.4 \\
\hline 1 & 0.0 & 39.0 & 50.0 \\
\hline 2 & 0.0 & 48.3 & 44.3 \\
\hline 1 & 0.0 & 50.2 & 50.0 \\
\hline 2 & 0.0 & 48.9 & 44.3 \\
\hline 1 & 1.0 & 41.2 & 57.4 \\
\hline 2 & 0.0 & 58.4 & 44.3 \\
\hline 2 & 0.0 & 47.2 & 44.3 \\
\hline 1 & 1.0 & 82.6 & 57.4 \\
\hline 2 & 0.0 & 57.7 & 44.3 \\
\hline 1 & 4.5 & 97.2 & 83.2 \\
\hline 1 & 2.0 & 78.3 & 64.8 \\
\hline 1 & 0.5 & 55.6 & 53.7 \\
\hline 1 & 1.0 & 80.7 & 57.4 \\
\hline 1 & 3.0 & 68.3 & 72.1 \\
\hline 1 & 5.0 & 58.7 & 86.9 \\
\hline 1 & 3.0 & 89.5 & 72.1 \\
\hline 1 & 3.0 & 64.3 & 72. \\
\hline
\end{tabular}


1,3,5-trichloro-2,4,6-trifluorobenzene

$\begin{array}{lll}0.0 & 59.2 & 35.1 \\ 0.0 & 54.1 & 35.1 \\ 0.0 & 41.6 & 35.1 \\ 3.0 & 78.8 & 72.1 \\ 0.0 & 43.9 & 50.0 \\ 3.0 & 58.5 & 72.1 \\ 3.0 & 45.3 & 72.1 \\ 1.0 & 74.9 & 57.4 \\ 0.0 & 55.1 & 50.0 \\ 3.0 & 83.3 & 72.1 \\ 0.0 & 73.0 & 50.0 \\ 3.0 & 71.4 & 72.1\end{array}$

1,3,5-trichlorobenzene

1,3,5-trimethylbenzene

1,3,5-trinitro-1,3,5-triazacyclohexane

1,3,5-trinitrobenzene

1,3,5-trinitroso-1,3,5-triazacyclohexane

1,3,5-trioxane

1,3,5-triphenylbenzene

1,3,6-trimethyluracil

1,3,7-tricarbomethoxynaphthalene

1,3,7-trichlorodibenzodioxin

1,3,8-tricarbomethoxynaphthalene

1,3-bromochlorobenzene

1,3-bromoiodobenzene

1,3-butadiene

1,3-cyclohexadiene

1,3-dibromobenzene

1,3-dibromopropane

1,3-dibutylurea

1,3-dicarbomethoxybenzene

1,3-dicarbomethoxynaphthalene

1,3-dichlorobenzene

1,3-dicyanopropane

1,3-diethylurea

1,3-difluorobenzene

1,3-dihydroxybenzene

1,3-diiodobenzene

1,3-dimethyluracil

1,3-dimethylurea

1,3-dinitro-1,3-diazacycloheptane

1,3-dinitro-1,3-diazacyclohexane

1,3-dinitro-1,3-diazacyclopentane

1,3-dinitro-5-nitroso-1,3,5-triazacyclohexane

1,3-dinitrobenzene

1,3-dioxolane

1,3-diphenylacetone

1,3-diphenylurea

1,3-dithiane

1,3-nitrochlorobenzene

1,4,5,8-tetracarbomethoxynaphthalene

1,4,5-tricarbomethoxynaphthalene

1,4,6-tricarbomethoxynaphthalene

1,4-[bis[(4-methylphenyl)amino]-9,10-anthracenedione

1,4-bis(diphenylphosphino)butane

1,4-bis(phenylglyoxaloyl)benzene

1,4-bis-[4-(4'-n-butylbiphenyl)]butane

1,4-bromochlorobenzene

1,4-bromoiodobenzene

1,4-cyclohexadiene

1,4-cyclohexanedione

1,4-diamino-2-methoxyanthraquinone

3.0

$71.4 \quad 72.1$

$\begin{array}{lll}0.0 & 48.8 & 50.0\end{array}$

$\begin{array}{lll}0.0 & 43.0 & 50.0\end{array}$

$\begin{array}{lll}0.0 & 48.6 & 44.3\end{array}$

$\begin{array}{lll}1.0 & 26.1 & 57.4\end{array}$

$\begin{array}{lll}0.0 & 49.6 & 44.3\end{array}$

$\begin{array}{lll}2.0 & 61.4 & 64.8\end{array}$

$\begin{array}{llr}7.5 & 78.5 & 105.4\end{array}$

$\begin{array}{lll}2.5 & 74.2 & 68.5\end{array}$

$\begin{array}{lll}2.0 & 80.5 & 64.8\end{array}$

$\begin{array}{lll}0.0 & 50.9 & 44.3\end{array}$

$\begin{array}{lll}2.0 & 51.6 & 64.8\end{array}$

$\begin{array}{lll}3.5 & 37.4 & 75.8\end{array}$

$\begin{array}{lll}0.0 & 46.1 & 44.3\end{array}$

$\begin{array}{lll}0.0 & 52.5 & 44.3\end{array}$

$\begin{array}{lll}0.0 & 51.8 & 44.3\end{array}$

$\begin{array}{lll}0.0 & 36.7 & 50.0\end{array}$

$\begin{array}{lll}1.5 & 36.5 & 61.1\end{array}$

$\begin{array}{lll}3.0 & 66.6 & 72.1\end{array}$

$\begin{array}{lll}3.0 & 54.5 & 72.1\end{array}$

$\begin{array}{lll}2.0 & 62.3 & 64.8\end{array}$

$\begin{array}{lll}3.0 & 58.2 & 72.1\end{array}$

$\begin{array}{lll}0.0 & 47.8 & 50.0\end{array}$

$\begin{array}{lll}2.0 & 56.1 & 64.8\end{array}$

$\begin{array}{lll}2.0 & 65.8 & 64.8\end{array}$

$\begin{array}{lll}2.0 & 67.6 & 64.8\end{array}$

$\begin{array}{lll}3.0 & 46.5 & 72.1\end{array}$

$\begin{array}{lll}0.0 & 61.0 & 50.0\end{array}$

$\begin{array}{lll}4.0 & 75.6 & 79.5\end{array}$

$\begin{array}{lll}3.0 & 65.9 & 72.1\end{array}$

$\begin{array}{lll}3.0 & 73.8 & 72.1\end{array}$

$\begin{array}{lll}2.0 & 74.5 & 64.8\end{array}$

$\begin{array}{lll}10.0 & 111.6 & 123.8\end{array}$

$\begin{array}{lll}4.0 & 76.0 & 79.5\end{array}$

$\begin{array}{lll}10.0 & 81.3 & 123.8\end{array}$

$\begin{array}{lll}0.0 & 55.5 & 44.3\end{array}$

$\begin{array}{lll}0.0 & 52.7 & 44.3\end{array}$

$\begin{array}{lll}0.0 & 29.2 & 44.3\end{array}$

$\begin{array}{lll}2.0 & 49.3 & 64.8\end{array}$

1,4-diaminoanthraquinone

1,4-dibromobenzene

$\begin{array}{lll}1.0 & 68.5 & 57.4\end{array}$

$\begin{array}{lll}0.0 & 50.0 & 44.3\end{array}$

$\begin{array}{lll}0.0 & 55.7 & 38.5\end{array}$


1,4-dicarbomethoxybenzene

1,4-dicarbomethoxynaphthalene

1,4-dichloro-2,5-dimethoxybenzene

1,4-dichlorobenzene

1,4-dihydroxybenzene

1,4-dihydroxybutane

1,4-diiodobenzene

1,4-dimethylnaphthalene

1,4-dinitrobenzene

1,4-dioxane

1,4-dioxane-2,5-dione

1,4-di-tert-butylbenzene

1,4-dithiane

1,4-nitrochlorobenzene

1,4-pentadiene

1,5-cyclooctanedione

1,5-dicarbomethoxynaphthalene

1,5-dichloro-3-oxapentane

1,5-dimethyltetrazole

1,5-dinitro-3-nitroso-1,3,5-triazacycloheptane

1,5-pentanediol

1,6-bis-[4-(4'-ethylbiphenyl)]hexane

1,6-dicarbomethoxynaphthalene

1,6-hexanediol

1,7-diacetoxy-2,4,6-trinitro-2,4,6-triazaheptane

1,7-dicarbomethoxynaphthalene

1,7-heptanediol

1,8-bis-(4-biphenyl)octane

1,8-bis-[4-(4'-ethylbiphenyl)]butane

1,8-bis-[4-(4'-ethylbiphenyl)]octane

1,8-bis-[4-(4'-n-butylbiphenyl)]octane

1,8-dimethylnaphthalene

1,8-octanediol

1,9-nonanediol

10H-phenothiazine

10-nonadecanone

10-octadecynoic acid

11-cyclohexyleicosane

11-heneicosanone

11-n-decylheneicosane

11-octadecynoic acid

11-phenyleicosane

12-octadecynoic acid

12-tricosanone

13-octadecynoic acid

14-octadecynoic acid

16-octadecynoic acid

17-octadecynoic acid

1-8-naphthalic anhydride

1a,2a,3ß,4a,5a,6ß-hexachlorocyclohexane

1 -acetoxynaphthalene

1-acetyl-2-naphthol

1-amino-4-hydroxy-2-phenoxy-9,10-anthracenedione

$\begin{array}{rrr}2.5 & 77.5 & 68.5 \\ 2.0 & 60.0 & 64.8 \\ 1.5 & 68.2 & 61.1 \\ 0.0 & 55.7 & 38.5 \\ 0.0 & 59.5 & 38.5 \\ 3.0 & 63.7 & 72.1 \\ 0.0 & 55.6 & 38.5 \\ 0.0 & 37.9 & 44.3 \\ 0.0 & 63.0 & 38.5 \\ 3.0 & 53.5 & 72.1 \\ 0.0 & 47.4 & 50.0 \\ 0.0 & 65.8 & 50.0 \\ 3.0 & 56.2 & 72.1 \\ 0.0 & 33.4 & 44.3 \\ 1.0 & 49.4 & 57.4 \\ 4.0 & 34.9 & 79.5 \\ 2.0 & 67.3 & 64.8 \\ 4.0 & 37.0 & 79.5 \\ 0.0 & 42.1 & 50.0 \\ 4.0 & 70.2 & 79.5 \\ 4.0 & 63.4 & 79.5 \\ 9.0 & 92.1 & 116.4 \\ 2.0 & 59.4 & 64.8 \\ 5.0 & 79.6 & 86.9 \\ 9.0 & 91.1 & 116.4 \\ 2.0 & 55.1 & 64.8 \\ 6.0 & 72.2 & 94.3 \\ 8.0 & 134.9 & 109.1 \\ 6.0 & 101.3 & 94.3 \\ 10.0 & 122.0 & 123.8 \\ 14.0 & 97.2 & 153.3 \\ 0.0 & 46.9 & 44.3 \\ 7.0 & 108.5 & 101.7 \\ 8.0 & 113.9 & 109.1 \\ 0.0 & 58.8 & 50.0 \\ 16.0 & 202.0 & 168.1 \\ 14.0 & 164.0 & 153.3 \\ 19.0 & 180.4 & 190.3 \\ 18.0 & 226.3 & 182.9 \\ 28.0 & 252.0 & 256.7 \\ 14.0 & 174.9 & 153.3 \\ 18.0 & 220.1 & 182.9 \\ 14.0 & 155.6 & 153.3 \\ 20.0 & 228.0 & 197.6 \\ 14.0 & 172.4 & 153.3 \\ 14.0 & 156.5 & 153.3 \\ 14.0 & 173.2 & 153.3 \\ 15.0 & 159.4 & 160.7 \\ 0.0 & 43.0 & 44.3 \\ 3.0 & 57.2 & 72.1 \\ 1.0 & 63.3 & 57.4 \\ 0.0 & 63.3 & 50.0 \\ 1.0 & 67.2 & 57.4\end{array}$


1-aminoanthraquinone

1-aminopropane

1-benzoyl-2-naphthol

1-bromo-2-chloro-1,1,2-trifluoroethane

1-bromo-2-chloroethane

1-bromobutane

1-bromodocosane

1-bromoheptane

1-bromohexane

1-bromonaphthalene

1-bromononane

1-bromooctane

1-bromopentane

1-bromotricosane

1-bromoundecane

1-butene

1-butyne

1-chloro-2-(2,2,2-trichloro-1-(4-chlorophenyl)ethyl)

benzene

1-chloro-2-(2,2-dichloro-1-(4-chlorophenylethenyl)

benzene

1-chloro-2,2-(bis-(4-chlorophenyl)ethylene

1-chlorodibenzodioxin

1-chloronaphthalene

1-cis-3-pentadiene

1-cyclohexyl-1-phenyldodecane

1-decanethiol

1-decanol

1-decene

1-deoxy-D-glucopyranose

1-docosanol

1H-1,2,4-triazol-3-amine

1-heptanethiol

1-heptanol

1-heptene

1-hexacosanol

1-hexadecanol

1-hexadecene

1-hexanethiol

1-hexanol

1-hexene

1-iodonaphthalene

1-methoxy-2-(2,2,2-tricloro-1-(4-methoxyphenyl)ethyl)

benzene

1-methyl-7-isopropylphenanthrene

1-methyl-9H-pyrido[3,4-b]indole

1-methylcyclohexanol

1-methylcyclopentanol

1-methylnaphthalene

1-methyltetrazole

1-naphthaleneacetamide

1-naphthaleneacetic acid

1-naphthoic acid

$\begin{array}{rrrr}1 & 0.0 & 54.9 & 50.0 \\ 1 & 1.0 & 58.2 & 57.4 \\ 1 & 0.0 & 75.7 & 50.0 \\ 1 & 1.0 & 30.0 & 57.4 \\ 1 & 1.0 & 54.7 & 57.4 \\ 1 & 2.0 & 57.5 & 64.8 \\ 1 & 21.0 & 218.0 & 205.0 \\ 1 & 5.0 & 101.5 & 86.9 \\ 1 & 4.0 & 96.0 & 79.5 \\ 1 & 0.0 & 55.9 & 50.0 \\ 1 & 7.0 & 123.8 & 101.7 \\ 1 & 6.0 & 113.2 & 94.3 \\ 1 & 3.0 & 77.6 & 72.1 \\ 1 & 29.0 & 310.2 & 264.1 \\ 1 & 9.0 & 127.1 & 116.4 \\ 1 & 0.5 & 43.8 & 53.7 \\ 1 & 0.0 & 40.9 & 50.0 \\ & & & \\ 1 & 2.0 & 66.8 & 64.8 \\ & & & \\ 1 & 0.0 & 68.2 & 50.0 \\ 2 & 0.0 & 75.5 & 44.3 \\ 1 & 0.0 & 61.3 & 50.0 \\ 1 & 0.0 & 47.7 & 50.0 \\ 1 & 0.5 & 42.6 & 53.7 \\ 1 & 12.5 & 127.6 & 142.3 \\ 1 & 8.0 & 134.3 & 109.1 \\ 1 & 8.0 & 134.5 & 109.1 \\ 1 & 6.5 & 106.8 & 98.0 \\ 1 & 3.0 & 68.0 & 72.1 \\ 1 & 21.0 & 185.6 & 205.0 \\ 1 & 0.0 & 51.2 & 50.0 \\ 1 & 5.0 & 110.5 & 86.9 \\ 1 & 5.0 & 75.5 & 86.9 \\ 1 & 3.5 & 81.9 & 75.8 \\ 1 & 25.0 & 243.1 & 234.6 \\ 1 & 15.0 & 181.2 & 160.7 \\ 1 & 13.0 & 124.4 & 146.0 \\ 1 & 4.0 & 93.6 & 79.5 \\ 1 & 4.0 & 68.6 & 79.5 \\ 1 & 2.5 & 70.1 & 68.5 \\ 1 & 0.0 & 56.8 & 50.0 \\ & & & \\ 1 & 4.0 & 64.6 & 79.5 \\ 1 & 1.0 & 48.9 & 57.4 \\ 1 & 0.0 & 53.3 & 50.0 \\ 1 & 3.0 & 36.3 & 72.1 \\ 1 & 2.0 & 27.1 & 64.8 \\ 1 & 0.0 & 49.3 & 50.0 \\ 1 & 0.0 & 49.8 & 50.0 \\ 1 & 1.0 & 72.1 & 57.4 \\ 1 & 1.0 & 54.9 & 57.4 \\ 1 & 0.0 & 45.7 & 50.0\end{array}$


1-naphthyl benzoate

1-naphthyl methylcarbamate

1-naphthylamine

1-nitronaphthalene

1-nonanethiol

1-nonene

1-octanethiol

1-octene

1-pentadecanol

1-pentanethiol

1-pentanol

1-pentene

1-propanethiol

1-propanol

1-tetradecanol

1 -undecene

2-((4-chloro-6-(cyclopropylamino)-1,3,5-triazin-2-yl)

amino-2-methylpropanenitrile

2-(1,3-dioxolan-2-yl)phenyl methylcarbamate

2-(1'-cyclohexenyl)cyclohexanone

2-(1-methylethyl)phenyl methylcarbamate

2-(2,4,5-trichlorophenoxy)propanoic acid

2-(2,4-dichlorophenoxy)propanoic acid

2-(3,4-dichlorophenyl)-4-methyl-1,2,4-oxadiazolidine-

3,5-dione

2-(3-hydroxy-2-quinolinyl)-1H-indene-1,3(2H)-dione

2-(4-chloro-2-methylphenoxy)propanoic acid

2-(6-methoxy-2-naphthyl)propionic acid

2-(dimethylamino)-1,2-diphenylethanone

2-(docosanoxy)ethanol

2-(hexadecyloxy)ethanol

2,11-dicyclohexyldodecane

2,2,2-trinitroethanol

2,2,2-trinitroethyl 4,4,4-trinitrobutyrate

2,2,2-trinitroethyl-4,4-dinitropentanoate

2,2',3,3',4,5,5',6,6'-nonachlorobiphenyl

$2,2^{\prime}, 3,3^{\prime}, 5,5^{\prime}, 6,6^{\prime}$-octachlorobiphenyl

2,2',3,3',5,5',6-heptachlorobiphenyl

2,2',3,3',5,5'-hexachlorobiphenyl

2,2',3,3',6,6'-hexachlorobiphenyl

2,2,3,3-tetramethylbutane

2,2,3,3-tetramethylpentane

2,2,3-trimethylbutane

$2,2^{\prime}, 4,4$ ',6,6'-hexachlorobiphenyl

2,2,4,4-tetramethylpentan-3-ol

2,2,4,4-tetramethylpentane

2,2',4,5,5'-pentachlorobiphenyl

2,2',4',5-tetrachlorobiphenyl

2,2,4-trimethylpentane

2,2,5,5-tetramethylhex-3-ene

2,2,6,6-tetramethyl-1,3-dioxane

2,2-bis-(4-cyanatophenyl)propane

2,2-bis(phenylthio)propane

$\begin{array}{rrr}1.0 & 51.6 & 57.4 \\ 2.0 & 58.9 & 64.8 \\ 0.0 & 48.1 & 50.0 \\ 0.0 & 55.9 & 50.0 \\ 7.0 & 125.1 & 101.7 \\ 5.5 & 104.2 & 90.6 \\ 6.0 & 108.3 & 94.3 \\ 4.5 & 89.3 & 83.2 \\ 14.0 & 172.8 & 153.3 \\ 3.0 & 88.8 & 72.1 \\ 3.0 & 53.7 & 72.1 \\ 1.5 & 53.8 & 61.1 \\ 1.0 & 62.2 & 57.4 \\ 1.0 & 36.1 & 57.4 \\ 13.0 & 158.6 & 146.0 \\ 7.5 & 118.2 & 105.4\end{array}$

$\begin{array}{lll}2.5 & 51.3 \quad 68.5\end{array}$

$\begin{array}{lll}2.5 & 61.5 & 68.5\end{array}$

$\begin{array}{lll}7.5 & 61.9 & 105.4\end{array}$

$\begin{array}{lll}3.0 & 70.8 & 72.1\end{array}$

$\begin{array}{lll}2.0 & 87.8 & 64.8\end{array}$

$\begin{array}{lll}2.0 & 78.2 \quad 64.8\end{array}$

$\begin{array}{lll}1.0 & 74.4 & 57.4\end{array}$

$\begin{array}{llll}1 & 0.0 & 57.3 & 50.0\end{array}$

$\begin{array}{llll}1 & 2.0 & 72.2 & 64.8\end{array}$

$\begin{array}{llll}1 & 2.0 & 67.0 & 64.8\end{array}$

$\begin{array}{rrrr}1 & 2.0 & 67.0 & 64.8\end{array}$

$\begin{array}{llll}1 & 24.0 & 171.5 & 227.2\end{array}$

$\begin{array}{llll}1 & 18.0 & 165.1 & 182.9\end{array}$

$\begin{array}{llll}1 & 13.0 & 146.2 & 146.0\end{array}$

$\begin{array}{llll}1 & 1.0 & 65.5 & 57.4\end{array}$

$\begin{array}{llll}1 & 4.5 & 89.1 & 83.2\end{array}$

$\begin{array}{llll}1 & 6.5 & 73.0 & 98.0\end{array}$

$\begin{array}{llll}2 & 0.0 & 49.6 & 44.3\end{array}$

$\begin{array}{llll}4 & 0.0 & 52.6 & 38.5\end{array}$

$\begin{array}{llll}1 & 0.0 & 51.3 & 50.0\end{array}$

$\begin{array}{llll}2 & 0.0 & 68.7 & 44.3\end{array}$

$\begin{array}{llll}1 & 0.0 & 54.8 & 50.0\end{array}$

$\begin{array}{llll}6 & 0.0 & 33.3 & 35.1\end{array}$

$\begin{array}{llll}1 & 1.0 & 50.9 & 57.4\end{array}$

$\begin{array}{llll}1 & 0.0 & 28.5 & 50.0\end{array}$

$\begin{array}{llll}4 & 0.0 & 45.3 & 38.5\end{array}$

$\begin{array}{llll}1 & 0.0 & 29.9 & 50.0\end{array}$

$\begin{array}{llll}1 & 0.0 & 47.2 & 50.0\end{array}$

$\begin{array}{llll}1 & 0.0 & 53.7 & 50.0\end{array}$

$\begin{array}{llll}1 & 0.0 & 69.0 & 50.0\end{array}$

$\begin{array}{llll}1 & 1.0 & 55.5 & 57.4\end{array}$

$\begin{array}{llll}1 & 0.0 & 61.0 & 50.0\end{array}$

$\begin{array}{llll}1 & 3.0 & 43.5 & 72.1\end{array}$

$\begin{array}{llll}1 & 3.0 & 75.0 & 72.1\end{array}$

$\begin{array}{lllll}1 & 1 & 3.0 & 74.2 & 72.1\end{array}$


2,2-bis-hydroxymethylpropanoic acid

2,2-dichloropropane

2,2-dicyanopropane

2,2-dimethoxy-1,2-diphenylethanone

2,2-dimethyl-1,3-dioxan

2,2-dimethyl-1,3-propanediol

2,2-dimethyl-1-propanol

2,2-dimethylbutane

2,2-dimethylheptane

2,2-dimethylpentane

2,2-dimethylpropane

2,2-dimethylpropanoic acid (pivalic acid)

2,2-dinitro-1,3-propanediol

2,2-dinitropropane

2,2-dinitropropanol

2,2-dinitropropyl-4,4-dinitropentanoate

2,2-dintropropyl-4,4,4-trinitrobutyrate

2,2'-methylenebis(3,4,6-trichlorophenol)

2,3,4,5,6-pentachlorobiphenyl

2,3,4,5,6-pentafluorotoluene

2,3,4,5-tetrachlorobiphenyl

2,3,4-trimethylpentane

2,3,5,6-tetrachloro-2,5-cyclohexadiene-1,4-dione

2,3,5-tricarbomethoxynaphthalene

2,3,5-triiodobenzoic acid

2,3,6,7,10,11-hexakis(1-decynyl)triphenylene

2,3,6,7-tetracarbomethoxynaphthalene

2,3,6-tricarbomethoxynaphthalene

2,3,6-trichlorobenzoic acid

2,3,6-trichlorophenylacetic acid

2,3-benzofluorene

2,3-dicarbomethoxynaphthalene

2,3-dichloro-1,4-naphthalenedione

2,3-dichlorophenol

2,3-dihydro-2,2-dimethyl-7-benzofuranol-3-one

$\begin{array}{lll}2.5 & 98.1 & 68.5 \\ 0.0 & 41.4 & 50.0 \\ 0.0 & 45.3 & 50.0 \\ 3.0 & 61.6 & 72.1 \\ 3.0 & 52.7 & 72.1 \\ 1.0 & 55.2 & 57.4 \\ 0.0 & 31.1 & 50.0 \\ 0.0 & 45.9 & 50.0 \\ 3.0 & 76.1 & 72.1\end{array}$

$76.1 \quad 72.1$

$\begin{array}{lll}1.0 & 39.6 & 57.4\end{array}$

$\begin{array}{lll}0.0 & 31.1 & 29.4\end{array}$

$\begin{array}{lll}0.0 & 36.7 & 50.0\end{array}$

$\begin{array}{lll}2.0 & 62.5 & 64.8\end{array}$

$\begin{array}{lll}0.0 & 57.2 & 50.0\end{array}$

$\begin{array}{lll}1.0 & 61.2 & 57.4\end{array}$

$\begin{array}{lll}6.5 & 86.5 & 98.0\end{array}$

$\begin{array}{lll}5.5 & 171.8 & 90.6\end{array}$

$\begin{array}{lll}1.0 & 76.0 & 57.4\end{array}$

$\begin{array}{lll}0.0 & 53.7 & 44.3\end{array}$

$\begin{array}{lll}0.0 & 54.5 & 44.3\end{array}$

$\begin{array}{lll}0.0 & 69.2 & 50.0\end{array}$

$\begin{array}{lll}2.0 & 56.7 & 64.8\end{array}$

$\begin{array}{lll}0.0 & 54.4 & 38.5\end{array}$

$\begin{array}{lll}3.0 & 102.1 & 72.1\end{array}$

$\begin{array}{lll}0.0 & 64.0 & 50.0\end{array}$

$\begin{array}{lll}42.0 & 200.5 & 360.0\end{array}$

$\begin{array}{lll}4.0 & 92.1 & 79.5\end{array}$

$\begin{array}{lll}3.0 & 86.2 & 72.1\end{array}$

$\begin{array}{lll}0.0 & 59.2 & 50.0\end{array}$

$\begin{array}{lll}1.0 & 51.9 & 57.4\end{array}$

$\begin{array}{lll}0.0 & 47.8 & 50.0\end{array}$

$\begin{array}{lll}2.0 & 62.3 & 64.8\end{array}$

$\begin{array}{lll}0.0 & 60.8 & 44.3\end{array}$

$\begin{array}{lll}0.0 & 64.7 & 50.0\end{array}$

2,3-dihydro-2,2-dimethylbenzofuran-7-yl

methylcarbamate

$0.0 \quad 49.5 \quad 50.0$

2,3-dihydro-6-methyl-5-phenylcarbamoyl-1,4-oxathiin-

4,4-dioxide

2,3-dimethyl-2,3-bis(4-tert-butylphenyl)butane

2,3-dimethyl-2,3-bis(phenylazo)butane

2,3-dimethyl-2,3-butanediol

2,3-dimethyl-2,3-diphenylbutane

2,3-dimethyl-2-butene

2,3-dimethylbenzoic acid

2,3-dimethylbutane

2,3-dimethylnaphthalene

2,3-dimethylphenol

2,3-dimethylpyridine

2,3-dinitrophenol

2,3-dinitrotoluene

2,3-pentadiene

2,4,4-trimethyl-1-pentene

$\begin{array}{lll}2.0 & 71.2 & 64.8\end{array}$

$\begin{array}{lll}2.5 & 66.4 & 68.5\end{array}$

$\begin{array}{lll}2.0 & 89.1 & 64.8\end{array}$

$\begin{array}{lll}2.0 & 61.6 & 64.8\end{array}$

$\begin{array}{lll}1.0 & 46.5 & 57.4\end{array}$

$\begin{array}{lll}2.0 & 65.1 & 64.8\end{array}$

$\begin{array}{lll}0.0 & 50.1 & 38.5\end{array}$

$\begin{array}{lll}0.0 & 43.8 & 50.0\end{array}$

$\begin{array}{lll}1.0 & 53.0 & 57.4\end{array}$

$\begin{array}{lll}0.0 & 42.1 & 44.3\end{array}$

$\begin{array}{lll}0.0 & 60.8 & 50.0\end{array}$

$\begin{array}{lll}0.0 & 52.1 & 50.0\end{array}$

$\begin{array}{lll}0.0 & 62.9 & 50.0\end{array}$

$\begin{array}{lll}0.0 & 53.3 & 50.0\end{array}$

$\begin{array}{llll}1 & 0.0 & 44.8 & 25.2 \\ 1 & 0.5 & 49.1 & 53.7\end{array}$


2,4,4-trimethyl-2-pentene

2,4,5,6-tetrachloro-1,3-benzenedicarbonitile

2,4,5-tribromostyrene

2,4,5-trichlorobiphenyl

2,4,5-trichlorophenol

2,4,5-trimethylthiazole

2,4,5-trinitrotoluene

2,4,6,N-tetranitro-N-methyltoluidene

2,4,6-N-tetranitroethylaniline

2,4,6-tribromophenol

2,4,6-trichlorobiphenyl

2,4,6-trimethyl-1,3,5-trioxane

2,4,6-trimethylpyridine

2,4,6-trinitro-1,3-dimethylbenzene

2,4,6-trinitroresorcinol

2,4,6-trinitrotoluene

2,4,6-tri-tert-butylphenol

2,4,7-trinitrofluoren-9-one

2,4-dibromophenol

2,4-dichlorophenol

2,4-dichlorophenyl 4-nitrophenyl ether

2,4-dimethylpentane

2,4-dimethylpyridine

2,4-dimethylpyrrole

2,4-dimethylthiazole

2,4-dinitrochlorobenzene

2,4-dinitrophenol

2,4-dinitrotoluene

2,4-di-tert-butylthiazole

2,4-hexadiyne

2,5,8,11-tetraoxadodecane

2,5,8-trioxanonane

2,5-dibutoxy-1,4-benzoquinone

2,5-dichlorophenol

2,5-diethoxy-1,4-benzoquinone

2,5-dimethylaniline

2,5-dimethylphenol

2,5-dimethylpyridine

2,5-dimethylpyrrole

2,5-dimethyltetrazole

2,5-dimethylthiophene

2,5-di-n-heptadecyloxy-1,4-benzoquinone

2,5-di-n-heptyloxy-1,4-benzoquinone

2,5-di-n-hexadecyloxy-1,4-benzoquinone

2,5-di-n-hexyloxy-1,4-benzoquinone

2,5-dinitrophenol

2,5-di-n-nonadecyloxy-1,4-benzoquinone

2,5-di-n-nonyloxy-1,4-benzoquinone

2,5-di-n-octyloxy-1,4-benzoquinone

2,5-di-n-pentadecyloxy-1,4-benzoquinone

2,5-di-n-undecyloxy-1,4-benzoquinone

2,5-dipentoxy-1,4-benzoquinone

2,5-dipropoxy-1,4-benzoquinone

\begin{tabular}{|c|c|c|}
\hline 0.0 & 40.8 & 50.0 \\
\hline 0.0 & 57.0 & 44.3 \\
\hline 0.0 & 73.8 & 50.0 \\
\hline 0.0 & 65.2 & 50.0 \\
\hline 0.0 & 63.4 & 50.0 \\
\hline 0.0 & 37.4 & 50.0 \\
\hline 0.0 & 65.7 & 50.0 \\
\hline 0.5 & 51.5 & 53.7 \\
\hline 1.5 & 63.7 & 61.1 \\
\hline 0.0 & 50.6 & 50.0 \\
\hline 0.0 & 49.4 & 44.3 \\
\hline 0.0 & 54.4 & 50.0 \\
\hline 0.0 & 41.7 & 50.0 \\
\hline 0.0 & 84.5 & 50.0 \\
\hline 0.0 & 73.7 & 50.0 \\
\hline 0.0 & 66.5 & 44.3 \\
\hline 0.0 & 48.0 & 50.0 \\
\hline 3.0 & 58.8 & 72.1 \\
\hline 0.0 & 46.8 & 50.0 \\
\hline 0.0 & 63.2 & 50.0 \\
\hline 1.0 & 67.1 & 57.4 \\
\hline 2.0 & 44.5 & 64.8 \\
\hline 0.0 & 42.1 & 50.0 \\
\hline 0.0 & 35.8 & 50.0 \\
\hline 0.0 & 13.0 & 44.3 \\
\hline 0.0 & 62.0 & 50.0 \\
\hline 0.0 & 62.3 & 50.0 \\
\hline 0.0 & 58.6 & 50.0 \\
\hline 0.0 & 40.7 & 50.0 \\
\hline 0.0 & 8.5 & 25.2 \\
\hline 9.0 & 103.4 & 116.4 \\
\hline 6.0 & 85.0 & 94.3 \\
\hline 7.5 & 87.2 & 105.4 \\
\hline 0.0 & 67.8 & 50.0 \\
\hline 3.5 & 62.5 & 75.8 \\
\hline 0.0 & 49.1 & 50.0 \\
\hline 0.0 & 67.2 & 50.0 \\
\hline 0.0 & 56.5 & 50.0 \\
\hline 0.0 & 33.1 & 44.3 \\
\hline 0.0 & 52.7 & 50.0 \\
\hline 0.0 & 42.3 & 44.3 \\
\hline 34.0 & 339.7 & 301.0 \\
\hline 14.0 & 154.0 & 153.3 \\
\hline 32.0 & 316.1 & 286.2 \\
\hline 11.5 & 110.3 & 134.9 \\
\hline 0.0 & 62.3 & 50.0 \\
\hline 38.0 & 380.2 & 330.5 \\
\hline 18.0 & 202.7 & 182.9 \\
\hline 16.0 & 132.2 & 168.1 \\
\hline 30.0 & 328.5 & 271.5 \\
\hline 22.0 & 239.1 & 212.4 \\
\hline 9.5 & 115.0 & 120.1 \\
\hline 5.5 & 97.0 & 90.6 \\
\hline
\end{tabular}


2,6-dichloro-4-benzenamine

2

2,6-dichloro-4-nitroaniline

2,6-dichlorobenzonitrile

2,6-dichlorobiphenyl

2,6-dichlorophenol

2,6-diisopropylphenol

2,6-dimethylnaphthalene

2,6-dimethylphenol

2,6-dimethylpyridine

2,6-dinitrochlorobenzene

2,6-dinitro-N,N-dipropyl-4-(trifluoromethyl)benzenamine

2,6-dinitrophenol

2,6-dinitrotoluene

2,6-di-tert-butyl-4-methoxyphenol

2,6-di-tert-butyl-4-methylphenol

2,6-di-tert-butylphenol

2,7-dicarbomethoxynaphthalene

2,7-dimethylnaphthalene

2-[(trichloromethyl)thio]-1H-isoindole-1,3(2H)-dione

2-[[3-(trifluoromethyl)phenyl]amino]-3-

pyridinecarboxylic acid

2-[[4-chloro-6-(ethylamino)-1,3,5-triazin-2-yl]amino]-

2-methylpropanenitrile

2-[bis(2-chloroethyl)amino]tetrahydro-2H-1,3,2-

oxazophosphorine-2-oxide

2-acetoxynaphthalene

2-acetyl-1-naphthol

2-acetylamino-9-[(2-hydroxyethoxy)methyl]-

1,9-dihydro-6H-purin-6-one

2-acetylamino-9-[(2-hydroxyethoxy)methyl]-9H-purine

2-amino-2-hydroxymethylpropane-1,3-diol

2-amino-2-methylpropane-1,3-diol

2-amino-9-[(2-acetoxyethoxy)methyl]-

1,9-dihydro-6H-purin-6-one

2-amino-9-[(2-hydroxyethoxy)methyl]-

1,9-dihydro-6H-purin-6-one

2-amino-9-[(2-hydroxyethoxy)methyl]-9H-purine

2-aminobenzoic acid

2-aminobiphenyl

2-aminopropane

2-benzoyl-1-naphthol

2-bromo-2-chloro-1,1,1-trifluoroethane

2-bromobutane

2-bromonaphthalene

2-bromopropane

2-bromothiophene

2-butanethiol

2-butanol

2-butanone

2-butyne

2-carbomethoxynaphthalene

2-chloro-1-(3-ethoxy-4-nitrophenoxy)-

4-(trifluoromethyl)benzene $\begin{array}{lll}0.0 & 63.1 & 44.3\end{array}$

$\begin{array}{lll}0.0 & 69.9 & 44.3\end{array}$

$\begin{array}{lll}0.0 & 62.7 & 44.3\end{array}$

$\begin{array}{lll}0.0 & 40.9 & 44.3\end{array}$

$\begin{array}{lll}0.0 & 65.1 & 44.3\end{array}$

$\begin{array}{lll}1.5 & 50.0 & 61.1\end{array}$

$\begin{array}{lll}0.0 & 65.4 & 44.3\end{array}$

$\begin{array}{lll}0.0 & 59.3 & 44.3\end{array}$

$\begin{array}{lll}0.0 & 48.8 & 44.3\end{array}$

$\begin{array}{lll}0.0 & 52.5 & 50.0\end{array}$

$\begin{array}{lll}8.0 & 69.4 & 109.1\end{array}$

$\begin{array}{lll}0.0 & 58.3 & 50.0\end{array}$

$\begin{array}{lll}0.0 & 72.8 & 50.0\end{array}$

$\begin{array}{lll}0.5 & 71.8 & 53.7\end{array}$

$\begin{array}{lll}0.0 & 69.4 & 50.0\end{array}$

$\begin{array}{lll}0.0 & 53.3 & 50.0\end{array}$

$\begin{array}{lll}2.0 & 64.8 & 64.8\end{array}$

$\begin{array}{lll}0.0 & 63.3 & 44.3\end{array}$

$\begin{array}{lll}0.5 & 78.1 & 53.7\end{array}$

$\begin{array}{llll}1 & 2.0 & 79.8 & 64.8\end{array}$

$\begin{array}{llll}1 & 3.5 & 95.8 & 75.8\end{array}$

$\begin{array}{llll}1 & 6.5 & 102.7 & 98.0\end{array}$

$\begin{array}{llll}1 & 1.0 & 58.6 & 57.4\end{array}$

$\begin{array}{llll}1 & 0.0 & 60.6 & 50.0\end{array}$

$\begin{array}{llll}1 & 5.0 & 109.8 & 86.9\end{array}$

$\begin{array}{llll}1 & 5.0 & 120.9 & 86.9\end{array}$

$\begin{array}{llll}1 & 3.0 & 87.5 & 72.1\end{array}$

$\begin{array}{llll}1 & 2.0 & 79.4 & 64.8\end{array}$

$\begin{array}{llll}1 & 4.0 & 96.9 & 79.5\end{array}$

$\begin{array}{lll}3.5 & 57.6 & 75.8\end{array}$

$\begin{array}{llll}1 & 3.5 & 91.3 & 75.8\end{array}$

$\begin{array}{llll}1 & 0.0 & 49.1 & 50.0\end{array}$

$\begin{array}{llll}1 & 0.0 & 43.4 & 50.0\end{array}$

$\begin{array}{llll}1 & 0.0 & 41.2 & 50.0\end{array}$

$\begin{array}{llll}1 & 0.0 & 58.7 & 50.0\end{array}$

$\begin{array}{llll}1 & 0.0 & 31.3 & 50.0\end{array}$

$\begin{array}{llll}1 & 1.0 & 42.9 & 57.4\end{array}$

$\begin{array}{llll}1 & 0.0 & 61.9 & 50.0\end{array}$

$\begin{array}{llll}1 & 0.0 & 35.6 & 50.0\end{array}$

$\begin{array}{llll}1 & 0.0 & 38.8 & 50.0\end{array}$

$\begin{array}{llll}1 & 1.0 & 48.7 & 57.4\end{array}$

$\begin{array}{llll}1 & 1.0 & 32.3 & 57.4\end{array}$

$\begin{array}{llll}1 & 0.5 & 45.3 & 53.7\end{array}$

$\begin{array}{llll}1 & 0.0 & 38.4 & 25.2\end{array}$

$\begin{array}{llll}1 & 1.0 & 77.4 & 57.4\end{array}$

$\begin{array}{llll}1 & 5.0 & 83.8 & 86.9\end{array}$ 
2-chloro-2-nitropropane

2-chloro-6-(trichloromethyl)pyridine

$\begin{array}{lll}0.0 & 49.7 & 50.0 \\ 0.0 & 60.1 & 50.0 \\ & & \\ 3.0 & 75.1 & 72.1 \\ 0.0 & 80.7 & 50.0 \\ 0.0 & 62.2 & 50.0 \\ 0.0 & 47.7 & 50.0 \\ 0.0 & 63.8 & 50.0 \\ 1.0 & 42.5 & 57.4\end{array}$

2-chloro-9-(3-dimethylaminopropylidene)-

10-thioxanthene

2-chloroanthraquinone

2-chlorobenzoic acid

2-chlorobiphenyl

2-chlorodibenzodioxin

2-chloroethylphosphonic acid

2-chloro-n-(2,6-diethylphenyl)-n-(methoxymethyl)

acetamide

2-chloronaphthalene

2-chloro-N-isopropyl N-phenylacetamide

2-chloro-N-isopropylacetamide

2-chlorophenol

2-chloropropane

2-chlorothiophene

2-cyano-2-methylpropane

2-cyclohexyl-4,6-dinitrophenol

2-cyclohexylcyclohexanone

2-deoxy-2-fluoro-D-glucopyranose

2-deoxy-D-glucopyranose

2-ethoxyisonitrosoacetanilide

2-ethyl-2-diphenylmethyl-1,3-cyclopentanedione

2-fluorenyl-2-methyl-1,3-cyclohexanedione

2-fluorenyl-2-methyl-1,3-cyclopentanedione

2-fluorotoluene

2-heneicosanone

2-heptanone

6.0

80.1

94.3

0.0

44.3

50.0

$\begin{array}{lll}3.0 & 74.1 & 72.1\end{array}$

$\begin{array}{lll}2.5 & 74.2 & 68.5\end{array}$

$\begin{array}{lll}0.0 & 44.6 & 50.0\end{array}$

$\begin{array}{lll}0.0 & 47.4 & 50.0\end{array}$

$\begin{array}{lll}0.0 & 44.6 & 50.0\end{array}$

$\begin{array}{lll}0.0 & 39.1 & 50.0\end{array}$

$\begin{array}{lll}2.5 & 74.0 & 68.5\end{array}$

$\begin{array}{lll}8.5 & 65.0 & 112.7\end{array}$

$\begin{array}{lll}3.0 & 89.4 & 72.1\end{array}$

$\begin{array}{lll}3.0 & 86.5 & 72.1\end{array}$

$\begin{array}{lll}4.0 & 56.8 & 79.5\end{array}$

$\begin{array}{lll}1.5 & 73.8 & 61.1\end{array}$

$\begin{array}{lll}1.0 & 79.7 & 57.4\end{array}$

$\begin{array}{lll}0.5 & 62.2 & 53.7\end{array}$

$\begin{array}{lll}0.0 & 46.5 & 50.0\end{array}$

$\begin{array}{rrr}18.0 & 232.6 & 182.9\end{array}$

2-hexadecanoyloxy-1,3-bis-(9-cis-octadecenoyloxy)

$\begin{array}{lll}3.5 & 82.9 & 75.8\end{array}$

propane

2-hexanone

2-hydroxybenzoic acid

2-hydroxymethyl-2-methyl-1,3-propanediol

2-iodobenzoic acid

2-iodonaphthalene

2-isopropoxyphenyl N-methylcarbamate

2-methoxy-4H-1,3,2-benzodioxaphosphorin 2-sulfide

2-methyl-1,3-butadiene

2-methyl-1-butene

2-methyl-1-phenyl-2-(N-piperidinyl)-1-propanone

2-methyl-1-propanol

2-methyl-2-(methylsulfonyl)propanal oxime

404.3

$\begin{array}{lll}2.5 & 68.4 & 68.5\end{array}$

$\begin{array}{lll}0.0 & 57.0 & 50.0\end{array}$

$\begin{array}{lll}2.0 & 76.9 & 64.8\end{array}$

$\begin{array}{lll}0.0 & 49.1 & 50.0\end{array}$

$\begin{array}{lll}0.0 & 49.0 & 50.0\end{array}$

$\begin{array}{lll}4.0 & 63.2 & 79.5\end{array}$

$\begin{array}{lll}0.5 & 51.6 \quad 53.7\end{array}$

$\begin{array}{lll}0.0 & 38.6 & 50.0\end{array}$

$\begin{array}{lll}0.5 & 58.3 \quad 53.7\end{array}$

$\begin{array}{lll}0.0 & 54.0 & 79.5\end{array}$

$1.0 \quad 36.9 \quad 57.4$

2-methyl-2(methylthio)propionaldehyde

O-methylcarbamoyloxime

1.0

$71.0 \quad 57.4$

2-methyl-2-butanethiol

2-methyl-2-butanol

2-methyl-2-butene

2-methyl-2-diphenylmethyl-1,3-cyclopentanedione

2-methyl-2-nitro-1,3-propandiol

2-methyl-2-nitro-1-propanol

2-methyl-2-nitropropyl-4,4,4-trinitrobutyrate

2-methyl-4,6-dinitrophenol

$\begin{array}{lll}4.5 & 60.7 & 83.2 \\ 2.0 & 52.5 & 64.8 \\ 2.0 & 31.1 & 64.8 \\ 0.0 & 54.4 & 50.0 \\ 2.5 & 87.0 & 68.5 \\ 2.0 & 82.1 & 64.8 \\ 1.0 & 65.8 & 57.4 \\ 5.5 & 85.7 & 90.6 \\ 0.0 & 54.0 & 50.0\end{array}$


2-methylbutane

2-methylcyclothiapentane

2-methyldecane

2-methylfuran

2-methylheptane

2-methylhexane

2-methylnaphthalene

2-methylnonane

2-methylpentane

2-methylpiperidine

2-methylpyridine

2-methyltetrazole

2-methylthiazole

2-methylthiophene

2-naphthoic acid

2-naphthyl benzoate

2-naphthylamine

2-n-butyl-5-(4-bromobiphenyl-4-yl)thiophene

2-nitro-1-(4-nitrophenoxy)-4-(trifluoromethyl)benzene

2-nitro-5-methylphenol

2-nitroaniline

2-nonadecanone

2-n-propyl-5-(4-bromophenyl)thiophene

2-octanone

2-pentadecanone

2-pentanol

2-pentanone

2-phenylbenzimidazole

2-piperadone

2-propanethiol

2-propanol

2-pyrrolidone

2-sec-butyl-4,6-dinitrophenol

2-sec-butyl-4,6-dinitrophenyl 3-methylcrotonate

2-tetradecanone

2-undecanone

3-(1-methylethyl)-(1H)-2,1,3-benzothiadiazin-4(3H)-one

2,2-dioxide

3-(3,4-dichlorophenyl)-1,1-dimethylurea

3-(4-chlorophenyl)-1,1-dimethylurea

3-(5-(1,1-dimethylethyl)-1,3,4-thiadiazol-2-yl)-

4-hydroxy-1-methyl-2-imidazolidinone

3(n-decylamino)-1,2-propanediol

3(n-decyloxy)-1,2-propanediol

3(n-decylthio)-1,2-propanediol

3(n-dodecylamino)-1,2-propanediol

3(n-dodecyloxy)-1,2-propanediol

3(n-dodecylthio)-1,2-propanediol

3(n-heptylamino)-1,2-propanediol

3(n-heptyloxy)-1,2-propanediol

3(n-heptylthio)-1,2-propanediol

3(n-hexyloxy)-1,2-propanediol

3(n-hexylthio)-1,2-propanediol

$\begin{array}{rrr}1.0 & 45.2 & 57.4 \\ 2.0 & 51.5 & 64.8 \\ 7.0 & 111.7 & 101.7 \\ 0.0 & 47.0 & 50.0 \\ 4.0 & 72.6 & 79.5 \\ 3.0 & 59.3 & 72.1 \\ 0.0 & 58.9 & 50.0 \\ 6.0 & 88.0 & 94.3 \\ 2.0 & 52.4 & 64.8 \\ 3.0 & 69.0 & 72.1 \\ 0.0 & 47.1 & 50.0 \\ 0.0 & 43.3 & 50.0 \\ 0.0 & 48.9 & 50.0 \\ 0.0 & 45.6 & 50.0 \\ 0.0 & 51.2 & 50.0 \\ 1.0 & 68.8 & 57.4 \\ 0.0 & 60.4 & 50.0 \\ 3.5 & 42.7 & 75.8 \\ 4.0 & 50.6 & 79.5 \\ 0.0 & 68.7 & 50.0 \\ 0.0 & 46.8 & 50.0 \\ 16.0 & 209.3 & 168.1 \\ 2.0 & 43.6 & 64.8 \\ 4.5 & 96.6 & 83.2 \\ 12.0 & 174.2 & 138.6 \\ 2.0 & 42.4 & 64.8 \\ 1.5 & 56.3 & 61.1 \\ 0.0 & 38.8 & 44.3 \\ 2.5 & 47.0 & 68.5 \\ 0.0 & 40.5 & 50.0 \\ 0.0 & 29.2 & 50.0 \\ 1.5 & 46.6 & 61.1 \\ 1.5 & 69.5 & 61.1 \\ 5.0 & 55.3 & 86.9 \\ 11.0 & 160.2 & 131.2 \\ 7.5 & 99.1 & 105.4 \\ & & \\ 0.5 & 52.8 & 53.7 \\ 2.0 & 78.9 & 64.8 \\ 2.0 & 65.8 & 64.8 \\ & & \\ 1.5 & 62.3 & 61.1 \\ 13.0 & 158.1 & 146.0 \\ 13.0 & 125.1 & 146.0 \\ 13.0 & 114.7 & 146.0 \\ 15.0 & 176.5 & 160.7 \\ 15.0 & 159.1 & 160.7 \\ 15.0 & 122.9 & 160.7 \\ 9.0 & 88.6 & 116.4 \\ 9.0 & 104.1 & 116.4 \\ 9.0 & 100.1 & 116.4 \\ 8.0 & 37.4 & 109.1 \\ 8.0 & 166.8 & 109.1\end{array}$


3(n-nonylamino)-1,2-propanediol

3-(n-nonyloxy)-1,2-propanediol

3(n-octylamino)-1,2-propanediol

3(n-octyloxy)-1,2-propanediol

3(n-octylthio)-1,2-propanediol

3(n-tetradecylamino)-1,2-propanediol

3(n-tetradecyloxy)-1,2-propanediol

3(n-tetradecylthio)-1,2-propanediol

3(n-tridecylamino)-1,2-propanediol

3(n-tridecyloxy)-1,2-propanediol

3(n-tridecylthio)-1,2-propanediol

3(n-undecylamino)-1,2-propanediol

3(n-undecyloxy)-1,2-propanediol

3(n-undecylthio)-1,2-propanediol

3-(p-tolyl-4-sulfonyl)-1-butyl urea

3,3',4'4'-tetraaminodiphenyl ether

3,3',5,5'-tetra-tert-butyldiphenylmethane-4,4'-diol

3,3'-bis-(1-cyclohexylethyl)-5,5'-dimethyldiphenyl

methane-2,2'-diol

3,3-bis-(chloromethyl)oxacyclobutane

3,3-diethylpentane

3,3-dimethyl-1-(methylthio)-2-butanone

O-methylcarbamoyloxime

3,3-dimethyl-1-butene

3,3-dimethyl-2-butanone

3,3-dimethylpentane

3,3-dimethylpentanedioic anhydride

3,3'-di-tert-butyl-5,5'-dimethyl-2,2-dihydroxydiphenyl

methane

3,4-benzophenanthrene

3,4-benzopyrene

3,4-dichloro-2-methoxybenzoic acid

3,4-dichlorophenol

3',4'-dichloropropionanilide

3,4-diethyl-3,4-bis(4-tert-butylphenyl)hexane

3,4-dimethylisoxazol 5-sulphanylamide

3,4-dimethylphenol

3,4-dimethylphenyl methylcarbamate

3,4-dimethylpyridine

3,4-dinitrophenol

3,4-dinitrotoluene

3,5,6-trichloro-2-pyridinol

3,5,6-trichloro-2-pyridinyloxyacetic acid

3,5-dibromo-4-hydroxybenzonitrile

3,5-dichlorobenzoic acid

3,5-dichloro-N-(1,1-dimethyl-2-propynyl)benzamide

3,5-dichlorophenol

3,5-diisopropylphenol

3,5-dimethyl-4-(dimethylamino)phenyl methylcarbamate

3,5-dimethylbenzoic acid

3,5-dimethylphenol

3,5-dimethylpyridine

3,5-dinitrobenzoic acid

$\begin{array}{rrr}11.0 & 155.0 & 131.2 \\ 11.0 & 99.3 & 131.2 \\ 10.0 & 134.3 & 123.8 \\ 10.0 & 112.8 & 123.8 \\ 10.0 & 129.9 & 123.8 \\ 17.0 & 182.2 & 175.5 \\ 17.0 & 187.4 & 175.5 \\ 17.0 & 133.6 & 175.5 \\ 16.0 & 193.6 & 168.1 \\ 16.0 & 158.5 & 168.1 \\ 16.0 & 106.7 & 168.1 \\ 14.0 & 166.9 & 153.3 \\ 14.0 & 138.3 & 153.3 \\ 14.0 & 99.1 & 153.3 \\ 5.0 & 63.3 & 86.9 \\ 1.0 & 62.8 & 57.4 \\ 5.0 & 96.0 & 86.9 \\ & & \\ 10.0 & 73.1 & 123.8 \\ 2.0 & 58.0 & 64.8 \\ 4.0 & 48.2 & 79.5\end{array}$

$\begin{array}{lll}4.5 & 60.1 & 83.2\end{array}$

$\begin{array}{lll}0.0 & 41.7 & 50.0\end{array}$

$\begin{array}{lll}0.0 & 51.2 & 50.0\end{array}$

$\begin{array}{lll}2.0 & 51.2 & 64.8\end{array}$

$\begin{array}{lll}0.0 & 45.4 & 44.3\end{array}$

$\begin{array}{lll}3.0 & 72.7 & 72.1\end{array}$

$\begin{array}{lll}0.0 & 48.8 & 50.0\end{array}$

$\begin{array}{lll}0.0 & 59.9 & 50.0\end{array}$

$\begin{array}{lll}1.0 & 85.6 & 57.4\end{array}$

$\begin{array}{lll}0.0 & 61.4 & 50.0\end{array}$

$\begin{array}{lll}2.0 & 50.2 & 64.8\end{array}$

$\begin{array}{lll}8.0 & 74.3 & 109.1\end{array}$

$\begin{array}{lll}1.0 & 62.5 & 57.4\end{array}$

$\begin{array}{lll}0.0 & 54.3 & 50.0\end{array}$

$\begin{array}{lll}2.0 & 71.2 & 64.8\end{array}$

$\begin{array}{lll}0.0 & 56.0 & 50.0\end{array}$

$\begin{array}{lll}0.0 & 62.3 & 50.0\end{array}$

$\begin{array}{lll}0.0 & 57.1 & 50.0\end{array}$

$\begin{array}{lll}0.0 & 57.6 & 50.0\end{array}$

$\begin{array}{lll}2.0 & 73.6 & 64.8\end{array}$

$\begin{array}{lll}0.0 & 69.0 & 44.3\end{array}$

$\begin{array}{lll}0.0 & 50.0 & 44.3\end{array}$

$\begin{array}{lll}2.0 & 66.9 & 64.8\end{array}$

$\begin{array}{lll}0.0 & 60.1 & 44.3\end{array}$

$\begin{array}{lll}0.0 & 37.2 & 61.1\end{array}$

$\begin{array}{lll}3.0 & 50.8 & 72.1\end{array}$

$\begin{array}{lll}0.0 & 51.0 & 44.3\end{array}$

$\begin{array}{lll}0.0 & 53.4 & 44.3\end{array}$

$\begin{array}{lll}0.0 & 49.1 & 44.3\end{array}$

$\begin{array}{lll}0.0 & 47.5 & 50.0\end{array}$ 
3,6-dichloro-2-methoxybenzoic acid

3,6-dichloro-5-hydroxy-2-methoxybenzoic acid

3-amino-2,5-dichlorobenzoic acid

3-aminoacetophenone

3-aminobenzoic acid

3-bromopentane

3-chloro-1,1,1,3,3-pentafluoropropane

3 -chlorobenzoic acid

3-chlorophenol

3-deoxy-3-fluoro-D-glucopyranose

3-deoxy-D-glucopyranose

3-diphenylmethyl-2,4-pentanedione

3-ethyl-3-diphenylmethyl-2,4-pentanedione

3-ethylheptane

3-ethylpentane

3-fluorotoluene

3-heptanone

3-hexanone

3-hydroxybenzoic acid

3-iodobenzoic acid

3-methyl-1,2-butadiene

3-methyl-1-butanethiol

3-methyl-1-butene

3-methyl-1-phenyl-1H-pyrazol-5-yl dimethylcarbamate

3-methyl-2-butanethiol

3-methyl-3-diphenylmethyl-2,4-pentanedione

3-methylcyclothiapentane

3-methylheptane

3-methylpentane

3-methylpyridine

3-methylthiophene

3-nitroaniline

3-nitrophthalic anhydride

3-nitrotoluene

3-pentanol

3-pentanone

3-propyl-3-diphenylmethyl-2,4-pentanedione

4-(1,1-dimethylethyl)-n-(1-methylpropyl)-

2,6-dinitrobenzeneamine

4-(2,4,5-trichlorophenoxy)butanoic acid

4-(2,4-dichlorophenoxy)butyric acid

4-(2-chlorophenylhydrazone)-3-methyl-5-isoxazolone

4-(4-chloro-2-methylphenoxy)butanoic acid

4-(4-nitrophenylazo)aniline

4-(6-hexenyloxy)-3',4'difluorodiphenyldiacetylene

4-(cis-3-hexenyloxy)-3',4'difluorodiphenyldiacetylene

4-(cis-4-hexenyloxy)-3',4'difluorodiphenyldiacetylene

4-(dipropylamino)-N,N-dimethyl-

3,5-dinitrobenzenesulfonamide

4-(N,N-dipropylamino)-3,5-dinitrobenzenesulphonamide

4,4'-di-(2-methoxyethoxy)biphenyl

4,4'dichlorodiphenylsulphone

4,4'-didecanoyloxydiphenyldiacetylene

$\begin{array}{rrr}1.0 & 59.2 & 57.4 \\ 1.0 & 70.7 & 57.4 \\ 0.0 & 78.7 & 50.0 \\ 0.0 & 78.1 & 50.0 \\ 0.0 & 48.2 & 50.0 \\ 2.0 & 50.2 & 64.8 \\ 0.0 & 63.3 & 50.0 \\ 0.0 & 55.8 & 50.0 \\ 0.0 & 48.8 & 50.0 \\ 3.0 & 48.4 & 72.1 \\ 3.0 & 84.2 & 72.1 \\ 2.0 & 69.8 & 64.8 \\ 3.0 & 89.4 & 72.1 \\ 5.0 & 101.4 & 86.9 \\ 3.0 & 61.8 & 72.1 \\ 0.0 & 45.1 & 50.0 \\ 3.5 & 74.3 & 75.8 \\ 2.5 & 66.6 & 68.5 \\ 0.0 & 55.1 & 50.0 \\ 0.0 & 62.3 & 50.0 \\ 0.0 & 49.8 & 44.3 \\ 2.0 & 53.1 & 64.8 \\ 0.5 & 51.2 & 53.7 \\ 2.0 & 66.0 & 64.8 \\ 1.0 & 52.5 & 57.4 \\ 2.0 & 71.3 & 64.8 \\ 2.0 & 54.0 & 64.8 \\ 4.0 & 76.5 & 79.5 \\ 2.0 & 48.1 & 64.8 \\ 0.0 & 55.6 & 50.0 \\ 0.0 & 51.6 & 50.0 \\ 0.0 & 61.2 & 50.0 \\ 0.0 & 42.2 & 50.0 \\ 0.0 & 51.9 & 50.0 \\ 2.0 & 44.5 & 64.8 \\ 1.5 & 50.0 & 61.1 \\ 4.0 & 77.6 & 79.5 \\ & & \\ 6.0 & 61.5 & 94.3 \\ 4.0 & 78.3 & 79.5 \\ 4.0 & 98.2 & 79.5 \\ 1.5 & 63.7 & 61.1 \\ 4.0 & 85.8 & 79.5 \\ 1.0 & 65.3 & 57.4 \\ 5.0 & 101.2 & 86.9 \\ 4.0 & 84.9 & 79.5 \\ 4.0 & 96.9 & 79.5 \\ & & \\ 6.0 & 78.7 & 94.3 \\ 4.5 & 92.8 & 83.2 \\ 8.0 & 97.5 & 109.1 \\ 0.0 & 257.8 & 50.0 \\ & & 182.9\end{array}$


4,4'-didodecanoyloxydiphenyldiacetylene

$\begin{array}{rrrr}1 & 22.0 & 244.0 & 212.4 \\ 1 & 1.0 & 69.5 & 57.4 \\ 1 & 1.0 & 24.6 & 57.4 \\ 1 & 20.0 & 165.3 & 197.6 \\ 1 & 1.0 & 73.0 & 57.4 \\ 1 & 8.0 & 86.1 & 109.1 \\ 1 & 1.0 & 16.6 & 57.4 \\ 1 & 1.5 & 56.4 & 61.1 \\ & & & \\ 1 & 0.5 & 45.1 & 53.7 \\ 1 & 0.0 & 100.2 & 50.0 \\ 2 & 0.0 & 45.3 & 44.3 \\ & & & \\ 1 & 2.0 & 49.2 & 64.8 \\ 2 & 0.0 & 46.7 & 44.3 \\ 1 & 0.0 & 65.0 & 50.0 \\ 1 & 0.0 & 64.4 & 50.0 \\ 1 & 0.0 & 64.4 & 50.0 \\ 2 & 0.0 & 75.3 & 44.3 \\ 2 & 0.0 & 62.9 & 44.3 \\ 2 & 0.0 & 38.2 & 44.3 \\ 1 & 4.0 & 78.2 & 79.5 \\ 2 & 0.0 & 44.5 & 44.3 \\ 1 & 2.0 & 84.4 & 64.8 \\ 1 & 1.0 & 65.6 & 57.4 \\ 1 & 1.0 & 64.5 & 57.4 \\ 1 & 2.0 & 84.7 & 64.8 \\ 1 & 3.0 & 77.0 & 72.1 \\ 1 & 2.0 & 62.2 & 64.8 \\ 1 & 4.0 & 15.5 & 79.5 \\ 1 & 3.0 & 63.9 & 72.1 \\ 1 & 1.0 & 36.4 & 57.4 \\ 1 & 2.0 & 57.1 & 64.8 \\ 2 & 0.0 & 43.2 & 44.3 \\ 1 & 3.5 & 67.3 & 75.8 \\ 1 & 4.5 & 55.8 & 83.2 \\ 2 & 0.0 & 69.6 & 44.3 \\ 2 & 0.0 & 77.6 & 44.3 \\ 2 & 0.0 & 63.3 & 44.3 \\ 1 & 1.0 & 67.0 & 57.4 \\ 1 & 2.0 & 71.8 & 64.8 \\ 2 & 0.0 & 64.8 & 44.3 \\ 1 & 1.0 & 62.0 & 57.4 \\ 1 & 0.5 & 55.7 & 53.7 \\ 1 & 2.0 & 60.9 & 64.8 \\ 1 & 4.0 & 76.5 & 79.5 \\ 1 & 3.0 & 75.6 & 72.1 \\ 1 & 0.0 & 64.2 & 50.0 \\ 1 & 3.0 & 52.0 & 72.1 \\ 1 & 0.5 & 57.5 & 53.7 \\ 1 & 0.0 & 63.3 & 50.0 \\ 1 & 2.0 & 43.2 & 64.8 \\ 1 & 4.0 & 71.2 & 79.5\end{array}$

4,4'dihydroxydiphenyl-2,2-propane

4,4'-dinitrodiphenyl ether

4,4'-diundecanoyloxydiphenyldiacetylene

4,6-dichloro-N-(2-chlorophenyl)-1,3,5-triazin-2-amine

4 [p-[bis(2-chloroethyl)amino]benzene]butanoic acid

4'4'-diaminodiphenyl ether

4-acetoxybenzoic acid

4-amino-6-(1,1-dimethylethyl)-3-(methylthio)

1,2,4-triazin-5(4H)-one

4-aminoacetophenone

4-aminobenzoic acid

4-amino-N-(6-methoxy-3-pyridazinyl)

benzenesulfonamide

4-aminopyridine

4-benzoyl-1-naphthol

4-bromo-2,5-dichloroaniline

4-bromo-2,5-dichlorophenol

4-chloroazobenzene

4-chlorobenzoic acid

4-chlorobiphenyl

4-chlorobut-2-ynyl 3-chlorophenylcarbamate

4-chlorophenol

4-chlorophenoxyacetic acid

4-chlorophenyl 4-chlorobenzenesulfonate

4-chlorophenylbenzenesulfonate

4-ethoxy-4'fluorodiphenyldiacetylene

4-ethoxy-4'-trifluoromethyldiphenyldiacetylene

4-ethoxybenzoic acid

4-ethoxyisonitrosoacetanilide

4-ethoxyphenylacetic acid

4-ethylbenzoic acid

4-ethynyl-1-[(4-ethynylphenyl)methoxy]benzene

4-fluorotoluene

4-heptanone

4-hexylresorcinol

4-hydroxy-3,5-diiodobenzonitrile

4-hydroxyazobenzene

4-hydroxybenzoic acid

4-hydroxyphenylacetic acid

4-hydroxyphenylpropionic acid

4-iodobenzoic acid

4-methoxybenzoic acid

4-methoxyphenol

4-methoxyphenylacetic acid

4-methoxyphenylbutyric acid

4-methoxyphenylpropionic acid

4-methyl-7-aminocoumarin

4-methyl-7-diethylaminocoumarin

4-methyl-7-dimethylaminocoumarin

4-methyl-7-hydroxycoumarin

4-methylcyclohex-1-ene

4-methylheptane 


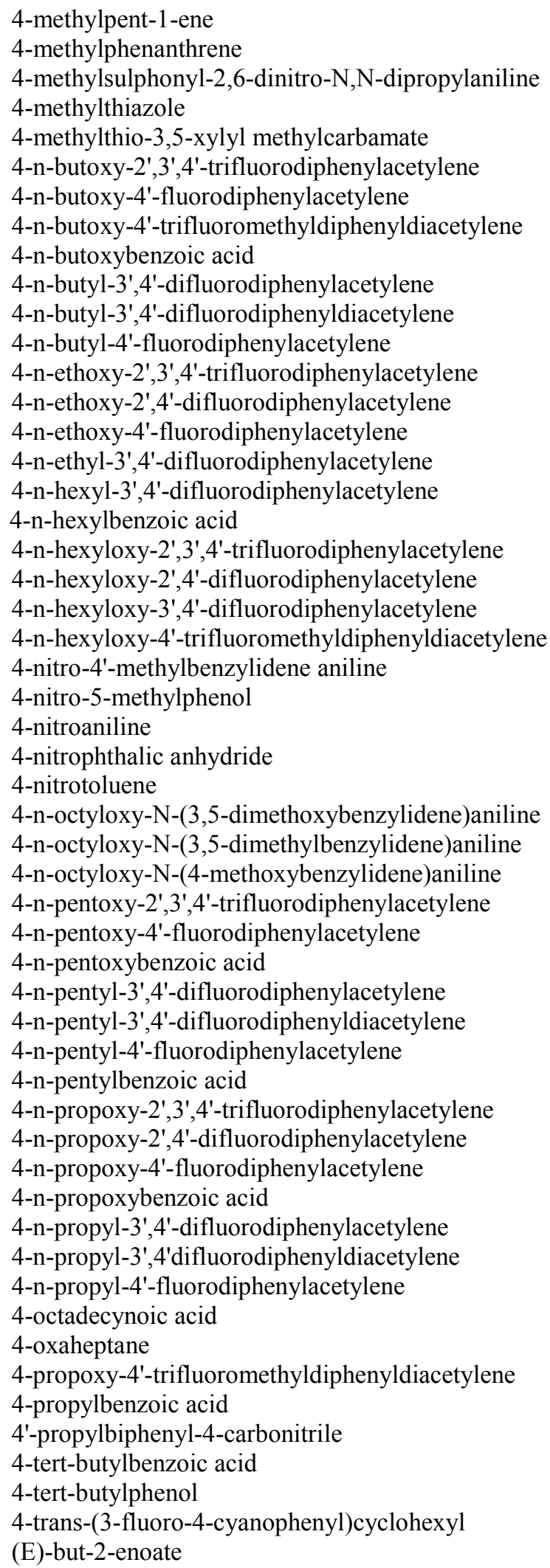

\begin{tabular}{|c|c|c|}
\hline 1.5 & 41.5 & 61.1 \\
\hline 0.0 & 43.4 & 50.0 \\
\hline 5.0 & 66.1 & 86.9 \\
\hline 0.0 & 38.8 & 50.0 \\
\hline 3.0 & 77.1 & 72.1 \\
\hline 4.0 & 104.5 & 79.5 \\
\hline 4.0 & 73.3 & 79.5 \\
\hline 5.0 & 61.2 & 86.9 \\
\hline 4.0 & 51.5 & 79.5 \\
\hline 3.0 & 78.2 & 72.1 \\
\hline 3.0 & 71.4 & 72.1 \\
\hline 3.0 & 56.1 & 72.1 \\
\hline 2.0 & 90.2 & 64.8 \\
\hline 2.0 & 78.6 & 64.8 \\
\hline 2.0 & 64.3 & 64.8 \\
\hline 1.0 & 55.1 & 57.4 \\
\hline 5.0 & 77.2 & 86.9 \\
\hline 5.0 & 53.2 & 86.9 \\
\hline 6.0 & 95.7 & 94.3 \\
\hline 6.0 & 106.3 & 94.3 \\
\hline 6.0 & 102.3 & 94.3 \\
\hline 7.0 & 86.1 & 101.7 \\
\hline 1.0 & 67.9 & 57.4 \\
\hline 0.0 & 68.3 & 50.0 \\
\hline 0.0 & 50.2 & 44.3 \\
\hline 0.0 & 44.2 & 50.0 \\
\hline 0.0 & 51.8 & 44.3 \\
\hline 10.0 & 111.6 & 123.8 \\
\hline 8.0 & 116.2 & 109.1 \\
\hline 9.0 & 112.1 & 116.4 \\
\hline 5.0 & 104.8 & 86.9 \\
\hline 5.0 & 82.2 & 86.9 \\
\hline 5.0 & 59.7 & 86.9 \\
\hline 4.0 & 68.4 & 79.5 \\
\hline 4.0 & 86.9 & 79.5 \\
\hline 4.0 & 75.9 & 79.5 \\
\hline 4.0 & 41.5 & 79.5 \\
\hline 3.0 & 79.7 & 72.1 \\
\hline 3.0 & 77.1 & 72.1 \\
\hline 3.0 & 76.0 & 72.1 \\
\hline 3.0 & 65.9 & 72.1 \\
\hline 2.0 & 65.0 & 64.8 \\
\hline 2.0 & 64.1 & 64.8 \\
\hline 2.0 & 74.4 & 64.8 \\
\hline 14.0 & 166.5 & 153.3 \\
\hline 4.0 & 68.0 & 79.5 \\
\hline 4.0 & 59.5 & 79.5 \\
\hline 2.0 & 66.5 & 64.8 \\
\hline 2.0 & 67.0 & 64.8 \\
\hline 0.0 & 40.7 & 50.0 \\
\hline 0.0 & 38.9 & 50.0 \\
\hline 5.0 & 53.7 & 86.9 \\
\hline
\end{tabular}


4-trans-(4-bromophenyl)cyclohexyl (E)-2-butenoate 4-trans-(4-chlorophenyl)cyclohexyl (E)-2-butenoate 4-trans-(4-fluorophenyl)cyclohexyl (E)-2-butenoate 4-trans-(4-fluorophenylethyl)cyclohexyl (E)-butenoate 4-trans-(trifluoromethoxyphenyl)cyclohexyl

(E)-but-2-enoate

4-trans-cyanocyclohexyl (E) 2-butenoate

5'-(trifluoromethanesulphonamide)acet-2',4-xylidide

5,5-bis(3,3-dimethylbutyl)2,2,8,8-tetramethylnonane

5,5-dimethylperhydro-1,3-oxazine-2-one

5,6,7,8-tetrahydroquinoline

5,6-dibutyl-5,6-bis(4-tert-butylphenyl)decane

5-[2-chloro-4-(trifluoromethyl)phenoxy]-

2-nitrobenzoic acid

5-amino-4-chloro-2-phenyl-3(2H)-pyridazinone

5-bromo-6-methyl-3-(1-methylpropyl)-2,4(1H,3H)pyrimidinedione

5-butyl-2-ethylamino-6-methylpyrimidin-4-ol

5-chloro-3-(1,1-dimethylethyl)-6-methyl-2,4(1H,3H)pyrimidinedione

5-isopropyl-m-tolyl methylcarbamate

5-methyl N-(methylcarbamoyloxy)thioacetimidate

5-methyl-1,2,4-triazolo[3,4-b]benzothiazole

5-methylnonane

5-methyltetrazole

5-methylthiazole

5-nonanone

5-octadecynoic acid

6-(4-biphenyl)-1-hexene

6,8,9-trimethyladenine

6,9-dimethyl-8-butyladenine

6-deoxy-6-fluoro-D-glucopyranose

6-deoxy-D-glucopyranose

6-methyl-1,3-dithiolo[4,5-b]quinoxalin-2-one

6-octadecynoic acid

7,8-benzoquinoline

7-octadecynoic acid

8-(4-biphenyl)-1-octene

8-[4-(4'-n-butylbiphenyl)]-1-octene

8-octadecynoic acid

9-fluorenone

9-heptadecanone

9H-pyrido[3,4-b]indole

9-methylfluorene

9-octadecynoic acid

a-(trifluoromethoxy)-a,a-difluoromethyl acetate

a,a'-dibromo-m-xylene

a,a'-dibromo-o-xylene

a,a'-dichloro-m-xylene

a,a'-dichloro-o-xylene

a,a'-dichloro-p-xylene

a-alanyl-a-alanine (DL)

acenaphthene

$\begin{array}{rrrr}1 & 5.0 & 73.2 & 86.9 \\ 1 & 5.0 & 78.2 & 86.9 \\ 1 & 5.0 & 70.9 & 86.9 \\ 1 & 7.0 & 74.6 & 101.7 \\ & & & \\ 1 & 6.0 & 63.5 & 94.3 \\ 1 & 4.5 & 66.6 & 83.2 \\ 1 & 2.0 & 82.4 & 64.8 \\ 1 & 13.0 & 102.7 & 146.0 \\ 1 & 2.5 & 64.8 & 68.5 \\ 1 & 1.0 & 40.8 & 57.4 \\ 1 & 16.0 & 111.7 & 168.1 \\ & & & \\ 1 & 3.0 & 86.3 & 72.1 \\ 1 & 1.5 & 55.8 & 61.1 \\ & & & \\ 1 & 1.5 & 51.4 & 61.1 \\ 1 & 4.5 & 47.0 & 83.2 \\ & & & \\ 1 & 0.0 & 27.9 & 50.0 \\ 1 & 3.0 & 63.8 & 72.1 \\ 1 & 3.5 & 61.6 & 75.8 \\ 1 & 0.0 & 52.3 & 50.0 \\ 1 & 6.0 & 89.2 & 94.3 \\ 2 & 0.0 & 38.3 & 44.3 \\ 1 & 0.0 & 32.9 & 50.0 \\ 1 & 5.5 & 117.5 & 90.6 \\ 1 & 14.0 & 167.4 & 153.3 \\ 1 & 4.0 & 55.0 & 79.5 \\ 1 & 0.5 & 52.7 & 53.7 \\ 1 & 3.5 & 88.0 & 75.8 \\ 1 & 3.0 & 66.0 & 72.1 \\ 1 & 3.0 & 55.5 & 72.1 \\ 1 & 0.0 & 67.5 & 50.0 \\ 1 & 14.0 & 169.5 & 153.3 \\ 1 & 0.0 & 43.5 & 50.0 \\ 1 & 14.0 & 166.5 & 153.3 \\ 1 & 6.0 & 72.0 & 94.3 \\ 1 & 9.0 & 39.3 & 116.4 \\ 1 & 14.0 & 172.8 & 153.3 \\ 2 & 0.0 & 50.8 & 44.3 \\ 1 & 14.0 & 205.9 & 153.3 \\ 1 & 0.0 & 54.1 & 50.0 \\ 1 & 0.0 & 51.1 & 50.0 \\ 1 & 14.0 & 172.0 & 153.3 \\ 1 & 2.5 & 50.8 & 68.5 \\ 1 & 1.5 & 67.6 & 61.1 \\ 1 & 1.5 & 72.7 & 61.1 \\ 1 & 1.5 & 63.5 & 61.1 \\ 1 & 1.5 & 64.8 & 61.1 \\ 1 & 1.5 & 64.2 & 61.1 \\ 1 & 3.0 & 68.7 & 72.1 \\ 2 & 0.0 & 58.5 & 44.3 \\ & & & \end{array}$




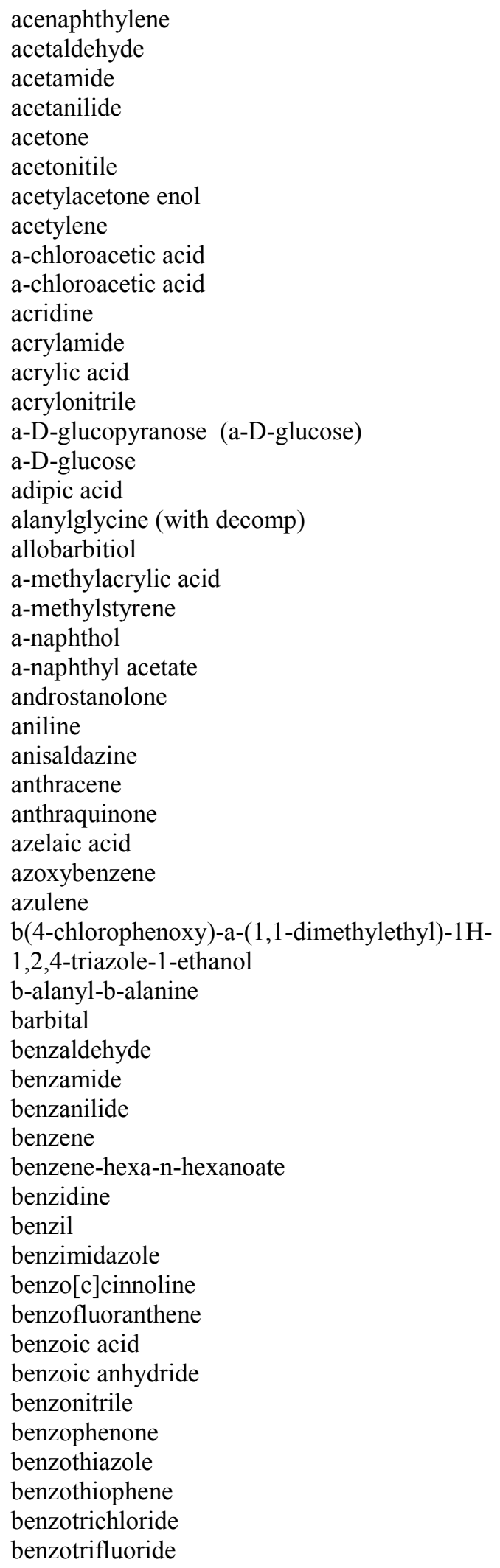

\begin{tabular}{|c|c|c|c|}
\hline 2 & 0.0 & 42.4 & 44.3 \\
\hline 1 & 0.0 & 22.5 & 50.0 \\
\hline 1 & 0.0 & 44.2 & 50.0 \\
\hline 1 & 1.0 & 55.9 & 57.4 \\
\hline 2 & 0.0 & 32.4 & 44.3 \\
\hline 1 & 0.0 & 39.5 & 50.0 \\
\hline 1 & 0.0 & 56.9 & 50.0 \\
\hline 2 & 0.0 & 37.3 & 44.3 \\
\hline 1 & 0.5 & 42.2 & 53.7 \\
\hline 1 & 0.5 & 48.8 & 53.7 \\
\hline 2 & 0.0 & 48.5 & 44.3 \\
\hline 1 & 0.0 & 42.8 & 50.0 \\
\hline 2 & 0.0 & 39.1 & 44.3 \\
\hline 1 & 0.0 & 39.1 & 50.0 \\
\hline 1 & 3.0 & 81.0 & 72.1 \\
\hline 1 & 3.0 & 75.9 & 72.1 \\
\hline 1 & 4.0 & 81.7 & 79.5 \\
\hline 1 & 3.0 & 111.4 & 72.1 \\
\hline 1 & 2.5 & 73.0 & 68.5 \\
\hline 1 & 0.0 & 28.0 & 50.0 \\
\hline 1 & 0.0 & 47.5 & 50.0 \\
\hline 1 & 0.0 & 62.4 & 50.0 \\
\hline 1 & 1.0 & 63.3 & 57.4 \\
\hline 1 & 0.0 & 59.6 & 50.0 \\
\hline 2 & 0.0 & 39.5 & 44.3 \\
\hline 1 & 2.0 & 67.3 & 64.8 \\
\hline 4 & 0.0 & 60.1 & 38.5 \\
\hline 4 & 0.0 & 58.4 & 38.5 \\
\hline 1 & 7.0 & 86.0 & 101.7 \\
\hline 1 & 1.5 & 58.0 & 61.1 \\
\hline 2 & 0.0 & 46.9 & 44.3 \\
\hline 1 & 4.0 & 64.8 & 79.5 \\
\hline 1 & 3.0 & 121.4 & 72.1 \\
\hline 1 & 1.5 & 54.0 & 61.1 \\
\hline 2 & 0.0 & 43.2 & 44.3 \\
\hline 1 & 0.0 & 46.0 & 50.0 \\
\hline 1 & 1.0 & 67.8 & 57.4 \\
\hline 2 & 0.0 & 35.4 & 29.4 \\
\hline 1 & 30.0 & 281.7 & 271.5 \\
\hline 4 & 0.0 & 47.7 & 38.5 \\
\hline 1 & 0.0 & 64.5 & 50.0 \\
\hline 2 & 0.0 & 43.4 & 44.3 \\
\hline 2 & 0.0 & 48.4 & 44.3 \\
\hline 1 & 0.0 & 43.6 & 50.0 \\
\hline 2 & 0.0 & 45.5 & 44.3 \\
\hline 1 & 1.0 & 54.8 & 57.4 \\
\hline 2 & 0.0 & 42.2 & 44.3 \\
\hline 2 & 0.0 & 56.7 & 44.3 \\
\hline 1 & 0.0 & 46.4 & 50.0 \\
\hline 1 & 0.0 & 38.9 & 50.0 \\
\hline 1 & 0.0 & 59.1 & 50.0 \\
\hline 1 & 0.0 & 56.4 & 50.0 \\
\hline
\end{tabular}


benzoxazole

benzyl alcohol

benzyl benzoate

benzylbromide

benzyliodide

benzylmethylsulfone

biphenyl

bis(2-cyanoethyl)-N-nitroamine

bis-(4-aminophenyl)methane

bis(4-chlorophenyl)acetic acid

bis-[3,5-di-tert-butyl-4-hydroxybenzyl]sulfide

bis-hydroxyethylpiperazine

bromobenzene

bromocyclohexane

bromomethane

bromotrichloromethane

butanal

butane

butanoic acid

butyl 4-aminobenzoate

butyl 9-hydroxy-9H-fluorene-9-carboxylate

butyl acrylate

butyl alcohol

butyl butanoate

butyl ethyl sulfide

butyl octadecanoate

caffeine

carbazole

carbon tetrabromide

carbon tetrachloride

carbon tetrafluoride

chlorobenzene

chlorocyclohexane

chlorocyclopentane

chlorodifluoromethane

chloroethane

chloroethyl methacrylate

chloropentafluorobenzene

chlorotrifluoroethylene

cholesterol*

chroman

chromone

chrysene

cinnamic acid

cinnamic anhydride

cinnamyl alcohol

cis,cis-1,3,5-tri-tert-butylcyclohexane

cis,trans-1,3,5-tri-tert-butylcyclohexane

cis-1,2-cyclohexanediol

cis-1,2-dimethylcyclohexane

cis-1,2-dimethylcyclopentane

cis-1,3-dimethylcyclohexane

cis-1,4-dimethylcyclohexane

$\begin{array}{rrr}0.0 & 55.5 & 50.0 \\ 0.5 & 34.1 & 53.7 \\ 2.0 & 69.7 & 64.8 \\ 0.5 & 48.6 & 53.7 \\ 0.5 & 44.1 & 53.7 \\ 0.5 & 63.7 & 53.7 \\ 0.0 & 54.6 & 38.5 \\ 4.0 & 137.6 & 79.5 \\ 1.0 & 25.4 & 57.4 \\ 1.0 & 71.9 & 57.4 \\ 7.0 & 103.3 & 101.7 \\ 6.0 & 64.0 & 94.3 \\ 0.0 & 44.1 & 44.3 \\ 3.0 & 49.7 & 72.1 \\ 0.0 & 33.3 & 50.0 \\ 0.0 & 28.7 & 40.9 \\ 1.5 & 62.7 & 61.1 \\ 1.0 & 53.6 & 57.4 \\ 1.5 & 41.8 & 61.1 \\ 4.0 & 61.8 & 79.5 \\ 4.0 & 74.3 & 79.5 \\ 4.0 & 82.6 & 79.5 \\ 2.0 & 50.5 & 64.8 \\ 5.5 & 82.2 & 90.6 \\ 4.0 & 69.6 & 79.5 \\ 20.0 & 132.8 & 197.6 \\ 0.0 & 47.6 & 50.0 \\ 0.0 & 52.2 & 44.3 \\ 0.0 & 29.5 & 29.4 \\ 0.0 & 31.3 & 29.4 \\ 0.0 & 27.0 & 29.4 \\ 0.0 & 41.9 & 44.3 \\ 3.0 & 45.7 & 72.1 \\ 2.0 & 48.6 & 64.8 \\ 0.0 & 36.2 & 50.0 \\ 0.0 & 33.0 & 50.0 \\ 3.0 & 72.3 & 72.1 \\ 0.0 & 55.5 & 44.3 \\ 0.0 & 48.3 & 50.0 \\ 4.0 & 73.4 & 79.5 \\ 1.0 & 60.3 & 57.4 \\ 0.0 & 52.4 & 50.0 \\ 0.0 & 55.3 & 44.3 \\ 1.0 & 55.7 & 57.4 \\ 3.0 & 102.0 & 72.1 \\ 1.5 & 51.0 & 61.1 \\ 2.0 & 68.1 & 64.8 \\ 2.0 & 53.2 & 64.8 \\ 3.0 & 64.1 & 72.1 \\ 3.0 & 55.2 & 72.1 \\ 2.0 & 54.6 & 64.8 \\ 3.0 & 54.8 & 72.1 \\ 3.0 & 50.0 & 72.1\end{array}$




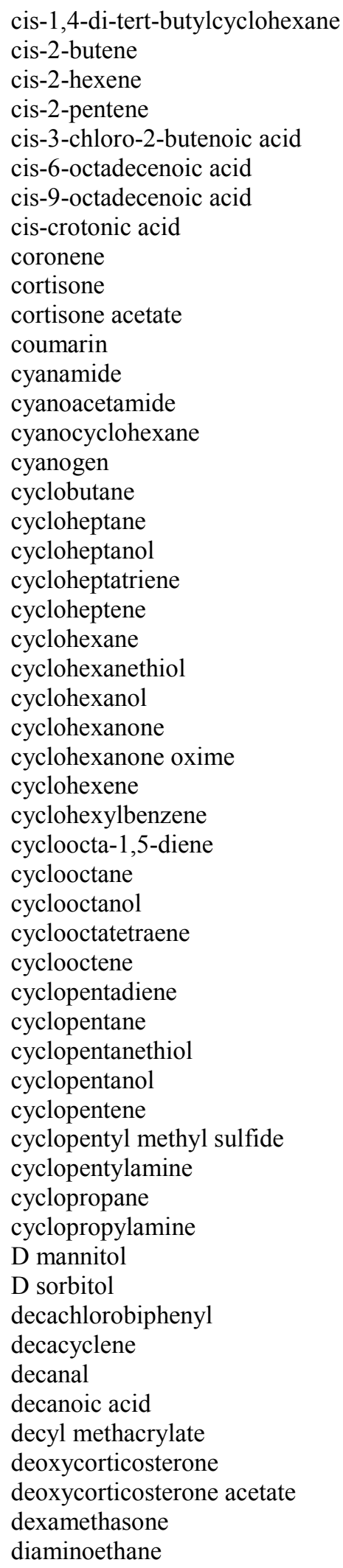

\begin{tabular}{|c|c|c|}
\hline 4.0 & 30.0 & 79.5 \\
\hline 0.0 & 54.4 & 44.3 \\
\hline 2.0 & 67.3 & 64.8 \\
\hline 1.0 & 58.4 & 57.4 \\
\hline 0.5 & 41.4 & 53.7 \\
\hline 14.0 & 156.4 & 153.3 \\
\hline 14.0 & 138.2 & 153.3 \\
\hline 0.5 & 36.5 & 53.7 \\
\hline 0.0 & 27.0 & 29.4 \\
\hline 0.5 & 74.5 & 53.7 \\
\hline 2.0 & 75.5 & 64.8 \\
\hline 0.0 & 55.9 & 50.0 \\
\hline 0.0 & 27.6 & 30.9 \\
\hline 0.5 & 59.1 & 53.7 \\
\hline 3.0 & 47.3 & 72.1 \\
\hline 0.0 & 33.1 & 44.3 \\
\hline 0.0 & 45.1 & 44.3 \\
\hline 4.0 & 47.6 & 79.5 \\
\hline 4.0 & 28.5 & 79.5 \\
\hline 1.0 & 17.7 & 57.4 \\
\hline 3.0 & 42.1 & 72.1 \\
\hline 3.0 & 45.8 & 72.1 \\
\hline 3.0 & 52.7 & 72.1 \\
\hline 3.0 & 39.3 & 72.1 \\
\hline 2.5 & 44.6 & 68.5 \\
\hline 2.0 & 35.4 & 64.8 \\
\hline 2.0 & 49.9 & 64.8 \\
\hline 2.5 & 54.5 & 68.5 \\
\hline 3.0 & 48.2 & 72.1 \\
\hline 5.0 & 48.9 & 86.9 \\
\hline 5.0 & 15.1 & 86.9 \\
\hline 0.0 & 41.9 & 38.5 \\
\hline 4.0 & 58.5 & 79.5 \\
\hline 0.0 & 45.4 & 44.3 \\
\hline 2.0 & 46.0 & 64.8 \\
\hline 2.0 & 50.4 & 64.8 \\
\hline 2.0 & 24.3 & 64.8 \\
\hline 1.0 & 29.8 & 57.4 \\
\hline 2.5 & 59.4 & 68.5 \\
\hline 2.0 & 46.2 & 64.8 \\
\hline 0.0 & 37.4 & 35.1 \\
\hline 0.0 & 55.4 & 44.3 \\
\hline 5.0 & 127.8 & 86.9 \\
\hline 5.0 & 82.4 & 86.9 \\
\hline 0.0 & 68.1 & 38.5 \\
\hline 0.0 & 38.1 & 50.0 \\
\hline 7.5 & 114.1 & 105.4 \\
\hline 7.5 & 91.4 & 105.4 \\
\hline 9.0 & 121.9 & 116.4 \\
\hline 0.5 & 67.6 & 53.7 \\
\hline 2.0 & 69.0 & 64.8 \\
\hline 0.5 & 78.0 & 53.7 \\
\hline 1.0 & 81.2 & 57.4 \\
\hline
\end{tabular}




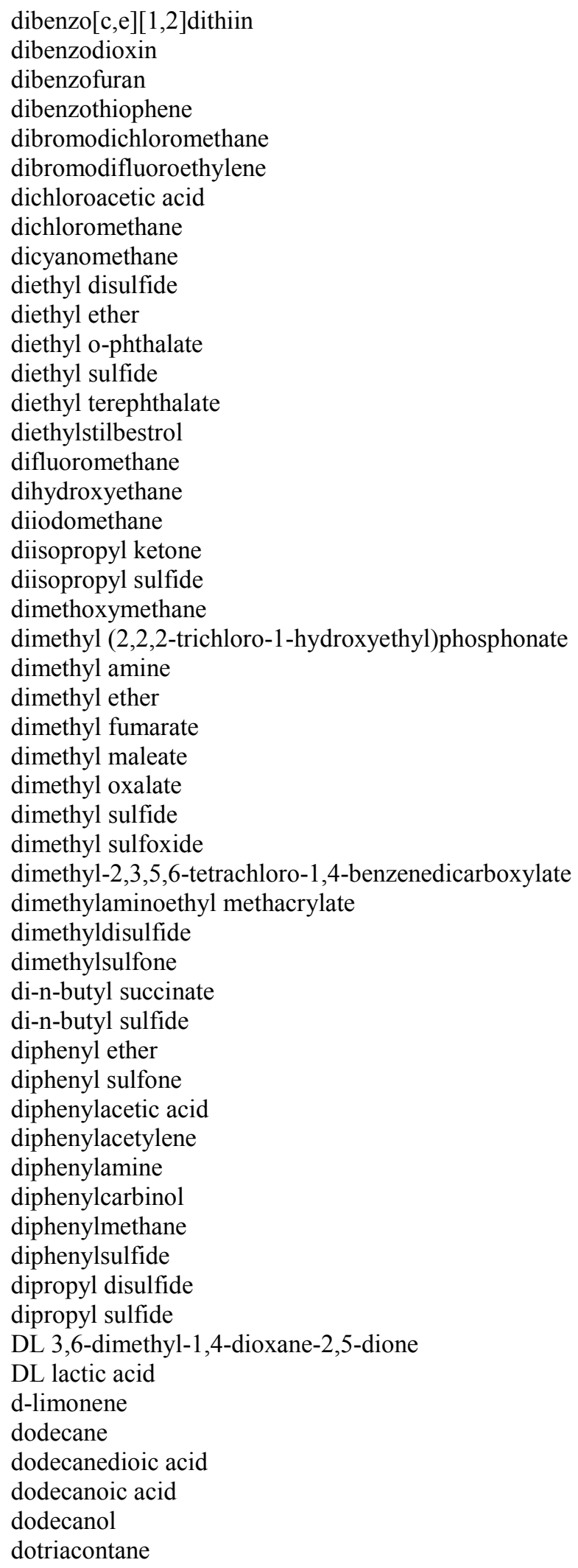

\begin{tabular}{|c|c|c|c|}
\hline 2 & 0.0 & 50.0 & 44.3 \\
\hline 4 & 0.0 & 58.6 & 38.5 \\
\hline 2 & 0.0 & 52.3 & 44.3 \\
\hline 2 & 0.0 & 58.2 & 44.3 \\
\hline & 0.0 & 28.8 & 44.3 \\
\hline$?$ & 0.0 & 43.2 & 44.3 \\
\hline 1 & 0.0 & 43.1 & 50.0 \\
\hline & 0.0 & 34.6 & 50.0 \\
\hline 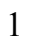 & 0.0 & 35.4 & 50.0 \\
\hline & 3.0 & 54.8 & 72.1 \\
\hline 1 & 2.0 & 45.8 & 64.8 \\
\hline 1 & 4.5 & 66.7 & 83.2 \\
\hline 1 & 2.0 & 70.4 & 64.8 \\
\hline 1 & 4.5 & 77.8 & 83.2 \\
\hline 1 & 3.0 & 71.6 & 72.1 \\
\hline 1 & 0.0 & 32.0 & 50.0 \\
\hline 1 & 1.0 & 38.2 & 57.4 \\
\hline 1 & 0.0 & 43.2 & 50.0 \\
\hline 1 & 1.5 & 54.7 & 61.1 \\
\hline 1 & 2.0 & 53.4 & 64.8 \\
\hline 1 & 2.0 & 49.6 & 64.8 \\
\hline 1 & 2.0 & 65.1 & 64.8 \\
\hline 1 & 0.0 & 29.7 & 50.0 \\
\hline 1 & 0.0 & 37.5 & 50.0 \\
\hline 1 & 3.0 & 93.7 & 72.1 \\
\hline 1 & 3.0 & 57.6 & 72.1 \\
\hline 1 & 2.0 & 64.3 & 64.8 \\
\hline 1 & 0.0 & 45.6 & 50.0 \\
\hline 2 & 0.0 & 49.3 & 44.3 \\
\hline 1 & 2.5 & 70.0 & 68.5 \\
\hline 1 & 4.0 & 70.9 & 79.5 \\
\hline 1 & 1.0 & 48.8 & 57.4 \\
\hline 2 & 0.0 & 47.9 & 44.3 \\
\hline 1 & 10.0 & 119.7 & 123.8 \\
\hline 1 & 6.0 & 93.9 & 94.3 \\
\hline 1 & 1.0 & 57.4 & 57.4 \\
\hline 1 & 0.0 & 54.7 & 50.0 \\
\hline 1 & 1.0 & 74.3 & 57.4 \\
\hline 4 & 0.0 & 61.4 & 38.5 \\
\hline 1 & 1.0 & 54.8 & 57.4 \\
\hline 1 & 1.0 & 67.9 & 57.4 \\
\hline 1 & 1.0 & 62.3 & 57.4 \\
\hline 1 & 1.0 & 54.2 & 57.4 \\
\hline 1 & 5.0 & 73.6 & 86.9 \\
\hline 1 & 4.0 & 71.2 & 79.5 \\
\hline 2 & 0.0 & 62.1 & 44.3 \\
\hline 1 & 0.5 & 39.1 & 53.7 \\
\hline 1 & 1.5 & 57.1 & 61.1 \\
\hline 1 & 9.0 & 139.7 & 116.4 \\
\hline 1 & 10.0 & 125.6 & 123.8 \\
\hline 1 & 9.5 & 115.7 & 120.1 \\
\hline 1 & 10.0 & 133.8 & 123.8 \\
\hline 1 & 30.0 & 345.1 & 271.5 \\
\hline
\end{tabular}




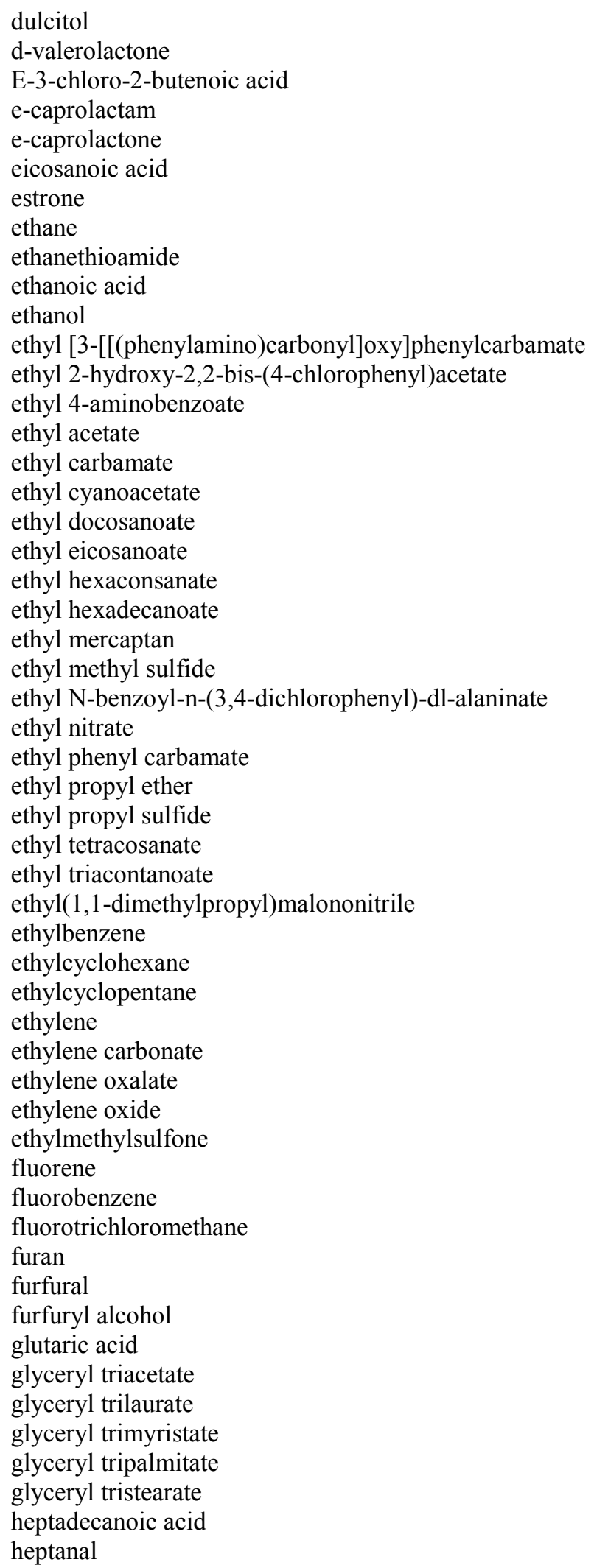

\begin{tabular}{|c|c|c|}
\hline 5.0 & 141.4 & 86.9 \\
\hline 2.5 & 42.9 & 68.5 \\
\hline 0.5 & 41.4 & 53.7 \\
\hline 3.5 & 46.9 & 75.8 \\
\hline 3.5 & 50.8 & 75.8 \\
\hline 18.0 & 198.7 & 182.9 \\
\hline 0.0 & 90.1 & 50.0 \\
\hline 0.0 & 31.2 & 25.2 \\
\hline 0.0 & 47.6 & 50.0 \\
\hline 0.0 & 39.2 & 44.3 \\
\hline 0.0 & 57.4 & 50.0 \\
\hline 5.0 & 83.1 & 86.9 \\
\hline 3.0 & 75.6 & 72.1 \\
\hline 2.0 & 64.9 & 64.8 \\
\hline 1.5 & 55.4 & 61.1 \\
\hline 1.5 & 47.3 & 61.1 \\
\hline 2.5 & 47.7 & 68.5 \\
\hline 22.0 & 90.4 & 212.4 \\
\hline 20.0 & 69.3 & 197.6 \\
\hline 26.0 & 124.8 & 241.9 \\
\hline 16.0 & 50.9 & 168.1 \\
\hline 0.0 & 25.4 & 50.0 \\
\hline 1.0 & 58.4 & 57.4 \\
\hline 4.0 & 79.2 & 79.5 \\
\hline 0.0 & 47.8 & 50.0 \\
\hline 2.0 & 49.9 & 64.8 \\
\hline 3.0 & 57.6 & 72.1 \\
\hline 3.0 & 67.8 & 72.1 \\
\hline 28.0 & 105.3 & 256.7 \\
\hline 30.0 & 154.0 & 271.5 \\
\hline 3.0 & 62.6 & 72.1 \\
\hline 0.5 & 51.4 & 53.7 \\
\hline 3.5 & 51.6 & 75.8 \\
\hline 2.5 & 50.9 & 68.5 \\
\hline 0.0 & 32.2 & 38.5 \\
\hline 1.5 & 43.0 & 61.1 \\
\hline 2.0 & 32.3 & 64.8 \\
\hline 0.0 & 32.2 & 44.3 \\
\hline 0.0 & 36.7 & 50.0 \\
\hline 0.0 & 50.5 & 44.3 \\
\hline 0.0 & 49.0 & 44.3 \\
\hline 0.0 & 42.4 & 40.9 \\
\hline 0.0 & 33.9 & 44.3 \\
\hline 0.0 & 61.1 & 50.0 \\
\hline 0.5 & 50.7 & 53.7 \\
\hline 3.0 & 63.0 & 72.1 \\
\hline 6.5 & 93.7 & 98.0 \\
\hline 36.0 & 386.6 & 315.8 \\
\hline 42.0 & 460.9 & 360.0 \\
\hline 48.0 & 529.3 & 404.3 \\
\hline 54.0 & 588.0 & 448.6 \\
\hline 15.0 & 176.2 & 160.7 \\
\hline 4.5 & 99.8 & 83.2 \\
\hline
\end{tabular}


heptane

heptanoic acid

hexachlorobenzene

hexachlorocyclopropane

hexachloroethane

hexacontane

hexadecane

hexadecanoic acid

hexafluoroacetone

hexafluorobenzene

hexafluoroethane

hexamethylbenzene

hexanal

hexanamide

hexatriacontane

hexyl ethanoate

hexyl N-phenylcarbamate

hydrazobenzene (1,2-diphenylhydrazine)

hydrocinnamic acid

hydrocortisone

hydrocortisone-21-acetate

imidazole

indene

iodobenzene

iodomethane

isobutane

isobutene

isobutyl alcohol

isobutyl mercaptan

isochroman

isonicotinic acid

isopropyl 4,4'-dibromobenzilate

isopropyl ether

isopropyl methyl ketone

isopropyl methyl sulfide

isopropyl nitrate

isopropyl phenylcarbamate

isopropyl-3-chlorophenylcarbamate

isopropylbenzene

isoquinoline

L-carvone

levulinic acid

L-iditol

linoelaidic acid

maleic anhydride

malonamide

m-aminophenol

m-bromophenol

methacrylamide

methanethiol

methanol

methoxybenzene

methyl 2-(2,4,5-trichlorophenoxy)acetate

$\begin{array}{rrr}4.0 & 76.9 & 79.5 \\ 4.5 & 67.2 & 83.2 \\ 0.0 & 47.2 & 29.4 \\ 0.0 & 49.5 & 35.1 \\ 0.0 & 53.2 & 35.1 \\ 58.0 & 500.4 & 478.2 \\ 14.0 & 176.8 & 153.3 \\ 14.0 & 163.3 & 153.3\end{array}$

$\begin{array}{lll}0.0 & 56.7 & 50.0\end{array}$

$\begin{array}{lll}0.0 & 41.6 & 29.4\end{array}$

$\begin{array}{lll}0.0 & 51.4 & 25.2\end{array}$

$\begin{array}{lll}0.0 & 51.0 & 29.4\end{array}$

$\begin{array}{lll}3.5 & 63.3 & 75.8 \\ 3.5 & 67.1\end{array}$

$\begin{array}{lll}3.5 & 67.1 & 75.8\end{array}$

$34.0 \quad 371.1 \quad 301.0$

$\begin{array}{lll}5.5 & 93.5 & 90.6\end{array}$

$\begin{array}{lll}6.0 & 99.9 & 94.3\end{array}$

$\begin{array}{lll}2.0 & 43.3 & 64.8\end{array}$

$\begin{array}{lll}2.0 & 55.0 & 64.8\end{array}$

$\begin{array}{lll}0.5 & 73.7 & 53.7\end{array}$

$\begin{array}{lll}2.0 & 74.5 & 64.8\end{array}$

$\begin{array}{lll}0.0 & 35.4 & 50.0\end{array}$

$\begin{array}{lll}0.0 & 37.5 & 50.0\end{array}$

$\begin{array}{lll}0.0 & 40.3 & 44.3\end{array}$

$\begin{array}{lll}0.0 & 44.1 & 40.9\end{array}$

$\begin{array}{lll}0.0 & 40.1 & 40.9\end{array}$

$\begin{array}{lll}0.0 & 44.7 & 44.3\end{array}$

$\begin{array}{lll}1.0 & 36.9 & 57.4\end{array}$

$\begin{array}{lll}1.0 & 38.8 & 57.4\end{array}$

$\begin{array}{lll}1.0 & 60.4 & 57.4\end{array}$

$\begin{array}{lll}0.0 & 54.2 & 44.3\end{array}$

$\begin{array}{lll}3.0 & 70.5 & 72.1\end{array}$

$\begin{array}{lll}2.0 & 64.2 & 64.8\end{array}$

$\begin{array}{lll}0.5 & 51.9 & 53.7\end{array}$

$\begin{array}{lll}1.0 & 54.5 & 57.4\end{array}$

$\begin{array}{lll}0.0 & 52.9 & 50.0\end{array}$

$\begin{array}{lll}3.0 & 53.9 & 72.1\end{array}$

$\begin{array}{lll}3.0 & 56.5 & 72.1\end{array}$

$\begin{array}{lll}0.5 & 41.3 & 53.7\end{array}$

$\begin{array}{lll}0.0 & 45.2 & 50.0\end{array}$

$\begin{array}{lll}0.0 & 46.6 & 50.0\end{array}$

$\begin{array}{lll}2.0 & 30.1 & 64.8\end{array}$

$\begin{array}{lrr}5.0 & 87.6 & 86.9\end{array}$

$12.0 \quad 157.4 \quad 138.6$

$\begin{array}{lll}0.0 & 37.6 & 44.3\end{array}$

$\begin{array}{lll}1.0 & 85.1 & 57.4\end{array}$

$\begin{array}{lll}0.0 & 57.6 & 50.0\end{array}$

$\begin{array}{lll}0.0 & 49.3 & 50.0\end{array}$

$\begin{array}{lll}0.0 & 39.0 & 50.0\end{array}$

$\begin{array}{lll}0.0 & 40.9 & 40.9\end{array}$

$\begin{array}{lll}0.0 & 21.5 & 30.9\end{array}$

$\begin{array}{lll}0.5 & 48.0 & 53.7\end{array}$

$\begin{array}{lll}3.0 & 84.2 & 72.1\end{array}$ 
methyl 2-(2,4,5-trichlorophenoxy)butyrate methyl 2-(2,4,5-trichlorophenoxy)propionate methyl 2-(4-(2,4-dichlorophenoxy)phenoxy)propionate methyl 2,4-dichlorophenoxyacetate methyl 3,6-dichloro-2-methoxybenzoate methyl 3-m-tolylcarbamoyloxyphenylcarbamate methyl 4-(2,4-dichlorophenoxy)butyrate methyl 4-amino-3,5,6-trichloro-2-picolinate methyl 4-aminobenzoate methyl 4-hydroxybenzoate methyl 5-(2,4-dichlorophenoxy)-2-nitrobenzoate methyl acetate methyl acrylate methyl a-D-mannopyranoside methyl benzoate methyl butyl sulfide methyl carbamate methyl chloride methyl docosanoate methyl isopropyl ether methyl methacrylate methyl myristate methyl n-butyl ether methyl n-decyl ether methyl nitrate methyl N-phenylcarbamate methyl octadecanoate methyl palmitate methyl perfluorobutanoate methyl p-N,N-dimethylaminobenzoate methyl propyl ether methyl propyl sulfide methyl tert-butyl ether methyl tert-butyl sulfide methyl tetrachloroterephthalic acid ester methyl-3,4-dichlorophenylcarbamate methylamine methylcyclobutane methylcyclohexane methylcyclopentane methylenecyclobutane methylhydrazine methylphenylsulfide methylphosphonyl chlorofluoride methylphosphonyl dichloride methylphosphonyl difluoride m-hydroxytoluene m-nitrobenzoic acid m-nitrophenol mono(2,2-dimethylhydrazide) butanedioic acid $\mathrm{m}$-phenylenediamine m-terphenyl m-toluic acid

$\begin{array}{rrr}4.0 & 91.2 & 79.5 \\ 3.0 & 88.6 & 72.1 \\ 4.0 & 86.1 & 79.5 \\ 3.0 & 79.6 & 72.1 \\ 2.0 & 60.7 & 64.8 \\ 4.0 & 93.5 & 79.5 \\ 5.0 & 105.4 & 86.9 \\ 1.0 & 67.9 & 57.4 \\ 1.0 & 58.6 & 57.4 \\ 1.0 & 61.0 & 57.4 \\ 3.0 & 73.4 & 72.1 \\ 0.5 & 42.8 & 53.7 \\ 1.0 & 49.3 & 57.4 \\ 4.0 & 98.2 & 79.5 \\ 1.0 & 53.3 & 57.4 \\ 3.0 & 70.9 & 72.1 \\ 0.5 & 50.8 & 53.7 \\ 0.0 & 36.8 & 30.9 \\ 21.0 & 89.9 & 205.0 \\ 1.0 & 46.0 & 57.4 \\ 1.0 & 54.4 & 57.4 \\ 13.0 & 172.2 & 146.0 \\ 3.0 & 68.9 & 72.1 \\ 9.0 & 130.2 & 116.4 \\ 0.0 & 43.3 & 50.0 \\ 2.0 & 44.8 & 64.8 \\ 17.0 & 61.9 & 175.5 \\ 15.0 & 221.8 & 160.7 \\ 2.5 & 61.5 & 68.5 \\ 2.0 & 70.1 & 64.8 \\ 2.0 & 57.2 & 64.8 \\ 2.0 & 61.9 & 64.8 \\ 0.0 & 46.2 & 50.0 \\ 0.0 & 44.1 & 50.0 \\ 1.5 & 38.0 & 61.1 \\ 2.0 & 60.8 & 64.8 \\ 0.0 & 34.1 & 30.9 \\ 1.0 & 41.6 & 57.4 \\ 3.0 & 45.6 & 72.1 \\ 2.0 & 53.0 & 64.8 \\ 0.5 & 42.3 & 53.7 \\ 0.0 & 47.2 & 50.0 \\ 0.5 & 57.9 & 53.7 \\ 0.0 & 47.3 & 50.0 \\ 0.0 & 59.1 & 44.3 \\ 0.0 & 50.3 & 44.3 \\ 0.0 & 37.5 & 50.0 \\ 0.0 & 46.7 & 50.0 \\ 0.0 & 51.7 & 50.0 \\ 4.0 & 85.7 & 79.5 \\ 0.0 & 45.9 & 44.3 \\ 0.0 & 62.8 & 44.3 \\ 0.0 & 41.2 & 50.0\end{array}$


m-toluidine

m-xylene

myo-inositol

N-(1-ethylpropyl)-2,6-dinitro-3,4-xylidine

$\begin{array}{lll}0.0 & 36.4 & 50.0\end{array}$

N-(2-chloroethyl)-2,6-dinitro-N-propyl-4-(trifluoromethyl)

benzeneamine

$\mathrm{N}(3), \mathrm{N}(3)$-diethyl-2,4-dinitro-6-(trifluoromethyl)-

1,3-benzenediamine

$\mathrm{N}$-(3,4-dichlorophenyl)-2-methyl-2-propenamide

N'-(3-chloro-4-methoxyphenyl)-N,N-dimethylurea

$\mathrm{N}$-(3-chloro-4-methylphenyl)-2-methylpentanamide

$\mathrm{N}$-(4-chlorophenyl)-2,2-dimethylpentanamide

N'-(4-chlorophenyl)-N-methoxy-N-methylurea

$\mathrm{N}$-(cyclopropylmethyl)-2,6-dinitro-n-propyl-4-

(trifluoromethyl)benzenamine

N,N'-(2-hydroxyethyl)-1,4-diaminoanthraquinone

N,N-(2-hydroxyethyl)-4-(4-nitrophenyl)azoaniline

$\mathrm{N}, \mathrm{N}$-(2-hydroxyethyl)-4-phenylazoaniline

N,N-diethyl-2-(1-naphthyloxy)propionamide

N,N-dimethyl-1,3-propanediamine

N,N-dimethyl-2,2-diphenylacetamide

N,N-dimethyl-2,2-diphenylbenzeneacetamide

N,N-dimethyl-2-methylcarbamoyloxyimino-2-

(methylthio)acetamide

N,N-dimethyl-4-phenylazoaniline

$\mathrm{N}, \mathrm{N}$-dimethylaniline

N,N-dimethylformamide

$\mathrm{N}, \mathrm{N}$-dimethylhydrazine

$\mathrm{N}, \mathrm{N}$-dimethylhydrazine

$\mathrm{N}, \mathrm{N}$-dimethyl-N,N'dinitro-1,2-ethanediamine

N,N'-dimethyl-N,N'dinitro-1,6-hexanediamine

N,N-dimethyl-N'-[3-(trifluoromethyl)-phenyl]urea

N,N-dimethyl-N'-[4-(1-methylethyl)phenyl]urea

N,N'-di-n-hexyladipamide

$\mathrm{N}, \mathrm{N}$ '-dinitro-diaminomethane

$\mathrm{N}, \mathrm{N}^{\prime}$-dinitroethanediamine

$\mathrm{N}, \mathrm{N}^{\prime}$-di-n-propyladipamide

$\mathrm{N}$-[(1,1,2,2-tetrachloroethyl)thio]-4-cyclohexene-

1,2-dicarboximide

N-[4-(methoxymethyl)-1-[2-(2-thienyl)ethyl]-

4-piperidinyl]-N-phenyl-propanamide

$\mathrm{N}-[4-m e t h y l-3-[[($ trifluoromethyl)sulfonyl]amino]phenyl]

acetamide

N-[5-(1,1-dimethylethyl)-1,3,4-thiadiazol-2-yl]-N,N'-

dimethylurea

$\mathrm{N}$-acetyl-D-leucine amide

$\mathrm{N}$-acetylglycine amide

$\mathrm{N}$-acetylglycyl-L-prolinamide

$\mathrm{N}$-acetyl-L-alanine amide

$\mathrm{N}$-acetyl-L-isoleucinamide

$\mathrm{N}$-acetyl-L-prolyl-glycinamide

\begin{tabular}{|c|c|c|c|}
\hline 1 & 0.0 & 36.4 & 50.0 \\
\hline 2 & 0.0 & 51.4 & 44.3 \\
\hline 1 & 3.0 & 96.4 & 72.1 \\
\hline 1 & 6.0 & 76.9 & 94.3 \\
\hline 1 & 4.5 & 72.5 & 83.2 \\
\hline 1 & 2.5 & 78.3 & 68.5 \\
\hline 1 & 1.5 & 81.0 & 61.1 \\
\hline 1 & 3.0 & 68.8 & 72.1 \\
\hline 1 & 4.0 & 46.3 & 79.5 \\
\hline 1 & 4.0 & 64.7 & 79.5 \\
\hline 1 & 3.0 & 63.8 & 72.1 \\
\hline 1 & 7.0 & 73.6 & 101.7 \\
\hline 1 & 6.0 & 62.0 & 94.3 \\
\hline 1 & 6.0 & 67.0 & 94.3 \\
\hline 1 & 5.0 & 73.6 & 86.9 \\
\hline 1 & 5.0 & 71.2 & 86.9 \\
\hline 1 & 3.0 & 63.7 & 72.1 \\
\hline 1 & 2.0 & 62.5 & 64.8 \\
\hline 1 & 2.0 & 63.3 & 64.8 \\
\hline 1 & 5.0 & 81.1 & 86.9 \\
\hline 1 & 1.0 & 59.3 & 57.4 \\
\hline 1 & 0.5 & 46.3 & 53.7 \\
\hline 1 & 0.5 & 42.0 & 53.7 \\
\hline 1 & 1.0 & 51.6 & 57.4 \\
\hline 1 & 0.0 & 46.6 & 50.0 \\
\hline 1 & 3.0 & 147.1 & 72.1 \\
\hline 1 & 7.0 & 186.3 & 101.7 \\
\hline 1 & 2.0 & 68.7 & 64.8 \\
\hline 1 & 3.0 & 78.7 & 72.1 \\
\hline 1 & 16.0 & 94.6 & 168.1 \\
\hline 1 & 2.0 & 96.6 & 64.8 \\
\hline 1 & 3.0 & 65.6 & 72.1 \\
\hline 1 & 10.0 & 79.9 & 123.8 \\
\hline 1 & 2.0 & 99.8 & 64.8 \\
\hline 1 & 6.0 & 64.4 & 94.3 \\
\hline 1 & 2.0 & 88.8 & 64.8 \\
\hline 1 & 2.0 & 67.7 & 64.8 \\
\hline 1 & 4.0 & 50.0 & 79.5 \\
\hline 1 & 2.0 & 62.7 & 64.8 \\
\hline 1 & 4.5 & 71.2 & 83.2 \\
\hline 1 & 2.0 & 50.3 & 64.8 \\
\hline 1 & 4.0 & 78.9 & 79.5 \\
\hline 1 & 4.5 & 74.2 & 83.2 \\
\hline
\end{tabular}


$\mathrm{N}$-acetyl-pyrazinamide

$\mathrm{N}$-acetylsarcosinamide

$\mathrm{N}$-allyl-N-phenylthiourea

naphthalene

naphthalene 1,8-disulfide

naphthalene 1,8-disulfide s-oxide

N-benzylaniline

N-butyl dodecanamide

n-butyl mercaptan

$\mathrm{N}$-butyl tetradecanamide

n-butylbenzene

n-butylcyclohexane

n-butylcyclopentane

N-butyl-N'-(3,4-dichlorophenyl)-N-methylurea

N-butyl-N-ethyl-2,6-dinitro-4-trifluoromethylaniline

N-butylurea

n-decane

$\mathrm{N}$-decyl hexadecanamide

n-decyl-a-cyanoacrylate

$\mathrm{N}$-decylundecanamide

$\mathrm{N}$-dimethylaminosuccinamic acid

n-docosane

n-dodecylcyclohexane

n-dononacontahectane

n-eicosane

neopentyl-4,4,4-trinitrobutyrate

n-ethyl-a-cyanoacrylate

$\mathrm{N}$-ethylurea

n-hectane

n-heneicosane

n-heptacosane

n-heptadecane

N-heptylmyristamide

n-hexacosane

n-hexane

N-hexyl decanamide

$\mathrm{N}$-hexyl hexadecanamide

$\mathrm{N}$-hexyl tetradecanamide

nicotinic acid

$\mathrm{N}$-isopropylcarbazole

$\mathrm{N}$-isopropylurea

nitrobenzene

nitroethane

nitromethane

N-laurylnonanamide

$\begin{array}{lll}1.5 & 64.4 & 61.1 \\ 2.0 & 66.4 & 64.8 \\ 3.5 & 73.6 & 75.8 \\ 0.0 & 54.0 & 38.5 \\ 0.0 & 32.9 & 44.3 \\ 0.0 & 55.3 & 50.0 \\ 2.0 & 54.8 & 64.8\end{array}$

$\begin{array}{lll}14.0 & 121.1 & 153.3\end{array}$

$\begin{array}{rrr}2.0 & 66.4 & 64.8\end{array}$

$\begin{array}{lll}16.0 & 133.9 & 168.1\end{array}$

$\begin{array}{lll}2.5 & 60.6 & 68.5\end{array}$

$\begin{array}{lll}5.5 & 71.3 & 90.6\end{array}$

$\begin{array}{lll}4.5 & 68.5 & 83.2\end{array}$

$\begin{array}{llr}5.0 & 72.7 & 86.9\end{array}$

$\begin{array}{lll}8.0 & 107.8 & 109.1\end{array}$

$\begin{array}{lll}3.5 & 64.4 & 75.8\end{array}$

$\begin{array}{lll}7.0 & 117.9 & 101.7\end{array}$

$\begin{array}{lll}24.0 & 196.6 & 227.2\end{array}$

$\begin{array}{lll}10.0 & 141.9 & 123.8\end{array}$

$\begin{array}{lll}19.0 & 123.6 & 190.3\end{array}$

$\begin{array}{lll}4.0 & 85.7 & 79.5\end{array}$

$\begin{array}{lll}20.0 & 245.0 & 197.6\end{array}$

$\begin{array}{lll}13.0 & 177.1 & 146.0\end{array}$

$190.0 \quad 1751.2 \quad 1452.6$

$\begin{array}{lll}18.0 & 219.6 & 182.9\end{array}$

$\begin{array}{lll}4.5 & 67.7 & 83.2\end{array}$

$\begin{array}{lll}2.0 & 52.9 & 64.8\end{array}$

$\begin{array}{lll}1.5 & 39.4 & 61.1\end{array}$

$\begin{array}{lll}98.0 & 1004.0 & 773.4\end{array}$

$19.0 \quad 202.7 \quad 190.3$

$25.0 \quad 265.7 \quad 234.6$

$\begin{array}{lll}15.0 & 174.7 & 160.7\end{array}$

$\begin{array}{lll}19.0 & 163.6 & 190.3\end{array}$

$\begin{array}{lll}24.0 & 289.3 & 227.2\end{array}$

$\begin{array}{lll}3.0 & 73.6 & 72.1\end{array}$

$\begin{array}{lll}14.0 & 119.6 & 153.3\end{array}$

$20.0 \quad 166.1 \quad 197.6$

$\begin{array}{lll}18.0 & 151.9 & 182.9\end{array}$

$\begin{array}{lll}0.0 & 53.9 & 50.0\end{array}$

$\begin{array}{lll}1.0 & 47.4 & 57.4\end{array}$

$1.5 \quad 52.0 \quad 61.1$

$\begin{array}{lll}0.0 & 43.5 & 44.3\end{array}$

$\begin{array}{lll}0.0 & 53.6 & 50.0\end{array}$

$\begin{array}{lll}0.0 & 39.6 & 44.3\end{array}$

$\mathrm{N}$-methyl O-methyl O-2-chloro-4-tert-butylphenyl

$\begin{array}{lll}19.0 & 196.7 \quad 190.3\end{array}$

phosphoramidate

$\mathrm{N}$-methyl-2,4,6,N-tetranitroaniline

$\mathrm{N}$-methyl-2-chlorophenylcarbamic acid ester

$\mathrm{N}$-methylacetamide

$\mathrm{N}$-methylcarbazole

$\begin{array}{lll}2.5 & 66.2 & 68.5 \\ 0.5 & 64.2 & 53.7 \\ 2.0 & 60.1 & 64.8 \\ 0.5 & 32.0 & 53.7 \\ 0.0 & 47.3 & 44.3\end{array}$


$\mathrm{N}$-methyldiphenylacetamide

$\begin{array}{rrr}2.0 & 68.7 & 64.8 \\ 3.0 & 113.5 & 72.1 \\ 0.0 & 36.1 & 44.3 \\ 0.5 & 37.2 & 53.7 \\ 19.0 & 164.4 & 190.3 \\ 2.0 & 105.3 & 64.8 \\ 0.0 & 115.2 & 50.0 \\ 27.0 & 286.1 & 249.3 \\ 26.0 & 300.3 & 241.9 \\ 5.0 & 95.8 & 86.9 \\ 7.0 & 127.9 & 101.7 \\ 17.0 & 201.4 & 175.5 \\ 17.0 & 195.9 & 175.5 \\ 6.5 & 116.6 & 98.0 \\ 6.0 & 99.0 & 94.3 \\ 6.5 & 92.1 & 98.0 \\ 9.0 & 98.8 & 116.4 \\ 10.0 & 85.8 & 123.8 \\ 15.0 & 142.9 & 160.7 \\ 48.0 & 504.2 & 404.3 \\ 23.0 & 258.2 & 219.8 \\ 13.0 & 156.0 & 146.0 \\ 33.0 & 368.0 & 293.6\end{array}$

$\mathrm{N}$-methyl-N-nitrobutanamine

$\mathrm{N}$-methylpyrrole

$\mathrm{N}$-methylurea

$\mathrm{N}$-myristylheptanamide

$\mathrm{N}$-nitro-bis(N,N-cyanomethyl) amine

$\mathrm{N}$-nitro-N-methylaminomethane

n-nonacosane

n-octacosane

n-octane

n-octylbenzene

nonadecane

nonadecanoic acid

nonanal

nonane

nonanoic acid

nonyl acrylate

nonyl phenylcarbamate

$\mathrm{N}$-palmitoyl-pyrazinamide

n-pentacontane

n-pentacosane

n-pentadecane

n-pentatriacontane

N-phenyl-N[1-(2-phenylethyl)-4-piperidinyl]propanamide

(fentanyl)

n-propylbenzene

$\begin{array}{lll}4.0 & 63.0 & 79.5\end{array}$

n-propylcyclohexane

$\begin{array}{lll}1.5 & 53.4 & 61.1\end{array}$

$\mathrm{N}$-propylstearamide

$\mathrm{N}$-propylurea

$\mathrm{N}$-salicylidene-m-aminobenzoic acid

$\mathrm{N}$-stearylpropanamide

N-tert-butylurea

n-tetracosane

n-tetratetracontane

n-tricosane

n-tridecane

n-undecane

$\mathrm{N}$-vinylpyrrolidone

O-(2,4-dichlorophenyl) O-methyl-(1-methylethyl)

phosphoramidothioate

O-(2-chloro-4-nitrophenyl) O,O-dimethyl

phosphorothioate

O-(4-bromo-2,5-dichlorophenyl) O,O-dimethyl

phosphorothioate

O-(4-bromo-2,5-dichlorophenyl) O-methyl

phenylphosphonothioate

$\begin{array}{lll}4.5 & 58.2 & 83.2\end{array}$

$\begin{array}{lll}19.0 & 187.4 & 190.3\end{array}$

$\begin{array}{lll}2.5 & 38.4 & 68.5\end{array}$

$\begin{array}{lll}0.0 & 71.4 & 50.0\end{array}$

$\begin{array}{lll}19.0 & 165.6 & 190.3\end{array}$

$\begin{array}{lll}0.5 & 73.9 & 53.7\end{array}$

$\begin{array}{lll}22.0 & 266.8 & 212.4\end{array}$

$\begin{array}{lll}42.0 & 403.1 & 360.0\end{array}$

$\begin{array}{lll}21.0 & 237.7 & 205.0\end{array}$

$\begin{array}{lll}11.0 & 136.3 & 131.2\end{array}$

$\begin{array}{rrrr}1 & 8.0 & 118.6 & 109.1 \\ 1 & 1.0 & 53.4 & 57.4\end{array}$

$\mathrm{O}, \mathrm{O}, \mathrm{O}^{\prime}, \mathrm{O}^{\prime}$-tetramethyl O,O'-thiodi-p-phenylene

bis(phosphorothioate)

O,O-diethyl O-4-nitrophenyl phosphorothioate

O,O-diethyl O-quinoxalin-2-yl phosphothioate

O,O-diethyl-O-(3,5,6-trichloro-2-pyridyl) phosphate

1

$\begin{array}{lll}3.5 & 91.2 & 75.8\end{array}$

$\begin{array}{llll}1 & 2.5 & 89.8 & 68.5\end{array}$

$\begin{array}{llll}1 & 2.5 & 95.8 & 68.5\end{array}$

$\begin{array}{llll}1 & 2.0 & 90.7 & 64.8\end{array}$

$\begin{array}{llll}1 & 7.0 & 108.9 & 101.7\end{array}$

$\begin{array}{llll}1 & 4.5 & 56.5 & 83.2\end{array}$

$\begin{array}{llll}1 & 4.5 & 83.5 & 83.2\end{array}$

$\begin{array}{llll}1 & 4.5 & 50.0 & 83.2\end{array}$ 
O,O-diethyl-O-(3,5,6-trichloro-2-pyridyl)

phosphorothioate

O,O-diisopropyl S-2-phenylsulfonylaminoethyl

phosphorodithioate

O,O-dimethyl O-(2,4,5-trichlorophenyl)

phosphorothioate

O,O-dimethyl O-(4-aminosulfonylphenyl)

phosphorothioate

O,O-dimethyl O-4-nitrophenyl phosphorothioate

O,O-dimethyl S-[2-(methylamino)-2-oxoethyl]

phosphorodithioate

O,O-dimethyl S-phthalimidomethyl phosphorodithioate

O,O-dimethyl-O-(3,5,6-trichloro-2-pyridyl)

phosphorothioate

O,S-dimethyl phosphoroamidothioate

O-6-ethoxycarbonyl-5-methylpyrazolo[1,5-a]

pyrimidin-2-yl O,O-diethyl phosphorothioate

o-aminophenol

octadecanamide

octadecane

octadecanoic acid

octadecanol

octafluoropropane

octafluorotetrahydrothiophene

octanal

octanoic acid

octyl methacrylate

O-ethyl O-(4-nitrophenyl)phenylphosphonothioate

o-hydroxyacetanilide

o-hydroxybiphenyl

o-hydroxytoluene

o-nitrobenzoic acid

o-nitrophenol

o-phenylenediamine

o-phenylenepyrene

o-terphenyl

o-toluic acid

o-toluidine

o-xylene

$\mathrm{p}, \mathrm{p}^{\prime}$-dichlorobenzophenone

p-a-cumylphenol

p-aminobenzene sulphonamide

p-aminophenol

p-benzoquinone

p-bromotoluene

p-chlorobenzyl p-chlorophenyl sulfide

p-chlorotoluene

p-dioxanone

pentabromophenol

pentachloroaniline

pentachlorobenzene

$\begin{array}{rrrr}1 & 4.5 & 77.9 & 83.2 \\ 1 & 8.0 & 98.6 & 109.1 \\ & & & \\ 1 & 2.5 & 60.5 & 68.5 \\ & & & \\ 1 & 2.5 & 76.1 & 68.5 \\ 1 & 2.5 & 65.1 & 68.5 \\ & & & \\ 1 & 4.5 & 63.8 & 83.2 \\ 1 & 3.5 & 78.6 & 75.8 \\ & & & \\ 1 & 2.5 & 81.3 & 68.5 \\ 1 & 1.0 & 42.1 & 57.4 \\ & & & \\ 1 & 7.0 & 84.2 & 101.7 \\ 1 & 0.0 & 76.0 & 50.0 \\ 1 & 16.0 & 158.8 & 168.1 \\ 1 & 16.0 & 204.6 & 168.1 \\ 1 & 16.0 & 178.7 & 168.1 \\ 1 & 17.0 & 209.7 & 175.5 \\ 1 & 0.0 & 39.6 & 50.0 \\ 2 & 0.0 & 82.4 & 44.3 \\ 1 & 5.5 & 89.7 & 90.6 \\ 1 & 5.5 & 73.8 & 90.6 \\ 1 & 7.5 & 104.6 & 105.4 \\ 1 & 4.0 & 81.3 & 79.5 \\ 1 & 1.0 & 58.3 & 57.4 \\ 1 & 0.0 & 48.1 & 50.0 \\ 1 & 0.0 & 52.0 & 50.0 \\ 1 & 0.0 & 66.8 & 50.0 \\ 1 & 0.0 & 54.8 & 50.0 \\ 2 & 0.0 & 61.8 & 44.3 \\ 1 & 0.0 & 49.4 & 50.0 \\ 1 & 0.5 & 52.2 & 53.7 \\ 1 & 0.0 & 53.5 & 50.0 \\ 1 & 0.0 & 32.4 & 50.0 \\ 2 & 0.0 & 54.9 & 44.3 \\ 2 & 0.0 & 71.7 & 44.3 \\ 1 & 1.0 & 62.6 & 57.4 \\ 1 & 0.0 & 58.4 & 50.0 \\ 2 & 0.0 & 56.2 & 44.3 \\ 4 & 0.0 & 47.6 & 38.5 \\ 2 & 0.0 & 50.1 & 44.3 \\ 1 & 2.0 & 93.7 & 64.8 \\ 2 & 0.0 & 48.3 & 44.3 \\ 1 & 2.5 & 53.5 & 68.5 \\ 2 & 0.0 & 63.7 & 44.3 \\ 2 & 0.0 & 37.0 & 44.3 \\ 2 & 0.0 & 57.6 & 44.3\end{array}$




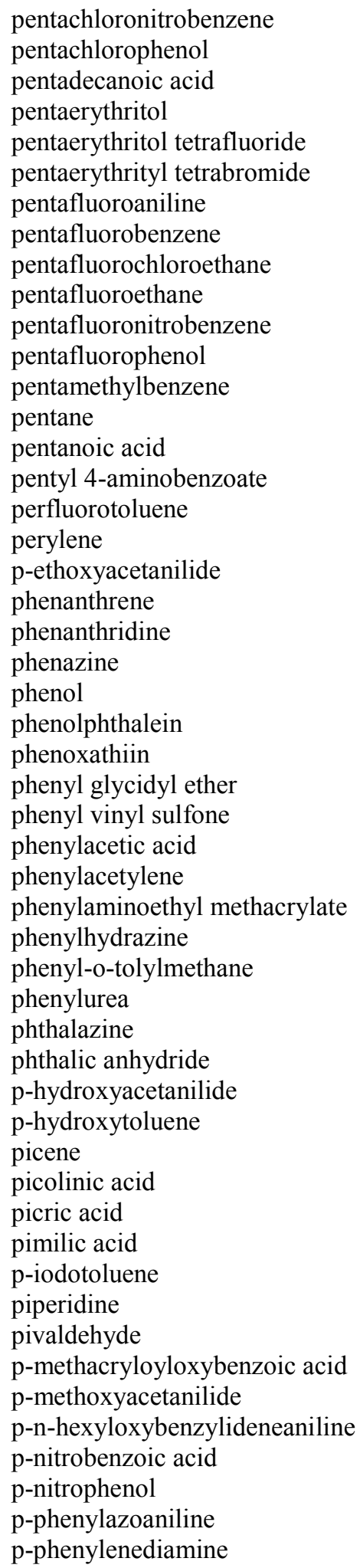

\begin{tabular}{|c|c|c|}
\hline 0.0 & 44.0 & 50.0 \\
\hline 0.0 & 37.1 & 44.3 \\
\hline 13.0 & 153.0 & 146.0 \\
\hline 3.0 & 108.8 & 72.1 \\
\hline 4.0 & 67.1 & 79.5 \\
\hline 4.0 & 64.5 & 79.5 \\
\hline 0.0 & 59.4 & 44.3 \\
\hline 0.0 & 48.2 & 44.3 \\
\hline 0.0 & 43.6 & 50.0 \\
\hline 0.0 & 13.0 & 50.0 \\
\hline 0.0 & 47.1 & 50.0 \\
\hline 0.0 & 56.6 & 44.3 \\
\hline 0.0 & 39.3 & 44.3 \\
\hline 2.0 & 58.5 & 64.8 \\
\hline 2.5 & 59.1 & 68.5 \\
\hline 5.0 & 73.6 & 86.9 \\
\hline 0.0 & 55.5 & 50.0 \\
\hline 0.0 & 57.9 & 38.5 \\
\hline 3.0 & 76.7 & 72.1 \\
\hline 0.0 & 44.8 & 44.3 \\
\hline 0.0 & 60.2 & 50.0 \\
\hline 0.0 & 46.5 & 38.5 \\
\hline 0.0 & 36.7 & 44.3 \\
\hline 0.5 & 95.6 & 53.7 \\
\hline 0.0 & 61.6 & 44.3 \\
\hline 2.0 & 61.9 & 64.8 \\
\hline 0.0 & 34.1 & 50.0 \\
\hline 1.0 & 41.4 & 57.4 \\
\hline 0.0 & 41.5 & 44.3 \\
\hline 4.5 & 85.6 & 83.2 \\
\hline 0.5 & 56.1 & 53.7 \\
\hline 1.0 & 68.8 & 57.4 \\
\hline 1.0 & 56.3 & 57.4 \\
\hline 0.0 & 36.5 & 44.3 \\
\hline 0.0 & 57.3 & 44.3 \\
\hline 1.0 & 59.0 & 57.4 \\
\hline 0.0 & 41.3 & 44.3 \\
\hline 0.0 & 55.2 & 44.3 \\
\hline 0.0 & 73.0 & 50.0 \\
\hline 0.0 & 43.4 & 50.0 \\
\hline 5.0 & 73.2 & 86.9 \\
\hline 0.0 & 48.8 & 44.3 \\
\hline 3.0 & 56.7 & 72.1 \\
\hline 0.0 & 38.6 & 50.0 \\
\hline 2.0 & 74.7 & 64.8 \\
\hline 2.0 & 69.5 & 64.8 \\
\hline 6.0 & 98.7 & 94.3 \\
\hline 0.0 & 72.0 & 44.3 \\
\hline 0.0 & 47.0 & 44.3 \\
\hline 0.0 & 54.5 & 44.3 \\
\hline 0.0 & 52.6 & 38.5 \\
\hline
\end{tabular}




\begin{tabular}{|c|c|c|c|c|}
\hline p-quaterphenyl & 4 & 0.0 & 66.2 & 38.5 \\
\hline prednisolone & 1 & 0.5 & 75.8 & 53.7 \\
\hline pregnenolone acetate & 1 & 1.0 & 64.8 & 57.4 \\
\hline progesterone & 1 & 0.0 & 66.8 & 50.0 \\
\hline propanal & 1 & 0.5 & 50.1 & 53.7 \\
\hline propane & 1 & 0.0 & 41.2 & 50.0 \\
\hline propene & 1 & 0.0 & 33.2 & 50.0 \\
\hline propionic acid & 1 & 0.5 & 42.2 & 53.7 \\
\hline propionitrile & 1 & 0.0 & 37.6 & 50.0 \\
\hline propyl 4-aminobenzoate & 1 & 3.0 & 59.2 & 72.1 \\
\hline propyl 4-hydroxybenzoate & 1 & 3.0 & 75.8 & 72.1 \\
\hline propyl N-phenyl carbamate & 1 & 4.0 & 63.7 & 79.5 \\
\hline propylcyclopentane & 1 & 3.5 & 64.4 & 75.8 \\
\hline propylene carbonate & 1 & 1.5 & 44.1 & 61.1 \\
\hline propylene oxide & 1 & 0.0 & 40.7 & 50.0 \\
\hline p-terphenyl & 4 & 0.0 & 77.0 & 38.5 \\
\hline p-toluic acid & 2 & 0.0 & 50.2 & 44.3 \\
\hline p-toluidine & 2 & 0.0 & 56.5 & 44.3 \\
\hline p-xylene & 4 & 0.0 & 59.8 & 38.5 \\
\hline pyrazine & 4 & 0.0 & 39.5 & 38.5 \\
\hline pyrazole & 1 & 0.0 & 41.4 & 50.0 \\
\hline pyrene & 4 & 0.0 & 43.4 & 38.5 \\
\hline pyridine & 2 & 0.0 & 35.8 & 44.3 \\
\hline \multicolumn{5}{|c|}{ pyromellitic dianhydride $1,2,5,6$-benzenetetracarboxylic } \\
\hline dianhydride & 4 & 0.0 & 28.4 & 38.5 \\
\hline pyrrole & 2 & 0.0 & 31.7 & 44.3 \\
\hline pyrrolidine & 1 & 2.0 & 42.4 & 64.8 \\
\hline quinazoline & 1 & 0.0 & 52.8 & 50.0 \\
\hline quinoline & 1 & 0.0 & 41.5 & 50.0 \\
\hline quinoxaline & 2 & 0.0 & 38.6 & 44.3 \\
\hline S-(+)-4-isobutyl-a-methylphenyl acetic acid & 1 & 3.0 & 57.5 & 72.1 \\
\hline \multicolumn{5}{|c|}{ S-(3,4-dihydro-4-oxobenzo[d]-[1,2,3]-triazin-3-ylmethyl) } \\
\hline O,O-diethyl phoshorodithioate & 1 & 5.5 & 78.3 & 90.6 \\
\hline \multicolumn{5}{|c|}{ S-(3,4-dihydro-4-oxobenzo[d][1,2,3]-triazin-3-ylmethyl) } \\
\hline O,O-dimethyl phoshorodithioate & 1 & 3.5 & 80.4 & 75.8 \\
\hline S-2,3,3-trichloroallyl diisopropylthiocarbamate & 1 & 5.5 & 88.5 & 90.6 \\
\hline \multicolumn{5}{|l|}{ S-2,3-dihydro-5-methoxy-2-oxo-1,3,4-thiadiazol- } \\
\hline 3-ylmethyl-O,O-dimethyl phosphorodithioate & 1 & 4.5 & 90.6 & 83.2 \\
\hline sebacic acid & 1 & 8.0 & 101.0 & 109.1 \\
\hline spiropentane & 4 & 0.0 & 38.7 & 38.5 \\
\hline$ß, \beta$-binaphthyl & 2 & 0.0 & 84.3 & 44.3 \\
\hline B-chloroacetic acid & 1 & 0.5 & 36.7 & 53.7 \\
\hline ß-chloroacetic acid & 1 & 0.5 & 42.3 & 53.7 \\
\hline B-naphthol & 1 & 0.0 & 47.7 & 50.0 \\
\hline B-naphthyl acetate & 1 & 1.0 & 58.6 & 57.4 \\
\hline B-propiolactone & 1 & 0.5 & 39.2 & 53.7 \\
\hline B-thiolactic acid & 1 & 0.5 & 58.1 & 53.7 \\
\hline styrene & 1 & 0.0 & 45.2 & 50.0 \\
\hline suberic acid & 1 & 6.0 & 69.4 & 94.3 \\
\hline succinic acid & 1 & 2.0 & 72.1 & 64.8 \\
\hline succinimide & 2 & 0.0 & 42.5 & 44.3 \\
\hline
\end{tabular}


succinonitrile terephthalyl dichloride tert-butyl alcohol tert-butyl amine tert-butyl bromide tert-butyl chloride tert-butyl mercaptan tert-butylbenzene tert-butylmethylsulfone testosterone

testosterone acetate testosterone formate testosterone propionate testosterone valerate tetra(methylthia)methane tetrachloroethene tetrachloro-o-xylene tetrachloro-p-xylene tetracontane tetracyanoethylene tetradecane tetradecanoic acid tetrafluoroethylene tetrahydrofuran tetramethylsuccinonitrile tetramethylurea tetramethysuccinic acid tetratriacontane tetrazole theophylline thiacyclobutane thiacyclohexane thiacyclopentane thianthrene

thiazole

thiophene

thiophenol

thiourea

thioxanthene 50-195

thioxanthone

thymine

thymol

toluene

tolyl vinyl sulfone

trans,cis-2,6-octadiene-1,8-dioic acid trans,trans-2,6-octadiene-1,8-dioic acid trans-1-(4-heptanoylphenyl)-4-heptylcyclohexane trans-1,2-cyclohexanediol trans-1,2-dimethylcyclohexane trans-1,3-dimethylcyclohexane trans-1,3-dimethylcyclopentane

$\begin{array}{rrrr}1 & 1.0 & 37.8 & 57.4 \\ 2 & 0.0 & 65.8 & 44.3 \\ 3 & 0.0 & 26.8 & 40.9 \\ 3 & 0.0 & 35.4 & 40.9 \\ 3 & 0.0 & 39.3 & 40.9 \\ 3 & 0.0 & 45.0 & 40.9 \\ 3 & 0.0 & 44.9 & 40.9 \\ 1 & 0.0 & 39.1 & 50.0 \\ 1 & 0.0 & 69.0 & 50.0 \\ 1 & 0.0 & 68.8 & 50.0 \\ 1 & 0.5 & 67.5 & 53.7 \\ 1 & 0.0 & 66.2 & 50.0 \\ 1 & 1.5 & 65.2 & 61.1 \\ 1 & 3.5 & 64.7 & 75.8 \\ 1 & 4.0 & 56.5 & 79.5 \\ 4 & 0.0 & 47.3 & 38.5 \\ 1 & 0.0 & 59.7 & 50.0 \\ 4 & 0.0 & 61.4 & 38.5 \\ 1 & 38.0 & 418.4 & 330.5 \\ 4 & 0.0 & 52.8 & 38.5 \\ 1 & 12.0 & 161.5 & 138.6 \\ 1 & 12.0 & 137.9 & 138.6 \\ 4 & 0.0 & 54.3 & 38.5 \\ 1 & 2.0 & 51.8 & 64.8 \\ 1 & 1.0 & 68.6 & 57.4 \\ 1 & 1.5 & 49.2 & 61.1 \\ 1 & 2.0 & 49.0 & 64.8 \\ 1 & 32.0 & 317.0 & 286.2 \\ 2 & 0.0 & 41.0 & 44.3 \\ 1 & 0.0 & 51.8 & 50.0 \\ 1 & 1.0 & 44.6 & 57.4 \\ 1 & 3.0 & 46.2 & 72.1 \\ 1 & 2.0 & 41.5 & 64.8 \\ 4 & 0.0 & 64.1 & 38.5 \\ 1 & 0.0 & 40.0 & 50.0 \\ 2 & 0.0 & 28.5 & 44.3 \\ 2 & 0.0 & 44.5 & 44.3 \\ 2 & 0.0 & 27.8 & 44.3 \\ 2 & 0.0 & 65.0 & 44.3 \\ 2 & 0.0 & 72.8 & 44.3 \\ 1 & 0.0 & 54.5 & 50.0 \\ 1 & 0.5 & 67.9 & 53.7 \\ 2 & 0.0 & 37.1 & 44.3 \\ 1 & 0.0 & 32.0 & 50.0 \\ 1 & 4.0 & 59.9 & 79.5 \\ 1 & 3.0 & 76.5 & 72.1 \\ 1 & 14.0 & 70.4 & 153.3 \\ 1 & 3.0 & 49.7 & 72.1 \\ 1 & 3.0 & 56.8 & 72.1 \\ 1 & 3.0 & 53.9 & 72.1 \\ 1 & 2.0 & 53.1 & 64.8\end{array}$




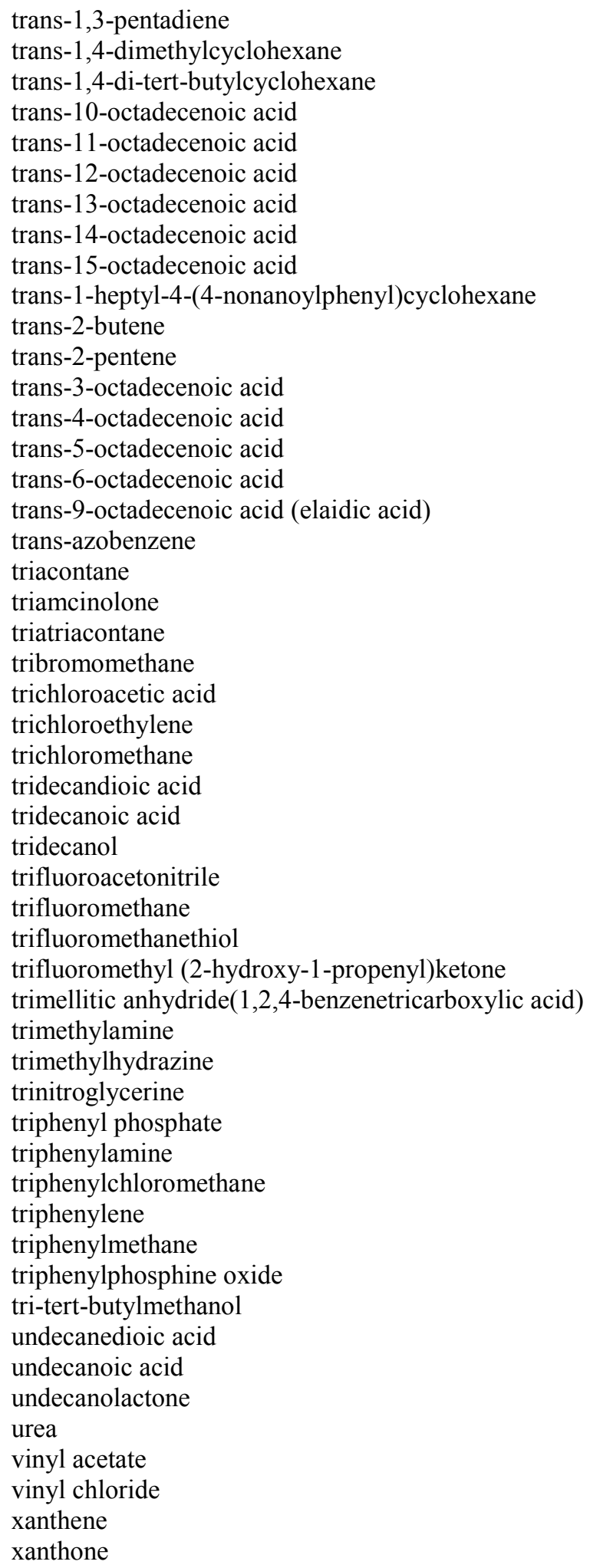

\begin{tabular}{|c|c|c|}
\hline 0.5 & 38.4 & 53.7 \\
\hline 3.0 & 52.2 & 72.1 \\
\hline 4.0 & 47.2 & 79.5 \\
\hline 14.0 & 179.5 & 153.3 \\
\hline 14.0 & 184.5 & 153.3 \\
\hline 14.0 & 174.5 & 153.3 \\
\hline 14.0 & 174.9 & 153.3 \\
\hline 14.0 & 174.5 & 153.3 \\
\hline 14.0 & 178.2 & 153.3 \\
\hline 16.0 & 92.6 & 168.1 \\
\hline 0.0 & 58.2 & 44.3 \\
\hline 1.0 & 62.8 & 57.4 \\
\hline 14.0 & 171.1 & 153.3 \\
\hline 14.0 & 167.8 & 153.3 \\
\hline 14.0 & 141.3 & 153.3 \\
\hline 14.0 & 184.5 & 153.3 \\
\hline 14.0 & 193.8 & 153.3 \\
\hline 0.0 & 66.1 & 38.5 \\
\hline 28.0 & 315.1 & 256.7 \\
\hline 0.5 & 78.4 & 53.7 \\
\hline 31.0 & 305.0 & 278.8 \\
\hline 0.0 & 39.4 & 40.9 \\
\hline 0.0 & 17.8 & 50.0 \\
\hline 0.0 & 44.8 & 50.0 \\
\hline 0.0 & 42.0 & 40.9 \\
\hline 11.0 & 116.9 & 131.2 \\
\hline 11.0 & 135.5 & 131.2 \\
\hline 12.0 & 148.1 & 138.6 \\
\hline 0.0 & 38.6 & 40.9 \\
\hline 0.0 & 34.4 & 40.9 \\
\hline 0.0 & 42.5 & 40.9 \\
\hline 0.5 & 36.4 & 53.7 \\
\hline 0.0 & 27.2 & 50.0 \\
\hline 0.0 & 41.9 & 50.0 \\
\hline 1.0 & 47.2 & 57.4 \\
\hline 6.5 & 76.6 & 98.0 \\
\hline 3.0 & 91.8 & 72.1 \\
\hline 0.0 & 62.2 & 35.1 \\
\hline 0.0 & 74.0 & 50.0 \\
\hline 0.0 & 52.5 & 35.1 \\
\hline 1.5 & 60.1 & 61.1 \\
\hline 0.0 & 56.1 & 40.9 \\
\hline 0.0 & 32.6 & 50.0 \\
\hline 9.0 & 103.0 & 116.4 \\
\hline 8.5 & 114.2 & 112.7 \\
\hline 7.0 & 58.0 & 101.7 \\
\hline 0.0 & 31.7 & 44.3 \\
\hline 1.0 & 46.8 & 57.4 \\
\hline 0.0 & 41.2 & 50.0 \\
\hline 0.0 & 51.4 & 44.3 \\
\hline 0.0 & 58.1 & 44.3 \\
\hline
\end{tabular}


Z-3-chloro-2-butenoic acid z-enantholactam
0.5

6.5
53.7

98.0 


\title{
APPENDIX D. Experimental and predicted Melting Points $\left(\mathrm{T}_{\mathrm{m}}\right)$ for 2230
}

\author{
organic compounds.
}

Name

(+)2-butanol

(+)a-(3-benzoylphenyl)propionic acid

(2,4,5-trichlorophenoxy)acetic acid

(2,4-dichlorophenoxy)acetic acid

(4-chloro-2-methylphenoxy)acetic acid

(4-chloro-o-tolyloxy)acetic acid

(d) 1,2-dibromoacenaphthene

(d) 1,2-dichloroacenaphthene

(d) 1,2-diphenyl-1,2-dihydroxyethane

(d) 2-(1-naphthoxy)propionamide

(D) 2-(2-chloro-3-methylphenoxy)propionic acid

(d) 2-(m-chlorophenoxy)propanoic acid

(d) 2-(o-chlorophenoxy)propanoic acid

(d) 2-(p-bromophenoxy)propanoic acid

(d) 2-(p-methoxyphenyl)propiophenone

(d) 2-(p-nitrophenoxy)propanoic acid

(d) 2,3-dibromo-1,4-butanediol

(d) 2-phenoxypropionic acid

(d) 3-(m-bromophenyl)-3-hydroxypropanoic acid

(d) 3-(m-chlorophenyl)-3-hydroxypropanoic acid

(d) 3-(m-fluorophenyl)-3-hydroxypropanoic acid

(d) 3-(o-fluorophenyl)-3-hydroxypropanoic acid

(d) 3-(p-bromophenyl)-3-hydroxypropanoic acid

(d) 3-(p-chlorophenyl)-3-hydroxypropanoic acid

(d) 3-(p-fluorophenyl)-3-hydroxypropanoic acid

(d) 3-hydroxy-3-phenylbutyric acid

(d) 3-hydroxy-3-phenylvaleric acid

(d) 3-phenyl-3-hydroxy-2,2-dimethylpropanoic acid

(d) 3-phenyl-3-hydroxypropanoic acid

(d) dimethyl diacetyltartrate

(d) dimethyl tartrate

(d) malic acid

(d) mandelic acid

(d) methylenebisthiopropionic acid

(d) m-fluoromandelic acid

(d) o-chloromandelic acid

(d) o-fluoromandelic acid

(d) p-chloromandelic acid

(d) p-fluoromandelic acid

(dl) 1,2-dibromoacenaphthene

(dl) 1,2-dichloroacenaphthene

(dl) 1,2-diphenyl-1,2-dihydroxyethane

(dl) 2-(1-naphthoxy)propionamide

(dl) 2-(2-chloro-3-methylphenoxy)propionic acid

(dl) 2-(m-chlorophenoxy)propanoic acid
$\mathbf{T}_{\mathrm{m}}(\mathbf{K})$

experimental

177.4

367.4

431.2

412.5

392.9

392.9

416.0

375.0

420.5

475.0

359.5

367.5

369.0

380.0

326.0

362.0

388.2

359.0

350.0

368.0

311.0

348.0

398.0

385.0

381.0

357.0

379.0

431.0

391.0

377.2

322.2

376.0

406.0

355.0

394.0

392.5

363.0

394.0

426.0

397.0

339.0

393.0

445.0

391.5

386.0 predicted

194.5

409.1

412.6

396.7

393.1

393.1

438.6

401.6

398.8

471.2

398.3

386.0

386.0

401.3

353.1

423.2

342.6

370.1

415.9

400.7

385.1

385.1

415.9

400.7

385.1

394.2

394.7

413.4

384.8

270.4

290.0

425.5

380.9

382.7

381.2

398.8

381.2

398.8

381.2

438.6

401.6

398.8

471.2

398.3

386.0 
(dl) 2-(o-chlorophenoxy)propanoic acid

386.0

(dl) 2-(p-bromophenoxy)propanoic acid

388.0

401.3

(dl) 2-(p-methoxyphenyl)propiophenone

353.1

(dl) 2-(p-nitrophenoxy)propanoic acid

353.0

423.2

(dl) 2,3-dibromo-1,4-butanediol

411.4

342.6

(dl) 2-phenoxypropionic acid

363.2

370.1

(dl) 3-(m-bromophenyl)-3-hydroxypropanoic acid

388.0

415.9

(dl) 3-(m-chlorophenyl)-3-hydroxypropanoic acid

349.0

400.7

(dl) 3-(m-fluorophenyl)-3-hydroxypropanoic acid

340.0

385.1

(dl) 3-(o-fluorophenyl)-3-hydroxypropanoic acid

290.0

385.1

(dl) 3-(p-bromophenyl)-3-hydroxypropanoic acid

342.0

415.9

(dl) 3-(p-chlorophenyl)-3-hydroxypropanoic acid

371.0

400.7

(dl) 3-(p-fluorophenyl)-3-hydroxypropanoic acid

385.1

(dl) 3-hydroxy-3-phenylbutyric acid

362.0

394.2

(dl) 3-hydroxy-3-phenylvaleric acid

330.0

394.7

(DL) 3-methylnonane

394.0

238.7

(dl) 3-phenyl-3-hydroxy-2,2-dimethylpropanoic acid

188.5

413.4

(dl) 3-phenyl-3-hydroxypropanoic acid

407.0

384.8

(DL) 4-methylnonane

366.0
174.7

238.7

(dl) dimethyl diacetyltartrate

355.2

270.4

(dl) dimethyl tartrate

360.2

(dl) malic acid I

402.0

290.0

(dl) malic acid II

396.0

425.5

(dl) mandelic acid

392.0

425.5

(dl) menthol

301.2

(dl) methylenebisthiopropionic acid

429.0

380.9

231.6

382.7

(dl) m-fluoromandelic acid

370.0

381.2

(dl) o-chloromandelic acid

358.5

398.8

(dl) o-fluoromandelic acid

390.0

381.2

(dl) p-chloromandelic acid

394.0

398.8

(dl) p-fluoromandelic acid

403.0

381.2

(1) menthol

316.2

231.6

[(benzoylamino)oxy] acetic acid

416.9

416.9

1-(4-chlorophenoxy)-3,3-dimethyl-(1H,1,2,4-triazol-1-yl)-2-butanone

351.4

399.4

1-(methylamino)-9,10-anthracenedione

443.2

1 -(o-chlorophenyl)thiourea

1,1-(2,2,2-trichloroethylidene)bis(4-chlorobenzene)

413.5

477.9

350.4

1,1'-(2,2,2-trichloroethylidene-bis(4-methoxy)benzene

382.1

371.1

$360.6 \quad 373.3$

$331.6 \quad 288.0$

1,1 '-(2,2-dichloroethylidenebis(4-chlorobenzene)

382.1

341.9

1,1-(di-p-chlorophenyl)-2-nitropropane

354.3

322.0

1,1,1,3-tetrachloropropane

237.7

311.1

1,1,1-trichloro-3,3,3-trifluoropropane

232.7

274.0

1,1,1-trichloroethane

240.1

241.3

1,1,1-trifluoro-3,3-dichloropropane

182.2

205.8

216.0

1,1,1-trifluoroethane

179.4

107.8

1,1,1-trifluoro-n-[2-methyl-4-(phenylsulphonyl)phenyl]methane sulfonamide

161.9

427.2

1,1,1-trinitroethane

418.4

266.6

251.4

216.6

1,1,2,2-tetrachloroethane

299.7

230.8

228.4

1,1,2-trichloroethane

244.0

226.8 
1,1,2-trifluoro-1,2,2-trichloroethane

215.1

1,1,3,3-tetraethylurea

236.9

283.4

1,1,3-trimethylurea

322.6

1,10-decanediol

1,12-benzoperylene

253.0

1,1-bis(4-chlorophenyl)-2-nitrobutane

470.7

1,1-dichloro-2,2-bis(4-chlorophenyl)ethylene

329.2

1,1-dichloroethane

381.5

1,1-dichloroethene

159.5

1,1-dicyclohexyldodecane

345.5

554.2

330.3

360.4

176.2

156.2

1,1-diethylurea

150.9

335.4

1,1-difluoro-1-chloroethane

300.6

392.3

1,1-dimethyl-3-phenylurea

342.3

124.6

1,1-dimethylcyclohexane

386.4

1,1-dimethylcyclopentane

404.8

183.6

1,1-dimethylurea

239.8

168.5

1,1-diphenyldodecane

1,2,3,4,5,6,7,8-octahydroanthracene

1,2,3,4,5-pentahydroxypentane (D-Arabitol)

203.7

$\begin{array}{ll}454.0 & 390.3 \\ 281.4 & 343.9\end{array}$

$345.4 \quad 347.8$

$379.4 \quad 382.0$

1,2,3,4,5-pentahydroxypentane (Ribitol)

$374.7 \quad 382.0$

1,2,3,4,5-pentahydroxypentane (Xylitol)

365.7

382.0

1,2,3,4-tetracarbomethoxybenzene

404.7

1,2,3,4-tetracarbomethoxynaphthalene

423.7

1,2,3,4-tetrachlorobenzene

320.0

389.6

1,2,3,4-tetrafluorobenzene

233.3

1,2,3,4-tetrahydronaphthlene

237.4

1,2,3,4-tetrahydroquinoline

290.0

1,2,3,4-tetrahydroxybutane

396.0

434.0

1,2,3,4-tetramethylbenzene

265.4

1,2,3,5-tetracarbomethoxybenzene

389.2

1,2,3,5-tetrachlorobenzene

323.9

1,2,3,5-tetrafluorobenzene

226.9

248.6

308.5

217.4

279.3

297.6

353.5

287.0

389.6

308.5

192.4

287.0

1,2,3,6,7,8-hexahydropyrene

407.7

483.4

1,2,3-tribromopropane

289.4

300.2

1,2,3-tricarbomethoxybenzene

375.7

339.7

1,2,3-tricarbomethoxynaphthalene

362.7

326.9

404.0

1,2,3-trichlorobenzene

407.2

285.2

1,2,3-trihydroxybenzene

466.9

1,2,3-trihydroxypropane

293.0

325.2

1,2,3-trimethylbenzene

247.8

269.2

1,2,4,5,8,9-tribenzopyrene

608.0

622.3

1,2,4,5-tetrabromobenzene

453.1

457.1

1,2,4,5-tetracarbomethoxybenzene

416.7

389.6

1,2,4,5-tetracarbomethoxynaphthalene

434.0

1,2,4,5-tetrachloro-3-nitrobenzene

438.2

341.7

1,2,4,5-tetrachlorobenzene

373.3

354.5

1,2,4,5-tetrafluorobenzene

421.2

249.9

1,2,4,5-tetramethylbenzene

277.0

329.9

1,2,4-triazole

352.4

350.5

1,2,4-tricarbomethoxynaphthalene

393.7

404.0

1,2,4-trichloro-5-((4-chlorophenyl)sulfonyl)benzene

419.9

450.0

1,2,4-trimethylbenzene

229.3

238.2 
1,2,5,6-tetracarbomethoxynaphthalene

434.0

1,2,5-tricarbomethoxynaphthalene

470.2

404.0

1,2,6,7-tetracarbomethoxynaphthalene

363.0

434.0

1,2,6-tricarbomethoxynaphthalene

407.2

404.0

1,2,7,8-tetrahydroxyoctane

416.7

368.7

1,2,7-tricarbomethoxynaphthalene

352.2

404.0

1,2,8-tricarbomethoxynaphthalene

427.2

404.0

1,2:3,4-dibenzanthracene

366.7

534.5

1,2:3,4-dibenzopyrene

553.5

1,2:4,5-dibenzopyrene

501.2

480.1

1,2:5,6-dibenzanthracene

520.2

480.1

1,2-benzacenaphthene (fluoranthene)

544.2

534.5

1,2-benzanthracene

383.4

1,2-benzofluorene

434.3

338.9

1,2-benzopyrene

462.8

402.5

380.8

1,2-bromochlorobenzene

454.4

462.7

1,2-bromoiodobenzene

260.6

251.6

1,2-butadiene

294.2

290.6

1,2-chloronitrobenzene

136.9

172.1

1,2-diamino-2-methylpropane

280.0

1,2-diaminopropane

308.2

267.4

1,2-dibenzoylethane

256.1

270.9

1,2-dibromo-1,1-difluoroethane

236.5

388.0

1,2-dibromobenzene

418.6

224.9

1,2-dibromoethane

206.3

306.6

1,2-dibromotetrafluoroethane

265.6

283.0

184.2

1,2-dicarbomethoxybenzene

162.8

295.9

1,2-dicarbomethoxynaphthalene

274.2

367.3

1,2-dichlorobenzene

358.2

262.0

1,2-dichloroethane

1,2-dichloropropane

256.5

237.2

237.0

1,2-dichloro-tetrafluoroethane

172.7

208.1

1,2-dicyanobenzene

180.6

178.7

1,2-difluoro-2,2-dichloroethane

414.1

402.3

1,2-difluorobenzene

163.0

225.3

1,2-dihydro-6-neopentyl-2-oxonicotinic acid

226.0

216.5

1,2-dihydroxybenzene

469.2

469.2

1,2-diiodobenzene

376.9

374.5

1,2'-dinaphthylmethane

296.6

350.2

$369.6 \quad 406.4$

1,2-dinitrobenzene

396.1

370.7

1,2-diphenyl-2-(N-piperidinyl)-1-ethanone

349.2

351.5

1,2-diphenylethane

324.3

283.6

1,2-pentadiene

135.9

92.1

375.5

358.4

1,3,5,5-tetranitro-1,3-diazacyclohexane

430.0

429.7

1,3,5,7-tetroxane

385.0

306.0

1,3,5-tri-a-naphthylbenzene

472.0

494.8

1,3,5-tricarbomethoxynaphthalene

402.7

404.0

1,3,5-trichloro-2,4,6-trifluorobenzene

335.0

361.1

1,3,5-trichlorobenzene

336.7

359.2

1,3,5-trimethylbenzene

228.4

339.0

1,3,5-trinitro-1,3,5-triazacyclohexane

478.2

380.3

434.0

1,3,5-trinitrobenzene

396.8 
1,3,5-trinitroso-1,3,5-triazacyclohexane

394.9

1,3,5-trioxane

376.0

276.5

1,3,5-triphenylbenzene

333.4

437.6

1,3,6-trimethyluracil

446.0

437.6
374.8

1,3,7-tricarbomethoxynaphthalene

384.5

1,3,7-trichlorodibenzodioxin

446.7

404.0

1,3,8-tricarbomethoxynaphthalene

397.4

1,3-bromochlorobenzene

421.7

404.0

1,3-bromoiodobenzene

388.2

251.6

1,3-butadiene

252.0

290.6

1,3-cyclohexadiene

282.5

179.4

1,3-dibromobenzene

164.2

188.2

1,3-dibromopropane

161.0

306.6

1,3-dibutylurea

266.3

280.8

1,3-dicarbomethoxybenzene

346.9

368.1

1,3-dicarbomethoxynaphthalene

341.2

295.9

1,3-dichlorobenzene

378.7

367.3

1,3-dicyanopropane

248.4

262.0

1,3-diethylurea

244.2

298.4

383.4

355.9

1,3-difluorobenzene

216.5

1,3-dihydroxybenzene

204.0

383.1

1,3-diiodobenzene

382.6

350.2

1,3-dimethyluracil

307.4

331.5

1,3-dimethylurea

398.0

345.4

1,3-dinitro-1,3-diazacycloheptane

381.0

374.0

403.3

1,3-dinitro-1,3-diazacyclohexane

354.0

353.9

1,3-dinitro-1,3-diazacyclopentane

410.0

377.3

1,3-dinitro-5-nitroso-1,3,5-triazacyclohexane

446.0

421.0

1,3-dinitrobenzene

363.2

1,3-dioxolane

175.9

328.1

1,3-diphenylacetone

307.2

1,3-diphenylurea

512.0

1,3-dithiane

327.2

1,3-nitrochlorobenzene

317.6

1,4,5,8-tetracarbomethoxynaphthalene

477.2

1,4,5-tricarbomethoxynaphthalene

1,4,6-tricarbomethoxynaphthalene

402.2

1,4-[bis[(4-methylphenyl)amino]-9,10-anthracenedione

409.2

491.2

1,4-bis(diphenylphosphino)butane

405.9

425.1

241.3

319.6

490.1

317.4

280.0

434.0

404.0

404.0

502.6

399.2

1,4-bis(phenylglyoxaloyl)benzene

464.2

408.7

1,4-bis-[4-(4'-n-butylbiphenyl)]butane

337.8

397.8

1,4-bromochlorobenzene

1,4-bromoiodobenzene

363.3

1,4-cyclohexadiene

224.0

284.3

328.4

244.1

$348.2 \quad 276.0$

1,4-diamino-2-methoxyanthraquinone

515.2

550.0

1,4-diaminoanthraquinone

484.2

626.1

1,4-dibromobenzene

360.1

352.4

1,4-dicarbomethoxybenzene

413.8

295.9

1,4-dicarbomethoxynaphthalene

340.2

367.3

1,4-dichloro-2,5-dimethoxybenzene

316.1

1,4-dichlorobenzene

301.1

1,4-dihydroxybenzene

326.0

440.3 
1,4-dihydroxybutane

307.9

1,4-diiodobenzene

293.6

402.5

1,4-dimethylnaphthalene

331.0

1,4-dinitrobenzene

279.9

1,4-dioxane

1,4-dioxane-2,5-dione

248.9

1,4-di-tert-butylbenzene

379.4

1,4-dithiane

446.7

321.2

1,4-nitrochlorobenzene

317.4

1,4-pentadiene

1,5-cyclooctanedione

356.2

341.5

384.6

316.3

1,5-dicarbomethoxynaphthalene

354.6

58.5

124.3

283.3

1,5-dichloro-3-oxapentane

341.2

367.3

1,5-dimethyltetrazole

226.5

264.9

1,5-dinitro-3-nitroso-1,3,5-triazacycloheptane

349.0

273.0

1,5-pentanediol

440.0

411.2

1,6-bis-[4-(4'-ethylbiphenyl)]hexane

248.0

316.4

1,6-dicarbomethoxynaphthalene

422.2

387.2

1,6-hexanediol

1,7-diacetoxy-2,4,6-trinitro-2,4,6-triazaheptane

371.8

367.3

$314.7 \quad 323.5$

1,7-dicarbomethoxynaphthalene

422.5

435.2

1,7-heptanediol

363.2

367.3

1,8-bis-(4-biphenyl)octane

295.2

329.4

1,8-bis-[4-(4'-ethylbiphenyl)]butane

415.2

454.2

425.9

1,8-bis-[4-(4'-ethylbiphenyl)]octane

413.2

1,8-bis-[4-(4'-n-butylbiphenyl)]octane

414.2

397.3

397.8

1,8-dimethylnaphthalene

398.2

1,8-octanediol

336.3

331.0

1,9-nonanediol

332.8

334.5

1-[3,5-di-tert-butyl-4-hydroxyphenyl]-5-hexyn-1-one

319.6

338.9

$10 \mathrm{H}$-phenothiazine

342.2

10-nonadecanone

458.2

425.5

497.7

305.5

10-octadecynoic acid

330.0

307.9

11-cyclohexyleicosane

319.0

340.3

11-heneicosanone

269.9

313.1

11-n-decylheneicosane

336.7

328.9

11-octadecynoic acid

282.3

307.9

11-phenyleicosane

320.0

335.8

12-octadecynoic acid

294.3

320.0

307.9

12-tricosanone

342.2

13-octadecynoic acid

322.0

319.5

14-octadecynoic acid

337.0

307.9

16-octadecynoic acid

17-alpha-hydroxyprogesterone caproate

347.0

393.2

438.0

340.0

307.9

323.1

418.5

17-octadecynoic acid

542.3

401.0

1-8-naphthalic anhydride

386.8

344.0

1a,2a,3ß,4a,5a,6ß-hexachlorocyclohexane

319.2

455.5

1 -acetoxynaphthalene

337.0

313.2

1-acetyl-2-naphthol

458.2

311.8

393.0

1-amino-4-hydroxy-2-phenoxy-9,10-anthracenedione

592.7

1 -aminoanthraquinone

509.7

1-aminopropane

524.2

188.4

205.7 
1-benzoyl-2-naphthol

414.1

1-bromo-2-chloro-1,1,2-trifluoroethane

1-bromo-2-chloroethane

1-bromobutane

1-bromodocosane

1-bromoheptane

1-bromohexane

1-bromonaphthalene

1-bromononane

1-bromooctane

1-bromopentane

1-bromotricosane

1-bromoundecane

1-butene

1-butyne

1-chloro-2-(2,2,2-trichloro-1-(4-chlorophenyl)ethyl)benzene

1-chloro-2-(2,2-dichloro-1-(4-chlorophenylethenyl)benzene

1-chloro-2,2-(bis-(4-chlorophenyl)ethylene

1-chlorodibenzodioxin

1-chloronaphthalene

1-cis-3-pentadiene

1-cyclohexyl-1-phenyldodecane

1-decanethiol

1-decanol

1-decene

1-deoxy-D-glucopyranose

1-docosanol

1-dodecene

1H-1,2,4-triazol-3-amine

1-heptanethiol

1-heptanol

1-heptene

1-hexacosanol

1-hexadecanol

1-hexadecene

1-hexanethiol

1-hexanol

1-hexene

1-iodonaphthalene

1-methoxy-2-(2,2,2-tricloro-1-(4-methoxyphenyl)ethyl)benzene

1-methyl-7-isopropylphenanthrene

1-methyl-9H-pyrido[3,4-b]indole

1-methylcyclohexanol

1-methylcyclopentanol

1-methylnaphthalene

1-methyltetrazole

1-naphthaleneacetamide

1-naphthaleneacetic acid

1-naphthoic acid

1-naphthyl benzoate

1-naphthyl methylcarbamate

1-naphthylamine

1-nitronaphthalene $\begin{array}{ll}414.1 & 409.9\end{array}$

$256.4 \quad 251.3$

$160.4 \quad 235.9$

$317.2 \quad 333.4$

$214.4 \quad 277.6$

$188.1 \quad 266.3$

$271.4 \quad 301.6$

$243.2 \quad 295.3$

$218.2 \quad 287.1$

$185.1 \quad 252.6$

$339.6 \quad 348.2$

$263.3 \quad 308.5$

$87.8 \quad 83.6$

$147.4 \quad 134.2$

$345.8 \quad 371.1$

$349.8 \quad 337.6$

$337.9 \quad 347.0$

$378.2 \quad 356.3$

$270.7 \quad 281.9$

$132.4 \quad 171.5$

$275.8 \quad 355.5$

$247.9 \quad 276.5$

$280.1 \quad 307.3$

$206.9 \quad 226.4$

$403.2 \quad 415.0$

$345.2 \quad 336.1$

$237.9 \quad 241.2$

$428.3 \quad 420.7$

$229.9 \quad 245.1$

$240.4 \quad 283.8$

$154.3 \quad 175.9$

$351.7 \quad 344.0$

$322.2 \quad 318.6$

$277.5 \quad 273.2$

$192.6 \quad 230.8$

$225.8 \quad 273.1$

$133.4 \quad 151.7$

$\begin{array}{ll}280.0 & 320.9\end{array}$

$347.6 \quad 373.3$

$369.0 \quad 333.4$

$509.9 \quad 465.8$

$299.2 \quad 218.0$

$310.2 \quad 206.9$

$242.7 \quad 277.1$

$315.0 \quad 257.2$

$455.5 \quad 446.7$

$405.3 \quad 394.1$

$435.2 \quad 455.3$

$329.2 \quad 413.9$

$416.3 \quad 383.8$

$323.2 \quad 331.6$

$329.9 \quad 330.0$ 
1-nonanethiol 267.7

1-nonene

$191.6 \quad 212.3$

1-octanethiol

224.0

257.2

1 -octene

171.5

195.7

1-pentadecanol

316.6

314.7

1-pentanethiol

197.5

213.5

1-pentanol

195.6

260.1

1-pentene

107.9

121.8

1-propanethiol

160.0

165.7

1-propanol

148.8

224.3

1-tetradecanol

311.0

310.4

1-undecene

224.0

238.5

2-((4-chloro-6-(cyclopropylamino)-1,3,5-triazin-2-yl)amino-2-methylpropanenitrile438.5

542.3

2-(1,3-dioxolan-2-yl)phenyl methylcarbamate

387.2

468.8

2-(1'-cyclohexenyl)cyclohexanone

278.8

226.9

2-(1-methylethyl)phenyl methylcarbamate

369.3

319.8

2-(2,4,5-trichlorophenoxy)propanoic acid

450.6

417.8

2-(2,4-dichlorophenoxy)propanoic acid

$389.2 \quad 401.9$

2-(3,4-dichlorophenyl)-4-methyl-1,2,4-oxadiazolidine-3,5-dione $\quad 396.3 \quad 396.3$

2-(3-hydroxy-2-quinolinyl)-1H-indene-1,3(2H)-dione $\quad 539.2 \quad 564.6$

2-(4-chloro-2-methylphenoxy)propanoic acid

$366.2 \quad 398.3$

2-(6-methoxy-2-naphthyl)propionic acid

$439.2 \quad 399.0$

2-(dimethylamino)-1,2-diphenylethanone

334.2

2-(docosanoxy)ethanol

335.9

340.1

2-(hexadecyloxy)ethanol

318.5

336.2

2,11-dicyclohexyldodecane

300.6

320.8

2,2,2-trinitroethanol

344.9

337.8

2,2,2-trinitroethyl 4,4,4-trinitrobutyrate

366.5

343.8

2,2,2-trinitroethyl-4,4-dinitropentanoate

366.7

476.0

2,2',3,3',4,5,5',6,6'-nonachlorobiphenyl

455.8

2,2',3,3',5,5',6,6'-octachlorobiphenyl

433.8

395.4

2, ', 3 , 3',5,5'-hexachlorobiphenyl

424.9

2,2',3,3',6,6'-hexachlorobiphenyl

385.2

2,2,3,3-tetramethylbutane

373.9

263.4

2,2,3,3-tetramethylpentane

247.7

2,2,3-trimethylbutane

386.7

322.0

2,2,4,4-tetramethylpentan-3-ol

206.7

2,2,4,4-tetramethylpentane

350.1

2,2',4,5,5'-pentachlorobiphenyl

339.1

165.8

268.9

2,2,4-trimethylpentane

2,2,5,5-tetramethylhex-3-ene

250.6

355.8

329.0

367.2

542.1

596.3

438.6

472.3

418.0

368.5

277.0

190.5

542.8

352.7

317.9

397.4

376.8

217.4

201.8

228.5

2,2-bis-(4-cyanatophenyl)propane

355.8

2,2-bis(phenylthio)propane

468.0

375.2

2,2-bis-hydroxymethylpropanoic acid

239.3

415.1

2,2-dichloropropane

2,2-dicyanopropane

307.5

227.9

253.7

2,2-dimethoxy-1,2-diphenylethanone

338.5

293.7

2,2-dimethyl-1,3-dioxan

229.6

238.7

2,2-dimethyl-1,3-propanediol

403.2

366.5 
2,2-dimethyl-1-propanol $\quad 264.0$

2,2-dimethylbutane

2,2-dimethylheptane

$160.0 \quad 277.6$

2,2-dimethylpentane

$148.1 \quad 246.3$

2,2-dimethylpropane

$256.5 \quad 280.2$

2,2-dimethylpropanoic acid (pivalic acid)

$309.1 \quad 312.4$

2,2-dinitro-1,3-propanediol

$341.2 \quad 355.5$

2,2-dinitropropane

$324.5 \quad 204.5$

2,2-dinitropropanol

$366.7 \quad 289.7$

2,2-dinitropropyl-4,4-dinitropentanoate

$370.8 \quad 335.5$

2,2-dintropropyl-4,4,4-trinitrobutyrate

$368.2 \quad 353.9$

2,2'-methylenebis(3,4,6-trichlorophenol)

437.6

513.6

2,3,4,5,6-pentachlorobiphenyl

$397.6 \quad 449.1$

2,3,4,5,6-pentafluorotoluene

243.7

235.8

$363.9 \quad 376.8$

163.6

114.2

2,3,5,6-tetrachloro-2,5-cyclohexadiene-1,4-dione

567.2

605.8

2,3,5-tricarbomethoxynaphthalene

401.7

404.0

2,3,5-triiodobenzoic acid

503.8

537.7

2,3,6,7,10,11-hexakis(1-decynyl)triphenylene

314.2

358.4

2,3,6,7-tetracarbomethoxynaphthalene

458.2

434.0

2,3,6-tricarbomethoxynaphthalene

399.2

2,3,6-trichlorobenzoic acid

402.7

404.0

2,3,6-trichlorophenylacetic acid

2,3-benzofluorene

432.3

394.6

$\begin{array}{ll}489.7 & 380.8\end{array}$

2,3-dicarbomethoxynaphthalene

324.2

2,3-dichloro-1,4-naphthalenedione

367.3

$469.0 \quad 492.6$

2,3-dichlorophenol

330.0

302.2

2,3-dihydro-2,2-dimethyl-7-benzofuranol-3-one

440.6

412.1

2,3-dihydro-2,2-dimethylbenzofuran-7-yl methylcarbamate

426.2

412.9

2,3-dihydro-6-methyl-5-phenylcarbamoyl-1,4-oxathiin-4,4-dioxide

401.5

401.5

$493.0 \quad 441.6$

2,3-dimethyl-2,3-bis(phenylazo)butane

342.3

391.1

2,3-dimethyl-2,3-butanediol

316.2

312.3

2,3-dimethyl-2,3-diphenylbutane

392.0

340.8

2,3-dimethyl-2-butene

198.9

253.6

2,3-dimethylbenzoic acid

417.6

145.2

416.4

2,3-dimethylbutane

378.0

106.4

331.0

2,3-dimethylphenol

346.0

296.5

2,3-dimethylpyridine

258.6

238.4

2,3-dinitrophenol

417.0

376.4

329.8

343.9

2,3-dinitrotoluene

147.5

222.6

2,4,4-trimethyl-1-pentene

178.9

214.4

2,4,4-trimethyl-2-pentene

166.0

198.5

2,4,5,6-tetrachloro-1,3-benzenedicarbonitile

526.2

495.3

2,4,5-tribromostyrene

2,4,5-trichlorobiphenyl

340.3

342.0

$349.5 \quad 356.3$

$340.3 \quad 322.8$

2,4,5-trichlorophenol

240.7

254.5

2,4,5-trinitrotoluene

376.2

412.6

2,4,6,N-tetranitro-N-methyltoluidene

375.6

458.4 
2,4,6-N-tetranitroethylaniline $\quad 369.0$

494.6

2,4,6-tribromophenol

366.2

378.2

2,4,6-trichlorobiphenyl

$334.3 \quad 402.6$

2,4,6-trimethyl-1,3,5-trioxane

$285.7 \quad 359.7$

2,4,6-trimethylpyridine

229.0

254.3

2,4,6-trinitro-1,3-dimethylbenzene

455.4

2,4,6-trinitroresorcinol

454.8

428.5

2,4,6-trinitrotoluene

352.2

441.5

2,4,6-tri-tert-butylphenol

405.2

466.2

2,4,7-trinitrofluoren-9-one

449.2

460.7

2,4-dibromophenol

313.0

397.1

2,4-dichlorophenol

318.0

341.7

2,4-dichlorophenyl 4-nitrophenyl ether

302.2

$342.0 \quad 371.6$

2,4-dimethylpentane

$154.0 \quad 139.8$

2,4-dimethylpyridine

209.4

238.4

2,4-dimethylpyrrole

268.5

324.3

2,4-dimethylthiazole

222.9

269.7

2,4-dinitrochlorobenzene

325.2

348.7

2,4-dinitrophenol

388.0

376.4

2,4-dinitrotoluene

343.3

343.9

2,4-di-tert-butylthiazole

258.2

337.6

2,4-hexadiyne

341.7

182.4

2,5,8,11-tetraoxadodecane

229.3

236.1

2,5,8-trioxanonane

209.1

212.4

2,5-dibutoxy-1,4-benzoquinone

473.3

395.0

2,5-dichlorophenol

$331.0 \quad 302.2$

2,5-diethoxy-1,4-benzoquinone

459.3

393.2

2,5-dimethylaniline

279.0

292.6

2,5-dimethylphenol

348.0

296.5

2,5-dimethylpyridine

259.1

238.4

2,5-dimethylpyrrole

280.9

366.5

2,5-dimethyltetrazole

256.4

273.0

2,5-dimethylthiophene

210.6

251.6

2,5-di-n-heptadecyloxy-1,4-benzoquinone

395.3

395.5

2,5-di-n-heptyloxy-1,4-benzoquinone

406.2

2,5-di-n-hexadecyloxy-1,4-benzoquinone

394.2

2,5-di-n-hexyloxy-1,4-benzoquinone

412.1

391.6

2,5-dinitrophenol

381.0

395.3

395.9

2,5-di-n-nonadecyloxy-1,4-benzoquinone

376.4

2,5-di-n-nonyloxy-1,4-benzoquinone

396.2

395.8

2,5-di-n-octyloxy-1,4-benzoquinone

402.7

392.9

2,5-di-n-pentadecyloxy-1,4-benzoquinone

405.8

392.3

$393.5 \quad 395.0$

2,5-di-n-undecyloxy-1,4-benzoquinone

397.2

393.8

2,5-dipentoxy-1,4-benzoquinone

414.6

395.5

2,5-dipropoxy-1,4-benzoquinone

460.8

394.2

2,6-dichloro-4-benzenamine

467.2

341.4

2,6-dichloro-4-nitroaniline

466.8

410.4

2,6-dichlorobenzonitrile

417.2

355.4

2,6-dichlorobiphenyl

307.9

379.3

2,6-dichlorophenol

340.0

337.2

2,6-diisopropylphenol

292.8

274.0

2,6-dimethylnaphthalene

331.0

2,6-dimethylphenol

383.3

318.9

335.1 
2,6-dimethylpyridine

2,6-dinitrochlorobenzene

2,6-dinitro-N,N-dipropyl-4-(trifluoromethyl)benzenamine

2,6-dinitrophenol

2,6-dinitrotoluene

327.5

343.9

2,6-di-tert-butyl-4-methoxyphenol

440.1

2,6-di-tert-butyl-4-methylphenol

374.4

2,6-di-tert-butylphenol

343.7

411.3

2,7-dicarbomethoxynaphthalene

310.7

395.4

2,7-dimethylnaphthalene

410.2

367.3

2-[(trichloromethyl)thio]-1H-isoindole-1,3(2H)-dione

368.8

331.0

2-[[3-(trifluoromethyl)phenyl]amino]-3-pyridinecarboxylic acid

454.2

455.9

2-[[4-chloro-6-(ethylamino)-1,3,5-triazin-2-yl]amino]-2-methylpropanenitrile

2-[bis(2-chloroethyl)amino]tetrahydro-2H-1,3,2-oxazophosphorine-2-oxide

2-acetoxynaphthalene

342.2

322.6

2-acetyl-1-naphthol

371.8

311.8

418.9

2-acetylamino-9-[(2-hydroxyethoxy)methyl]-1,9-dihydro-6H-purin-6-one

490.2

2-acetylamino-9-[(2-hydroxyethoxy)methyl]-9H-purine

454.2

2-amino-2-hydroxymethylpropane-1,3-diol

407.5

384.0

2-amino-9-[(2-acetoxyethoxy)methyl]-1,9-dihydro-6H-purin-6-one

515.2

2-amino-9-[(2-hydroxyethoxy)methyl]-1,9-dihydro-6H-purin-6-one

528.2

2-amino-9-[(2-hydroxyethoxy)methyl]-9H-purine

462.2

2-aminobenzoic acid

2-aminobiphenyl

417.8

322.3

178.0

2-benzoyl-1-naphthol

343.9

2-bromo-2-chloro-1,1,1-trifluoroethane

154.7

2-bromobutane

2-bromonaphthalene

2-bromopropane

160.3

329.0

184.1

203.9

2-bromothiophene

133.0

2-butanol

184.7

2-butanone

186.5

2-butyne

240.9

2-carbomethoxynaphthalene

350.2

358.8

261.6

2-chloro-2-nitropropane

337.8

370.3

541.2

476.3

404.8

352.2

511.6

509.3

434.8

403.2

364.8

143.9

435.8

193.6

186.0

301.6

154.5

231.3

136.8

194.5

176.0

131.1

321.1

305.4

253.3

325.2

2-chloro-9-(3-dimethylaminopropylidene)-10-thioxanthene

320.3

2-chloroanthraquinone

483.0

485.9

379.4

2-chlorobenzoic acid

413.4

315.1

2-chlorobiphenyl

304.9

362.2

356.3

2-chloroethylphosphonic acid

347.9

347.9

2-chloro-n-(2,6-diethylphenyl)-n-(methoxymethyl)acetamide

315.9

271.9

2-chloronaphthalene

2-chloro-N-isopropyl N-phenylacetamide

332.0

281.9

351.4

271.5

294.2

281.6

2-chlorophenol

351.3

283.0

156.0

138.1

2-chlorothiophene

201.3

211.5 
2-cyano-2-methylpropane

2-fluorenyl-2-methyl-1,3-cyclopentanedione

2-heneicosanone

2-hexadecanoyloxy-1,3-bis-(9-cis-octadecenoyloxy)propane

2-hydroxybenzoic acid

2-hydroxymethyl-2-methyl-1,3-propanediol

423.5

2-iodobenzoic acid

2-iodonaphthalene

2-isopropoxyphenyl N-methylcarbamate

418.4

320.9

2-methoxy-4H-1,3,2-benzodioxaphosphorin 2-sulfide

332.9

2-methyl-1,3-butadiene

322.2

138.2

2-methyl-1-butene

2-methyl-1-phenyl-2-(N-piperidinyl)-1-propanone

126.7

2-methyl-1-propanol

332.1

195.4

2-methyl-2-(methylsulfonyl)propanal oxime

373.9

2-methyl-2(methylthio)propionaldehyde O-methylcarbamoyloxime

353.7

2-methyl-2-butanethiol

166.7

2-methyl-2-butanol

211.1

2-methyl-2-butene

149.1

2-methyl-2-diphenylmethyl-1,3-cyclopentanedione

393.3

2-methyl-2-nitro-1,3-propandiol

360.9

2-methyl-2-nitro-1-propanol

295.8

2-methyl-2-nitropropyl-4,4,4-trinitrobutyrate

357.8

2-methyl-4,6-dinitrophenol

392.2

2-methylbutane

2-methylcyclothiapentane

2-methyldecane

2-methylfuran

2-methylheptane

193.9

2-methylhexane

208.9

2-methylnaphthalene

189.4

2-methylnonane

164.2

277.1

2-methylpentane

154.9

$\begin{array}{ll}198.8 & 238.7 \\ 119.6 & 165.4\end{array}$

2-methylpiperidine

199.2

2-methylpyridine

2-methyltetrazole

269.4

2-methylthiazole

2-methylthiophene

2-naphthoic acid 
2-nitro-5-methylphenol

2-n-propyl-5-(4-bromophenyl)thiophene

$360.4 \quad 406.2$

2-octanone

252.9

255.3

2-pentadecanone $\quad 312.2$

302.3

2-pentanol

200.0

217.8

2-pentanone

196.3

203.0

2-phenylbenzimidazole

2-piperadone

342.3

341.3

2-propanethiol

142.6

98.0

2-propanol

185.2

2-pyrrolidone

299.0

164.2

2-sec-butyl-4,6-dinitrophenol

344.5

2-sec-butyl-4,6-dinitrophenyl 3-methylcrotonate

313.7

363.8

2-tetradecanone

341.3

371.1

2-undecanone

306.7

296.8

3-(1-methylethyl)-(1H)-2,1,3-benzothiadiazin-4(3H)-one 2,2-dioxide

290.5

285.6

3-(3,4-dichlorophenyl)-1,1-dimethylurea

412.5

3-(4-chlorophenyl)-1,1-dimethylurea

429.7

412.5

418.2

3-(5-(1,1-dimethylethyl)-1,3,4-thiadiazol-2-yl)-4-hydroxy-1-methyl-

3(n-decylamino)-1,2-propanediol

346.6

334.4

3(n-decyloxy)-1,2-propanediol

311.0

312.9

3(n-decylthio)-1,2-propanediol

311.9

321.4

3(n-dodecylamino)-1,2-propanediol

340.4

3(n-dodecyloxy)-1,2-propanediol

351.9

320.8

3(n-dodecylthio)-1,2-propanediol

323.0

328.5

3(n-heptylamino)-1,2-propanediol

325.5

343.2

3(n-heptyloxy)-1,2-propanediol

324.9

316.2

3(n-heptylthio)-1,2-propanediol

246.2

326.9

3(n-hexyloxy)-1,2-propanediol

292.5

272.9

310.6

3(n-hexylthio)-1,2-propanediol

290.8

322.0

3(n-nonylamino)-1,2-propanediol

343.2

349.5

3-(n-nonyloxy)-1,2-propanediol

297.2

325.6

3(n-octylamino)-1,2-propanediol

335.9

346.6

3(n-octyloxy)-1,2-propanediol

3(n-octylthio)-1,2-propanediol

296.1

321.2

3(n-tetradecylamino)-1,2-propanediol

331.2

$356.2 \quad 345.3$

3(n-tetradecyloxy)-1,2-propanediol

331.3

327.4

3(n-tetradecylthio)-1,2-propanediol

336.4

334.5

3(n-tridecylamino)-1,2-propanediol

354.9

343.0

3(n-tridecyloxy)-1,2-propanediol

324.2

324.3

3(n-tridecylthio)-1,2-propanediol 3(n-undecylamino)-1,2-propanediol

3(n-undecyloxy)-1,2-propanediol

348.8

337.5

311.7

317.0

3(n-undecylthio)-1,2-propanediol

317.4

325.1

3-(p-tolyl-4-sulfonyl)-1-butyl urea

404.8

382.0

3,3',4'4'-tetraaminodiphenyl ether

425.1

507.6

3,3',5,5'-tetra-tert-butyldiphenylmethane-4,4'-diol

447.7

422.7

3,3'-bis-(1-cyclohexylethyl)-5,5'-dimethyldiphenylmethane-2,2'-diol

400.7

432.8

292.2

318.9

3,3-diethylpentane

240.1 
3,3-dimethyl-1-(methylthio)-2-butanone O-methylcarbamoyloxime

3,3-dimethylpentane

3,3-dimethylpentanedioic anhydride

138.2

218.2

3,3'-di-tert-butyl-5,5'-dimethyl-2,2-dihydroxydiphenylmethane

396.2

365.5

3,4-benzophenanthrene

403.7

440.6

3,4-benzopyrene

334.7

402.5

3,4-dichloro-2-methoxybenzoic acid

454.2

409.5

3,4-dichlorophenol

412.5

412.4

3',4'-dichloropropionanilide

341.0

306.0

3,4-diethyl-3,4-bis(4-tert-butylphenyl)hexane

363.7

372.7

3,4-dimethylisoxazol 5-sulphanylamide

400.0

370.4

3,4-dimethylphenol

448.2

467.9

3,4-dimethylphenyl methylcarbamate

334.0

296.5

3,4-dimethylpyridine

350.8

353.7

3,4-dinitrophenol

262.7

238.4

3,4-dinitrotoluene

407.0

402.3

3,5,6-trichloro-2-pyridinol

329.5

343.9

3,5,6-trichloro-2-pyridinyloxyacetic acid

448.1

338.8

$423.3 \quad 425.0$

3,5-dibromo-4-hydroxybenzonitrile

464.0

475.2

3,5-dichlorobenzoic acid

459.3

481.2

3,5-dichloro-N-(1,1-dimethyl-2-propynyl)benzamide

428.4

409.7

3,5-dichlorophenol

341.0

345.8

3,5-diisopropylphenol

326.3

274.0

3,5-dimethyl-4-(dimethylamino)phenyl methylcarbamate

361.7

3,5-dimethylbenzoic acid

355.6

3,5-dimethylphenol

442.9

470.5

3,5-dimethylpyridine

336.8

335.1

3,5-dinitrobenzoic acid

266.9

269.4

3,6-dichloro-2-methoxybenzoic acid

480.4

522.1

3,6-dichloro-5-hydroxy-2-methoxybenzoic acid

386.7

409.8

412.4

3-amino-2,5-dichlorobenzoic acid

475.6

454.4

466.4

3-aminoacetophenone

371.2

3-aminobenzoic acid

452.9

344.4

3-bromopentane

3-chloro-1,1,1,3,3-pentafluoropropane

167.3

455.0

165.4

427.4

210.3

3-chlorobenzoic acid

305.8

201.3

405.3

3-chlorophenol

285.4

3-deoxy-3-fluoro-D-glucopyranose

378.2

409.7

3-deoxy-D-glucopyranose

387.2

415.0

3-diphenylmethyl-2,4-pentanedione

387.2

382.3

3-ethyl-3-diphenylmethyl-2,4-pentanedione

388.2

370.7

3-ethylheptane

158.0

225.0

$154.6 \quad 189.4$

3-fluorotoluene

184.0

207.0

3-heptanone

236.0

210.5

3-hexanone

217.7

190.1

3-hydroxybenzoic acid

475.1

458.9

3-iodobenzoic acid

460.4

444.3

3-methyl-1,2-butadiene

159.5

150.0

3-methyl-1-butanethiol

139.6

166.7

3-methyl-1-butene

104.7

89.9 
3-methyl-1-phenyl-1H-pyrazol-5-yl dimethylcarbamate

3-methyl-2-butanethiol

$146.1 \quad 107.9$

3-methyl-3-diphenylmethyl-2,4-pentanedione $\quad 352.2 \quad 367.4$

3-methylcyclothiapentane

$192.0 \quad 238.7$

3-methylheptane

$152.6 \quad 208.9$

3-methylpentane

$110.3 \quad 165.4$

3-methylpyridine $\quad 255.0 \quad 222.6$

3-methylthiophene $\quad 204.2 \quad 206.8$

3-nitroaniline $\quad 387.2 \quad 329.7$

3-nitrophthalic anhydride $\quad 436.2 \quad 401.2$

$\begin{array}{lll}3 \text {-nitrotoluene } & 288.7 & 275.2\end{array}$

3-pentanol $\quad 204.2 \quad 217.8$

3-pentanone $\quad 234.2 \quad 164.8$

3-propyl-3-diphenylmethyl-2,4-pentanedione $\quad 349.2 \quad 373.4$

4-(1,1-dimethylethyl)-n-(1-methylpropyl)-2,6-dinitrobenzeneamine $\quad 338.8 \quad 283.9$

4-(2,4,5-trichlorophenoxy)butanoic acid $\quad 386.7 \quad 410.2$

4-(2,4-dichlorophenoxy)butyric acid $\quad 391.4 \quad 397.2$

4-(2-chlorophenylhydrazone)-3-methyl-5-isoxazolone $\quad 440.4 \quad 440.4$

4-(4-chloro-2-methylphenoxy)butanoic acid $\quad 373.4 \quad 394.3$

4-(4-nitrophenylazo)aniline $\quad 388.2 \quad 396.4$

4-(6-hexenyloxy)-3',4'difluorodiphenyldiacetylene $\quad 370.0 \quad 350.6$

4-(cis-3-hexenyloxy)-3',4'difluorodiphenyldiacetylene $\quad 364.6 \quad 332.7$

4-(cis-4-hexenyloxy)-3',4'difluorodiphenyldiacetylene $\quad 364.4 \quad 362.0$

4-(dipropylamino)-N,N-dimethyl-3,5-dinitrobenzenesulfonamide $\quad 413.6 \quad 403.7$

4-(N,N-dipropylamino)-3,5-dinitrobenzenesulphonamide $\quad 414.8 \quad 398.3$

4,4'-di-(2-methoxyethoxy)biphenyl $\quad 412.4 \quad 342.7$

4,4'dichlorodiphenylsulphone $\quad 422.0 \quad 408.8$

4,4'-didecanoyloxydiphenyldiacetylene $\quad 403.0 \quad 376.9$

4,4'-didodecanoyloxydiphenyldiacetylene $\quad 401.0 \quad 380.0$

4,4'dihydroxydiphenyl-2,2-propane $\quad 433.0 \quad 384.9$

$\begin{array}{lll}4,4^{\prime} \text {-dinitrodiphenyl ether } & 418.2 & 395.6\end{array}$

4,4'-diundecanoyloxydiphenyldiacetylene $\quad 399.0 \quad 378.6$

4,6-dichloro-N-(2-chlorophenyl)-1,3,5-triazin-2-amine $\quad 431.0 \quad 400.1$

$4[\mathrm{p}$-[bis(2-chloroethyl)amino]benzene]butanoic acid $\quad 338.9 \quad 393.0$

4'4'-diaminodiphenyl ether $\quad 365.4 \quad 398.4$

4-acetoxybenzoic acid $\quad 467.2 \quad 394.0$

4-amino-6-(1,1-dimethylethyl)-3-(methylthio)1,2,4-triazin-5(4H)-one $\quad 399.4 \quad 399.4$

4-aminoacetophenone $\quad 379.2 \quad 344.4$

4-aminobenzoic acid $\quad 461.4 \quad 514.1$

4-amino-N-(6-methoxy-3-pyridazinyl)benzenesulfonamide $\quad 453.4 \quad 466.9$

4-aminopyridine $\quad 429.9 \quad 313.0$

4-benzoyl-1-naphthol $\quad 440.6 \quad 435.8$

4-bromo-2,5-dichloroaniline $\quad 343.4 \quad 338.6$

4-bromo-2,5-dichlorophenol $\quad 343.4 \quad 342.5$

4-bromophenol $\quad 336.0 \quad 344.8$

4-chloroazobenzene $\quad 361.2 \quad 380.2$

4-chlorobenzoic acid $\quad 312.9 \quad 457.9$

4-chlorobiphenyl $\quad 348.6 \quad 356.0$

4-chlorobut-2-ynyl 3-chlorophenylcarbamate $\quad 344.1 \quad 320.1$

4-chlorophenol $\quad 315.9 \quad 322.5$

4-chlorophenoxyacetic acid $\quad 429.6 \quad 380.8$

4-chlorophenyl 4-chlorobenzenesulfonate $\quad 360.0 \quad 355.1$

4-chlorophenylbenzenesulfonate $\quad 332.2 \quad 337.1$ 
4-ethoxy-4'fluorodiphenyldiacetylene

364.1

4-ethoxy-4'-trifluoromethyldiphenyldiacetylene

400.2

424.9

394.4

4-ethoxybenzoic acid

472.8

402.1

4-ethoxyisonitrosoacetanilide

490.0

447.5

4-ethoxyphenylacetic acid

360.2

4-ethylbenzoic acid

386.2

4-ethynyl-1-[(4-ethynylphenyl)methoxy]benzene

371.2

358.8

4-fluorotoluene

216.5

359.8

4-heptanone

240.2

4-hexylresorcinol

341.5

4-hydroxy-3,5-diiodobenzonitrile

482.9

4-hydroxyazobenzene

425.2

4-hydroxybenzoic acid

488.1

4-hydroxyphenylacetic acid

423.6

4-hydroxyphenylpropionic acid

402.5

402.8

233.9

210.5

362.4

518.8

440.8

518.5

397.2

4-iodobenzoic acid

543.8

384.4

4-methoxybenzoic acid

4-methoxyphenol

4-methoxyphenylacetic acid

457.8

328.4

358.1

4-methoxyphenylbutyric acid

330.9

4-methoxyphenylpropionic acid

376.9

4-methyl-7-aminocoumarin

499.9

4-methyl-7-diethylaminocoumarin

343.8

4-methyl-7-dimethylaminocoumarin

416.1

4-methyl-7-hydroxycoumarin

460.7

4-methylcyclohex-1-ene

153.6

4-methylheptane

152.2

4-methylpent-1-ene

118.9

4-methylphenanthrene

324.9

4-methylsulphonyl-2,6-dinitro-N,N-dipropylaniline

424.3

4-methylthiazole

4-methylthio-3,5-xylyl methylcarbamate

4-n-butoxy-2',3',4'-trifluorodiphenylacetylene

229.1

393.8

344.4

4-n-butoxy-4'-fluorodiphenylacetylene

346.7

4-n-butoxy-4'-trifluoromethyldiphenyldiacetylene

414.3

4-n-butoxybenzoic acid

4-n-butyl-3',4'-difluorodiphenylacetylene

432.2

323.5

340.8

4-n-butyl-3',4'-difluorodiphenyldiacetylene

329.9

356.8

502.1

402.4

318.5

281.3

352.0

347.1

442.9

354.0

453.3

446.8

181.2

208.9

94.7

347.7

413.6

222.9

364.7

355.0

354.4

332.4

401.6

316.2

334.1

315.9

4-n-ethoxy-2',3',4'-trifluorodiphenylacetylene

343.4

344.8

4-n-ethoxy-2',4'-difluorodiphenylacetylene

354.4

344.5

4-n-ethoxy-4'-fluorodiphenylacetylene

301.2

4-n-ethyl-3',4'-difluorodiphenylacetylene

314.9

380.0

322.0

320.9

344.1

294.7

4-n-hexylbenzoic acid

345.2

4-n-hexyloxy-2',3',4'-trifluorodiphenylacetylene

373.3

4-n-hexyloxy-2',4'-difluorodiphenylacetylene

361.9

4-n-hexyloxy-3',4'-difluorodiphenylacetylene

323.6

361.7

4-n-hexyloxy-4'-trifluoromethyldiphenyldiacetylene

394.8

402.0

361.7

4-nitro-4'-methylbenzylidene aniline

401.0

342.2

4-nitro-5-methylphenol

420.2

4-nitrophthalic anhydride

388.2

283.5

349.4

372.5

401.2 
4-nitrotoluene

4-n-octyloxy-N-(3,5-dimethoxybenzylidene)aniline

4-n-octyloxy-N-(3,5-dimethylbenzylidene)aniline

4-n-octyloxy-N-(4-methoxybenzylidene)aniline

4-n-pentoxy-2',3',4'-trifluorodiphenylacetylene

4-n-pentoxy-4'-fluorodiphenylacetylene

4-n-pentoxybenzoic acid

4-n-pentyl-3',4'-difluorodiphenylacetylene

4-n-pentyl-3',4'-difluorodiphenyldiacetylene

4-n-pentyl-4'-fluorodiphenylacetylene

4-n-pentylbenzoic acid

4-n-propoxy-2',3',4'-trifluorodiphenylacetylene

4-n-propoxy-2',4'-difluorodiphenylacetylene

4-n-propoxy-4'-fluorodiphenylacetylene

4-n-propoxybenzoic acid

4-n-propyl-3',4'-difluorodiphenylacetylene

4-n-propyl-3',4'difluorodiphenyldiacetylene

4-n-propyl-4'-fluorodiphenylacetylene

4-octadecynoic acid

4-oxaheptane

4-propoxy-4'-trifluoromethyldiphenyldiacetylene

4-propylbenzoic acid

4'-propylbiphenyl-4-carbonitrile

4-tert-butylbenzoic acid

4-tert-butylphenol

4-trans-(3-fluoro-4-cyanophenyl)cyclohexyl (E)-but-2-enoate

4-trans-(4-bromophenyl)cyclohexyl (E)-2-butenoate

4-trans-(4-chlorophenyl)cyclohexyl (E)-2-butenoate

4-trans-(4-fluorophenyl)cyclohexyl (E)-2-butenoate

4-trans-(4-fluorophenylethyl)cyclohexyl (E)-butenoate

4-trans-(trifluoromethoxyphenyl)cyclohexyl (E)-but-2-enoate

4-trans-cyanocyclohexyl (E) 2-butenoate

5 '-(trifluoromethanesulphonamide)acet-2',4-xylidide

5,5-bis(3,3-dimethylbutyl)2,2,8,8-tetramethylnonane

5,5-dimethylperhydro-1,3-oxazine-2-one

$5,6,7,8$-tetrahydroquinoline

5,6-dibutyl-5,6-bis(4-tert-butylphenyl)decane

5-[2-chloro-4-(trifluoromethyl)phenoxy]-2-nitrobenzoic acid

5-amino-4-chloro-2-phenyl-3(2H)-pyridazinone

5-bromo-6-methyl-3-(1-methylpropyl)-2,4(1H,3H)-pyrimidinedione

5-butyl-2-ethylamino-6-methylpyrimidin-4-ol

5-chloro-3-(1,1-dimethylethyl)-6-methyl-2,4(1H,3H)-pyrimidinedione

5 -isopropyl-m-tolyl methylcarbamate

5-methyl N-(methylcarbamoyloxy)thioacetimidate

5-methyl-1,2,4-triazolo[3,4-b]benzothiazole

5-methylnonane

5-methyltetrazole

5-methylthiazole

5-nonanone

5 -octadecynoic acid

6-(4-biphenyl)-1-hexene

6,8,9-trimethyladenine

6,9-dimethyl-8-butyladenine
324.8

316.3

324.7

377.3

315.8

330.9

422.2

323.1

355.1

337.4

395.0

327.3

326.9

356.8

426.7

311.0

343.7

324.0

348.0

158.4

315.9

422.0

338.8

440.0

373.2

393.2

388.2

386.2

354.2

335.2

340.2

366.2

457.3

472.7

440.1

222.7

386.0

436.6

479.2

428.3

432.5

448.0

361.3

352.7

460.2

186.7

418.0

232.8

223.2

325.0

274.5

438.0

409.2
311.0

357.4

349.6

346.9

358.7

358.2

401.4

323.9

340.2

323.6

370.8

350.4

350.1

349.8

401.8

306.7

326.6

306.3

307.9

212.3

326.2

364.3

358.5

450.0

330.1

364.5

339.9

328.6

317.0

320.6

342.2

318.8

487.0

347.2

440.1

293.3

380.6

399.1

479.2

411.1

399.9

469.0

330.8

351.9

343.5

238.7

434.0

222.9

241.3

307.9

289.2

450.1

404.6 
6-deoxy-6-fluoro-D-glucopyranose

412.2

408.9

6-deoxy-D-glucopyranose

409.2

364.3

6-methyl-1,3-dithiolo[4,5-b]quinoxalin-2-one

443.2

443.2

6-octadecynoic acid

324.0

307.9

7,8-benzoquinoline

324.1

347.9

7-octadecynoic acid

322.0

307.9

8-(4-biphenyl)-1-octene

306.5

8-[4-(4'-n-butylbiphenyl)]-1-octene

291.5

310.9

8-octadecynoic acid

315.6

307.9
414.5

9-fluorenone

320.0

9-heptadecanone

356.4

296.4

9H-pyrido[3,4-b]indole

323.9

449.9

9-methylfluorene

471.5

225.8

9-octadecynoic acid

319.2

307.9

a-(trifluoromethoxy)-a,a-difluoromethyl acetate

319.0

229.8

a,a'-dibromo-m-xylene

167.4

297.5

a, a'-dibromo-o-xylene

350.2

a,a'-dichloro-m-xylene

368.2

297.5

a,a'-dichloro-o-xylene

307.2

270.7

a,a'-dichloro-p-xylene

328.2

270.7

a-alanyl-a-alanine (DL)

373.2

270.7

acebutolol

483.2

458.5

acenaphthene

394.2

420.4

acenaphthylene

366.6

336.7

acetaldehyde

362.0

300.7

acetamide

242.9

175.7

acetanilide

353.0

323.3

acetohexamide

387.5

374.0

acetone

461.2

398.1

acetonitile

acetylacetone enol

176.6

199.6

229.3

186.7

acetylene

254.8

245.8

$192.4 \quad 200.8$

$334.3 \quad 295.3$

roacetic acid

334.4

295.3

acridine

383.2

393.1

acrylamide

358.0

337.3

acrylic acid

285.5

364.3

acrylonitrile

189.6

211.0

a-D-glucopyranose (a-D-glucose)

423.2

453.0

a-D-glucose

414.0

453.0

adipic acid

426.4

376.1

alanylglycine (with decomp)

508.0

453.8

Albendazole

482.2

460.9

Aldicarb

372.2

353.7

Aldoxycarb

414.2

454.7

allobarbitiol

442.6

389.0

a-methylacrylic acid

287.5

301.8

a-methylstyrene

250.8

200.5

Ametryn

358.2

379.3

Aminocarb

367.2

344.6

amobarbital

429.2

418.5

a-naphthol

369.0

335.5

a-naphthyl acetate

319.2

394.1 


\begin{tabular}{|c|c|c|}
\hline androstanolone & 454.0 & 441.7 \\
\hline Anilazine & 433.2 & 464.0 \\
\hline aniline & 267.1 & 294.9 \\
\hline anisaldazine & 442.0 & 442.0 \\
\hline anthracene & 488.9 & 431.0 \\
\hline anthraquinone & 558.0 & 604.4 \\
\hline aprobarbital & 414.2 & 385.3 \\
\hline atenolol & 420.2 & 418.5 \\
\hline Atrazine & 448.2 & 399.1 \\
\hline azelaic acid & 380.0 & 381.2 \\
\hline Azimsulfuron & 443.2 & 523.7 \\
\hline azoxybenzene & 309.2 & 309.2 \\
\hline azulene & 373.5 & 295.2 \\
\hline b(4-chlorophenoxy)-a-(1,1-dimethylethyl)-1H-1,2,4-triazole-1-ethanol & 377.8 & 413.5 \\
\hline b-alanyl-b-alanine & 480.1 & 458.5 \\
\hline barbital & 462.6 & 500.8 \\
\hline beclomethasone-17,21-dipropionate & 393.2 & 421.0 \\
\hline Bendiocarb & 403.2 & 443.6 \\
\hline bendroflumethiazide & 495.2 & 520.8 \\
\hline Benfluralin & 339.2 & 365.1 \\
\hline benzaldehyde & 216.0 & 293.0 \\
\hline benzamide & 402.3 & 399.6 \\
\hline benzanilide & 436.5 & 431.4 \\
\hline benzene & 278.7 & 324.4 \\
\hline benzene-hexa-n-hexanoate & 368.7 & 351.0 \\
\hline benzidine & 400.2 & 565.0 \\
\hline benzil & 368.0 & 420.3 \\
\hline benzimidazole & 443.2 & 428.6 \\
\hline benzo[c]cinnoline & 432.2 & 366.9 \\
\hline benzofluoranthene & 424.0 & 348.9 \\
\hline benzoic acid & 395.5 & 434.7 \\
\hline benzoic anhydride & 313.2 & 265.6 \\
\hline benzonitrile & 260.3 & 308.9 \\
\hline benzophenone & 324.2 & 328.8 \\
\hline benzothiazole & 275.6 & 277.6 \\
\hline benzothiophene & 304.5 & 261.6 \\
\hline benzotrichloride & 236.0 & 288.6 \\
\hline benzotrifluoride & 244.0 & 179.4 \\
\hline benzoxazole & 302.5 & 247.5 \\
\hline benzthiazide & 508.2 & 508.2 \\
\hline benzyl alcohol & 257.6 & 268.1 \\
\hline benzyl benzoate & 293.1 & 344.6 \\
\hline benzylbromide & 271.8 & 258.0 \\
\hline benzyliodide & 299.5 & 293.6 \\
\hline benzylmethylsulfone & 400.5 & 381.4 \\
\hline betamethasone & 503.0 & 520.9 \\
\hline betamethasone-17-valerate & 456.0 & 431.6 \\
\hline beta-sitosterol & 413.3 & 358.6 \\
\hline betaxolol & 344.2 & 346.9 \\
\hline biphenyl & 341.5 & 382.5 \\
\hline bis(2-cyanoethyl)-N-nitroamine & 327.0 & 382.0 \\
\hline bis-(4-aminophenyl)methane & 363.7 & 405.8 \\
\hline bis(4-chlorophenyl)acetic acid & 440.2 & 450.3 \\
\hline
\end{tabular}


bis-[3,5-di-tert-butyl-4-hydroxybenzyl]sulfide

carvedilol

Chlorimuron

chlorobenzene

chlorothiazide

chlorotoluron 


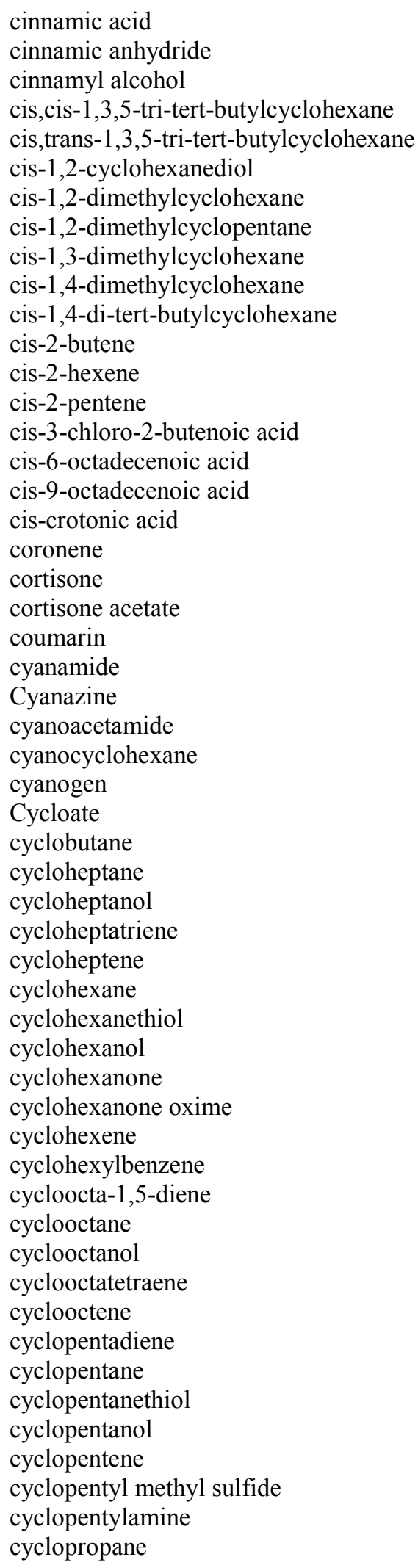




\begin{tabular}{|c|c|c|}
\hline cyclopropylamine & 237.8 & 265.2 \\
\hline Cyprazine & 440.2 & 440.2 \\
\hline D mannitol & 439.1 & 403.5 \\
\hline D sorbitol & 366.5 & 403.5 \\
\hline Dacthal & 428.2 & 356.1 \\
\hline Daimuron & 476.2 & 481.1 \\
\hline decachlorobiphenyl & 577.7 & 649.8 \\
\hline decacyclene & 666.0 & 635.4 \\
\hline decanal & 268.2 & 285.2 \\
\hline decanoic acid & 304.5 & 326.6 \\
\hline decyl methacrylate & 250.7 & 290.1 \\
\hline deoxycorticosterone & 414.0 & 475.7 \\
\hline deoxycorticosterone acetate & 430.0 & 402.6 \\
\hline Desmetryn & 358.2 & 340.4 \\
\hline desoxycorticosterone pivalate & 476.2 & 476.2 \\
\hline dexamethasone & 539.0 & 520.9 \\
\hline Diallate & 298.2 & 239.3 \\
\hline diaminoethane & 284.2 & 247.2 \\
\hline dibenzo[c,e][1,2]dithiin & 386.2 & 411.6 \\
\hline dibenzodioxin & 395.7 & 435.9 \\
\hline dibenzofuran & 355.7 & 341.2 \\
\hline dibenzothiophene & 371.0 & 375.3 \\
\hline dibromodichloromethane & 294.4 & 242.6 \\
\hline dibromodifluoroethylene & 162.8 & 195.2 \\
\hline Dibutyl phthalate & 238.2 & 336.6 \\
\hline dichloroacetic acid & 286.5 & 345.3 \\
\hline dichloromethane & 178.2 & 192.7 \\
\hline dicyanomethane & 305.0 & 285.5 \\
\hline dienestrol & 500.2 & 435.8 \\
\hline diethyl disulfide & 171.6 & 208.5 \\
\hline diethyl ether & 156.9 & 169.7 \\
\hline diethyl o-phthalate & 269.9 & 314.3 \\
\hline Diethyl phthalate & 270.2 & 314.3 \\
\hline diethyl sulfide & 169.2 & 188.9 \\
\hline diethyl terephthalate & 317.2 & 314.3 \\
\hline diethylstilbestrol & 442.0 & 382.3 \\
\hline Difenoxuron & 412.2 & 422.8 \\
\hline diflubenzuron & 512.2 & 452.6 \\
\hline difluoromethane & 136.4 & 119.9 \\
\hline dihydroxyethane & 260.6 & 284.4 \\
\hline diiodomethane & 279.2 & 302.0 \\
\hline diisopropyl ketone & 204.8 & 175.9 \\
\hline diisopropyl sulfide & 195.1 & 137.7 \\
\hline Dimepiperate & 312.2 & 339.5 \\
\hline Dimetan & 318.7 & 324.1 \\
\hline Dimethametryn & 338.2 & 331.7 \\
\hline dimethoxymethane & 168.0 & 148.4 \\
\hline dimethyl (2,2,2-trichloro-1-hydroxyethyl)phosphonate & 384.0 & 384.0 \\
\hline dimethyl amine & 181.0 & 164.7 \\
\hline dimethyl ether & 131.7 & 101.8 \\
\hline dimethyl fumarate & 375.0 & 290.5 \\
\hline dimethyl maleate & 254.0 & 290.5 \\
\hline dimethyl oxalate & 327.6 & 455.9 \\
\hline
\end{tabular}




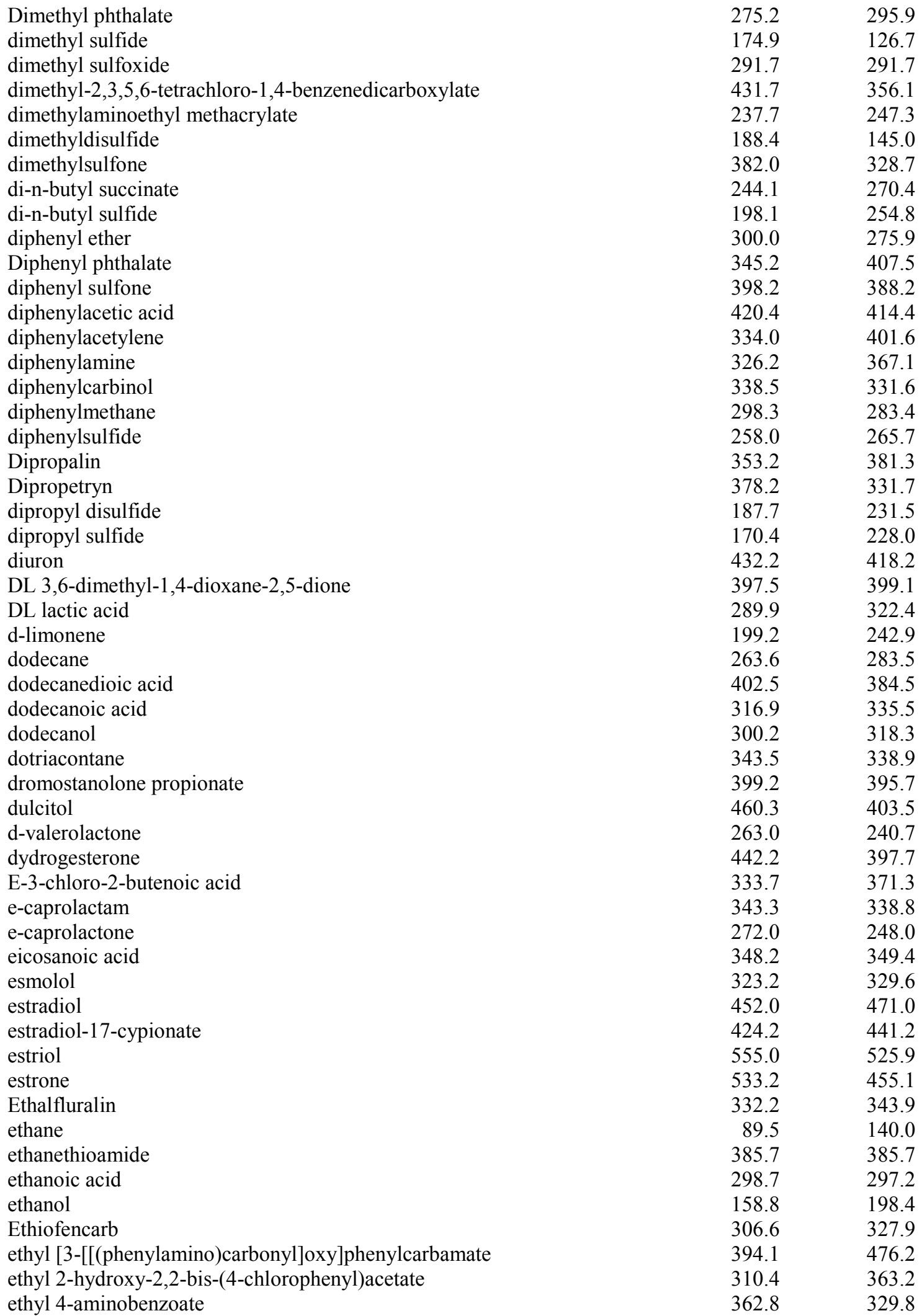




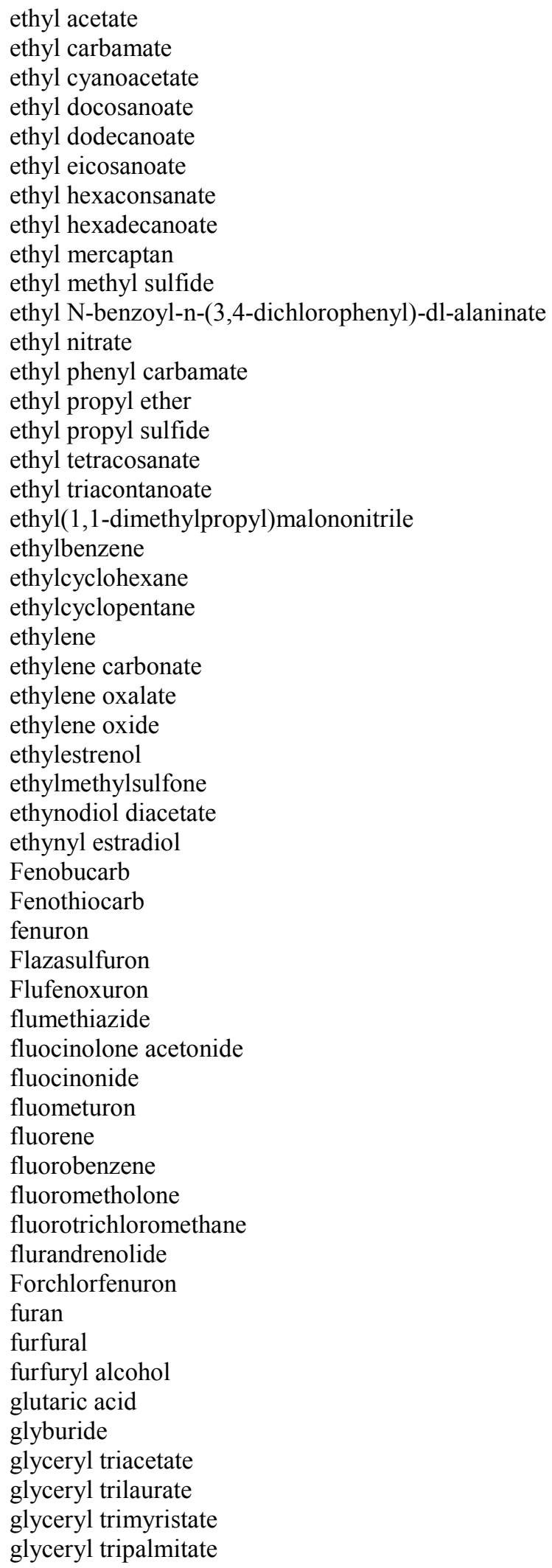




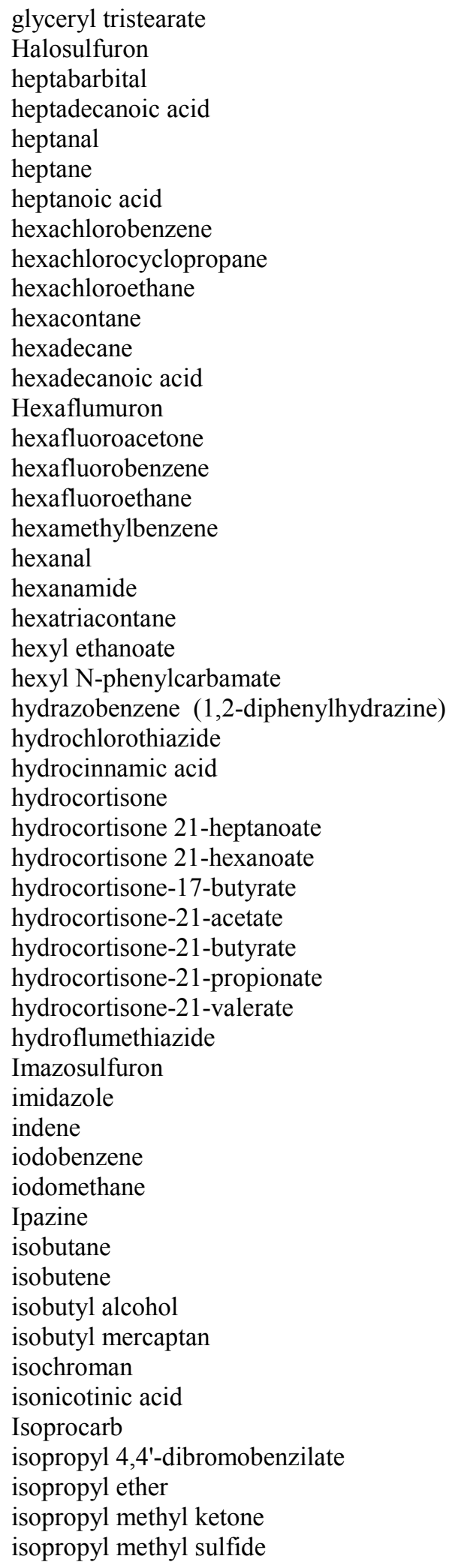

345.7

449.2

447.2

334.3

229.3

182.6

265.8

505.0

376.0

458.0

373.2

291.1

335.7

476.7

147.7

278.3

173.1

438.7

243.2

374.0

349.2

212.1

328.0

407.2

547.2

321.2

485.0

384.2

388.2

395.0

497.0

463.2

484.2

457.2

543.7

456.2

361.9

271.7

241.8

206.8

360.2

113.7

132.4

171.2

128.3

277.5

593.0

367.2

348.1

187.8

180.0

171.7
360.7

419.5

472.0

342.5

254.7

229.7

307.2

534.5

406.1

461.3

365.1

292.2

339.8

451.7

122.5

328.9

210.6

486.1

240.6

338.0

344.8

262.5

359.3

402.9

592.2

327.2

571.6

444.2

447.7

468.8

505.7

456.7

462.6

451.8

560.4

502.7

308.7

242.2

282.8

204.1

340.6

117.7

139.7

195.4

136.8

314.0

452.8

319.8

367.5

118.5

182.3

132.9 


\begin{tabular}{|c|c|c|}
\hline isopropyl nitrate & 190.9 & 209.9 \\
\hline isopropyl phenylcarbamate & 359.5 & 313.5 \\
\hline isopropyl-3-chlorophenylcarbamate & 313.9 & 327.8 \\
\hline isopropylbenzene & 177.1 & 210.1 \\
\hline Isoproturon & 431.2 & 371.1 \\
\hline isoquinoline & 299.6 & 277.3 \\
\hline Isouron & 392.7 & 414.0 \\
\hline L-carvone & 247.7 & 335.7 \\
\hline levulinic acid & 306.2 & 304.1 \\
\hline L-iditol & 352.8 & 403.5 \\
\hline linoelaidic acid & 303.0 & 295.6 \\
\hline linuron & 367.2 & 365.0 \\
\hline Lufenuron & 439.2 & 469.1 \\
\hline lynestrenol & 432.2 & 358.1 \\
\hline m-(methylamino)benzoic acid & 402.2 & 428.9 \\
\hline m-acetamidobenzoic acid & 523.2 & 510.3 \\
\hline m-acetotoluidide & 339.2 & 387.8 \\
\hline m-acetoxybenzoic acid & 404.2 & 419.3 \\
\hline maleic anhydride & 325.7 & 293.5 \\
\hline malonamide & 443.0 & 486.9 \\
\hline m-alpha-bromotolunitrile & 366.2 & 335.0 \\
\hline m-aminoacetanilide & 361.2 & 435.3 \\
\hline m-aminobenzaldehyde & 303.2 & 329.6 \\
\hline m-aminobenzamide & 384.2 & 469.9 \\
\hline m-aminobenzyl alcohol & 370.2 & 333.5 \\
\hline m-aminophenol & 399.0 & 335.1 \\
\hline m-benzenedicarboxylic acid & 621.2 & 653.9 \\
\hline m-bromoacetanilide & 361.2 & 409.2 \\
\hline m-bromoacetophenone & 281.2 & 314.5 \\
\hline m-bromoaniline & 291.2 & 301.3 \\
\hline m-bromobenzamide & 428.2 & 439.9 \\
\hline m-bromobenzoic acid & 428.2 & 425.0 \\
\hline m-bromobenzonitrile & 311.2 & 313.7 \\
\hline m-bromobenzyl bromide & 314.2 & 295.5 \\
\hline m-bromomethylbenzoic acid & 429.2 & 438.7 \\
\hline m-bromophenol & 306.2 & 305.2 \\
\hline m-bromophenyl urea & 438.2 & 464.8 \\
\hline m-bromotoluene & 233.2 & 246.8 \\
\hline m-chloroacetanilide & 352.2 & 392.0 \\
\hline m-chloroaniline & 263.2 & 281.5 \\
\hline m-chlorobenzaldehyde & 291.2 & 279.9 \\
\hline m-chlorobenzamide & 407.2 & 420.2 \\
\hline m-chlorobenzonitrile & 314.2 & 293.9 \\
\hline m-chlorobenzyl bromide & 295.2 & 277.2 \\
\hline m-chloroethylbenzene & 210.6 & 223.0 \\
\hline m-chlorotoluene & 225.2 & 227.1 \\
\hline m-cyanoaniline & 326.2 & 343.6 \\
\hline m-cyanobenzaldehyde & 351.2 & 342.0 \\
\hline m-cyanobenzoic acid & 490.2 & 467.4 \\
\hline m-cymene & 209.5 & 224.8 \\
\hline m-diacetylbenzene & 305.2 & 333.0 \\
\hline m-diethylbenzene & 189.3 & 202.2 \\
\hline
\end{tabular}




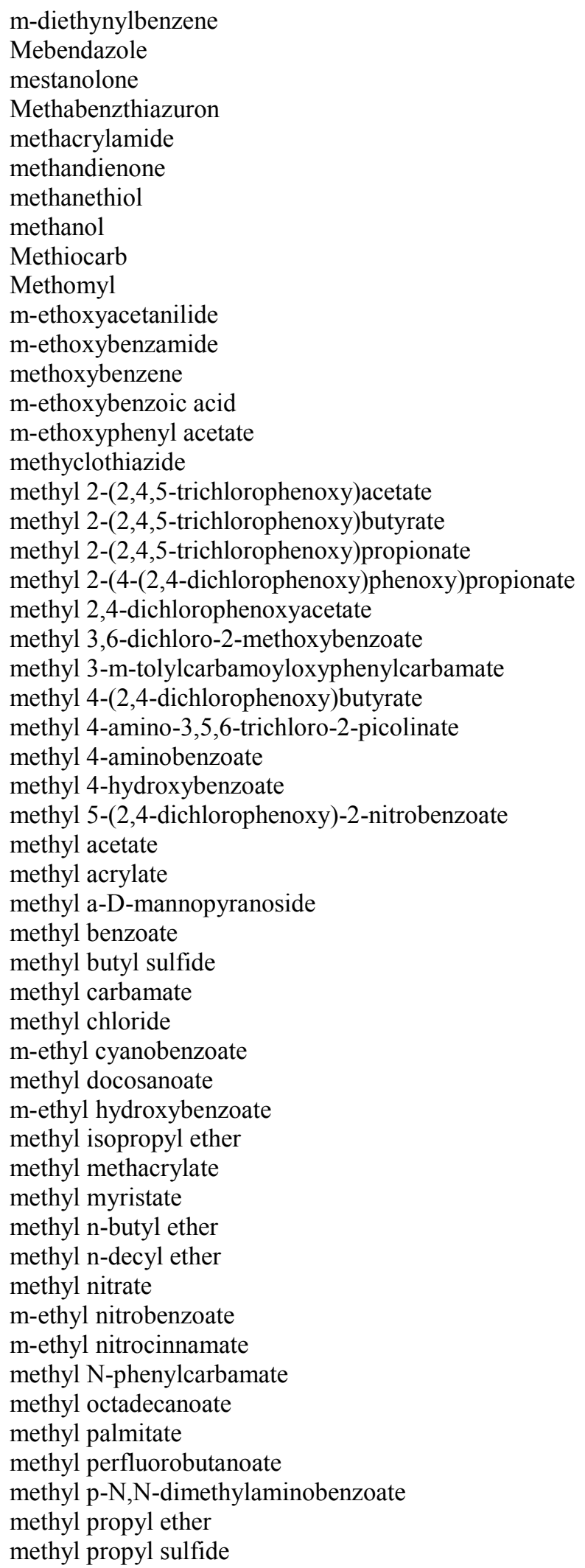

276.2

561.7

465.2

393.2

385.1

436.2

150.2

175.3

393.2

351.2

369.2

412.2

293.2

410.2

307.2

498.2

361.9

316.5

360.6

314.4

315.4

304.6

423.8

309.6

394.3

385.1

398.5

358.3

174.9

197.5

455.2

261.0

175.6

328.6

174.5

329.2

325.0

346.2

127.3

225.0

291.6

157.5

243.5

190.2

320.2

352.2

325.0

310.9

307.2

191.4

371.8

134.0

160.2
334.7

495.8

421.9

491.4

316.7

369.2

88.2

225.6

364.7

351.9

391.8

413.6

249.4

426.4

293.4

518.6

313.4

325.6

318.1

354.8

299.1

321.3

493.3

316.2

382.0

320.8

324.2

369.9

168.3

205.3

388.0

259.6

210.4

319.9

181.6

383.0

320.4

332.8

111.2

187.4

288.4

193.2

271.7

184.1

328.6

368.9

329.3

307.1

298.6

224.9

272.4

169.7

188.9 
methyl tert-butyl ether

164.6

196.1

methyl tert-butyl sulfide

190.8

220.9

methyl tetrachloroterephthalic acid ester

444.3

427.7

methyl-3,4-dichlorophenylcarbamate

381.4

361.1

methylamine

179.7

191.1

m-ethylbenzoic acid

320.2

384.5

methylcyclobutane

138.6

151.0

methylcyclohexane

146.6

184.7

methylcyclopentane

130.7

169.8

Methyldymron

345.2

452.3

methylenecyclobutane

138.5

158.4

methylhydrazine

220.8

214.4

m-ethylphenol

269.2

272.9

methylphenylsulfide

256.4

241.0

methylphosphonyl chlorofluoride

250.7

250.7

methylphosphonyl dichloride

306.1

306.1

methylphosphonyl difluoride

236.3

236.3

methylprednisolone

513.2

methylprednisolone-21-acetate

498.2

methyltestosterone acetate

448.0

529.8

471.1

methyltestosterone propionate

418.0

429.8

m-ethyltoluene

177.2

388.0

metobromuron

369.2

Metolcarb

349.7

metoprolol

308.2

Metoxuron

399.7

m-fluorobenzoic acid

397.2

m-hydroxyacetanilide

422.2

m-hydroxyacetophenone

369.2

m-hydroxybenzaldehyde

381.2

m-hydroxybenzaldehyde oxime

363.2

440.2

m-hydroxybenzamide

346.2

m-hydroxybenzyl alcohol

285.4
392.2

m-hydroxytoluene

m-iodoacetanilide

298.2

m-iodoaniline

m-iodobenzaldehyde

330.2

$\mathrm{m}$-iodobenzaldehyde oxime

335.2

m-iodobenzamide

459.2

m-iodobenzyl bromide

324.2

m-iodophenol

313.2

m-iodophenyl acetate

m-iodotoluene

m-isopropylbenzoic acid

311.2

246.0

324.2

$\mathrm{m}$-methoxyacetanilide

353.2

m-methoxyacetophenone

368.2

m-methoxyaniline

261.2

313.2

383.2

m-methoxybenzoic acid

312.2

311.2

305.2

218.6

364.4

341.5

318.5

414.6

385.1

438.7

348.3

333.5

380.7

473.8

337.1

280.7

426.0

320.6

318.9

367.2

459.2

313.5

324.5

302.2

266.2

390.8

390.9

306.1

314.8

337.1

430.1

281.3

320.8

m-methyl aminobenzoate

294.7

m-methyl chlorobenzoate

294.2

277.5 


\begin{tabular}{|c|c|c|}
\hline m-methyl cyanobenzoate & 338.2 & 331.6 \\
\hline m-methyl hydroxybenzoate & 343.2 & 324.2 \\
\hline m-methyl iodobenzoate & 327.2 & 311.6 \\
\hline m-methyl nitrobenzoate & 352.2 & 319.5 \\
\hline m-methyl nitrocinnamate & 397.2 & 365.4 \\
\hline m-N,N'-diacetylphenylenediamide & 464.2 & 444.5 \\
\hline m-nitroacetanilide & 428.2 & 433.9 \\
\hline m-nitroacetophenone & 354.2 & 342.8 \\
\hline m-nitrobenzaldehyde & 331.2 & 328.0 \\
\hline m-nitrobenzaldoxime & 395.2 & 375.6 \\
\hline m-nitrobenzamide & 415.2 & 468.3 \\
\hline m-nitrobenzoic acid & 414.3 & 453.4 \\
\hline m-nitrobenzonitrile & 391.2 & 342.0 \\
\hline m-nitrobenzoyl chloride & 308.2 & 319.4 \\
\hline m-nitrobenzyl alcohol & 300.2 & 332.0 \\
\hline m-nitrobenzyl bromide & 332.2 & 322.0 \\
\hline m-nitrobenzyl chloride & 319.2 & 306.7 \\
\hline m-nitrobenzyl cyanide & 335.2 & 348.4 \\
\hline m-nitrobenzyl iodide & 359.2 & 357.6 \\
\hline m-nitrobromobenzene & 329.2 & 299.7 \\
\hline m-nitroiodobenzene & 311.2 & 319.0 \\
\hline m-nitrophenentole & 307.2 & 323.8 \\
\hline m-nitrophenol & 371.2 & 333.6 \\
\hline m-nitrophenyl acetate & 329.2 & 310.1 \\
\hline m-nitrophenylacetic acid & 393.2 & 392.5 \\
\hline m-N-methylnitroaniline & 341.2 & 343.0 \\
\hline m-n-propylbenzoic acid & 316.2 & 386.3 \\
\hline mono(2,2-dimethylhydrazide) butanedioic acid & 431.4 & 418.0 \\
\hline Monuron & 443.7 & 402.3 \\
\hline m-phenylenediacetic acid & 443.2 & 418.5 \\
\hline m-phenylenediamine & 335.5 & 374.3 \\
\hline m-propylphenol & 299.2 & 288.2 \\
\hline m-terphenyl & 360.0 & 450.1 \\
\hline m-tolualdehyde oxime & 333.2 & 326.4 \\
\hline m-toluamide & 368.2 & 415.4 \\
\hline m-toluic acid & 381.9 & 400.5 \\
\hline m-toluidine & 241.7 & 276.8 \\
\hline m-tolunitrile & 250.2 & 289.2 \\
\hline m-tolylurea & 415.2 & 443.5 \\
\hline m-xylene & 225.3 & 251.3 \\
\hline myo-inositol & 496.9 & 421.8 \\
\hline N-(1-ethylpropyl)-2,6-dinitro-3,4-xylidine & 327.5 & 297.3 \\
\hline N-(2-chloroethyl)-2,6-dinitro-N-propyl-4-(trifluoromethyl)benzeneamine & 318.4 & 390.2 \\
\hline N(3),N(3)-diethyl-2,4-dinitro-6-(trifluoromethyl)-1,3-benzenediamine & 372.1 & 390.1 \\
\hline N-(3,4-dichlorophenyl)-2-methyl-2-propenamide & 395.5 & 371.2 \\
\hline N'-(3-chloro-4-methoxyphenyl)-N,N-dimethylurea & 399.2 & 414.6 \\
\hline $\mathrm{N}$-(3-chloro-4-methylphenyl)-2-methylpentanamide & 353.2 & 378.9 \\
\hline $\mathrm{N}$-(4-chlorophenyl)-2,2-dimethylpentanamide & 360.2 & 388.1 \\
\hline N'-(4-chlorophenyl)-N-methoxy-N-methylurea & 353.4 & 350.7 \\
\hline $\mathrm{N}$-(cyclopropylmethyl)-2,6-dinitro-n-propyl-4-(trifluoromethyl)benzenamine & 305.8 & 297.5 \\
\hline N,N'-(2-hydroxyethyl)-1,4-diaminoanthraquinone & 521.2 & 533.1 \\
\hline N,N-(2-hydroxyethyl)-4-(4-nitrophenyl)azoaniline & 484.2 & 431.3 \\
\hline N,N-(2-hydroxyethyl)-4-phenylazoaniline & 407.0 & 428.4 \\
\hline
\end{tabular}


N,N-diethyl-2-(1-naphthyloxy)propionamide

345.3

351.2

$\mathrm{N}, \mathrm{N}$-dimethyl-1,3-propanediamine

$194.4 \quad 246.3$

$\mathrm{N}, \mathrm{N}$-dimethyl-2,2-diphenylacetamide

$407.1 \quad 322.7$

$\mathrm{N}, \mathrm{N}$-dimethyl-2,2-diphenylbenzeneacetamide

$402.0 \quad 390.0$

N,N-dimethyl-2-methylcarbamoyloxyimino-2-(methylthio)acetamide $\quad 372.2 \quad 373.2$

N,N-dimethyl-4-phenylazoaniline $\quad 389.2 \quad 323.1$

N,N-dimethylaniline $\quad 275.6 \quad 228.7$

$\begin{array}{lll}\mathrm{N}, \mathrm{N} \text {-dimethylformamide } & 212.9 & 212.9\end{array}$

N,N'-dimethylhydrazine $\quad 264.3 \quad 269.2$

N,N-dimethylhydrazine $\quad 216.0 \quad 216.0$

N,N'-dimethyl-N,N'dinitro-1,2-ethanediamine $\quad 410.0 \quad 355.3$

N,N'-dimethyl-N,N'dinitro-1,6-hexanediamine $\quad 331.0 \quad 368.1$

N,N-dimethyl-N'-[3-(trifluoromethyl)-phenyl]urea $\quad 434.1 \quad 426.4$

N,N-dimethyl-N'-[4-(1-methylethyl)phenyl]urea $\quad 430.5 \quad 371.1$

N,N'-di-n-hexyladipamide $\quad 432.0 \quad 388.9$

N,N'-dinitro-diaminomethane $\quad 371.0 \quad 413.4$

N,N'-dinitroethanediamine $\quad 450.0 \quad 412.0$

N,N'-di-n-propyladipamide $\quad 452.0 \quad 385.1$

$\mathrm{N}$-[(1,1,2,2-tetrachloroethyl)thio]-4-cyclohexene-1,2-dicarboximide $\quad 432.0 \quad 430.6$

$\mathrm{N}-[4-($ methoxymethyl)-1-[2-(2-thienyl)ethyl]-4-piperidinyl]-N-phenyl-
propanamide

N-[4-methyl-3-[[(trifluoromethyl)sulfonyl]amino]phenyl]acetamide $\quad 455.7 \quad 474.8$

N-[5-(1,1-dimethylethyl)-1,3,4-thiadiazol-2-yl]-N,N'-dimethylurea 435.3

$\begin{array}{lll}\text { N-acetyl-D-leucine amide } & 404.0 & 427.3\end{array}$

$\begin{array}{lll}\mathrm{N} \text {-acetylglycine amide } & 408.2 & 454.0\end{array}$

$\begin{array}{lll}\mathrm{N} \text {-acetylglycyl-L-prolinamide } & 457.8 & 478.4\end{array}$

$\begin{array}{lll}\mathrm{N} \text {-acetyl-L-alanine amide } & 431.0 & 456.2\end{array}$

$\begin{array}{lll}\mathrm{N} \text {-acetyl-L-isoleucinamide } & 529.6 & 427.3\end{array}$

N-acetyl-L-prolyl-glycinamide $\quad 434.1 \quad 478.4$

$\begin{array}{lll}\mathrm{N} \text {-acetyl-pyrazinamide } & 366.7 & 366.7\end{array}$

N-acetylsarcosinamide $\quad 412.7 \quad 363.3$

$\begin{array}{lll}\mathrm{N}-\text { allyl-N-phenylthiourea } & 375.0 & 375.0\end{array}$

nandrolone $\quad 385.2 \quad 395.1$

nandrolone decanoate $\quad 308.2 \quad 382.6$

$\begin{array}{lll}\text { naphthalene } & 353.4 & 339.3\end{array}$

naphthalene 1,8-disulfide $\quad 394.8 \quad 339.8$

naphthalene 1,8-disulfide s-oxide $\quad 421.2 \quad 421.2$

$\begin{array}{lll}\mathrm{N} \text {-benzylaniline } & 305.6 & 345.9\end{array}$

$\begin{array}{lll}\text { N-butyl dodecanamide } & 322.1 & 340.0\end{array}$

n-butyl mercaptan $\quad 157.5 \quad 192.3$

N-butyl tetradecanamide $\quad 336.1 \quad 345.3$

n-butylbenzene $\quad 185.3 \quad 246.0$

$\begin{array}{lll}\text { n-butylcyclohexane } & 198.4 & 244.7\end{array}$

n-butylcyclopentane $\quad 165.2 \quad 238.4$

N-butyl-N'-(3,4-dichlorophenyl)-N-methylurea $\quad 374.3 \quad 413.4$

N-butyl-N-ethyl-2,6-dinitro-4-trifluoromethylaniline $\quad 338.5 \quad 278.6$

$\begin{array}{lll}\text { N-butylurea } & 369.3 & 371.6\end{array}$

n-decane $\quad 243.5 \quad 266.7$

N-decyl hexadecanamide $\quad 347.0 \quad 359.4$

n-decyl-a-cyanoacrylate $\quad 394.5 \quad 311.4$

$\begin{array}{lll}\text { n-decylcyclohexane } & 271.4 & 303.9\end{array}$

n-decylcyclopentane $\quad 251.0 \quad 294.4$

$\begin{array}{lll}\mathrm{N} \text {-decylundecanamide } & 344.0 & 351.6\end{array}$ 


\begin{tabular}{|c|c|c|}
\hline N-dimethylaminosuccinamic acid & 431.4 & 431.4 \\
\hline n-docosane & 316.1 & 316.2 \\
\hline n-dodecylcyclohexane & 258.8 & 313.5 \\
\hline n-dononacontahectane & 399.1 & 388.2 \\
\hline n-eicosane & 309.7 & 309.5 \\
\hline neopentyl-4,4,4-trinitrobutyrate & 333.5 & 361.4 \\
\hline n-ethyl-a-cyanoacrylate & 243.2 & 231.0 \\
\hline N-ethylurea & 365.1 & 364.8 \\
\hline n-hectane & 388.5 & 378.2 \\
\hline n-heneicosane & 313.7 & 313.0 \\
\hline n-heptacosane & 332.1 & 329.3 \\
\hline n-heptadecane & 295.1 & 297.1 \\
\hline N-heptylmyristamide & 343.0 & 351.6 \\
\hline n-hexacosane & 329.5 & 327.1 \\
\hline n-hexane & 177.8 & 212.3 \\
\hline N-hexyl decanamide & 311.0 & 340.0 \\
\hline N-hexyl hexadecanamide & 343.1 & 353.4 \\
\hline N-hexyl tetradecanamide & 334.0 & 349.6 \\
\hline nicosulfuron & 415.2 & 410.9 \\
\hline nicotinic acid & 510.0 & 400.8 \\
\hline $\mathrm{N}$-isopropylcarbazole & 395.2 & 348.6 \\
\hline $\mathrm{N}$-isopropylurea & 280.8 & 337.7 \\
\hline Nitralin & 425.2 & 432.0 \\
\hline nitrobenzene & 278.8 & 293.1 \\
\hline nitroethane & 183.7 & 174.4 \\
\hline nitromethane & 244.8 & 183.1 \\
\hline N-laurylnonanamide & 341.0 & 351.6 \\
\hline N-methyl O-methyl O-2-chloro-4-tert-butylphenyl phosphoramidate & 332.0 & 332.0 \\
\hline N-methyl-2,4,6,N-tetranitroaniline & 402.6 & 507.7 \\
\hline $\mathrm{N}$-methyl-2-chlorophenylcarbamic acid ester & 362.7 & 345.2 \\
\hline $\mathrm{N}$-methylacetamide & 303.8 & 300.6 \\
\hline N-methylcarbazole & 362.5 & 430.5 \\
\hline N-methyldiphenylacetamide & 439.8 & 413.4 \\
\hline N-methyl-N-nitrobutanamine & 331.0 & 283.8 \\
\hline N-methylpyrrole & 216.9 & 271.0 \\
\hline $\mathrm{N}$-methylurea & 378.1 & 360.1 \\
\hline N-myristylheptanamide & 334.0 & 351.6 \\
\hline $\mathrm{N}$-nitro-bis(N,N-cyanomethyl) amine & 367.0 & 378.1 \\
\hline N-nitro-N-methylaminomethane & 327.0 & 232.6 \\
\hline n-nonacosane & 336.6 & 333.5 \\
\hline n-octacosane & 334.5 & 331.5 \\
\hline n-octane & 216.4 & 244.1 \\
\hline n-octylbenzene & 234.2 & 281.7 \\
\hline nonadecane & 305.3 & 305.7 \\
\hline nonadecanoic acid & 341.2 & 347.3 \\
\hline nonanal & 253.9 & 276.5 \\
\hline nonane & 219.7 & 256.3 \\
\hline nonanoic acid & 285.5 & 321.1 \\
\hline nonyl acrylate & 236.5 & 303.8 \\
\hline nonyl phenylcarbamate & 327.0 & 362.8 \\
\hline norethindrone & 479.0 & 397.1 \\
\hline norethindrone acetate & 480.0 & 426.2 \\
\hline norgestrel & 482.2 & 456.1 \\
\hline
\end{tabular}




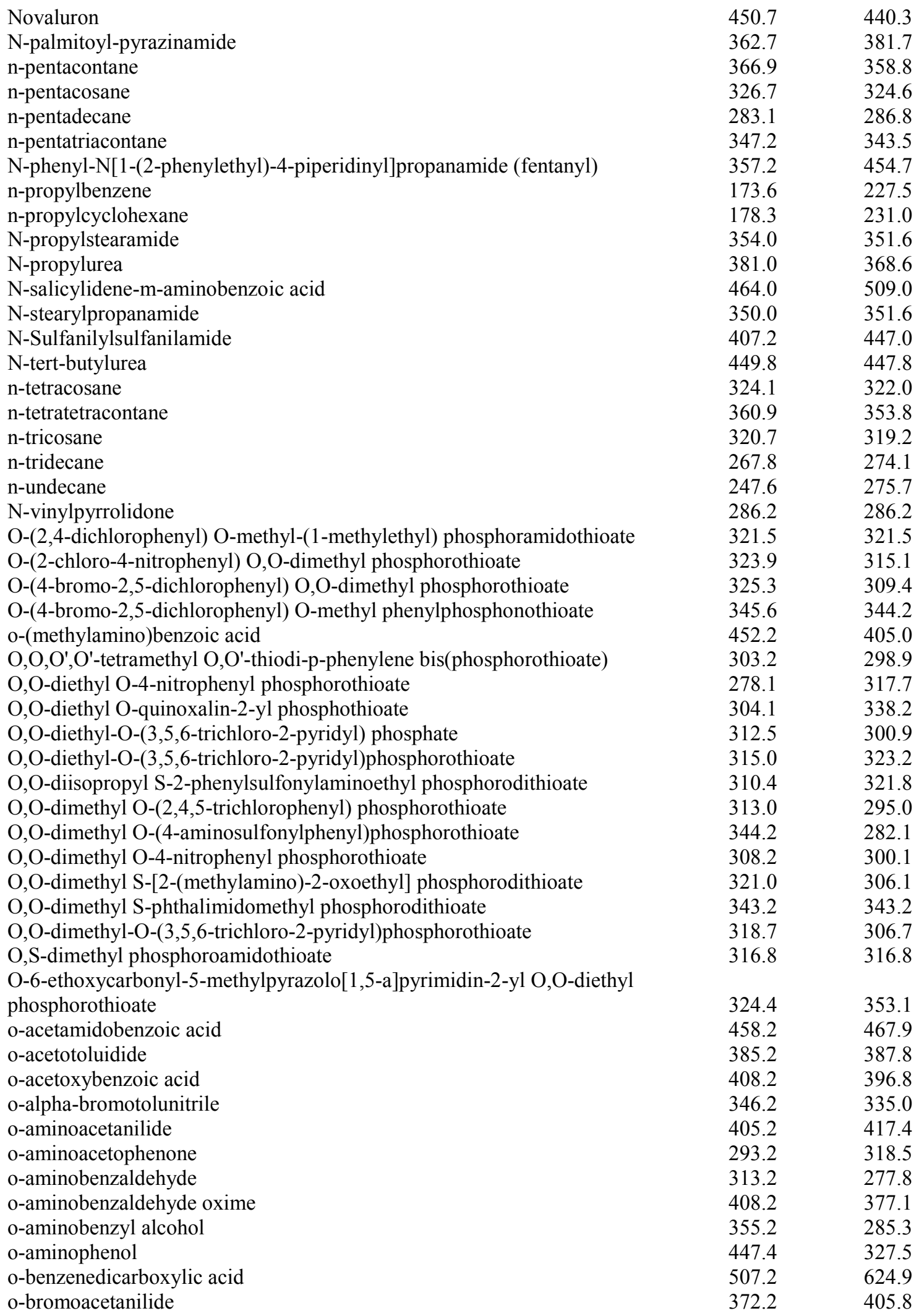




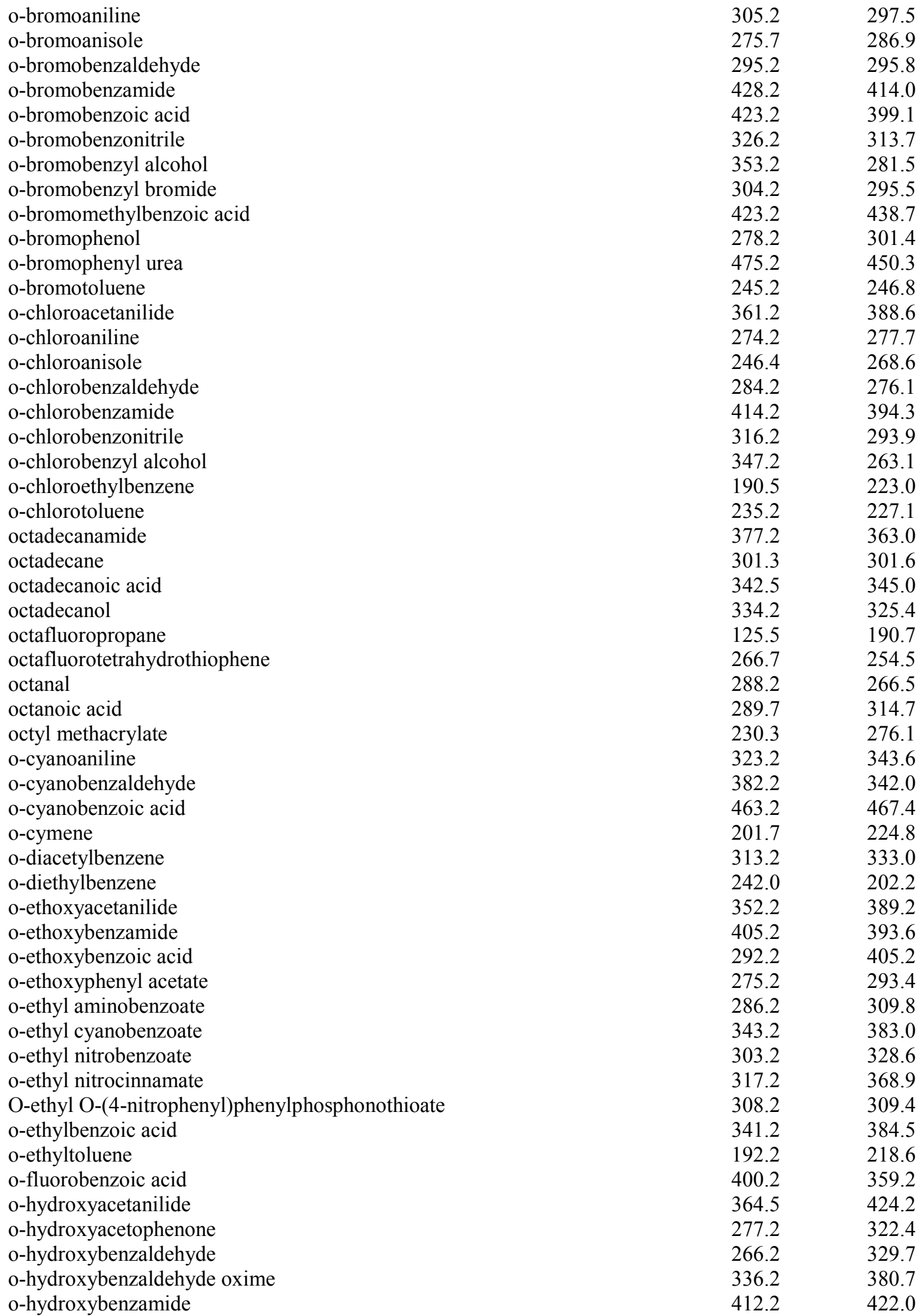




\begin{tabular}{|c|c|c|}
\hline o-hydroxybenzyl alcohol & 360.2 & 288.9 \\
\hline o-hydroxybiphenyl & 330.6 & 368.7 \\
\hline o-hydroxytoluene & 304.2 & 280.7 \\
\hline o-iodoacetanilide & 383.2 & 422.7 \\
\hline o-iodoaniline & 331.2 & 316.8 \\
\hline o-iodobenzaldehyde & 310.2 & 315.1 \\
\hline o-iodobenzaldehyde oxime & 381.2 & 367.2 \\
\hline o-iodobenzamide & 457.2 & 433.3 \\
\hline o-iodobenzyl alcohol & 363.2 & 299.5 \\
\hline o-iodobenzyl bromide & 330.2 & 313.5 \\
\hline o-iodophenol & 316.2 & 320.7 \\
\hline o-isopropylbenzoic acid & 337.2 & 390.8 \\
\hline o-methoxyacetanilide & 361.2 & 388.0 \\
\hline o-methoxyaniline & 279.2 & 314.8 \\
\hline o-methoxybenzaldehyde & 311.2 & 313.3 \\
\hline o-methoxybenzaldoxime & 365.2 & 383.5 \\
\hline o-methoxybenzamide & 402.2 & 392.8 \\
\hline o-methoxybenzoic acid & 374.2 & 406.0 \\
\hline o-methoxyphenol & 305.2 & 314.9 \\
\hline o-methoxyphenyl acetate & 283.2 & 281.3 \\
\hline o-methyl aminobenzoate & 297.2 & 298.3 \\
\hline o-methyl cyanobenzoate & 324.2 & 331.6 \\
\hline o-methyl hydroxybenzoate & 265.2 & 301.7 \\
\hline o-methyl nitrobenzoate & 260.2 & 319.5 \\
\hline o-methyl nitrocinnamate & 346.2 & 365.4 \\
\hline o-methylbenzyl alcohol & 309.2 & 282.8 \\
\hline o-N,N'-diacetylphenylenediamide & 459.2 & 420.2 \\
\hline o-nitroacetanilide & 367.2 & 411.3 \\
\hline o-nitroacetophenone & 301.2 & 342.8 \\
\hline o-nitrobenzaldehyde & 317.2 & 302.1 \\
\hline o-nitrobenzaldoxime & 376.2 & 375.6 \\
\hline o-nitrobenzamide & 448.2 & 455.4 \\
\hline o-nitrobenzoic acid & 419.0 & 440.6 \\
\hline o-nitrobenzonitrile & 383.2 & 342.0 \\
\hline o-nitrobenzoyl chloride & 293.2 & 319.4 \\
\hline o-nitrobenzyl alcohol & 347.2 & 320.1 \\
\hline o-nitrobenzyl bromide & 320.2 & 322.0 \\
\hline o-nitrobenzyl chloride & 322.2 & 306.7 \\
\hline o-nitrobenzyl cyanide & 357.2 & 348.4 \\
\hline o-nitrobenzyl iodide & 348.2 & 357.6 \\
\hline o-nitrobromobenzene & 315.2 & 299.7 \\
\hline o-nitroiodobenzene & 327.2 & 319.0 \\
\hline o-nitrophenentole & 275.3 & 323.8 \\
\hline o-nitrophenol & 318.2 & 307.7 \\
\hline o-nitrophenyl acetate & 314.2 & 310.1 \\
\hline o-nitrophenylacetic acid & 416.2 & 392.5 \\
\hline o-nitrotoluene & 269.2 & 275.2 \\
\hline o-N-methylhydroxyaniline & 370.2 & 341.0 \\
\hline o-N-methylnitroaniline & 310.2 & 318.9 \\
\hline o-N-methylphenylenediamine & 295.2 & 344.5 \\
\hline o-n-propylbenzoic acid & 331.2 & 386.3 \\
\hline o-phenylenediacetic acid & 423.2 & 418.5 \\
\hline o-phenylenediamine & 373.9 & 315.7 \\
\hline
\end{tabular}




\begin{tabular}{|c|c|c|}
\hline o-phenylenepyrene & 435.2 & 355.9 \\
\hline Orbencarb & 282.2 & 304.4 \\
\hline o-terphenyl & 329.4 & 371.0 \\
\hline o-tolualdehyde oxime & 322.2 & 326.4 \\
\hline o-toluamide & 413.2 & 415.4 \\
\hline o-toluic acid & 376.9 & 400.5 \\
\hline o-toluidine & 287.6 & 276.8 \\
\hline o-tolunitrile & 259.2 & 289.2 \\
\hline o-tolylurea & 464.2 & 443.5 \\
\hline Oxamyl & 374.2 & 373.2 \\
\hline oxandrolone & 498.2 & 432.8 \\
\hline Oxasulfuron & 431.2 & 491.9 \\
\hline o-xylene & 247.8 & 251.3 \\
\hline oxymetholone & 452.2 & 489.3 \\
\hline p-(methylamino)benzoic acid & 441.2 & 428.9 \\
\hline p,p'-dichlorobenzophenone & 420.0 & 375.3 \\
\hline p-acetamidobenzoic acid & 524.2 & 510.3 \\
\hline p-acetotoluidide & 427.2 & 387.8 \\
\hline p-a-cumylphenol & 346.4 & 320.3 \\
\hline p-alpha-bromotolunitrile & 389.2 & 335.0 \\
\hline p-aminoacetanilide & 436.2 & 435.3 \\
\hline p-aminobenzaldehyde & 345.2 & 372.4 \\
\hline p-aminobenzaldehyde oxime & 397.2 & 377.1 \\
\hline p-aminobenzamide & 387.2 & 469.9 \\
\hline p-aminobenzene sulphonamide & 439.3 & 305.1 \\
\hline p-aminobenzyl alcohol & 338.2 & 333.5 \\
\hline p-aminophenol & 462.5 & 378.7 \\
\hline p-benzoquinone & 388.0 & 421.0 \\
\hline p-bromoacetanilide & 440.2 & 409.2 \\
\hline p-bromoacetophenone & 324.2 & 314.5 \\
\hline p-bromoaniline & 339.2 & 340.4 \\
\hline p-bromoanisole & 286.2 & 286.9 \\
\hline p-bromobenzaldehyde & 340.2 & 338.6 \\
\hline p-bromobenzamide & 462.2 & 439.9 \\
\hline p-bromobenzoic acid & 525.2 & 480.2 \\
\hline p-bromobenzonitrile & 386.2 & 354.4 \\
\hline p-bromobenzyl alcohol & 350.2 & 305.6 \\
\hline p-bromobenzyl bromide & 336.2 & 295.5 \\
\hline p-bromoethynylbenzene & 338.2 & 320.7 \\
\hline p-bromoisopropylbenzene & 250.2 & 247.6 \\
\hline p-bromomethylbenzoic acid & 502.2 & 438.7 \\
\hline p-bromophenetole & 277.2 & 300.5 \\
\hline p-bromophenyl urea & 500.2 & 464.8 \\
\hline p-bromotoluene & 302.2 & 278.9 \\
\hline p-chloroacetanilide & 452.2 & 392.0 \\
\hline p-chloroacetophenone & 293.2 & 294.7 \\
\hline p-chloroaniline & 344.2 & 318.1 \\
\hline p-chlorobenzaldehyde & 320.2 & 316.2 \\
\hline p-chlorobenzamide & 452.2 & 420.2 \\
\hline p-chlorobenzonitrile & 369.2 & 332.1 \\
\hline p-chlorobenzyl alcohol & 348.2 & 287.2 \\
\hline p-chlorobenzyl bromide & 324.2 & 277.2 \\
\hline p-chlorobenzyl p-chlorophenyl sulfide & 343.8 & 346.1 \\
\hline
\end{tabular}




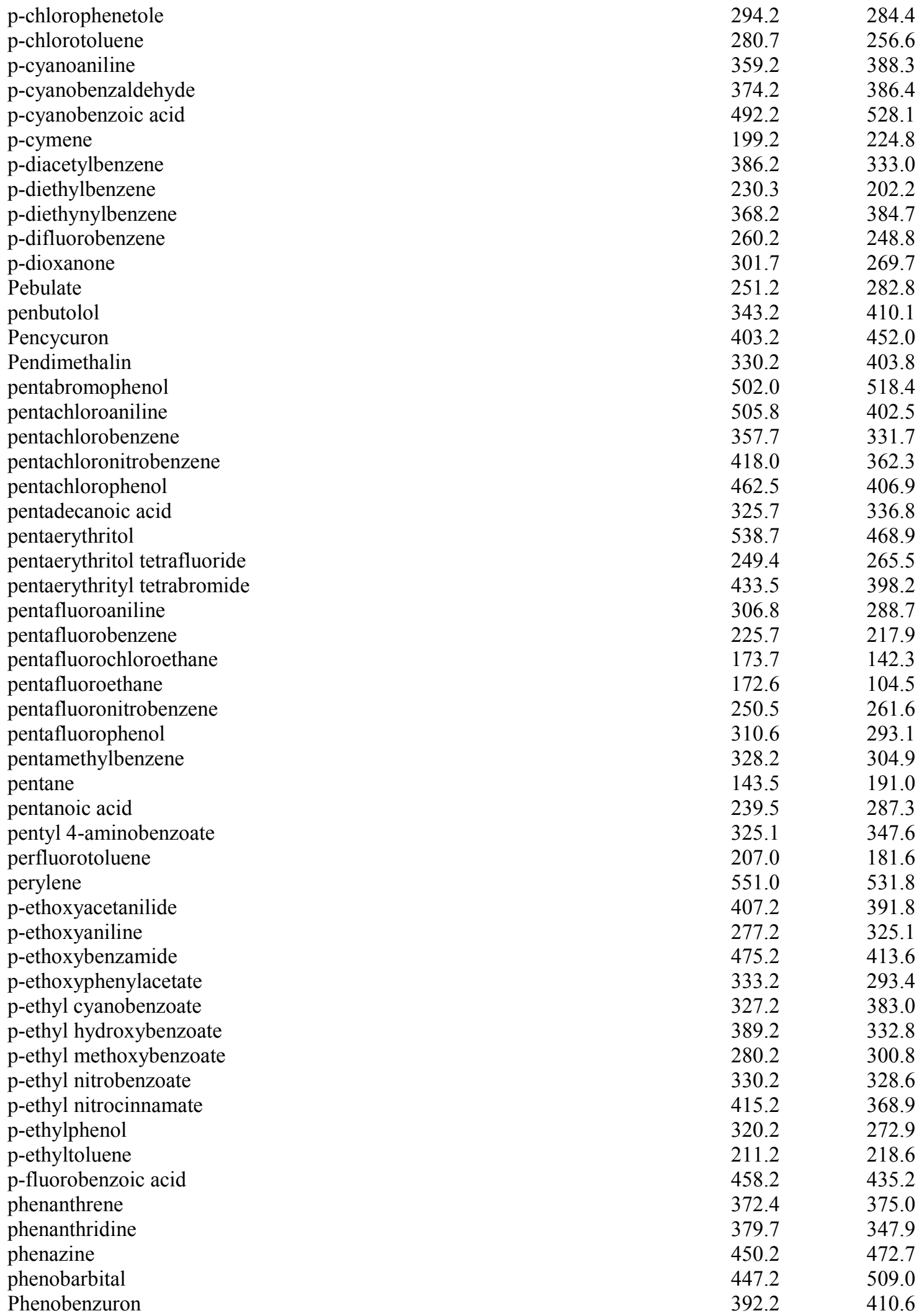




\begin{tabular}{|c|c|c|}
\hline phenol & 314.0 & 299.3 \\
\hline phenolphthalein & 534.0 & 624.3 \\
\hline phenoxathiin & 328.8 & 413.4 \\
\hline phenyl glycidyl ether & 279.8 & 326.4 \\
\hline phenyl vinyl sulfone & 343.4 & 305.3 \\
\hline phenylacetic acid & 349.9 & 332.6 \\
\hline phenylacetylene & 228.0 & 275.1 \\
\hline phenylaminoethyl methacrylate & 297.5 & 337.8 \\
\hline phenylhydrazine & 292.8 & 298.8 \\
\hline phenyl-o-tolylmethane & 279.8 & 297.2 \\
\hline phenylurea & 420.6 & 429.7 \\
\hline phthalazine & 364.5 & 287.1 \\
\hline phthalic anhydride & 403.3 & 375.7 \\
\hline p-hydroxyacetanilide & 441.2 & 438.7 \\
\hline p-hydroxyacetophenone & 382.2 & 348.3 \\
\hline p-hydroxybenzaldehyde & 389.2 & 376.8 \\
\hline p-hydroxybenzaldehyde oxime & 345.2 & 380.7 \\
\hline p-hydroxybenzamide & 435.2 & 473.8 \\
\hline p-hydroxybenzyl alcohol & 398.2 & 337.1 \\
\hline p-hydroxytoluene & 307.9 & 317.2 \\
\hline picene & 637.2 & 534.5 \\
\hline picolinic acid & 411.0 & 400.8 \\
\hline picric acid & 394.1 & 419.2 \\
\hline pimilic acid & 377.5 & 378.1 \\
\hline pindolol & 442.2 & 426.1 \\
\hline p-iodoacetanilide & 457.2 & 426.0 \\
\hline p-iodoacetophenone & 358.2 & 333.8 \\
\hline p-iodoaniline & 336.2 & 362.3 \\
\hline p-iodoanisole & 325.2 & 304.9 \\
\hline p-iodobenzaldehyde & 351.2 & 360.4 \\
\hline p-iodobenzamide & 491.2 & 459.2 \\
\hline p-iodobenzyl alcohol & 345.2 & 323.6 \\
\hline p-iodobenzyl bromide & 353.2 & 313.5 \\
\hline p-iodophenetole & 302.2 & 316.4 \\
\hline p-iodophenol & 367.2 & 366.7 \\
\hline p-iodophenyl acetate & 305.2 & 302.2 \\
\hline p-iodotoluene & 306.7 & 300.7 \\
\hline piperidine & 262.1 & 208.3 \\
\hline Pirimicarb & 363.7 & 311.2 \\
\hline p-isopropylbenzoic acid & 391.2 & 390.8 \\
\hline pivaldehyde & 272.1 & 225.1 \\
\hline p-methacryloyloxybenzoic acid & 455.0 & 398.0 \\
\hline p-methoxyacetanilide & 400.3 & 390.9 \\
\hline p-methoxyacetophenone & 312.2 & 306.1 \\
\hline p-methoxyaniline & 330.2 & 314.8 \\
\hline p-methoxybenzaldehyde & 275.2 & 313.3 \\
\hline p-methoxybenzaldoxime & 405.2 & 383.5 \\
\hline p-methoxybenzamide & 436.2 & 415.4 \\
\hline p-methoxyphenol & 329.2 & 318.5 \\
\hline p-methoxyphenyl acetate & 327.2 & 281.3 \\
\hline p-methyl bromobenzoate & 354.2 & 294.7 \\
\hline p-methyl chlorobenzoate & 317.2 & 277.5 \\
\hline p-methyl cyanobenzoate & 335.2 & 331.6 \\
\hline
\end{tabular}




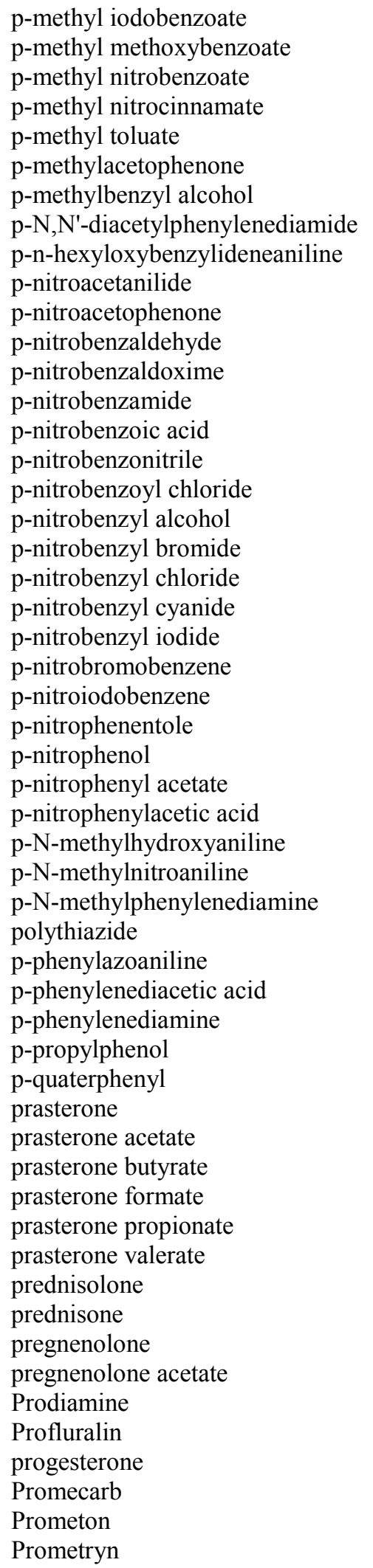

311.6

287.3

319.5

365.4

273.4

223.9

282.8

444.5

324.9

433.9

342.8

370.6

375.6

468.3

512.3

386.5

319.4

332.0

322.0

306.7

348.4

357.6

338.6

360.5

323.8

376.9

310.1

392.5

348.1

343.0

344.5

473.3

436.4

418.5

430.2

288.2

652.2

420.8

431.4

390.4

401.8

389.3

391.3

541.9

527.1

445.4

425.0

407.3

398.8

429.5

330.8

380.4

396.0 


\begin{tabular}{|c|c|c|}
\hline propanal & 171.3 & 175.1 \\
\hline propane & 85.5 & 129.4 \\
\hline Propazine & 486.2 & 377.3 \\
\hline propene & 88.2 & 77.5 \\
\hline propionic acid & 252.7 & 256.4 \\
\hline propionitrile & 180.4 & 199.0 \\
\hline Propoxur & 360.2 & 332.9 \\
\hline propranolol & 369.2 & 369.9 \\
\hline propyl 4-aminobenzoate & 347.1 & 336.9 \\
\hline propyl 4-hydroxybenzoate & 369.2 & 339.6 \\
\hline propyl N-phenyl carbamate & 331.0 & 342.3 \\
\hline propylcyclopentane & 155.8 & 222.8 \\
\hline propylene carbonate & 218.2 & 258.5 \\
\hline propylene oxide & 161.3 & 166.5 \\
\hline p-terphenyl & 486.3 & 517.3 \\
\hline p-tolualdehyde oxime & 353.2 & 326.4 \\
\hline p-toluamide & 432.2 & 415.4 \\
\hline p-toluic acid & 452.8 & 452.6 \\
\hline p-toluidine & 316.5 & 312.8 \\
\hline p-tolunitrile & 302.2 & 326.8 \\
\hline p-tolylurea & 456.2 & 443.5 \\
\hline p-xylene & 286.3 & 288.8 \\
\hline pyrazine & 328.2 & 289.3 \\
\hline pyrazole & 343.2 & 334.4 \\
\hline Pyrazosulfuron-ethyl & 454.7 & 453.7 \\
\hline pyrene & 423.8 & 440.1 \\
\hline pyridine & 231.5 & 233.6 \\
\hline pyromellitic dianhydride $1,2,5,6$-benzenetetracarboxylic dianhydride & 557.2 & 616.0 \\
\hline pyrrole & 249.7 & 330.7 \\
\hline pyrrolidine & 215.3 & 196.1 \\
\hline quinazoline & 320.9 & 293.4 \\
\hline quinoline & 258.4 & 277.3 \\
\hline quinoxaline & 305.7 & 331.5 \\
\hline Rimsulfuron & 450.2 & 455.4 \\
\hline S-(+)-4-isobutyl-a-methylphenyl acetic acid & 325.5 & 312.9 \\
\hline $\begin{array}{l}\text { S-(3,4-dihydro-4-oxobenzo[d]-[1,2,3]-triazin-3-ylmethyl) O,O-diethyl } \\
\text { phoshorodithioate }\end{array}$ & $322 ?$ & 338.2 \\
\hline S-(3,4-dihydro-4-oxobenzo[d][1,2,3]-triazin-3-ylmethyl) O,O-dimethyl & 32.2 & 350.2 \\
\hline phoshorodithioate & 345.3 & 326.3 \\
\hline S-2,3,3-trichloroallyl diisopropylthiocarbamate & 306.4 & 234.4 \\
\hline S-2,3-dihydro-5-methoxy-2-oxo-1,3,4-thiadiazol-3-ylmethyl-O,O-dimethyl & & \\
\hline phosphorodithioate & 315.1 & 315.1 \\
\hline sebacic acid & 404.0 & 382.4 \\
\hline Secbumeton & 361.2 & 338.3 \\
\hline sec-butylbenzene & 204.2 & 233.0 \\
\hline secobarbital & 373.2 & 387.7 \\
\hline siduron & 409.2 & 409.2 \\
\hline Simazine & 498.2 & 421.0 \\
\hline Simetryn & 356.2 & 365.5 \\
\hline spiropentane & 166.1 & 182.9 \\
\hline B,ß-binaphthyl & 461.2 & 492.3 \\
\hline ß-chloroacetic acid & 329.2 & 295.3 \\
\hline ß-chloroacetic acid & 329.2 & 295.3 \\
\hline
\end{tabular}


B-naphthol

ß-naphthyl acetate

ß-propiolactone

B-thiolactic acid

stanolone acetate

stanolone butyrate

styrene

suberic acid

succinic acid

sulfadiazine

sulfadicramide

sulfalene

sulfamerazine

sulfameter

sulfamethazine

sulfamethizole

sulfamethomidine

sulfametrole

sulfamoxole

Sulfanitran

Sulfaperine

Sulfaphenazole

Sulfapyrazine

Sulfapyridine

Sulfaquinoxaline

Sulfasomizole

Sulfasymazine

460.2

Sulfathiazole

475.2

Sulfazamet

468.2

Sulfisomidine

talbutal 
Terbumeton

396.7

410.3

Terbuthylazine

451.2

Terbutryn

378.2

terephthalyl dichloride

356.1

492.1

tert-butyl alcohol

299.0

424.4

tert-butyl amine

205.7

351.1

tert-butyl bromide

256.1

262.2

tert-butyl chloride

248.1

244.9

tert-butyl mercaptan

274.4

272.6

tert-butylbenzene

215.0

252.5

tert-butylmethylsulfone

357.6

203.5

testosterone

427.0

256.0

340.3

420.8

testosterone acetate

413.0

431.4

testosterone butyrate

382.0

390.4

testosterone formate

398.0

401.8

testosterone propionate

393.0

389.3

testosterone valerate

380.0

391.3

testosterone-17-beta-cypionate

374.2

411.0

tetra(methylthia)methane

338.7

244.9

tetrachloroethene

250.8

225.3

tetrachloro-o-xylene

359.2

304.7

tetrachloro-p-xylene

368.2

395.7

tetracontane

353.2

349.7

tetracyanoethylene

472.2

492.7

tetradecane

279.0

280.8

tetradecanoic acid

327.0

333.4

tetrafluoroethylene

142.0

120.7

tetrahydrofuran

164.8

210.6

tetramethylsuccinonitrile

442.0

350.1

tetramethylurea

272.2

227.2

tetramethysuccinic acid

464.0

428.0

tetratriacontane

345.9

342.0

tetrazole

432.1

theophylline

544.0

416.1

thiacyclobutane

199.9

579.3

thiacyclohexane

292.3

240.2

thiacyclopentane

177.0

255.6

248.8

thianthrene

429.6

514.3

Thiazafluron

410.2

372.4

thiazole

239.4

207.0

thidiazuron

486.2

488.2

Thiobencarb

276.5

304.4

Thiofanox

330.2

321.2

thiophene

235.2

215.8

thiophenol

258.2

258.2

thiourea

452.2

452.2

thioxanthene 50-195

401.8

422.6

thioxanthone

thymine

487.9

486.6

thymol

321.3

427.7

324.2

293.9

timolol

475.2

442.4

tolazamide

443.2

443.1

tolbutamide

401.2

366.4 


\begin{tabular}{|c|c|c|}
\hline toluene & 178.0 & 233.4 \\
\hline tolyl vinyl sulfone & 340.4 & 321.2 \\
\hline torsemide & 436.2 & 436.9 \\
\hline trans,cis-2,6-octadiene-1,8-dioic acid & 380.0 & 408.6 \\
\hline trans,trans-2,6-octadiene-1,8-dioic acid & 541.0 & 450.4 \\
\hline trans-1-(4-heptanoylphenyl)-4-heptylcyclohexane & 344.7 & 340.9 \\
\hline trans-1,2-cyclohexanediol & 372.3 & 269.8 \\
\hline trans-1,2-dimethylcyclohexane & 185.0 & 175.6 \\
\hline trans-1,3-dimethylcyclohexane & 183.1 & 175.6 \\
\hline trans-1,3-dimethylcyclopentane & 139.5 & 159.7 \\
\hline trans-1,3-pentadiene & 185.7 & 171.5 \\
\hline trans-1,4-dimethylcyclohexane & 236.2 & 175.6 \\
\hline trans-1,4-di-tert-butylcyclohexane & 363.2 & 277.9 \\
\hline trans-10-octadecenoic acid & 326.0 & 319.9 \\
\hline trans-11-octadecenoic acid & 317.0 & 319.9 \\
\hline trans-12-octadecenoic acid & 325.0 & 319.9 \\
\hline trans-13-octadecenoic acid & 318.0 & 319.9 \\
\hline trans-14-octadecenoic acid & 327.0 & 319.9 \\
\hline trans-15-octadecenoic acid & 331.0 & 319.9 \\
\hline trans-1-heptyl-4-(4-nonanoylphenyl)cyclohexane & 353.3 & 346.0 \\
\hline trans-2-butene & 167.6 & 116.2 \\
\hline trans-2-pentene & 133.0 & 100.4 \\
\hline trans-3-octadecenoic acid & 334.0 & 325.4 \\
\hline trans-4-octadecenoic acid & 333.0 & 319.9 \\
\hline trans-5-octadecenoic acid & 319.0 & 319.9 \\
\hline trans-6-octadecenoic acid & 326.0 & 319.9 \\
\hline trans-9-octadecenoic acid (elaidic acid) & 317.6 & 319.9 \\
\hline trans-azobenzene & 341.1 & 410.3 \\
\hline triacontane & 338.7 & 335.4 \\
\hline triamcinolone & 543.0 & 622.0 \\
\hline triamcinolone diacetate & 508.0 & 495.5 \\
\hline triamcinolone hexacetonide & 569.2 & 515.9 \\
\hline triasulfuron & 451.3 & 469.9 \\
\hline triatriacontane & 344.0 & 340.5 \\
\hline tribromomethane & 281.5 & 217.7 \\
\hline trichlormethiazide & 543.2 & 622.3 \\
\hline trichloroacetic acid & 330.7 & 345.1 \\
\hline trichloroethylene & 188.5 & 142.9 \\
\hline trichloromethane & 209.6 & 157.6 \\
\hline tridecandioic acid & 387.5 & 385.3 \\
\hline tridecanoic acid & 315.0 & 329.7 \\
\hline tridecanol & 306.6 & 305.7 \\
\hline Trietazine & 374.2 & 395.6 \\
\hline Triflumuron & 468.2 & 469.7 \\
\hline trifluoroacetonitrile & 128.7 & 195.1 \\
\hline trifluoromethane & 118.0 & 24.2 \\
\hline trifluoromethanethiol & 116.0 & 109.9 \\
\hline trifluoromethyl (2-hydroxy-1-propenyl)ketone & 232.4 & 293.1 \\
\hline Trifluralin & 321.7 & 365.1 \\
\hline trimellitic anhydride(1,2,4-benzenetricarboxylic acid) & 438.2 & 526.5 \\
\hline Trimethacarb & 383.2 & 366.0 \\
\hline trimethylamine & 156.1 & 130.7 \\
\hline trimethylhydrazine & 201.2 & 219.7 \\
\hline
\end{tabular}




\begin{tabular}{lcr} 
trinitroglycerine & 285.5 & 283.3 \\
triphenyl phosphate & 322.5 & 359.0 \\
triphenylamine & 400.2 & 358.3 \\
triphenylchloromethane & 376.8 & 441.7 \\
triphenylene & 471.0 & 572.7 \\
triphenylmethane & 365.3 & 343.1 \\
triphenylphosphine oxide & 431.9 & 431.9 \\
tri-tert-butylmethanol & 390.0 & 497.2 \\
undecanedioic acid & 385.0 & 383.5 \\
undecanoic acid & 301.6 & 331.4 \\
undecanolactone & 275.3 & 300.6 \\
urea & 408.1 & 408.1 \\
vinyl acetate & 180.6 & 137.8 \\
vinyl chloride & 119.3 & 82.2 \\
xanthene & 373.7 & 388.5 \\
xanthone & 449.7 & 452.6 \\
XMC & 372.2 & 353.7 \\
Xylylcarb & 352.7 & 353.7 \\
Z-3-chloro-2-butenoic acid & 366.8 & 347.2 \\
z-enantholactam & 310.3 & 286.0 \\
\hline
\end{tabular}




\section{REFERENCES}

(1) Abramowitz R, Yalkowsky SH. 1990. Melting Point, Boiling Point and Symmetry. Pharm. Res. 7: 942.

(2) Austin JB. 1930. A Relation between the Molecular Weight and Melting Points of Organic Compounds. J. Am. Chem. Soc. 52: 1049.

(3) Broadhurst MG. 1963. Thermodynamic Properties of Polyethylene predicted from paraffin data.J. Res. Natl. Bur. Std. A 67(3): 233.

(4) Budavari S. 1996. The Merck Index, Twelfth Edition. Merck \& Co. Inc., New Jersey.

(5) Charton M, Charton B. 1994. Quantitative Description of Structural Effects on Melting Points of Substituted Alkanes. J. Phys. Org. Chem. 7: 196.

(6) Chickos JS and Nichols G. 2002. The Estimation of Melting Points and Fusion Enthalpies Using Experimental Solubilities, Estimated Total Phase Change Entropies, and Mobile Order and Disorder Theory. J. Chem. Inf. Comp. Sci. 42: 368.

(7) Chickos JS, Acree WE Jr, and Liebman JF. 1999. Estimating Solid-Liquid Phase Change Enthalpies and Entropies. J. Phys. Chem. Ref. Data. 28 (6): 1535.

(8) Chickos JS, Hesse DG. 1990. Estimating Entropies and Enthalpies of Fusion of Hydrocarbons. J. Org. Chem. 55: 3833. 
(9) Chickos JS, Nichols G. 2001. Simple Relationships for the Estimation of Melting Temperatures of Homologous Series. J. Chem. Eng. Data. 46: 562.

(10) Constantinou L, Gani R. 1994. New Group Contribution Scheme for Estimating Properties of Pure Compounds. AIChE J. 40: 1697.

(11) Dannenfelser RM, Yalkowsky SH. 1996. Estimation of Entropy of Melting from Molecular Structure: A Non-Group Contribution Method. Ind. Eng. Chem. Res. 35: 1483 .

(12) Dannenfelser RM, Yalkowsky SH. 1999. Predicting the Total Entropy of Melting: Application to Pharmaceuticals and Environmentally Relevant Compounds. J. Pharm. Sci. 88 (7): 722.

(13) Dearden JC, Rahman MH. 1988. QSAR Approach to the Prediction of Melting Points of Substituted Anilines. Math. Comput. Model. 11: 843.

(14) Dearden JC. 1991. The QSAR prediction of melting point, a property of environmental relevance. Sci. Total Environ. 109/110: 59.

(15) Dearden JC. 2003. Quantitative Structure-Property Relationships for Prediction of Boiling point, Vapor Pressure and Melting point. Environ. Toxicol. Chem. 22(8): 1696.

(16) Dearden JC. 2003. Quantitative Structure-Property Relationships for Prediction of Boiling point, Vapor Pressure and Melting point. Environ. Toxicol. Chem. 22(8): 1696. 
(17) Flory PJ, Vrij A. 1963. Melting Points of Linear-Chain Homologs. The Normal Paraffin Hydrocarbons. J. Am. Chem. Soc 85: 3548.

(18) Gold PI, Ogle GJ. 1969. Estimating thermophysical properties of liquids. Part 4 Boiling, freezing and triple-point temperatures. Chem. Eng 76: 119.

(19) Hansch C and Leo A. 1979. Substituent Constants for Correlation Analysis in Chemistry and Biology, Wiley, New York.

(20) Howard PH and Meylan WM. 1997. Handbook of Physical Properties of Organic Chemicals. CRC Press Inc., Florida.

(21) http:// chemfinder.cambridgesoft.com 2004. CambridgeSoft Corporation.

(22) Jain A, Yang G, and Yalkowsky SH. 2004. Estimation of Melting Points of Organic Compounds. Ind. Eng. Chem. Res. 43 (23): 7618.

(23) Jain A, Yang G, and Yalkowsky SH. 2004. Estimation of Total Entropy of Melting of Organic Compounds. Ind. Eng. Chem. Res. 43 (15): 4376.

(24) Jain N, Yalkowsky SH. 1999. UPPER III: Unified Physical Property Estimation Relationships: Application to Non-Hydrogen Bonding Aromatic Compounds. J. Pharm. Sci. 88: 852.

(25) Jain N, Yalkowsky SH. 2001. Estimation of the aqueous solubility I: Application to organic non-electrolytes. J. Pharm. Sci. 90: 234.

(26) Joback KG, Reid RC. 1987. Estimation of Pure Component Properties from Group Contributions. Chem. Eng. Commun. 57: 233. 
(27) Joback KG. 1984. A Unified Approach to Physical Property Estimation Using Multivariate Statistical Techniques. Stevens Institute of Technology, submitted to the Dept. of Chem. Eng. for M.S. Degree at the Massachusetts Institute of Technology in June 1984.

(28) Katritzky AR, Gordeeva EV. 1993. Traditional Topological Indices vs Electronic, Geometrical, and Combined Molecular Descriptors in QSAR/QSPR Research. J. Chem. Inf. Comput. Sci. 33: 835.

(29) Katritzky AR, Jain R, Lomaka A, Petrukhin R, Maran, U, Karelson M. 2001. Perspective on the Relationship between Melting Points and Chemical Structure. Crystal Growth and Design. 1 (4): 261.

(30) Katritzky AR, Maran U, Karelson M, and Lobanov VS. 1997. Prediction of Melting Points for the Substituted Benzenes: A QSPR Approach. J. Phys. Chem. Ref. Data. 37: 913.

(31) Kier LB, Hall LH. 1976. Molecular Connectivity in Chemistry and Drug Research. Academic Press: New York.

(32) Kirshenbaum I. 1965. Entropy and Heat of Fusion of Polymers. J. Polymer. Sci. A 3: 1869.

(33) Krzyzaniak JF, Myrdal PB, Simamora P, Yalkowsky SH. 1995. Boiling Point and Melting Point Prediction for Aliphatic, Non-hydrogen Bonding Compounds. Ind. Eng. Chem. Res. 34: 2530. 
(34) Law D, Wang W, Schmitt EA, Long MA. 2002. Prediction of poly-(ethylene) glycol-drug eutectic compositions using an index based on the van't Hoff equation. Pharm. Res. 19: 315.

(35) Leo A. 1993. Calculating logPoct from Structures. Chemical Reviews. 93: 1281.

(36) Lyman WJ. 1985. In: Environmental Exposure from Chemicals. Volume I., Neely WB and Blau GE (editors), Boca Raton, FL: CRC Press, Inc., Chapter 2.

(37) Marrero J, Gani R. 2001. Group-Contribution based estimation of pure component properties. Fluid Phase Equil. 183: 183.

(38) MPBPWIN V1.41. 2000. U.S. Environmental Protection Agency.

(39) Needham DE, Wei I, Seybold PG. 1988. Molecular Modelling of the Physical Properties of the Alkanes. J. Am. Chem. Soc. 110: 4186.

(40) Nikmo J, Kukkonen J, Riikonen K. 2002. A model for evaluating physicochemical substance properties required by consequence analysis models. J. Hazard. Mater. 91: 43.

(41) Ran Y, Yalkowsky SH. 2001. Prediction of drug solubility by the general solubility equation (GSE). J. Chem. Inf. Comp. Sci. 41: 354.

(42) Reid RC, Prauznitz JM, Poling BE. 1987. The Properties of Gases and Liquids. Fourth Edition. New York: McGraw-Hill, Inc., Chapter 2. 
(43) Simmamora P and Yalkowsky SH. 1994. Group Contribution Methods for Predicting the Melting Point and Boiling Point of Aromatic Compounds. Ind. Eng. Chem. Res. 33: 1405.

(44) Starkweather HW Jr, Boyd RH. 1960. The Entropy and Melting of Some Linear Polymers. J. Phys. Chem 64: 410.

(45) Tonelli AE. 1970. Calculation of the Intramolecular Contribution to the Entropy of Fusion in Crystalline Polymers. J. Chem. Phys. 52 (9): 4749.

(46) Tsakanikas PD, Yalkowsky SH. 1988. Estimation of Melting Point of Flexible Molecules: Aliphatic Hydrocarbons. Toxic. Environ. Chem. 17: 19.

(47) Yalkowsky SH, Dannenfelser RM, Myrdal PB, Simamora P, and Mishra DS. 1994. Unified Physical Property Estimation Relationships (UPPER). Chemosphere. 28: 1657.

(48) Yalkowsky SH, Myrdal PB, Dannenfelser RM, and Simamora P. 1994. UPPER II: Calculation of Physical Properties of the Chlorobenzenes. Chemosphere. 28: 1675.

(49) Yalkowsky SH. 1979. Estimation of Entropies of Fusion of Organic Compounds. I \& EC Fundamentals. 18: 108.

(50) Yalkowsky SH. 1981. Solubility and partitioning V: Dependence of solubility on melting point. J. Pharm. Sci. 70: 971. 